\title{
Oxygen Donor-Mediated Equilibration of Diastereomeric Alkene-Palladium(II) Intermediates in Enantioselective Desymmetrizing Heck Cyclizations
}

\author{
Axel B. Machotta, ${ }^{\dagger}$ Bernd F. Straub, ${ }^{,{ }^{\star}}$ and Martin Oestreich ${ }^{\star,}{ }^{\dagger}$ \\ ${ }^{\dagger}$ Organisch-Chemisches Institut, Westfälische Wilhelms-Universität Münster, \\ Corrensstrasse 40, D-48149 Münster, Germany \\ martin.oestreich@uni-muenster.de \\ ${ }^{\ddagger}$ Organisch-Chemisches Institut, Ruprecht-Karls-Universität Heidelberg, \\ Im Neuenheimer Feld 270, D-69120 Heidelberg, Germany \\ straub@oci.uni-heidelberg.de
}

\section{Supporting Information}

\section{Contents}

Starting Material Syntheses 


\section{$1 \quad$ Starting Material Syntheses}

All cyclization precursors contain a stereodefined 1,7-diphenyl-1,6-heptadienyl unit. In the light of the strong influence of the double bond geometry on the stereochemical outcome of asymmetric Heck processes, ${ }^{1}$ highly stereoselective methods were required for their preparation. At the outset of the project, we first targeted the synthesis of $(E, E)-7(X=B r)$ and $(E, E)-9(X=O T f)$ as these resemble the general mandatory structural motif 5 (Scheme 2). We learned though that the obvious route to access these by direct two-fold allylation of the corresponding unactivated esters is rather delicate. It remains an open challenge to control both regioselectivity ( $\alpha$-versus $\gamma$-attack) and diastereoselectivity ( $E$ versus $Z$ ) when using $\gamma$-substituted allyl metals. ${ }^{2}$ In order to avoid this dilemma, we elaborated a stereodivergent procedure for the regio- and diastereocontrolled preparation of substituted bishomoallylic alcohols by dividing these issues into two separate synthetic operations, $\alpha$ regioselective bispropargylation using propargylic zinc reagent $\mathbf{S I} 4$ followed by $E$ or Z-selective reduction of the carbon-carbon triple bond. ${ }^{3}$

Thus, SI4 generated from 3-bromo-1-trimethylsilyl-1-propyne and activated zinc was reacted with enolizable lactone $\mathbf{S I} 2$ and ester SI3 at room temperature. This straightforward procedure allowed for the regioselective two-fold propargylation in excellent yields (SI2-SI3 $\rightarrow$ SI6-SI7, Scheme SI1). In order to install the aryl groups, alkynes SI6-SI7 were deprotected using substoichiometric amounts of TBAF $3 \mathrm{H}_{2} \mathrm{O}$ followed by conventional Sonogashira reaction in high overall yields $(\mathbf{S I 6}-\mathrm{SI7} \rightarrow \mathrm{SI9}-\mathrm{SI} 10 \rightarrow \mathrm{SI12}-\mathrm{SI} 13$, Scheme SI1). An attempt to directly use SI6 in the cross-coupling failed. ${ }^{4}$ The diasteroselectivity-determining reduction required careful use of aluminum-based reducing agents. Whereas clean reduction of SI12 was observed using $\mathrm{LiAlH}_{4}(\mathrm{SI12} \rightarrow(E, E)-\mathrm{SI} 15, \mathrm{Scheme}$ SI1), the related reduction of SI13 produced an intractable mixture of products. We soon realized that treatment of $\mathrm{SI} 13$ with $\mathrm{LiALH}_{4}$ or Red-Al ${ }^{\circledR}$ was not chemoselective and was accompanied by substantial dehalogenation. ${ }^{5}$ To our delight, excess of DIBAL-H at elevated temperatures resulted in a flawless reduction $(\mathbf{S I} 13 \rightarrow(E, E)-7$, Scheme SI1). In both cases, isolated yields and diastereoselectivities $(E: Z \geq 99: 1)$ were excellent. Selective triflation of the phenol in the presence of the tertiary alcohol proceeded smoothly $((E, E)-$ SI15 $\rightarrow(E, E)-9)$.

\footnotetext{
${ }^{1}$ Ashimori, A.; Matsuura, T.; Overman, L. E.; Poon, D. J. J. Org. Chem. 1993, 58, 6949-6951.

${ }^{2}$ Yanagisawa, A.; Yamamoto, H. In Advances in Carbon Chemistry; Snieckus V., Ed.; JAI Press: London, 1996; Vol. 2, pp. 87-110.

${ }^{3}$ Oestreich, M.; Sempere-Culler, F. Chem. Commun. 2004, 692-693.

${ }^{4}$ Yang, C.; Nolan, S. P. Organometallics 2002, 21, 1020-1022.

${ }^{5}$ Beckwith, A. L. J.; Goh, S. H. Chem. Commun. 1983, 905-906.
} 
Scheme SI1. Preparation of Cyclization Precursors $(E, E)-7-(E, E)-11^{a}$

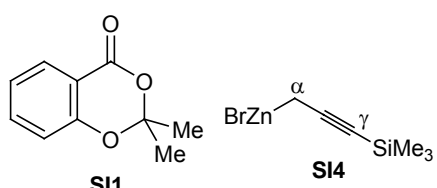

SI1<smiles>O=C1Cc2ccccc2O1</smiles>

SI2<smiles>CCOC(=O)Cc1ccccc1Br</smiles>

SI3

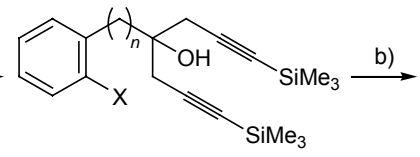

$\mathrm{SI5}(\mathrm{X}=\mathrm{OH}, n=0): 92 \%$ SI6 (X $=\mathrm{OH}, n=1): 86 \%$ $\operatorname{SI7}(\mathrm{X}=\mathrm{Br}, n=1): 84 \%$ $\alpha: \gamma \geq 98: 2$

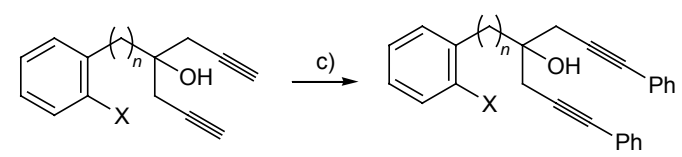

SI8 $(\mathrm{X}=\mathrm{OH}, n=0): 78 \%$ SI9 (X=OH, $n=1): 85 \%$ SI10 (X = Br, $n=1): 90 \%$

SI11 (X $=\mathrm{OH}, n=0): 91 \%$ SI12 (X=OH, $n=1): 94 \%$ $\operatorname{SI13}(\mathrm{X}=\mathrm{Br}, n=1): 96 \%$

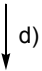

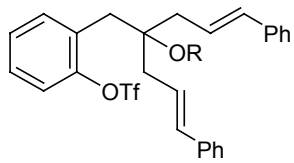

$(E, E)-10\left(\mathrm{R}=\mathrm{SiEt}_{3}\right): 51 \%$ $(E, E)-11(\mathrm{R}=\mathrm{Me}): 49 \%$

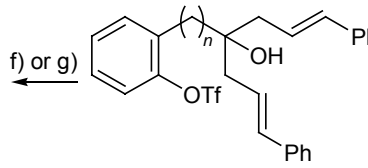

$(E, E)-8(n=0): 87 \%$ $(E, E)-9(n=1): 94 \%$

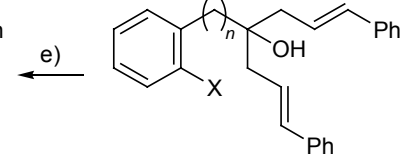

$(E, E)$-SI14 $(X=\mathrm{OH}, n=0): 91 \%$ $(E, E)$-SI15 (X = OH, $n=1): 94 \%$ $(E, E)-7(\mathrm{X}=\mathrm{Br}, n=1): 95 \%$ $E: Z \geq 99: 1$

${ }^{a}$ Reaction conditions: a) SI4, THF, RT; b) TBAF $3 \mathrm{H}_{2} \mathrm{O}$, THF, RT; c) Phl, $\left(\mathrm{Ph}_{3} \mathrm{P}\right)_{2} \mathrm{PdCl}_{2}$, Cul, Et ${ }_{3} \mathrm{~N}, \mathrm{RT}$ d) for SI11: Red-Al ${ }^{\circledR}$, THF, $0^{\circ} \mathrm{C} \rightarrow \mathrm{RT}$; for SI12: $\mathrm{LiAlH}_{4}$, THF, $0^{\circ} \mathrm{C} \rightarrow \mathrm{RT}$; for SI13: DIBAL-H, THF, 65 ${ }^{\circ} \mathrm{C}$; e) for $(E, E)-\mathrm{SII4}_{2} \mathrm{Tf}_{2} \mathrm{O}$, 2,6-lutidine, DMAP, $\mathrm{CH}_{2} \mathrm{Cl}_{2},-30^{\circ} \mathrm{C} \rightarrow \mathrm{RT}$; for (E,E)-SI15: $\mathrm{PhNTf}_{2}, \mathrm{Cs}_{2} \mathrm{CO}_{3}$, DMF, RT; f) $\mathrm{Et}_{3} \mathrm{SiOTf}, 2$,6-lutidine, $\left.\mathrm{CH}_{2} \mathrm{Cl}_{2}, 0^{\circ} \mathrm{C} \rightarrow \mathrm{RT} ; \mathrm{g}\right) \mathrm{Me}_{3} \mathrm{O}^{+} \mathrm{BF}_{4}^{-}$, Proton-sponge ${ }^{\mathrm{TM}}, \mathrm{CH}_{2} \mathrm{Cl}_{2}$, RT. DIBAL-H=diisobutylaluminum hydride, DMAP=4$\mathrm{N}, \mathrm{N}$-dimethylaminopyridine, $\mathrm{DMF}=\mathrm{N}, \mathrm{N}$-dimethylaminoformamide, Proton-sponge ${ }^{\mathrm{TM}}=1,8$-bis(dimethylamino)naphthalene, Red-A ${ }^{\circledR}=$ sodium bis(2-methoxyethoxy)aluminum dihydride, TBAF=tetra- $n$-butylammonium fluoride, $\mathrm{Tf}=$ =trifluoromethanesulfonyl.

The same sequence was employed for the synthesis of the corresponding cyclization precursor $(E, E)$ 8 (Scheme SI1). Starting from acetonide SI1, chemical yields and selectivities were comparable to those of the previous syntheses; Red-Al ${ }^{\circledR}$ was superior to $\mathrm{LiAlH}_{4}$ in the reduction step $(\mathrm{SII1} \rightarrow(E, E)$ SI14) and the subsequent triflation was rather capricious $((E, E)-S \mid 14 \rightarrow(E, E)-8),{ }^{6}$ which is probably due to the close proximity of the hydroxy groups.

Evidence for a directing effect of the hydroxy group would also be derived from Heck ring closures of cyclization precursors incorporating cognate donors having modified steric and electronic properties. We therefore prepared the requisite silyl ether $(E, E)-\mathbf{1 0}$ and methyl ether $(E, E)-\mathbf{1 1}$ (Scheme SI1); their syntheses were severely complicated by migration processes of the triflyl group. We finally succeeded using $\mathrm{Et}_{3}$ SiOTf in the silylation $((E, E)-\mathbf{9} \rightarrow(E, E)-10)^{7}$ and Meerwein's salt in the methylation $((E, E)$ $\mathbf{9} \rightarrow(E, E)-\mathbf{1 1}) .{ }^{8}$ As expected, yields were generally poor yet unreacted $(E, E)-\mathbf{9}$ was quantitatively reisolated in the latter case.

As deoxygenation of any of the tertiary alcohol-containing substrates (Scheme SI1) proved fruitless, we chose a different approach for the diastereoselective synthesis of the deoxygenated cyclization precursor $(E, E)$-12 (Scheme SI2). A procedure described by Tamaru perfectly matched our purposes; ${ }^{9}$

\footnotetext{
${ }^{6}$ Dolle, R. E.; Schmidt, S. J.; Kruse, L. I. J. Chem. Soc., Chem. Commun. 1987, 904-905.

${ }^{7}$ Guanti, G.; Banfi, L.; Riva, R. Tetrahedron 1994, 50, 11945-11966.

${ }^{8}$ Evans, D. A.; Ratz, A. M.; Huff, B. E.; Sheppard, G. S. Tetrahedron Lett. 1994, 35, 7171-7172.

${ }^{9}$ Horino, Y.; Naito, M.; Kimura, M.; Tanaka, S.; Tamaru, Y. Tetrahedron Lett. 2001, 42, 3113-3116.
} 
acetophenones having an ortho hydroxy group undergo regio- and stereoselective palladiumcatalyzed and borane-mediated allylation with allylic alcohols. According to the reported protocol, compound SI16 was transformed into $(E, E)$-SI17 in useful yield (Scheme SI2). This facile construction of the 1,7-diphenyl-1,6-heptadienyl unit left us with a particularly demanding deoxygenation $((E, E)$ SI17 $\rightarrow(E, E)$-SI19). After standard single-step protocols failed to produce any $(E, E)$-SI19, we applied a two-step sequence previously used by Angle. ${ }^{10}$ Carbonyl reduction of $(E, E)$-SI17 afforded $(E, E)$-SI18, which was then permesylated followed by treatment with $\mathrm{LiAlH}_{4}$ and aqueous $\mathrm{NaOH}$ in hot $\mathrm{MeOH}$ $((E, E)$-SI18 $\rightarrow(E, E)$-SI19). Triflation under standard conditions completed the synthesis of the deoxygenated cyclization precursor $((E, E)-$ SI19 $\rightarrow(E, E)-12)$, Scheme SI2).

Scheme S12. Preparation of Deoxygenated Cyclization Precursor $(E, E)-\mathbf{1 2}^{a}$

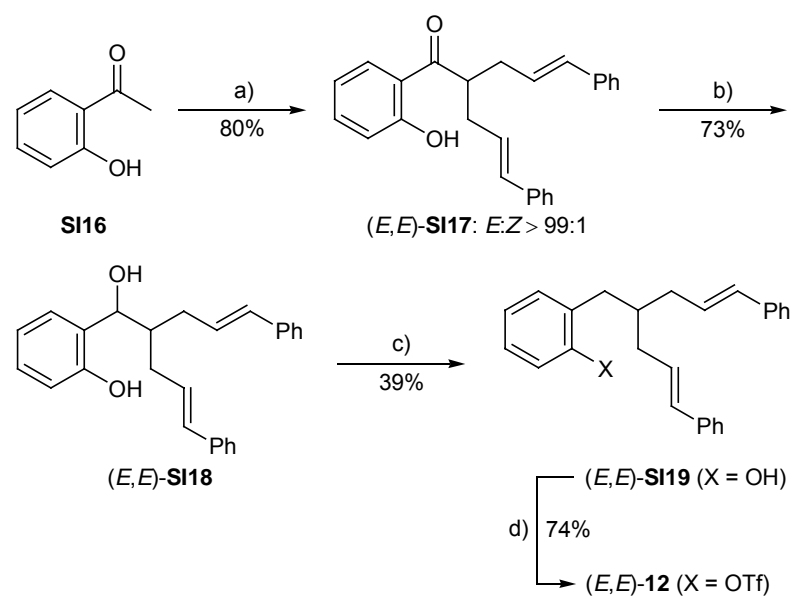

${ }^{a}$ Reaction conditions: a) cinnamic alcohol, $\mathrm{Pd}(\mathrm{OAc})_{2}, \mathrm{Ph}_{3} \mathrm{P}, \mathrm{Et}_{3} \mathrm{~B}, \mathrm{THF}, 50^{\circ} \mathrm{C}$; b) $\left.\left.\mathrm{LiAlH}_{4}, \mathrm{THF}, 0^{\circ} \mathrm{C} \rightarrow \mathrm{RT} ; \mathrm{c}\right) \mathrm{i}\right) \mathrm{MsCl}$, $\mathrm{Et}_{3} \mathrm{~N}, \mathrm{Et}_{2} \mathrm{O}, 0^{\circ} \mathrm{C}$; ii) $\mathrm{LiAlH}_{4}, 0^{\circ} \mathrm{C}$; iii) aq $\mathrm{NaOH}, \mathrm{MeOH}, 50^{\circ} \mathrm{C}$; d) $\mathrm{PhNTf}_{2}, \mathrm{Cs}_{2} \mathrm{CO}_{3}, \mathrm{DMF}, \mathrm{RT}$. Ms=methanesulfonyl.

The desymmetrizing Heck reaction of acyclic cyclization precursors also allows to test the influence of the double bond geometry on enantioselectivity. The requisite substrates are accessible by the strategy outlined above; two-fold propargylation is followed by Z- (Scheme SI3) instead of E-selective (Scheme SI1) alkyne reduction. Lindlar hydrogenation of SI12 was, however, either incomplete using $\mathrm{Pd} / \mathrm{CaCO}_{3}$ or over-reduction occurred using $\mathrm{Pd} / \mathrm{BaSO}_{4}$. A reversed order, triflation first $(\mathbf{S I} \mathbf{1 2} \rightarrow \mathbf{S I 2 0})$ and then Lindlar hydrogenation using $\mathrm{Pd} / \mathrm{CaCO}_{3}(\mathrm{SI20} \rightarrow(Z, Z)-9)$, was successful (Scheme SI3). The methyl ether $(Z, Z)-\mathbf{1 1}$ was again accessed by reaction with Meerwein's salt $((Z, Z)-\mathbf{9} \rightarrow(Z, Z)-\mathbf{1 1})$.

Scheme SI3. Preparation of Cyclization Precursors $(Z, Z)-\mathbf{9}$ and $(Z, Z)-11^{a}$

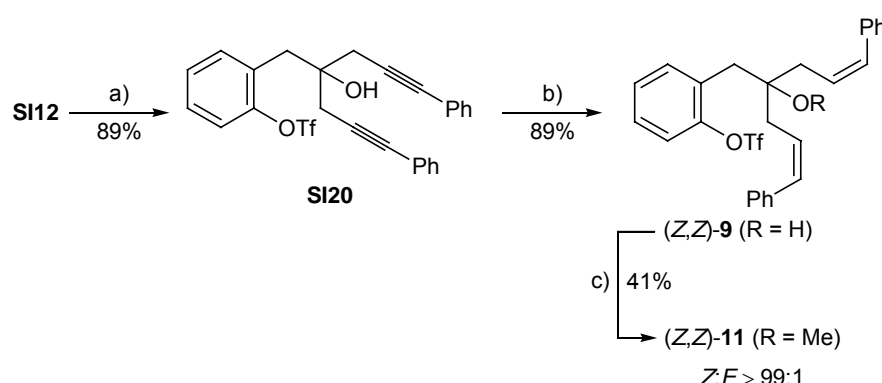

${ }^{a}$ Reaction conditions: a) $\mathrm{PhNTf}_{2}, \mathrm{Cs}_{2} \mathrm{CO}_{3}, \mathrm{DMF}, \mathrm{RT}$; b) $\mathrm{H}_{2}, \mathrm{Pd} / \mathrm{CaCO}_{3}$, quinoline, EtOH, RT; c) $\mathrm{Me}_{3} \mathrm{O}^{+} \mathrm{BF}_{4}^{-}$, Proton-sponge ${ }^{\mathrm{TM}}, \mathrm{CH}_{2} \mathrm{Cl}_{2}$, RT.

${ }^{10}$ Angle, S. R.; Frutos, R. P. J. Org. Chem. 1993, 58, 5135-5144. 


\section{General Information}

General Remarks: Reagents obtained from commercial suppliers were used without further purification unless otherwise noted. All reactions were performed in flame-dried glassware under a static pressure of argon. Liquids and solutions were transferred with syringes or double-ended needles. Solvents were dried prior to use following standard procedures ( $\mathrm{THF}, \mathrm{CH}_{2} \mathrm{Cl}_{2}$, toluene) and technical grade solvents for extraction or chromatography (cyclohexane, $t$-butyl methyl ether) were distilled before use. Analytical thin-layer chromatography was performed on silica gel SIL G-25 glass plates by Macherey-Nagel/Germany and flash chromatography on silica gel 60 (40-63 $\mu \mathrm{m}, 230-400$ mesh, ASTM) by Merck/Germany using the indicated solvents. ${ }^{1} \mathrm{H}$ and ${ }^{13} \mathrm{C}$ NMR spectra were recorded in $\mathrm{CDCl}_{3}$ on Bruker AM 400 and DRX 500 instruments. Analytical HPLC analysis on a chiral stationary phase using Daicel Chiralcel $\mathrm{AD}$ and $\mathrm{AD}-\mathrm{H}$ columns ( $n$-heptane/i-PrOH mixtures as solvent) provided baseline separation of enantiomers.

\section{$3 \quad$ Typical Procedures Including Characterization Data}

\section{2-[2-Hydroxy-5-trimethylsilyl-2-(3-trimethylsilyl-2-propynyl)-4-propynyl]phenol (SI6)-typical} procedure for the two-fold bispropargylation of non-activated esters: A two-necked flask equipped with a reflux condenser and a magnetic stirring bar was charged with zinc dust $(5.88 \mathrm{~g}, 90.0$ $\mathrm{mmol}$ ) and flame-dried in vacuo until sublimation of zinc occurred. The thermally activated zinc metal was allowed to cool to room temperature before backfilling with argon and suspending in dry THF (100 $\mathrm{mL})$. In three portions, 1,2-dibromoethane $(0.90 \mathrm{ml}$ overall) was added followed by heating to reflux. After adding $\mathrm{Me}_{3} \mathrm{SiCl}(0.45 \mathrm{~mL})$ in one portion, the reaction mixture was maintained at ambient temperature for $30 \mathrm{~min}$. Dropwise addition of 3-bromo-1-trimethylsilyl-1-propyne $(8.60 \mathrm{~g}, 45.0 \mathrm{mmol})$ resulted in an exothermic reaction and formation of propargylic zinc reagent SI4 was completed at room temperature overnight. A solution of 3H-benzo-2-furanone (SI2) $(2.01 \mathrm{~g}, 15.0 \mathrm{mmol})$ and dry THF (10 mL) was added via syringe and the resulting mixture was maintained for two days at ambient temperature. The reaction was quenched with aq $\mathrm{HCl}(2 \mathrm{M}, 120 \mathrm{~mL})$ under vigorous stirring and filtered. The filtrate was diluted with $t$-butyl methyl ether $(250 \mathrm{~mL})$, the aqueous phase extracted with $t$-butyl methyl ether $(100 \mathrm{~mL})$, and the combined organic phases were washed with saturated aq $\mathrm{NaHCO}_{3}$ $(100 \mathrm{~mL})$ and dried $\left(\mathrm{MgSO}_{4}\right)$. The solvents were evaporated under reduced pressure and the residue was purified by flash column chromatography on silica gel (cyclohexane/t-butyl methyl ether 9:1) affording $\operatorname{SI6}\left(4.60 \mathrm{~g}, 86 \%\right.$ ) as a colorless solid. $R_{\mathrm{f}}=0.36$ (cyclohexane/t-butyl methyl ether 6:1); m.p. 85-86 ${ }^{\circ}$; ${ }^{1} \mathrm{H}$ NMR $\left(400 \mathrm{MHz}, \mathrm{CDCl}_{3}\right): \delta=7.91$ (br s, $\left.1 \mathrm{H}\right), 7.16$ (dd, J=7.7, 7.7 Hz, $1 \mathrm{H}$ ), 7.07 (d, J=7.7 $\mathrm{Hz}, 1 \mathrm{H}$ ), 6.92 (d, J=8.2 Hz, $1 \mathrm{H}$ ), 6.83 (dd, J=7.5, $7.5 \mathrm{~Hz}, 1 \mathrm{H}$ ), 3.14 (br s, $1 \mathrm{H}$ ), 3.01 (s, $2 \mathrm{H}), 2.59$ (d, $\mathrm{J}=16.8 \mathrm{~Hz}, 2 \mathrm{H}), 2.55$ (d, J=16.8 Hz, $2 \mathrm{H}), 0.18$ (s, $18 \mathrm{H}) \mathrm{ppm} ;{ }^{13} \mathrm{C} \mathrm{NMR}\left(100 \mathrm{MHz}, \mathrm{CDCl}_{3}\right): \delta=155.9$, 132.6, 129.0, 123.2, 120.4, 117.6, 101.9, 89.5, 75.8, 40.4, 30.7, 0.1 ppm; IR $\left(\mathrm{CHCl}_{3}\right): \mathrm{v}=3548,3340$, $3040,2962,2905,2253,2174,1943,1796,1584,1488,1376,1249,1026,844 \mathrm{~cm}^{-1}$; MS (Cl-NH $)_{3}$ : $\mathrm{m} / \mathrm{z}(\%): 376(100)\left[\left(\mathrm{M}+\mathrm{NH}_{4}\right)^{+}\right], 359(20)\left[(\mathrm{M}+\mathrm{H})^{+}\right]$; elemental analysis calcd (\%) for $\mathrm{C}_{20} \mathrm{H}_{30} \mathrm{O}_{2} \mathrm{Si}_{2}: \mathrm{C}$ 66.98, H 8.43; found: C 67.02, H 8.58. 
2-[2-Hydroxy-2-(2-propynyl)-4-pentynyl)phenol (SI9)-typical procedure for the desilylation of alkynes: At ambient temperature, a solution of SI6 (4.59 g, $12.8 \mathrm{mmol})$ and THF (10 mL) was added to a solution of TBAF $3 \mathrm{H}_{2} \mathrm{O}(1.21 \mathrm{~g}, 3.84 \mathrm{mmol})$ in THF $(20 \mathrm{~mL})$ and the reaction mixture was maintained at this temperature for $15 \mathrm{~h}$. Dilution with $t$-butyl methyl ether $(180 \mathrm{~mL})$ was followed by washing with $\mathrm{H}_{2} \mathrm{O}(90 \mathrm{~mL})$ and brine $(90 \mathrm{~mL})$. After drying $\left(\mathrm{MgSO}_{4}\right)$, the solvents were evaporated and the resulting residue was purified by flash column chromatography on silica gel (cyclohexane/t-butyl methyl ether $3: 1)$ to give SI9 $(2.34 \mathrm{~g}, 85 \%)$ as a colorless solid. $R_{\mathrm{f}}=0.22$ (cyclohexane/t-butyl methyl ether 2:1); m.p. $64-66^{\circ} \mathrm{C} ;{ }^{1} \mathrm{H}$ NMR $\left(500 \mathrm{MHz}, \mathrm{CDCl}_{3}\right.$ ): $\delta=7.86$ (br s, $1 \mathrm{H}$ ), 7.16 (ddd, J=7.7, 7.7, $1.8 \mathrm{~Hz}$, $1 \mathrm{H}$ ), 7.10 (dd, J=7.5, $1.7 \mathrm{~Hz}, 1 \mathrm{H}$ ), 6.92 (dd, J=8.0, $1.2 \mathrm{~Hz}, 1 \mathrm{H}$ ), 6.84 (ddd, J=7.5, 7.5, $1.3 \mathrm{~Hz}, 1 \mathrm{H}$ ), 3.14 (br s, 1 H), 3.01 (s, 2 H), 2.59 (dd, J=16.6, 2,6 Hz, 2 H), 2.55 (dd, J=16.7, 2.7 Hz, 2 H), 2.15 (dd, $\mathrm{J}=2.7,2.6 \mathrm{~Hz}, 2 \mathrm{H}) \mathrm{ppm} ;{ }^{13} \mathrm{C} \mathrm{NMR}\left(125 \mathrm{MHz}, \mathrm{CDCl}_{3}\right): \delta=155.7,132.6,129.0,123.0,120.5,117.6$, 79.5, 75.9, 72.5, 40.3, 29.2 ppm; IR $\left(\mathrm{CHCl}_{3}\right)$ : v=3628, 3307, 3028, 2977, 2401, 2361, 1521, 1422, 1221, 1047, $929 \mathrm{~cm}^{-1}$; MS (EI): $\mathrm{m} / \mathrm{z}(\%): 214(31)\left[\mathrm{M}^{+}\right]$; elemental analysis calcd (\%) for $\mathrm{C}_{14} \mathrm{H}_{14} \mathrm{O}_{2}: \mathrm{C}$ 78.48, H 6.59; found: C 78.25, H 6.58 .

\section{2-[2-Hydroxy-5-phenyl-2-(3-phenyl-2-propynyl)-4-pentynyl]phenol (SI12)-typical procedure for} a two-fold Sonogashira cross-coupling: At ambient temperature, $\mathrm{Et}_{3} \mathrm{~N}(10 \mathrm{~mL})$ was added to a Schlenk flask charged with $\left(\mathrm{Ph}_{3} \mathrm{P}\right)_{2} \mathrm{PdCl}_{2}(0.061 \mathrm{~g}, 0.087 \mathrm{mmol}, 1.0 \mathrm{~mol} \%)$ and Cul $(8.3 \mathrm{mg}, 0.043$ $\mathrm{mmol}, 0.50 \mathrm{~mol} \%)$. After $5 \mathrm{~min}$, a solution of $\mathrm{SI} 9(1.85 \mathrm{~g}, 8.68 \mathrm{mmol})$ with $\mathrm{Et}_{3} \mathrm{~N}(15 \mathrm{~mL})$ and $\mathrm{Phl}(2.00$ $\mathrm{mL}, 3.54 \mathrm{~g}, 17.4 \mathrm{mmol}$ ) were subsequently added via syringe and the reaction mixture was maintained under vigorous stirring at room temperature for $40 \mathrm{~h}$, whereupon a voluminous precipitate formed. The mixture was diluted with $t$-butyl methyl ether $(250 \mathrm{~mL})$, washed with aq $\mathrm{HCl}(2 \mathrm{M}, 2 \times 100 \mathrm{ml})$ and saturated aq $\mathrm{NaHCO}_{3}(100 \mathrm{~mL})$, and dried over $\mathrm{MgSO}_{4}$. Evaporation of the solvents under reduced pressure and purification of the resulting residue by flash column chromatography on silica gel (cyclohexane/t-butyl methyl ether 4:1) gave the desired cross-coupling product SI12 (2.98 g, 94\%) as a colorless solid. $R_{\mathrm{f}}=0.32$ (cyclohexane/t-butyl methyl ether $3: 1$ ); m.p. $88-90^{\circ} \mathrm{C} ;{ }^{1} \mathrm{H}$ NMR $(500 \mathrm{MHz}$, $\left.\mathrm{CDCl}_{3}\right): \delta=8.04(\mathrm{~s}, 1 \mathrm{H}), 7.42-7.47(\mathrm{~m}, 4 \mathrm{H}), 7.29-7.33(\mathrm{~m}, 6 \mathrm{H}), 7.15-7.22(\mathrm{~m}, 2 \mathrm{H}), 6.97$ (dd, J=8.0, $1.1 \mathrm{~Hz}, 1 \mathrm{H}), 6.88$ (ddd, J=7.4, 7.4, $1.1 \mathrm{~Hz}, 1 \mathrm{H}), 3.23(\mathrm{~s}, 1 \mathrm{H}), 3.14(\mathrm{~s}, 2 \mathrm{H}), 2.87(\mathrm{~d}, \mathrm{~J}=16.8 \mathrm{~Hz}, 2 \mathrm{H}$ ), $2.83\left(\mathrm{~d}, \mathrm{~J}=16.8 \mathrm{~Hz}, 2 \mathrm{H}\right.$ ) ppm; ${ }^{13} \mathrm{C} \mathrm{NMR}\left(125 \mathrm{MHz}, \mathrm{CDCl}_{3}\right): \delta=155.9,132.7,131.8,129.0,128.4$, 128.4, 123.3, 123.0, 120.5, 117.7, 84.8, 84.6, 76.6, 40.9, 30.5 ppm; IR $\left(\mathrm{CHCl}_{3}\right): \mathrm{v}=3554,3336,3024$, 2977, 2401, 2361, 1585, 1490, 1424, 1224, 1054, $797 \mathrm{~cm}^{-1}$; MS (Cl-isobutene): $\mathrm{m} / \mathrm{z}$ (\%): 367 (63) $\left[(\mathrm{M}+\mathrm{H})^{+}\right]$; elemental analysis calcd (\%) for $\mathrm{C}_{26} \mathrm{H}_{22} \mathrm{O}_{2}$ : C 85.22, $\mathrm{H}$ 6.05; found: C 84.99, H 6.02.

(E,E)-2-[2-Hydroxy-5-phenyl-2-(3-phenyl-2-propenyl)-4-pentenyl]phenol [(E,E)-SI15]: A Schlenk flask was charged with $\mathrm{LiAlH}_{4}(0.940 \mathrm{~g}, 24.8 \mathrm{mmol})$, cooled to $0^{\circ} \mathrm{C}$ and dry THF $(20 \mathrm{~mL})$ was added. After $10 \mathrm{~min}$, a solution of diyne SI12 $(1.21 \mathrm{~g}, 3.30 \mathrm{mmol})$ and dry THF $(10 \mathrm{~mL})$ was added dropwise under formation of $\mathrm{H}_{2}$. The reaction mixture was then allowed to warm to room temperature overnight and maintained at ambient temperature for two days for completion. At $0^{\circ} \mathrm{C}$, the reaction was carefully quenched by slow addition of aq $\mathrm{NaOH}(2.5 \mathrm{M}, 2 \mathrm{~mL})$. The gelatinous mass was poured into $t$-butyl methyl ether $(180 \mathrm{~mL})$ and aq $\mathrm{HCl}(2 \mathrm{M}, 120 \mathrm{~mL})$, the aqueous layer was extracted with $t$-butyl methyl ether $(60 \mathrm{~mL})$, and the combined organic phases were washed with saturated aq $\mathrm{NaHCO}_{3}(60 \mathrm{~mL})$. 
After drying $\left(\mathrm{MgSO}_{4}\right)$, the solvents were evaporated in vacuo and the residue was purified by flash column chromatography on silica gel (cyclohexane/t-butyl methyl ether 6:1) affording the diene $(E, E)$ SI15 $(1.15 \mathrm{~g}, 94 \%)$ as a colorless oil. The $E, Z$-ratio was determined from the ${ }^{1} \mathrm{H}$ NMR spectra ( $E: Z \geq 99: 1) . R_{\mathrm{f}}=0.33$ (cyclohexane/t-butyl methyl ether $3: 1$ ); ${ }^{1} \mathrm{H}$ NMR $\left(400 \mathrm{MHz}, \mathrm{CDCl}_{3}\right): \delta=8.53$ (br s, 1 H), 7.37-7.41 (m, $4 \mathrm{H}), 7.32-7.36(\mathrm{~m}, 4 \mathrm{H}), 7.27\left(\mathrm{~m}_{\mathrm{c}}, 2 \mathrm{H}\right), 7.21$ (ddd, J=8.0, 7.4, 1.7 Hz, $\left.1 \mathrm{H}\right), 7.06$ (dd, J=7.5, $1.6 \mathrm{~Hz}, 1 \mathrm{H}$ ), 6.98 (dd, J=8.1, $1.3 \mathrm{~Hz}, 1 \mathrm{H}$ ), 6.87 (ddd, J=7.4, 7.4, $1.3 \mathrm{~Hz}, 1 \mathrm{H}), 6.51$ (d, J=15.8 Hz, $2 \mathrm{H}$ ), 6.30 (ddd, J=15.6, 8.2, 7.2 Hz, $2 \mathrm{H}$ ), 2.97 (s, $2 \mathrm{H}$ ), 2.71 (br s, $1 \mathrm{H}$ ), 2.55 (ddd, J=13.9, 7.0, $1.3 \mathrm{~Hz}, 2 \mathrm{H}$ ), 2.44 (ddd, J=13.9, 8.1, $1.1 \mathrm{~Hz}, 2 \mathrm{H}$ ) ppm; ${ }^{13} \mathrm{C}$ NMR $\left(100 \mathrm{MHz}, \mathrm{CDCl}_{3}\right): \delta=156.0$, 136.9, 135.1, 132.7, 128.7, 127.8, 126.3, 124.0, 123.9, 120.2, 117.6, 77.5, 43.0, 42.8 ppm; IR $\left(\mathrm{CHCl}_{3}\right)$ : $\mathrm{v}=3618,3293,3027,1584,1489,1436,1238,1198,1044,970 \mathrm{~cm}^{-1} ; \mathrm{MS}\left(\mathrm{Cl}-\mathrm{NH}_{3}\right): \mathrm{m} / \mathrm{z}(\%): 388(29)$ $\left[\left(\mathrm{M}+\mathrm{NH}_{4}\right)^{+}\right], 371(15)\left[(\mathrm{M}+\mathrm{H})^{+}\right]$; elemental analysis calcd (\%) for $\mathrm{C}_{26} \mathrm{H}_{26} \mathrm{O}_{2}: \mathrm{C} 84.29, \mathrm{H}$ 7.07; found: C 84.46, H 7.16.

$(E, E)$-Trifluoromethanesulfonic acid 2-[2-hydroxy-5-phenyl-2-(3-phenyl-2-propenyl)-4pentenyl]phenyl ester $[(E, E)-9]$-typical procedure for the chemoselective triflation of phenols: At room temperature, a solution of $(E, E)$-SI15 $(0.616 \mathrm{~g}, 1.66 \mathrm{mmol})$ in dry DMF $(8 \mathrm{~mL})$ was added to a mixture of $\mathrm{PhNTf}_{2}(0.594 \mathrm{~g}, 1.66 \mathrm{mmol})$ and $\mathrm{Cs}_{2} \mathrm{CO}_{3}(0.542 \mathrm{~g}, 1.66 \mathrm{mmol})$ in dry DMF (6 mL). After 15 $\mathrm{h}$, the reaction mixture was diluted with $t$-butyl methyl ether $(180 \mathrm{~mL})$ followed by several washes with $\mathrm{H}_{2} \mathrm{O}(5 \times 50 \mathrm{~mL})$ for complete removal of DMF. The organic layer was dried over $\mathrm{MgSO}_{4}$ and evaporated. The resulting residue was purified by flash column chromatography on silica gel (cyclohexane/t-butyl methyl ether $9: 1)$ affording $(E, E)-9(0.786 \mathrm{~g}, 94 \%)$ as a colorless oil. $R_{\mathrm{f}}=0.31$ (cyclohexane/t-butyl methyl ether 3:1); ${ }^{1} \mathrm{H}$ NMR $\left(400 \mathrm{MHz}, \mathrm{CDCl}_{3}\right)$ : $\delta=7.52-7.57(\mathrm{~m}, 1 \mathrm{H}), 7.16-7.43$ $(\mathrm{m}, 13 \mathrm{H}$ ), 6.51 (d, J=15.9 Hz, $2 \mathrm{H}$ ), 6.30 (ddd, J=15.6, 7.6, 7.6 Hz, $2 \mathrm{H}$ ), 3.03 (s, $2 \mathrm{H}$ ), 2.49 (ddd, $\mathrm{J}=14.0,7.1,0.9 \mathrm{~Hz}, 2 \mathrm{H}), 2.41$ (ddd, J=14.6, 6.9, $0.9 \mathrm{~Hz}, 2 \mathrm{H}), 1.80(\mathrm{~s}, 1 \mathrm{H}) \mathrm{ppm} ;{ }^{13} \mathrm{C} \mathrm{NMR}(100 \mathrm{MHz}$, $\left.\mathrm{CDCl}_{3}\right): \delta=149.0,137.2,134.6,133.8,130.6,128.6,128.6,128.5,127.5,126.3,124.6,121.4,118.7$ (q, J(C,F)=320 Hz), 74.8, 43.3, 39.6 ppm; IR $\left(\mathrm{CHCl}_{3}\right): \mathrm{v}=3621,3016,2977,1521,1493,1420,1206$, 1141, 1047, $928 \mathrm{~cm}^{-1}$; MS (APCl): $\mathrm{m} / \mathrm{z}(\%): 502(87)\left[\mathrm{M}^{+}\right]$; elemental analysis calcd (\%) for $\mathrm{C}_{27} \mathrm{H}_{25} \mathrm{~F}_{3} \mathrm{O}_{4} \mathrm{~S}$ : C 64.53, H 5.01, S 6.38; found: C 64.80, H 5.14, S 6.51.

$(E, E)$-Trifluoromethanesulfonic acid 2-[2-methoxy-5-phenyl-2-(3-phenyl-2-propenyl)-4pentenyl]phenyl ester $[(E, E)-11]$-typical procedure for the alkylation of tertiary alcohols with Meerwein's salt: At room temperature, a solution of the tertiary alcohol $(E, E)-9(0.440 \mathrm{~g}, 0.876 \mathrm{mmol})$, 1,8-bis(dimethylamino)naphthalene $(0.939 \mathrm{~g}, 4.38 \mathrm{mmol})$ and dry $\mathrm{CH}_{2} \mathrm{Cl}_{2}(12 \mathrm{~mL})$ was added to a suspension of trimethyloxonium tetrafluoro borate $(0.650 \mathrm{~g}, 4.38 \mathrm{mmol})$ in dry $\mathrm{CH}_{2} \mathrm{Cl}_{2}(5 \mathrm{~mL})$, and the resulting mixture was vigorously stirred overnight. The reaction mixture was diluted with $t$-butyl methyl ether $(140 \mathrm{~mL})$, washed with aq $\mathrm{HCl}(2 \mathrm{M}, 2 \times 50 \mathrm{~mL})$ and saturated aq $\mathrm{NaHCO}_{3}(50 \mathrm{~mL})$ and dried $\left(\mathrm{MgSO}_{4}\right)$. After evaporation of the solvents, the residue was purified by flash column chromatography on silica gel (cyclohexane/t-butyl methyl ether $98: 2 \rightarrow 5: 1$ ) to give the desired methyl ether $(E, E)-11$ $(0.220 \mathrm{~g}, 49 \%)$ along with unreacted alcohol $(E, E)-9(0.189 \mathrm{~g}, 43 \%)$ as colorless oils. $R_{\mathrm{f}}=0.49$ (cyclohexane/t-butyl methyl ether 9:1); ${ }^{1} \mathrm{H}$ NMR $\left(500 \mathrm{MHz}, \mathrm{CDCl}_{3}\right): \delta=7.62$ (dd, J=7.6, $1.9 \mathrm{~Hz}, 1 \mathrm{H}$ ), 7.36-7.40 (m, $4 \mathrm{H}), 7.30-7.36(\mathrm{~m}, 6 \mathrm{H}), 7.23-7.28(\mathrm{~m}, 3 \mathrm{H}), 6.51$ (d, J=15.8 Hz, $2 \mathrm{H}$ ), 6.26 (ddd, 
$\mathrm{J}=15.8,7.2,7.2 \mathrm{~Hz}, 2 \mathrm{H}), 3.44(\mathrm{~s}, 3 \mathrm{H}), 3.05(\mathrm{~s}, 2 \mathrm{H}), 2.51\left(\mathrm{~m}_{\mathrm{c}}, 4 \mathrm{H}\right) \mathrm{ppm} ;{ }^{13} \mathrm{C} \mathrm{NMR}\left(125 \mathrm{MHz}, \mathrm{CDCl}_{3}\right)$ : $\delta=148.8,137.5,133.4,133.2,130.9,128.6,128.3,128.0,127.3,126.2,125.0,121.3,118.6$ (q, $J(C, F)=320 \mathrm{~Hz}), 79.4,49.5,39.2,33.9 \mathrm{ppm} ; \mathrm{IR}\left(\mathrm{CDCl}_{3}\right): \mathrm{v}=3030,2940,2832,1599,1490,1419,1219$, 1141, 1077, $905 \mathrm{~cm}^{-1}$; MS (Cl-NH $): m / z(\%): 534(100)\left[\left(\mathrm{M}+\mathrm{NH}_{4}\right)^{+}\right], 503(12)\left[(\mathrm{M}+\mathrm{H})^{+}\right]$; HRMS (EI): $\mathrm{m} / \mathrm{z}$ calcd for $\mathrm{C}_{19} \mathrm{H}_{18} \mathrm{~F}_{3} \mathrm{O}_{4} \mathrm{~S}\left[\left(\mathrm{M}-\mathrm{C}_{9} \mathrm{H}_{9}\right)^{+}\right]: 399.08770$; found: 399.08778 .

$(R, E, E)$-4-Benzylidene-2-(3-phenyl-2-propenyl)-1,2,3,4-tetrahydro-2-naphthalenol $\quad[(R, E, E)-13]-$ typical procedure for desymmetrizing Heck cyclizations: At room temperature, a sealed tube was charged with $\mathrm{Pd}(\mathrm{OAc})_{2}(4.5 \mathrm{mg}, 0.020 \mathrm{mmol}, 5.0 \mathrm{~mol} \%),(R)$-BINAP $(0.019 \mathrm{~g}, 0.030 \mathrm{mmol}, 7.5 \mathrm{~mol} \%)$ and $\mathrm{K}_{2} \mathrm{CO}_{3}(0.220 \mathrm{~g}, 1.59 \mathrm{mmol})$ under a static pressure of argon. A solution of triflate $(E, E)-9(0.200$ $\mathrm{g}, 0.398 \mathrm{mmol}$ ) and degassed dry toluene $(5.5 \mathrm{~mL})$ was added and the tube was sealed. The resulting suspension was vigorously stirred for $1 \mathrm{~h}$ until the formation of a red homogenous solution except for the solid base and then heated at $60^{\circ} \mathrm{C}$ for $36 \mathrm{~h}$. At room temperature, a small amount of silica gel was added and after evaporation of the solvents, the crude product on silical gel was directly purified by flash column chromatography on silica gel (cyclohexane/t-butyl methyl ether 9:1) giving $(R, E, E)-13$ $(0.115 \mathrm{~g}, 82 \%)$ as a colorless oil. The enantiomeric excess was determined by HPLC analysis $(94 \%$ ee). As determined from the ${ }^{1} \mathrm{H}$ NMR spectra, $(R, E, E)-13$ was isomerically pure. $R_{\mathrm{f}}=0.36$ (cyclohexane/t-butyl methyl ether 3:1); HPLC (Daicel Chiracel AD, $n$-heptane/i-PrOH 9:1, flow rate=1 $\mathrm{mL} / \mathrm{min}, \lambda=295 \mathrm{~nm}$, column temperature: $\left.15^{\circ} \mathrm{C}\right): t_{\mathrm{R}}=18.19 \min (R), t_{\mathrm{R}}=25.75 \mathrm{~min}(\mathrm{~S}) ;[\alpha]_{\mathrm{D}}{ }^{20}=+20.9$ $\left(c=0.390, \mathrm{CHCl}_{3}, 92 \%\right.$ ee); ${ }^{1} \mathrm{H}$ NMR $\left(400 \mathrm{MHz}, \mathrm{CDCl}_{3}\right): \delta=7.73-7.77(\mathrm{~m}, 1 \mathrm{H}), 7.36-7.39(\mathrm{~m}, 4 \mathrm{H})$, 7.23-7.31 (m, $6 \mathrm{H}), 7.17-7.21(\mathrm{~m}, 1 \mathrm{H}), 7.13-7.17$ (m, $3 \mathrm{H}), 6.39$ (d, J=15.9 Hz, $1 \mathrm{H}), 6.12$ (ddd, $\mathrm{J}=15.8,8.3,7.0 \mathrm{~Hz}, 1 \mathrm{H}$ ), 3.01 (s, $2 \mathrm{H}$ ), 2.91 (s, $2 \mathrm{H}$ ), 2.47 (ddd, J=13.9, 7.0, $1.4 \mathrm{~Hz}, 1 \mathrm{H}$ ), 2.35 (ddd, $\mathrm{J}=13.9,8.2,1.2 \mathrm{~Hz}, 1 \mathrm{H}), 1.88(\mathrm{~s}, 1 \mathrm{H}) \mathrm{ppm} ;{ }^{13} \mathrm{C} \mathrm{NMR}\left(100 \mathrm{MHz}, \mathrm{CDCl}_{3}\right): \delta=137.7,137.1,135.0$, $134.9,134.8,134.1,130.0,129.5,128.5,128.4,128.1,127.3,126.9,126.8,126.7,126.2,124.9$, 124.2, 71.9, 43.7, 42.6, 39.6 ppm; IR $\left(\mathrm{CHCl}_{3}\right): \mathrm{v}=3620,3015,2977,1522,1421,1233,1201,1047$, $929 \mathrm{~cm}^{-1}$; MS (EI): $\mathrm{m} / \mathrm{z}(\%): 352(19)\left[\mathrm{M}^{+}\right]$; elemental analysis calcd (\%) for $\mathrm{C}_{26} \mathrm{H}_{24} \mathrm{O}: \mathrm{C}$ 88.60, H 6.86; found: C 88.40, H 6.91 .

\section{$4 \quad$ Characterization Data}

2,2-Dimethylbenzo[1,3]dioxin-4-one (SI1): At $0^{\circ} \mathrm{C}$, to a solution of salicylic acid (11.1 g, $80.2 \mathrm{mmol}$ ) and dry acetone $(40 \mathrm{~mL})$ were added $\mathrm{Ac}_{2} \mathrm{O}(8.60 \mathrm{~mL}, 9.30 \mathrm{~g}, 92.0 \mathrm{mmol})$ and $\mathrm{H}_{2} \mathrm{SO}_{4}(0.26 \mathrm{~mL})$. After $20 \mathrm{~h}$ at $0^{\circ} \mathrm{C}$, the reaction mixture was diluted with $t$-butyl methyl ether $(300 \mathrm{~mL})$, washed with aq $\mathrm{NaOH}$ $(2 \mathrm{M}, 2 \times 80 \mathrm{~mL})$ and dried over $\mathrm{Na}_{2} \mathrm{SO}_{4}$. The solvents were removed under reduced pressure and the crude product was recrystallized from cyclohexane to afford SI1 $(5.72 \mathrm{~g}, 40 \%)$ as white crystals. $R_{\mathrm{f}}=0.52$ (cyclohexane/t-butyl methyl ether 3:1); m.p. $57-58^{\circ} \mathrm{C}$; ${ }^{1} \mathrm{H}$ NMR $\left(400 \mathrm{MHz}, \mathrm{CDCl}_{3}\right): \delta=7.94$ (dd, $\mathrm{J}=7.7,1.7 \mathrm{~Hz}, 1 \mathrm{H}$ ), 7.54 (ddd, J=7.8, 7.7, 1.7 Hz, $1 \mathrm{H}$ ), 7.10 (dd, J=7.7, 7.7 Hz, $1 \mathrm{H}$ ), 6.95 (d, J=8.2, 1 $\mathrm{H}), 1.76(\mathrm{~s}, 6 \mathrm{H}) \mathrm{ppm} ;{ }^{13} \mathrm{C}$ NMR $\left(100 \mathrm{MHz}, \mathrm{CDCl}_{3}\right): \delta=161.1,156.1,136.4,129.7,122.7,117.2,113.7$, 106.4, 25.9 ppm; IR $\left(\mathrm{CHCl}_{3}\right)$ : v=3685, 3022, 2255, 1734, 1615, 1522, 1473, 1427, 1387, 1312, 1213, 1057, $902 \mathrm{~cm}^{-1}$; MS (EI): $\mathrm{m} / \mathrm{z}(\%): 178(37)\left[\mathrm{M}^{+}\right]$; elemental analysis calcd (\%) for $\mathrm{C}_{10} \mathrm{H}_{10} \mathrm{O}_{3}: \mathrm{C} 67.41$, H 5.66; found: C 67.24, H 5.51 . 
2-[1-Hydroxy-4-trimethylsilyl-1-(3-trimethylsilyl-2-propynyl)-3-butynyl]phenol (SI5): SI5 was prepared according to the protocol described for SI6; formation of acetone during the reaction required an additional equivalent of SI4. Reaction of zinc reagent SI4 generated from zinc dust (5.23 g, 80.0 $\mathrm{mmol}), 1,2$-dibromoethane $(0.80 \mathrm{~mL}), \mathrm{Me}_{3} \mathrm{SiCl}(0.40 \mathrm{~mL})$ and 3-bromo-1-trimethylsilyl-1-propyne $(7.70$ g, $40.0 \mathrm{mmol})$ with SI1 $(1.78 \mathrm{~g}, 10.0 \mathrm{mmol})$ in dry THF (100 mL) gave diyne SI5 $(3.16 \mathrm{~g}, 92 \%)$ as a colorless oil. $R_{\mathrm{f}}=0.57$ (cyclohexane/t-butyl methyl ether $\left.3: 1\right) ;{ }^{1} \mathrm{H}$ NMR $\left(400 \mathrm{MHz}, \mathrm{CDCl}_{3}\right): \delta=8.59$ (s, 1 H), 7.18 (ddd, J=7.7, 7.7, $1.7 \mathrm{~Hz}, 1 \mathrm{H}$ ), 7.08 (dd, J=7.9, $1.5 \mathrm{~Hz}, 1 \mathrm{H}$ ), 6.88 (dd, J=8.2, $1.3 \mathrm{~Hz}, 1 \mathrm{H}$ ), 6.82 (ddd, J=7.6, 7.6, $1.1 \mathrm{~Hz}, 1 \mathrm{H}$ ), 3.38 (s, $1 \mathrm{H}$ ), 3.00 (d, J=17.2 Hz, $2 \mathrm{H}$ ), 2.98 (d, J=16.8 Hz, $2 \mathrm{H}$ ), $0.12(\mathrm{~s}, 18 \mathrm{H}) \mathrm{ppm} ;{ }^{13} \mathrm{C}$ NMR $\left(100 \mathrm{MHz}, \mathrm{CDCl}_{3}\right): \delta=156.1,129.6,126.9,125.8,119.3,117.9,101.4$, 89.9, 77.6, 32.3, 0.0 ppm; IR (neat): v=3367, 2960, 2900, 2178, 1618, 1585, 1493, 1423, 1250, 1026 , $843 \mathrm{~cm}^{-1}$; MS (Cl-NH $\mathrm{NH}_{3}: \mathrm{m} / \mathrm{z}(\%): 362(100)\left[\left(\mathrm{M}+\mathrm{NH}_{4}\right)^{+}\right]$; elemental analysis calcd (\%) for $\mathrm{C}_{19} \mathrm{H}_{28} \mathrm{O}_{2} \mathrm{Si}_{2}$ : C 66.22, H 8.19; found: C 66.08, H 8.25.

4-(2-Bromobenzyl)-1,7-bistrimethylsilyl-1,6-heptadiyn-4-ol (SI7): Treatment of (2bromophenyl)acetic acid ethyl ester (SI3) (2.60 g. $10.7 \mathrm{mmol})$ with zinc reagent SI4-generated from zinc dust $(4.20 \mathrm{~g}, 64.2 \mathrm{mmol})$, 1,2-dibromoethane $(0.64 \mathrm{~mL}), \mathrm{Me}_{3} \mathrm{SiCl}(0.32 \mathrm{~mL})$ and 3-bromo-1trimethylsilyl-1-propyne $(5.95 \mathrm{~g}, 31.1 \mathrm{mmol})$ in dry THF $(75 \mathrm{~mL})$ —gave the desired diyne SI7 $(3.79 \mathrm{~g}$, $84 \%$ ) as a colorless oil. $R_{\mathrm{f}}=0.57$ (cyclohexane/t-butyl methyl ether $9: 1$ ); ${ }^{1} \mathrm{H}$ NMR $\left(400 \mathrm{MHz}, \mathrm{CDCl}_{3}\right.$ ): $\delta=7.55$ (dd, J=8.1, 1.3 Hz, $1 \mathrm{H}$ ), 7.47 (dd, J=7.7, 1.7 Hz, $1 \mathrm{H}$ ), 7.27 (ddd, J=7.5, 7.5, 1.3 Hz, $1 \mathrm{H}$ ), 7.10 (ddd, J=8.0, 7.4, 1.8 Hz, $1 \mathrm{H}$ ), 3.29 (s, $2 \mathrm{H}$ ), 2.62 (d, J=16.9 Hz, $2 \mathrm{H}$ ), 2.54 (d, J=16.8 Hz, $2 \mathrm{H}$ ), 2.47 (s, $1 \mathrm{H}), 0.18(\mathrm{~s}, 18 \mathrm{H}) \mathrm{ppm} ;{ }^{13} \mathrm{C} \mathrm{NMR}\left(100 \mathrm{MHz}, \mathrm{CDCl}_{3}\right): \delta=136.6,133.0,132.7,128.5,127.3,126.4$, 102.6, 89.1, 74.2, 42.2, 31.3, 0.1 ppm; IR $\left(\mathrm{CDCl}_{3}\right)$ : v=3557, 3062, 2962, 2901, 2248, 2174, 1950,

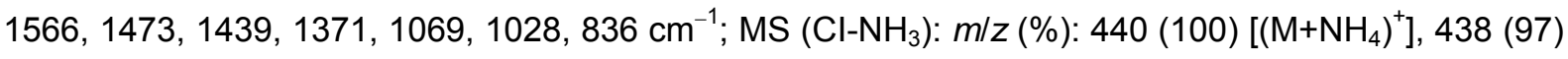
$\left[\left(\mathrm{M}^{+}+\mathrm{NH}_{4}\right)^{+}\right]$; elemental analysis calcd (\%) for $\mathrm{C}_{20} \mathrm{H}_{29} \mathrm{BrOSi}_{2}$ : C 56.99, $\mathrm{H}$ 6.93; found: C 57.26, H 6.92.

2-[1-Hydroxy-1-(2-propynyl)-3-butynyl]phenol (SI8): In analogy to the desilylation of SI6, reaction of SI5 (0.830 g, $2.41 \mathrm{mmol})$ with TBAF $3 \mathrm{H}_{2} \mathrm{O}(0.280 \mathrm{~g}, 0.800 \mathrm{mmol})$ in THF $(12 \mathrm{~mL})$ afforded desired SI8 $(0.377 \mathrm{~g}, 78 \%)$ as a colourless oil. $R_{\mathrm{f}}=0.22$ (cyclohexane/t-butyl methyl ether $\left.3: 1\right) ;{ }^{1} \mathrm{H}$ NMR $(400 \mathrm{MHz}$, $\mathrm{CDCl}_{3}$ ): $\delta=7.21$ (ddd, J=8.0, 8.0, $1.3 \mathrm{~Hz}, 1 \mathrm{H}$ ), 7.14 (dd, J=8.0, $1.5 \mathrm{~Hz}, 1 \mathrm{H}$ ), 6.90 (dd, J=8.2, $1.3 \mathrm{~Hz}, 1$ H), 6.87 (ddd, J=8.0, 8.0, $1.3 \mathrm{~Hz}, 1 \mathrm{H}$ ), 3.01 (dd, J=17.0, 2.8 Hz, $2 \mathrm{H}$ ), 2.98 (dd, J=17.0, 2.8 Hz, $2 \mathrm{H}$ ), 2.11 (dd, J=2.6, $2.6 \mathrm{~Hz}, 2 \mathrm{H}$ ) ppm; ${ }^{13} \mathrm{C}$ NMR $\left(100 \mathrm{MHz}, \mathrm{CDCl}_{3}\right): \delta=155.9,129.9,126.7,125.7,119.7$, 118.1, 79.0, 77.4, 72.9, 30.6 ppm; IR $\left(\mathrm{CHCl}_{3}\right): \mathrm{v}=3539,3372,3307,3013,2922,2122,1584,1491$, 1428, 1372, 1243, $1041 \mathrm{~cm}^{-1}$; MS (EI): $\mathrm{m} / \mathrm{z}(\%): 200(24)\left[\mathrm{M}^{+}\right]$; elemental analysis calcd (\%) for $\mathrm{C}_{13} \mathrm{H}_{12} \mathrm{O}_{2}:$ C 77.98, H 6.04; found: C 77.68, H 6.10.

4-(2-Bromobenzyl)-1,6-heptadiyn-4-ol (SI10): According to the preparation of SI9, the reaction diyne SI7 $(0.970 \mathrm{~g}, 2.30 \mathrm{mmol})$ with TBAF $3 \mathrm{H}_{2} \mathrm{O}(0.360 \mathrm{~g}, 1.14 \mathrm{mmol})$ in THF $(10 \mathrm{~mL})$ gave SI10 $(0.576 \mathrm{~g}$, $90 \%$ ) as a colorless oil. $R_{\mathrm{f}}=0.33$ (cyclohexane/t-butyl methyl ether $\left.4: 1\right) ;{ }^{1} \mathrm{H}$ NMR $\left(500 \mathrm{MHz}, \mathrm{CDCl}_{3}\right)$ : $\delta=7.56(\mathrm{dd}, \mathrm{J}=8.1,1.4 \mathrm{~Hz}, 1 \mathrm{H}$ ), 7.47 (dd, J=7.7, 1.7 Hz, $1 \mathrm{H}$ ), 7.28 (ddd, J=7.5, 7.5, 1.3 Hz, $1 \mathrm{H}$ ), 7.12 (ddd, J=7.7, 7.7, $1.7 \mathrm{~Hz}, 1 \mathrm{H}$ ), 3.28 (s, $2 \mathrm{H}$ ), 2.62 (dd, J=16.7, $2.7 \mathrm{~Hz}, 2 \mathrm{H}$ ), 2.54 (dd, J=16.8, $2.7 \mathrm{~Hz}, 2$ 
$\mathrm{H}), 2.38(\mathrm{~s}, 1 \mathrm{H}), 2.16(\mathrm{dd}, \mathrm{J}=2.7,2.7 \mathrm{~Hz}, 2 \mathrm{H}) \mathrm{ppm} ;{ }^{13} \mathrm{C} \mathrm{NMR}\left(125 \mathrm{MHz}, \mathrm{CDCl}_{3}\right): \delta=136.2,133.1$, 132.7, 128.6, 127.4, 126.2, 80.1, 74.0, 72.3, 42.4, 29.7 ppm; IR $\left(\mathrm{CHCl}_{3}\right)$ : v=3687, 3567, 3308, 3021, 2120, 1806, 1700, 1651, 1521, 1425, 1211, 1051, $929 \mathrm{~cm}^{-1} ; \mathrm{MS}\left(\mathrm{Cl}-\mathrm{NH}_{3}\right): \mathrm{m} / \mathrm{z}$ (\%): 296 (95) $\left[\left(\mathrm{M}+\mathrm{NH}_{4}\right)^{+}\right], 294(100)\left[\left(\mathrm{M}+\mathrm{NH}_{4}\right)^{+}\right]$; elemental analysis calcd (\%) for $\mathrm{C}_{14} \mathrm{H}_{13} \mathrm{BrO}: \mathrm{C}$ 60.67, H 4.73; found: C $60.54, \mathrm{H} 4.75$.

2-[1-Hydroxy-4-phenyl-1-(3-phenyl-2-propynyl)-3-butynyl]phenol (SI11): Following the procedure described for SI12, two-fold Sonogashira cross-coupling of SI8 $(0.300 \mathrm{~g}, 1.50 \mathrm{mmol})$ with $\mathrm{Phl}(0.340$ $\mathrm{mL}, 0.610 \mathrm{~g}, 3.00 \mathrm{mmol})$ in the presence of $\left(\mathrm{Ph}_{3} \mathrm{P}\right)_{2} \mathrm{PdCl}_{2}(11 \mathrm{mg}, 0.016 \mathrm{mmol}, 1.0 \mathrm{~mol} \%)$ and Cul (1.4 $\mathrm{mg}, 7.5 \mu \mathrm{mol}, 0.50 \mathrm{~mol} \%)$ in $\mathrm{Et}_{3} \mathrm{~N}(6 \mathrm{~mL})$ gave SI11 $(4.79 \mathrm{~g}, 91 \%)$ as a colorless oil. $R_{\mathrm{f}}=0.35$ (cyclohexane/t-butyl methyl ether 3:1); ${ }^{1} \mathrm{H}$ NMR $\left(400 \mathrm{MHz}, \mathrm{CDCl}_{3}\right)$ : $\delta=8.71$ (br s, $\left.1 \mathrm{H}\right), 7.34-7.38$ (m, 4 H), 7.19-7.29 (m, 8 H), 6.93 (dd, J=7.7, 0.9 Hz, 1 H), 6.88 (ddd, J=7.1, 7.1, $1.3 \mathrm{~Hz}, 1 \mathrm{H}$ ), 3.55 (br s, 1 $\mathrm{H}), 3.25$ (s, $4 \mathrm{H}$ ) ppm; ${ }^{13} \mathrm{C} \mathrm{NMR}\left(100 \mathrm{MHz}, \mathrm{CDCl}_{3}\right): \delta=156.1,131.8,129.7,128.3,128.3,126.8,126.2$, 122.9, 119.6, 118.0, 84.9, 84.4, 78.2, 32.0 ppm; IR $\left(\mathrm{CHCl}_{3}\right)$ : v=3688, 3367, 3018, 1808, 1699, 1653,

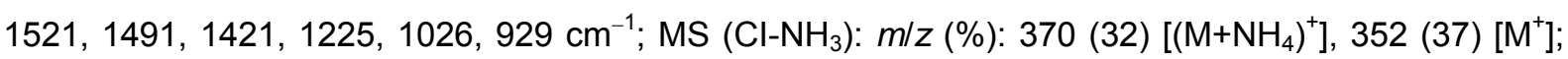
HRMS (EI): $\mathrm{m} / \mathrm{z}$ calcd for $\mathrm{C}_{25} \mathrm{H}_{18} \mathrm{O}\left[\left(\mathrm{M}-\mathrm{H}_{2} \mathrm{O}\right)^{+}\right]: 334.13515$; found: 334.13577 .

4-(2-Bromobenzyl)-1,7-diphenyl-1,6-heptadiyn-4-ol (SI13): In accordance with the protocol described for SI12, two-fold Sonogashira cross-coupling of SI10 (0.500 g, $0.180 \mathrm{mmol})$ with Phl (0.425 $\mathrm{mL}, 0.773 \mathrm{~g}, 3.79 \mathrm{mmol})$ in the presence of $\left(\mathrm{Ph}_{3} \mathrm{P}\right)_{2} \mathrm{PdCl}_{2}(0.013 \mathrm{~g}, 0.018 \mathrm{mmol}, 1.0 \mathrm{~mol} \%)$ and Cul (1.7 mg, $9.0 \mu \mathrm{mol}, 0.50 \mathrm{~mol} \%)$ in $\mathrm{Et}_{3} \mathrm{~N}(10 \mathrm{~mL})$ provided SII3 $(0.739 \mathrm{~g}, 96 \%)$ as a colorless oil. $R_{\mathrm{f}}=0.50$ (cyclohexane/t-butyl methyl ether $4: 1$ ); ${ }^{1} \mathrm{H}$ NMR $\left(500 \mathrm{MHz}, \mathrm{CDCl}_{3}\right): \delta=7.60$ (dd, $J=8.1,1.2 \mathrm{~Hz}$, $1 \mathrm{H}$ ), 7.57 (dd, J=7.7, $1.5 \mathrm{~Hz}, 1 \mathrm{H}$ ), 7.44-7.49 (m, $4 \mathrm{H}$ ), 7.29-7.34 (m, $7 \mathrm{H}$ ), 7.13 (ddd, J=7.7, 7.7, 1.6 $\mathrm{Hz}, 1 \mathrm{H}), 3.44$ (s, $2 \mathrm{H}), 2.91$ (d, J=16.9 Hz, $2 \mathrm{H}), 2.84$ (d, J=16.8 Hz, $2 \mathrm{H}), 2.57$ (s, $1 \mathrm{H}) \mathrm{ppm} ;{ }^{13} \mathrm{C}$ NMR $\left(125 \mathrm{MHz}, \mathrm{CDCl}_{3}\right): \delta=136.6,133.1,132.7,131.8,128.5,128.4,128.1,127.4,126.3,123.3,85.6,84.4$, 74.8, 42.7, 31.0 ppm; IR $\left(\mathrm{CHCl}_{3}\right)$ : v=3563, 3016, 2929, 2245, 1952, 1806, 1750, 1700, 1598, 1490, 1438, 1212, 1066, $926 \mathrm{~cm}^{-1}$; MS $\left(\mathrm{Cl}-\mathrm{NH}_{3}\right): \mathrm{m} / \mathrm{z}(\%): 448(100)\left[\left(\mathrm{M}+\mathrm{NH}_{4}\right)^{+}\right], 446(98)\left[\left(\mathrm{M}+\mathrm{NH}_{4}\right)^{+}\right], 431$ (11) $\left[(\mathrm{M}+\mathrm{H})^{+}\right], 429(10)\left[(\mathrm{M}+\mathrm{H})^{+}\right]$; elemental analysis calcd (\%) for $\mathrm{C}_{26} \mathrm{H}_{21} \mathrm{BrO}$ : C 72.73, H 4.93; found: C 72.42, H 5.27 .

(E,E)-2-[1-Hydroxy-4-phenyl-1-(3-phenyl-2-propenyl)-3-butenyl]phenol [(E,E)-SI14]: At $0^{\circ} \mathrm{C}$, a solution of SI11 $(0.352 \mathrm{~g}, 1.00 \mathrm{mmol})$ and dry THF $(5 \mathrm{~mL})$ was added dropwise to a solution of Red$\mathrm{Al}^{\circledR}(3.5 \mathrm{M}$ in toluene, $2.15 \mathrm{~mL}, 1.52 \mathrm{~g}, 7.53 \mathrm{mmol})$ in dry THF $(5 \mathrm{~mL})$. The cooling bath was removed after $20 \mathrm{~min}$ and the reaction mixture was maintained at room temperature for two days for completion. Then, aq $\mathrm{NaOH}(2.5 \mathrm{M}, 2 \mathrm{~mL})$ was slowly added at $0^{\circ} \mathrm{C}$ to quench the reaction followed by addition of $t$ butyl methyl ether $(100 \mathrm{~mL})$ and $\mathrm{H}_{2} \mathrm{O}(60 \mathrm{~mL})$. The organic layer was washed with brine $(40 \mathrm{~mL})$ and dried over $\mathrm{MgSO}_{4}$. Evaporation of the solvents and purification of the residue by flash column chromatography on silica gel (cyclohexane/t-butyl methyl ether 95:5 $\rightarrow 6: 1)$ furnished $(E, E)$-SI14 $(0.324$ $\mathrm{g}, 91 \%)$ as a colorless oil. The $E, Z$-ratio was determined from the ${ }^{1} \mathrm{H}$ NMR spectra $(E: Z \geq 99: 1) . R_{\mathrm{f}}=0.38$ (cyclohexane/t-butyl methyl ether 3:1); ${ }^{1} \mathrm{H}$ NMR $\left(400 \mathrm{MHz}, \mathrm{CDCl}_{3}\right)$ : $\delta=9.16(\mathrm{~s}, 1 \mathrm{H}), 7.26-7.33(\mathrm{~m}, 8$ H), 7.18-7.25 (m, 3 H), 7.11 (dd, J=7.7, $1.7 \mathrm{~Hz}, 1 \mathrm{H}), 6.85-6.94(\mathrm{~m}, 2 \mathrm{H}), 6.52$ (d, J=15.9 Hz, $2 \mathrm{H}$ ), 
6.14 (ddd, J=15.7, 8.4, 7.1 Hz, 2 H), 2.99 (ddd, J=14.2, 6.5, $1.3 \mathrm{~Hz}, 2 \mathrm{H}$ ), 2.96 (s, $1 \mathrm{H}$ ), 2.76 (dd, $\mathrm{J}=14.2,8.2 \mathrm{~Hz}, 2 \mathrm{H}) \mathrm{ppm} ;{ }^{13} \mathrm{C}$ NMR $\left(100 \mathrm{MHz}, \mathrm{CDCl}_{3}\right): \delta=156.9,136.9,135.3,131.8,129.2,128.6$, 127.7, 126.5, 126.4, 124.0, 119.5, 118.1, 79.7, 44.5 ppm; IR $\left(\mathrm{CHCl}_{3}\right): \mathrm{v}=3529,3330,3156,3034$, 2928, 1795, 1582, 1489, 1382, 1239, 1096, $915 \mathrm{~cm}^{-1}$; MS $\left(\mathrm{Cl}-\mathrm{NH}_{3}\right): \mathrm{m} / \mathrm{z}(\%): 374(52)\left[\left(\mathrm{M}^{\left.\left.-\mathrm{NH}_{4}\right)^{+}\right],} 356\right.\right.$ (34) $\left[\mathrm{M}^{+}\right]$; HRMS (EI): $\mathrm{m} / \mathrm{z}$ calcd for $\mathrm{C}_{25} \mathrm{H}_{20} \mathrm{O}\left[\left(\mathrm{M}-\mathrm{H}_{4} \mathrm{O}\right)^{+}\right]$: 336.15128; found: 336.15142.

$(E, E)-4-(2-B r o m o b e n z y l)-1,7-d i p h e n y l-1,6-h e p t a d i e n-4-o l ~[(E, E)-7]$ : At $0^{\circ} \mathrm{C}$, DIBAL-H (1.0M in $n$ hexane, $12.1 \mathrm{~mL}, 12.1 \mathrm{mmol})$ was diluted with dry THF (3 ml) and a solution of diyne SI13 $(0.260 \mathrm{~g}$, $0.606 \mathrm{mmol}$ ) with dry THF $(3 \mathrm{~mL})$ was added dropwise whereupon $\mathrm{H}_{2}$ was formed. The cooling bath was removed after $10 \mathrm{~min}$ and the reaction mixture was heated to $65^{\circ} \mathrm{C}$ for three days. At $0^{\circ} \mathrm{C}$, the reaction was carefully quenched by slow addition of aq $\mathrm{NaOH}(2.5 \mathrm{M}, 3 \mathrm{~mL})$ followed by dilution with $t$ butyl methyl ether $(100 \mathrm{~mL})$ and aq $\mathrm{HCl}(2 \mathrm{M}, 120 \mathrm{~mL})$. The aqueous layer was back-extracted with $t$ butyl methyl ether $(50 \mathrm{~mL})$ and the combined organic layers were washed with saturated aq $\mathrm{NaHCO}_{3}$ $(50 \mathrm{~mL})$ and dried $\left(\mathrm{MgSO}_{4}\right)$. Evaporation of the solvents under reduced pressure and purification of the residue by flash column chromatography on silica gel (cyclohexane/t-butyl methyl ether $97: 3 \rightarrow 6: 1$ ) provided the desired diene $(E, E)-7(0.251 \mathrm{~g}, 95 \%)$ as a colorless oil. The $E, Z$-ratio was determined from the ${ }^{1} \mathrm{H}$ NMR spectra $(E: Z \geq 99: 1) . R_{\mathrm{f}}=0.36$ (cyclohexane/t-butyl methyl ether $\left.4: 1\right) ;{ }^{1} \mathrm{H}$ NMR $(400$ $\left.\mathrm{MHz}, \mathrm{CDCl}_{3}\right): \delta=7.59$ (dd, J=8.0, $\left.1.3 \mathrm{~Hz}, 1 \mathrm{H}\right), 7.45$ (dd, J=7.6, $\left.1.7 \mathrm{~Hz}, 1 \mathrm{H}\right), 7.35-7.38(\mathrm{~m}, 4 \mathrm{H})$, 7.29-7.34 (m, 4 H), 7.20-7.28 (m, 3 H), 7.11 (ddd, J=8.0, 7.3, $1.8 \mathrm{~Hz}, 1 \mathrm{H}$ ), 6.49 (d, J= $15.8 \mathrm{~Hz}, 2 \mathrm{H}$ ), 6.32 (ddd, J=15.9, 7.4, 7.4 Hz, $2 \mathrm{H}$ ), 3.15 (s, $2 \mathrm{H}$ ), 2.56 (ddd, J=14.0, 7.5, $1.2 \mathrm{~Hz}, 2 \mathrm{H}$ ), 2.46 (ddd, $\mathrm{J}=14.0,7.2,1.3 \mathrm{~Hz}, 2 \mathrm{H}), 1.91(\mathrm{~s}, 1 \mathrm{H}) \mathrm{ppm} ;{ }^{13} \mathrm{C} \mathrm{NMR}\left(100 \mathrm{MHz}, \mathrm{CDCl}_{3}\right): \delta=137.4,137.3,134.1$, 133.1 , 132.7, 128.6, 128.3, 127.4, 127.3, 126.3, 126.2, 125.3, 75.4, 44.7, 43.4 ppm; IR $\left(\mathrm{CDCl}_{3}\right)$ : $\mathrm{v}=3574,3063,3029,2929,1950,1598,1496,1439,1368,1026,970 \mathrm{~cm}^{-1} ; \mathrm{MS}\left(\mathrm{Cl}_{-} \mathrm{NH}_{3}\right): \mathrm{m} / \mathrm{z}(\%): 452$ (97) $\left[\left(\mathrm{M}+\mathrm{NH}_{4}\right)^{+}\right], 450(100)\left[\left(\mathrm{M}+\mathrm{NH}_{4}\right)^{+}\right]$; elemental analysis calcd (\%) for $\mathrm{C}_{26} \mathrm{H}_{25} \mathrm{BrO}$ : C 72.06, H 5.81; found: C 71.98, H 6.10 .

$(E, E)$-Trifluoromethanesulfonic acid 2-[1-hydroxy-4-phenyl-1-(3-phenyl-2-propenyl)-3pentenyl]phenyl ester $[(E, E)-8]$ : At $-30^{\circ} \mathrm{C}, 2,6$-lutidine $(0.054 \mathrm{~mL}, 0.050 \mathrm{~g}, 0.47 \mathrm{mmol})$, DMAP (8.0 $\mathrm{mg}, 0.070 \mathrm{mmol}, 22 \mathrm{~mol} \%)$ and $\mathrm{Tf}_{2} \mathrm{O}(0.078 \mathrm{~mL}, 0.13 \mathrm{~g}, 0.47 \mathrm{mmol})$ were successively added to a solution of $(E, E)-\mathrm{SI} 14(0.110 \mathrm{~g}, 0.310 \mathrm{mmol})$ in dry $\mathrm{CH}_{2} \mathrm{Cl}_{2}(2.5 \mathrm{~mL})$. The cooling bath was removed after $20 \mathrm{~min}$ and the reaction mixture was allowed to warm to room temperature for $3 \mathrm{~h}$. The reaction was quenched adding $\mathrm{H}_{2} \mathrm{O}(30 \mathrm{~mL})$, extracted with $t$-butyl methyl ether $(2 \times 30 \mathrm{~mL})$ and dried $\left(\mathrm{Na}_{2} \mathrm{SO}_{4}\right)$. Evaporation of the volatiles under reduced pressure and purification of the residue by flash column chromatography on silica gel (cyclohexane/t-butyl methyl ether $9: 1)$ provided $(E, E)-8(0.130 \mathrm{~g}$, $87 \%$ ) as a colorless oil. $R_{\mathrm{f}}=0.46$ (cyclohexane/t-butyl methyl ether $\left.3: 1\right) ;{ }^{1} \mathrm{H}$ NMR $\left(500 \mathrm{MHz}, \mathrm{CDCl}_{3}\right)$ : $\delta=7.66-7.69(\mathrm{~m}, 1 \mathrm{H}), 7.33-7.37(\mathrm{~m} 3 \mathrm{H}), 7.25-7.29(\mathrm{~m}, 8 \mathrm{H}), 7.21\left(\mathrm{~m}_{\mathrm{c}}, 2 \mathrm{H}\right), 6.49(\mathrm{~d}, \mathrm{~J}=15.9 \mathrm{~Hz}, 2 \mathrm{H})$, 6.03 (ddd, J=15.6, 8.4, 7.0 Hz, 2 H), 3.07 (ddd, J=14.4, 6.7, $1.5 \mathrm{~Hz}, 2 \mathrm{H}$ ), 2.82 (ddd, J=14.2, 8.2, 1.2 $\mathrm{Hz}, 2 \mathrm{H}), 2.51$ (s, $1 \mathrm{H}) \mathrm{ppm} ;{ }^{13} \mathrm{C} \mathrm{NMR}\left(125 \mathrm{MHz}, \mathrm{CDCl}_{3}\right)$ : $\delta=147.0,137.4,137.1,134.9,129.7,129.4$, 128.6, 128.1, 127.6, 126.3, 124.1, 121.5, 118.6 (q, J(C,F)=320 Hz), 75.7, 44.4 ppm; IR $\left(\mathrm{CHCl}_{3}\right)$ : $\mathrm{v}=3687,3021,1806,1700,1651,1521,1421,1233,1142,1074,970,928,881 \mathrm{~cm}^{-1} ; \mathrm{MS}\left(\mathrm{Cl}-\mathrm{NH}_{3}\right)$ : 
m/z (\%): $506(100)\left[\left(\mathrm{M}+\mathrm{NH}_{4}\right)^{+}\right]$; elemental analysis calcd (\%) for $\mathrm{C}_{26} \mathrm{H}_{23} \mathrm{~F}_{3} \mathrm{O}_{4} \mathrm{~S}: \mathrm{C}$ 63.92, H 4.75, S 6.56; found: C 64.17, H 4.45, S 6.44 .

$(E, E)$-Trifluoromethanesulfonic acid 2-[5-phenyl-2-(3-phenyl-2-propenyl)-2-triethylsilyloxy-4pentenyl]phenyl ester $[(E, E)-10]$ : At $0^{\circ} \mathrm{C}, 2,6$-lutidine $(0.188 \mathrm{~mL}, 0.173 \mathrm{~g}, 1.61 \mathrm{mmol})$ and $\mathrm{Et}_{3} \mathrm{SiOTf}$ $(0.164 \mathrm{~mL}, 0.189 \mathrm{~g}, 0.716 \mathrm{mmol})$ were subsequently added via syringe to a solution of $(E, E)-9(0.180$ $\mathrm{g}, 0.358 \mathrm{mmol})$ in dry $\mathrm{CH}_{2} \mathrm{Cl}_{2}(10 \mathrm{~mL})$. After $30 \mathrm{~min}$, the cooling bath was removed and the reaction mixture was maintained for $2 \mathrm{~h}$ at ambient temperature. Dilution with $t$-butyl methyl ether $(60 \mathrm{~mL})$ was followed by washing with aq $\mathrm{HCL}(2 \mathrm{M}, 20 \mathrm{~mL})$ and saturated aq $\mathrm{NaHCO}_{3}(20 \mathrm{~mL})$. The organic layer was dried over anhydrous $\mathrm{Na}_{2} \mathrm{SO}_{4}$, evaporated and the residue was purified by flash column chromatography on silica gel (cyclohexane/t-butyl methyl ether 100:1) affording $(E, E)-10(0.112 \mathrm{~g}$, $51 \%$ ) as a colorless oil. $R_{\mathrm{f}}=0.76$ (cyclohexane/t-butyl methyl ether $\left.9: 1\right) ;{ }^{1} \mathrm{H}$ NMR $\left(400 \mathrm{MHz}, \mathrm{CDCl}_{3}\right)$ : $\delta=7.55-7.61(\mathrm{~m}, 1 \mathrm{H}), 7.17-7.38(\mathrm{~m}, 13 \mathrm{H}), 6.51$ (d, J=15.9 Hz, $2 \mathrm{H}$ ), 6.26 (ddd, J=15.8, 7.0, 7.0 Hz, 2 $\mathrm{H}), 3.02(\mathrm{~s}, 2 \mathrm{H}), 2.49\left(\mathrm{~m}_{\mathrm{c}}, 4 \mathrm{H}\right), 0.95(\mathrm{t}, \mathrm{J}=7.9 \mathrm{~Hz}, 9 \mathrm{H}), 0.65$ (q, J=7.9 Hz, $\left.6 \mathrm{H}\right) \mathrm{ppm} ;{ }^{13} \mathrm{C}$ NMR $(100$ $\left.\mathrm{MHz}, \mathrm{CDCl}_{3}\right): \delta=149.1,137.6,134.1,133.5,131.2,128.6,128.3,127.6,127.3,126.2,125.6,121.1$, 118.7 (q, J(C,F)=320 Hz), 78.5, 44.3, 39.2, 7.3, 7.1 ppm; IR $\left(\mathrm{CDCl}_{3}\right): \mathrm{v}=3692,3030,2958,2879,1950$, 1803, 1599, 1491, 1419, 1219, 1142, 1010, $969 \mathrm{~cm}^{-1}$; MS $\left(\mathrm{Cl}-\mathrm{NH}_{3}\right): \mathrm{m} / \mathrm{z}(\%): 634$ (16) [(M+NH$\left.)^{+}\right]$; HRMS (EI): $m / z$ calcd for $\mathrm{C}_{24} \mathrm{H}_{30} \mathrm{~F}_{3} \mathrm{O}_{4} \mathrm{SSi}\left[\left(\mathrm{M}-\mathrm{C}_{9} \mathrm{H}_{9}\right)^{+}\right]$: 499.16012; found: 499.15861.

(E,E)-1-(2-Hydroxyphenyl)-5-phenyl-2-(3-phenyl-2-propenyl)-4-pentene-1-one $\quad[(E, E)-S I 17]: \quad$ At room temperature, $\mathrm{Pd}(\mathrm{OAc})_{2}(0.011 \mathrm{~g}, 0.050 \mathrm{mmol}, 5.0 \mathrm{~mol} \%), \mathrm{Ph}_{3} \mathrm{P}(0.026 \mathrm{~g}, 0.10 \mathrm{mmol}, 10 \mathrm{~mol} \%)$ and cinnamic alcohol $(0.322 \mathrm{~g}, 2.40 \mathrm{mmol})$ were added to a Schlenk flask and dissolved in degassed dry THF (5 mL). To the solution were subsequently added 2-hydroxyacetophenone (SI16) $(1.20 \mathrm{~mL}$, $0.136 \mathrm{~g}, 1.00 \mathrm{mmol})$ and $\mathrm{Et}_{3} \mathrm{~B}(1.0 \mathrm{M}$ in $n$-hexane, $2.40 \mathrm{~mL}, 2.40 \mathrm{mmol})$ and the resulting reaction mixture was heated at $50^{\circ} \mathrm{C}$ for $6 \mathrm{~h}$. At room temperature, $t$-butyl methyl ether $(100 \mathrm{~mL})$ was added and the mixture was washed with $\mathrm{H}_{2} \mathrm{O}(2 \times 60 \mathrm{~mL})$ and dried $\left(\mathrm{Na}_{2} \mathrm{SO}_{4}\right)$. Evaporation of the solvents in vacuo and purification of the residue by flash column chromatography on silica gel (cyclohexane/tbutyl methyl ether $97: 3)$ afforded $(E, E)$-SI17 $(0.266 \mathrm{~g}, 80 \%)$ as a colorless oil. The $E, Z$-ratio was determined from the ${ }^{1} \mathrm{H}$ NMR spectra $(E, Z>99: 1) . R_{\mathrm{f}}=0.75$ (cyclohexane/t-butyl methyl ether $3: 1$ ); ${ }^{1} \mathrm{H}$ NMR $\left(500 \mathrm{MHz}, \mathrm{CDCl}_{3}\right.$ ): $\delta=12.54$ (s, $1 \mathrm{H}$ ), 7.81 (dd, J=8.2, 1.7, $1 \mathrm{H}$ ), 7.46 (ddd, J=8.5, 7.2, $1.5 \mathrm{~Hz}, 1$ H), 7.24-7.30 (m, $8 \mathrm{H}), 7.16-7.21(\mathrm{~m}, 2 \mathrm{H}), 7.00$ (dd, J=8.4, $1.2 \mathrm{~Hz}, 1 \mathrm{H}), 6.90$ (ddd, J=8.1, 7.2, 1.1 $\mathrm{Hz}, 1 \mathrm{H}$ ), 6.44 (d, J=15.8 Hz, $2 \mathrm{H}$ ), 6.15 (ddd, J=15.8, 7.3, 7.3 Hz, $2 \mathrm{H}$ ), 3.76 (tt, J=6.7, $6.7 \mathrm{~Hz}, 1 \mathrm{H}$ ), 2.73 (dddd, J=14.2, 7.1, 7.1, $1.4 \mathrm{~Hz}, 2 \mathrm{H}$ ), 2.53 (dddd, $\mathrm{J}=14.2,7.7,6.4,1.3 \mathrm{~Hz}, 2 \mathrm{H}$ ) ppm; ${ }^{13} \mathrm{C}$ NMR $\left(125 \mathrm{MHz}, \mathrm{CDCl}_{3}\right): \delta=208.7,163.2,137.2,136.6,134.2,132.7,129.9,128.6,127.4,126.7,126.2$, 119.0, 118.9, 46.1, 35.4 ppm; IR (neat): v=3026, 2931, 2948, 1948, 1877, 1804, 1634, 1488, 1446,

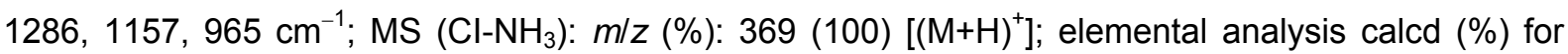
$\mathrm{C}_{26} \mathrm{H}_{24} \mathrm{O}_{2}: \mathrm{C} 84.75$, H 6.57; found: C 84.56, H 6.60.

$(E, E)-2-[1-H y d r o x y-5-p h e n y l-2-(3-p h e n y l-2-p r o p e n y l)-4-p e n t e n y l] p h e n o l ~[(E, E)-S I 18]:$ At $0^{\circ} C$, to a suspension of $\mathrm{LiAlH}_{4}(0.114 \mathrm{~g}, 3.00 \mathrm{mmol})$ in dry THF $(8 \mathrm{~mL})$ was added dropwise a solution of ketone $(E, E)$-SI17 $(0.368 \mathrm{~g}, 1.00 \mathrm{mmol})$ and dry THF $(2 \mathrm{~mL})$. The ice bath was removed after $15 \mathrm{~min}$ and the 
resulting reaction mixture was maintained at room temperature for $6 \mathrm{~h}$. The reaction was quenched by slow addition of aq $\mathrm{NaOH}(2.5 \mathrm{M}, 2.0 \mathrm{~mL})$ at $0^{\circ} \mathrm{C}$, then diluted with $t$-butyl methyl ether $(100 \mathrm{~mL})$, washed with $\mathrm{H}_{2} \mathrm{O}(50 \mathrm{~mL})$ and brine $(50 \mathrm{~mL})$ and dried over $\mathrm{Na}_{2} \mathrm{SO}_{4}$. The solvents were evaporated under reduced pressure and the residue was purified by flash column chromatography on silica gel (cyclohexane/t-butyl methyl ether 9:1) affording the diol $(E, E)$-SI18 $(0.270 \mathrm{~g}, 73 \%)$ as a colorless oil. $R_{\mathrm{f}}=0.42$ (cyclohexane/t-butyl methyl ether $3: 1$ ); ${ }^{1} \mathrm{H}$ NMR $\left(500 \mathrm{MHz}, \mathrm{CDCl}_{3}\right): \delta=7.99(\mathrm{~s}, 1 \mathrm{H}), 7.29-7.37$ (m, 8 H), 7.21-7.25 (m, 2 H), 7.18 (ddd, J=7.7, 7.7, 1.7 Hz, 1 H), 6.97 (dd, J=7.7, 1.7 Hz, 1 H), 6.89 (dd, J=8.2, $1.2 \mathrm{~Hz}, 1 \mathrm{H}$ ), 6.86 (ddd, J=7.4, 7.4, $1.2 \mathrm{~Hz}, 1 \mathrm{H}$ ), 6.48 (d, J=15.8 Hz, $1 \mathrm{H}$ ), 6.40 (d, J=15.7 $\mathrm{Hz}, 1 \mathrm{H}$ ), 6.24 (ddd, J=15.7, 7.9, $6.9 \mathrm{~Hz}, 1 \mathrm{H}$ ), 6.15 (ddd, J=15.7, 7.1, $7.1 \mathrm{~Hz}, 1 \mathrm{H}$ ), 4.90 (dd, J=5.6, 3.9 $\mathrm{Hz}, 1 \mathrm{H}), 2.78(\mathrm{~d}, \mathrm{~J}=2.8 \mathrm{~Hz}, 1 \mathrm{H}), 2.46-2.58(\mathrm{~m}, 2 \mathrm{H}), 2.19-2.30(\mathrm{~m}, 3 \mathrm{H}) \mathrm{ppm} ;{ }^{13} \mathrm{C}$ NMR $(125 \mathrm{MHz}$, $\left.\mathrm{CDCl}_{3}\right): \delta=156.0,137.6,137.5,132.3,132.2,129.1,128.6,128.3,128.3,128.1,127.2,127.2,126.1$, 126.1, 125.9, 119.8, 117.4, 78.2, 44.9, 33.9, 32.4 ppm; IR $\left(\mathrm{CHCl}_{3}\right): \mathrm{v}=3597,3367,3010,2939,1944$, 1805, 1699, 1587, 1491, 1437, 1367, 1242, 1076, 967, $930 \mathrm{~cm}^{-1}$; MS (EI): $\mathrm{mlz}(\%): 370$ (2) [M+], 352 (37) [(M- $\left.\left.\mathrm{H}_{2} \mathrm{O}\right)^{+}\right]$; HRMS (EI): $\mathrm{m} / \mathrm{z}$ calcd for $\mathrm{C}_{26} \mathrm{H}_{24} \mathrm{O}\left[\left(\mathrm{M}-\mathrm{H}_{2} \mathrm{O}\right)^{+}\right]: 352.18223$; found: 352.18272.

$(E, E)-2-[5-P h e n y l-2-(3-p h e n y l-2-p r o p e n y l)-4-p e n t e n y l] p h e n o l ~[(E, E)-S I 19]:$ At $0^{\circ} \mathrm{C}$, to a solution of diol $(E, E)$-SI18 $(0.260 \mathrm{~g}, 0.702 \mathrm{mmol})$ and dry $\mathrm{Et}_{2} \mathrm{O}(7 \mathrm{~mL})$ were successevely added $\mathrm{Et}_{3} \mathrm{~N}(0.237 \mathrm{~mL}$, $0.170 \mathrm{~g}, 1.68 \mathrm{mmol})$ and methanesulfonyl chloride $(0.131 \mathrm{~mL}, 0.193 \mathrm{~g}, 1.68 \mathrm{mmol})$. After $3.5 \mathrm{~h}$ at this temperature, $\mathrm{LiAlH}_{4}(0.200 \mathrm{~g}, 5.26 \mathrm{mmol})$ was carefully added, the cooling bath was removed after 10 $\mathrm{min}$, and the reaction mixture was maintained at ambient temperature overnight. The reaction was quenched at $0^{\circ} \mathrm{C}$ by slow addition of aq $\mathrm{NaOH}(2.5 \mathrm{M}, 1.5 \mathrm{~mL})$, then diluted with $t$-butyl methyl ether (80 $\mathrm{mL}$ ) and washed with $\mathrm{H}_{2} \mathrm{O}(40 \mathrm{~mL})$ and brine $(40 \mathrm{~mL})$. The organic phase was dried $\left(\mathrm{Na}_{2} \mathrm{SO}_{4}\right)$, evaporated and the residue was dissolved in $\mathrm{MeOH}(20 \mathrm{~mL})$ and aq $\mathrm{NaOH}(1 \mathrm{M}, 0.1 \mathrm{~mL})$ and heated to $50^{\circ} \mathrm{C}$ for $2 \mathrm{~h}$. After drying over $\mathrm{MgSO}_{4}$ and evaporation of the volatiles, the resulting residue was purified by flash column chromatography on silica gel (cyclohexane/t-butyl methyl ether 98:2) to afford $(E, E)$-SI19 $(0.098 \mathrm{~g}, 39 \%)$ as a colorless oil. $R_{\mathrm{f}}=0.33$ (cyclohexane/t-butyl methyl ether $\left.3: 1\right) ;{ }^{1} \mathrm{H}$ NMR (400 MHz, $\mathrm{CDCl}_{3}$ ): $\delta=7.26-7.35(\mathrm{~m}, 8 \mathrm{H}), 7.18\left(\mathrm{~m}_{\mathrm{c}}, 2 \mathrm{H}\right), 7.12(\mathrm{dd}, \mathrm{J}=7.3,1.7 \mathrm{~Hz}, 1 \mathrm{H}), 7.07$ (ddd, $\mathrm{J}=7.7,7.7,1.7 \mathrm{~Hz}, 1 \mathrm{H}), 6.86$ (ddd, J=7.1, 7.1, $1.3 \mathrm{~Hz}, 1 \mathrm{H}$ ), 6.73 (d, J=7.7 Hz, $1 \mathrm{H}$ ), 6.42 (d, J=15.9 $\mathrm{Hz}, 2 \mathrm{H}$ ), 6.25 (ddd, J=15.5, 7.5, 7.5 Hz, $2 \mathrm{H}$ ), 5.17 (s, $1 \mathrm{H}$ ), 2.66 (d, J=7.3 Hz, $2 \mathrm{H}$ ), 2.27 (mc, $2 \mathrm{H}$ ), $2.07\left(\mathrm{~m}_{\mathrm{c}}, 1 \mathrm{H}\right) \mathrm{ppm} ;{ }^{13} \mathrm{C} \mathrm{NMR}\left(100 \mathrm{MHz}, \mathrm{CDCl}_{3}\right): \delta=154.0,137.9,131.8,131.4,129.1,128.6,127.3$, 127.0, 126.1, 125.9, 120.6, 115.4, 39.3, 37.1, 34.4 ppm; IR $\left(\mathrm{CHCl}_{3}\right): \mathrm{v}=3596,3009,2980,2921,1942$, 1805, 1648, 1590, 1492, 1453, 1228, 1071, $964 \mathrm{~cm}^{-1}$; MS (EI): m/z (\%): 354 (18) [M+']; HRMS (EI): m/z calcd for $\mathrm{C}_{26} \mathrm{H}_{26} \mathrm{O}\left[\mathrm{M}^{+}\right]$: 354.19769; found: 354.19837.

$(E, E)$-Trifluoromethanesulfonic acid 2-[5-phenyl-2-(3-phenyl-2-propenyl)-4-pentenyl]phenyl ester [(E,E)-12]: According to the preparation of $(E, E)-9$, reaction of $(E, E)$-SI19 $(0.270 \mathrm{~g}, 0.762 \mathrm{mmol})$ with $\mathrm{PhNTf}_{2}(0.272 \mathrm{~g}, 0.762 \mathrm{mmol})$ and $\mathrm{Cs}_{2} \mathrm{CO}_{3}(0.248 \mathrm{~g}, 0.762 \mathrm{mmol})$ in dry DMF $(7 \mathrm{~mL})$ afforded desired $(E, E)-12(0.275 \mathrm{~g}, 74 \%)$ as a colorless oil. $R_{\mathrm{f}}=0.14$ (cyclohexane); ${ }^{1} \mathrm{H}$ NMR $\left(500 \mathrm{MHz}, \mathrm{CDCl}_{3}\right)$ : $\delta=7.28-7.38$ (m, $12 \mathrm{H}$ ), 7.21-7.25 (tt, J=7.2, $1.5 \mathrm{~Hz}, 2 \mathrm{H}$ ), 6.41 (d, J=15.7 Hz, $2 \mathrm{H}$ ), 6.20 (ddd, J=15.8, 7.3, 7.3 Hz, $2 \mathrm{H}), 2.81(\mathrm{~d}, \mathrm{~J}=7.2 \mathrm{~Hz}, 2 \mathrm{H}), 2.29\left(\mathrm{~m}_{\mathrm{c}}, 4 \mathrm{H}\right), 2.13\left(\mathrm{~m}_{\mathrm{c}}, 1 \mathrm{H}\right) \mathrm{ppm} ;{ }^{13} \mathrm{C} N M R(125 \mathrm{MHz}$, $\mathrm{CDCl}_{3}$ ): $\delta=148.6,137.6,134.0,132.3,132.2,128.6,128.3,128.1,127.1,126.1,121.5,118.7$ (q, 
$J(C, F)=320 \mathrm{~Hz}), 39.4,37.0,34.9 \mathrm{ppm}$; IR $\left(\mathrm{CHCl}_{3}\right): \mathrm{v}=3677,3034,2923,1945,1808,1653,1599$,

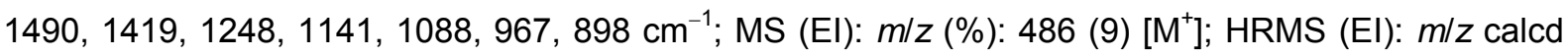
for $\mathrm{C}_{27} \mathrm{H}_{25} \mathrm{~F}_{3} \mathrm{O}_{4} \mathrm{~S}\left[\mathrm{M}^{+}\right]$: 486.14689 ; found: 486.14764 .

\section{Trifluoromethanesulfonic}

acid

2-[2-hydroxy-5-phenyl-2-(3-phenyl-2-propynyl)-4pentynyl]phenyl ester (SI20): According to the procedure described for the preparation of $(E, E)-\mathbf{9}$, reaction of SII2 $(0.411 \mathrm{~g}, 1.20 \mathrm{mmol})$ with $\mathrm{PhNTf}_{2}(0.430 \mathrm{~g}, 1.20 \mathrm{mmol})$ and $\mathrm{Cs}_{2} \mathrm{CO}_{3}(0.392 \mathrm{~g}, 1.20$ $\mathrm{mmol})$ in dry DMF $(10 \mathrm{~mL})$ afforded desired $\mathrm{SI} 20(0.532 \mathrm{~g}, 89 \%)$ as a colorless oil. $R_{\mathrm{f}}=0.44$ (cyclohexane/t-butyl methyl ether 3:1); ${ }^{1} \mathrm{H}$ NMR $\left(400 \mathrm{MHz}, \mathrm{CDCl}_{3}\right)$ : $\delta=7.67\left(\mathrm{~m}_{\mathrm{c}}, 1 \mathrm{H}\right), 7.44-7.49(\mathrm{~m}, 4$ H), 7.35-7.39 (m, 2 H), 7.30-7.35 (m, 7 H), 3.29 (s, 2 H), 2.86 (d, J=16.8 Hz, 2 H), 2.82 (d, J=16.8 Hz, $2 \mathrm{H}$ ), 2.45 (br s, $1 \mathrm{H}$ ) ppm; ${ }^{13} \mathrm{C}-\mathrm{NMR}\left(100 \mathrm{MHz}, \mathrm{CDCl}_{3}\right): \delta=149.1,133.7,131.8,129.8,128.9,128.3$, 128.2, 128.1, 123.1, 121.4, 118.6 (q, J(C,F)=320 Hz), 84.8, 84.8, 74.1, 38.0, 31.0 ppm; IR $\left(\mathrm{CDCl}_{3}\right)$ : $\mathrm{v}=3562,3065,2929,2254,1952,1882,1599,1490,1420,1220,1140,1091 \mathrm{~cm}^{-1} ; \mathrm{MS}\left(\mathrm{Cl}_{-} \mathrm{NH}_{3}\right): \mathrm{m} / \mathrm{z}$ (\%): $516(100)\left[\left(\mathrm{M}+\mathrm{NH}_{4}\right)^{+}\right], 498(4)\left[\mathrm{M}^{+}\right]$; HRMS (El): $\mathrm{m} / \mathrm{z}$ calcd for $\mathrm{C}_{27} \mathrm{H}_{21} \mathrm{~F}_{3} \mathrm{O}_{4} \mathrm{~S}\left[\mathrm{M}^{+}\right]: 498.11150$; found: 498.11126 .

\section{(Z,Z)-Trifluoromethanesulfonic acid 2-[2-hydroxy-5-phenyl-2-(3-phenyl-2-propenyl)-4-} pentenyl]phenyl ester [(Z,Z)-9]: At room temperature, a Schlenk flask charged with $\mathrm{Pd} / \mathrm{CaCO}_{3}(5 \%$ $\mathrm{Pd}, 0.026 \mathrm{~g}, 5.0 \%$ ) was evacuated for $5 \mathrm{~min}$ and backfilled with $\mathrm{H}_{2}$ (balloon) for $10 \mathrm{~min}$ (three cycles) in order to activate the catalyst. A solution of diyne $S I 20(0.520 \mathrm{~g}, 1.04 \mathrm{mmol})$, freshly distilled quinoline $(0.30 \mathrm{~mL}, 0.32 \mathrm{~g}, 2.5 \mathrm{mmol})$ and dry EtOH $(10 \mathrm{~mL})$ was added and the resulting suspension was vigorously stirred under $\mathrm{H}_{2}$ atmosphere overnight. The reaction mixture was filtered through a pad of celite, diluted with $t$-butyl methyl ether $(80 \mathrm{~mL})$, and successively washed with aq $\mathrm{HCl}(2 \mathrm{M}, 2 \times 30$ $\mathrm{mL}$ ) and saturated aq $\mathrm{NaHCO}_{3}(30 \mathrm{~mL})$. Drying over $\mathrm{MgSO}_{4}$ followed by evaporation of the solvents and purification of the resulting residue by flash column chromatography (cyclohexane/t-butyl methyl ether 9:1) provided $(Z, Z)-9(0.466 \mathrm{~g}, 89 \%)$ as a colorless oil. The $Z, E$-ratio was determined from the ${ }^{1} \mathrm{H}$ NMR spectra ( $Z: E>99: 1) . R_{\mathrm{f}}=0.41$ (cyclohexane/t-butyl methyl ether $3: 1$ ); ${ }^{1} \mathrm{H}$ NMR $\left(400 \mathrm{MHz}, \mathrm{CDCl}_{3}\right.$ ): $\delta=7.27-7.33(\mathrm{~m}, 5 \mathrm{H}), 7.20-7.28(\mathrm{~m}, 8 \mathrm{H}), 7.13\left(\mathrm{~m}_{\mathrm{c}}, 1 \mathrm{H}\right), 6.62(\mathrm{~d}, \mathrm{~J}=11.7 \mathrm{~Hz}, 2 \mathrm{H}), 5.73$ (ddd, J=11.9, 7.4, $7.4 \mathrm{~Hz}, 2 \mathrm{H}$ ), 2.91 (s, $2 \mathrm{H}$ ), 2.61 (ddd, J=15.1, 7.5, $1.8 \mathrm{~Hz}, 2 \mathrm{H}$ ), 2.54 (ddd, J=15.1, 7.3, $1.9 \mathrm{~Hz}, 2$ $\mathrm{H}), 1.61(\mathrm{~s}, 1 \mathrm{H}) \mathrm{ppm} ;{ }^{13} \mathrm{C}$ NMR $\left(100 \mathrm{MHz}, \mathrm{CDCl}_{3}\right): \delta=148.9,137.1,133.8,132.7,130.1,128.8,128.5$, 128.3, 127.9, 127.0, 126.1, 121.3, 118.6 (q, J(C,F)=320 Hz), 75.2, 39.1, 37.6 ppm; IR $\left(\mathrm{CDCl}_{3}\right)$ : $v=3584,3025,2929,1954,1759,1600,1493,1419,1220,1140,910 \mathrm{~cm}^{-1} ; \mathrm{MS}\left(\mathrm{Cl}_{-} \mathrm{NH}_{3}\right): \mathrm{m} / \mathrm{z}(\%): 520$ (100) $\left[\left(\mathrm{M}+\mathrm{NH}_{4}\right)^{+}\right]$; elemental analysis calcd (\%) for $\mathrm{C}_{27} \mathrm{H}_{25} \mathrm{~F}_{3} \mathrm{O}_{4} \mathrm{~S}$ : C $64.53, \mathrm{H} \mathrm{5.01}$; found: C $64.35, \mathrm{H}$ 4.98 .

(Z,Z)-Trifluoromethanesulfonic acid 2-[2-methoxy-5-phenyl-2-(3-phenyl-2-propenyl)-4pentenyl]phenyl ester $[(Z, Z)-11]$ : According to the preparation of $(E, E)-11$, reaction of alcohol $(Z, Z)-\mathbf{9}$ $(0.200 \mathrm{~g}, 0.398 \mathrm{mmol})$ with trimethyloxonium tetrafluoro borate $(0.295 \mathrm{~g}, 1.99 \mathrm{mmol})$ and $1,8-$ bis(dimethylamino)naphthalene $(0.430 \mathrm{~g}, 0.201 \mathrm{mmol})$ in dry $\mathrm{CH}_{2} \mathrm{Cl}_{2}(9 \mathrm{~mL})$ afforded $(Z, Z)-11(0.104 \mathrm{~g}$, $50 \%)$ along with unconsumed $(Z, Z)-9(0.082 \mathrm{~g}, 41 \%)$ as colorless oils. $R_{\mathrm{f}}=0.57$ (cyclohexane/t-butyl methyl ether 9:1); ${ }^{1} \mathrm{H}$ NMR (400 MHz, $\left.\mathrm{CDCl}_{3}\right)$ : $\delta=7.29-7.35(\mathrm{~m}, 4 \mathrm{H}), 7.21-7.26(\mathrm{~m}, 6 \mathrm{H}), 7.12-7.20$ 
( $m, 3 \mathrm{H}), 6.98\left(\mathrm{~m}_{\mathrm{c}}, 1 \mathrm{H}\right), 6.53$ (ddd, J=11.9, 1.9, $\left.1.9 \mathrm{~Hz}, 2 \mathrm{H}\right), 5.74$ (ddd, J=11.8, 6.8, $6.8 \mathrm{~Hz}, 2 \mathrm{H}$ ), 3.24 $(\mathrm{s}, 3 \mathrm{H}), 2.91$ (s, $2 \mathrm{H}), 2.57\left(\mathrm{~m}_{\mathrm{c}}, 4 \mathrm{H}\right) \mathrm{ppm} ;{ }^{13} \mathrm{C} \mathrm{NMR}\left(100 \mathrm{MHz}, \mathrm{CDCl}_{3}\right): \delta=148.8,137.4,133.2,131.2$, 130.4, 128.8, 128.2, 128.1, 126.8, 126.5, 121.0, 118.6 (q, J(C,F)=320 Hz), 79.5, 49.3, 34.2, 33.6 ppm; IR $\left(\mathrm{CDCl}_{3}\right): \mathrm{v}=3026,2938,1600,1492,1419,1219,1141,1093 \mathrm{~cm}^{-1} ; \mathrm{MS}\left(\mathrm{Cl}-\mathrm{NH}_{3}\right): \mathrm{m} / \mathrm{z}(\%): 534(100)$ [(M+NH$\left.\left.)_{4}\right)^{+}\right]$; HRMS (EI): $\mathrm{m} / z$ calcd for $\mathrm{C}_{19} \mathrm{H}_{18} \mathrm{~F}_{3} \mathrm{O}_{4} \mathrm{~S}\left[\left(\mathrm{M}-\mathrm{C}_{9} \mathrm{H}_{9}\right)^{+}\right]: 399.08810$; found: 399.08778 .

\section{$(R, E, E)$-4-Benzylidene-2-(3-phenyl-2-propenyl)-2-triethylsilyloxy-1,2,3,4-tetrahydronaphthalene}

[(R,E,E)-14]: In analogy to the procedure described for $(R, E, E)-13$, Heck reaction of $(E, E)-10(0.049 \mathrm{~g}$, $0.079 \mathrm{mmol}$ ) with $\mathrm{Pd}(\mathrm{OAc})_{2}(0.89 \mathrm{mg}, 0.0039 \mathrm{mmol}, 5.0 \mathrm{~mol} \%$ ), (R)-BINAP (3.7 mg, $0.0059 \mathrm{mmol}, 7.5$ mol\%) and $\mathrm{K}_{2} \mathrm{CO}_{3}(0.044 \mathrm{~g}, 0.32 \mathrm{mmol})$ in toluene $(0.79 \mathrm{~mL})$ at $80^{\circ} \mathrm{C}$ for $15 \mathrm{~h}$ provided after purification by flash column chromatography on silica gel (cyclohexane/t-butyl methyl ether 95:5) $(R, E, E)-14(0.020 \mathrm{~g}, 55 \%)$ as a colorless oil. The enantiomeric excess was determined by HPLC analysis $\left(2 \%\right.$ ee). As determined from the ${ }^{1} \mathrm{H}$ NMR spectra, $(R, E, E)-14$ was isomerically pure. $R_{\mathrm{f}}=0.83$ (cyclohexane/t-butyl methyl ether 9:1); HPLC (Daicel Chiracel AD, $n$-heptane/i-PrOH 100:1, flowrate $=0.5 \mathrm{~mL} / \mathrm{min}, \lambda=295 \mathrm{~nm}): t_{\mathrm{R}}=10.38 \min (S), t_{\mathrm{R}}=13.04 \min (R) ;{ }^{1} \mathrm{H} \mathrm{NMR}\left(400 \mathrm{MHz}, \mathrm{CDCl}_{3}\right)$ : $\delta=7.73-7.77(\mathrm{~m}, 1 \mathrm{H}), 7.29-7.37(\mathrm{~m}, 4 \mathrm{H}), 7.08-7.25$ (m, $10 \mathrm{H}), 6.20$ (d, J=15.9 Hz, $1 \mathrm{H}), 6.13$ (ddd, $\mathrm{J}=15.8,6.5,6.5 \mathrm{~Hz}, 1 \mathrm{H}$ ), 3.07 (dd, J=14.1, $0.9 \mathrm{~Hz}, 1 \mathrm{H}), 3.02(\mathrm{~s}, 2 \mathrm{H}), 2.79$ (dd, J=13.9, $2.1 \mathrm{~Hz}, 1 \mathrm{H}$ ), $2.32\left(\mathrm{~m}_{\mathrm{c}}, 2 \mathrm{H}\right), 0.89$ (t, J=7.9 Hz, $\left.9 \mathrm{H}\right), 0.55$ (q, J=7.9 Hz, $\left.6 \mathrm{H}\right) \mathrm{ppm} ;{ }^{13} \mathrm{C} \mathrm{NMR}\left(100 \mathrm{MHz}, \mathrm{CDCl}_{3}\right)$ : $\delta=138.0,137.8,135.8,135.6,135.2,132.9,129.8,129.4,128.4,128.2,127.8,126.8,126.7,126.4$, 126.3, 126.0, 125.6, 123.9, 75.1, 43.8, 43.6, 40.5, 7.2, 6.9 ppm; IR $\left(\mathrm{CHCl}_{3}\right): \mathrm{v}=3688,3026,2959$, 2878, 1772, 1699, 1652, 1521, 1421, 1232, 1109, 1016, $929 \mathrm{~cm}^{-1}$; MS (EI): $\mathrm{m} / \mathrm{z}$ (\%): 349 (79) [(M-C $\left.\left.{ }_{9} \mathrm{H}_{9}\right)^{+}\right]$; HRMS (EI): $\mathrm{m} / z$ calcd for $\mathrm{C}_{23} \mathrm{H}_{29} \mathrm{OSi}\left[\left(\mathrm{M}-\mathrm{C}_{9} \mathrm{H}_{9}\right)^{+}\right]$: 349.19932; found: 349.19877.

\section{(R,E,E)-1-Benzylidene-3-methoxy-3-(3-phenyl-2-propenyl)-1,2,3,4-tetrahydronaphthalene}

[(R,E,E)-15]: According to the procedure described for $(R, E, E)-\mathbf{1 3}$, Heck reaction of $(E, E)-\mathbf{1 1}(0.230 \mathrm{~g}$, $0.445 \mathrm{mmol}$ ) with $\mathrm{Pd}(\mathrm{OAc})_{2}(5.0 \mathrm{mg}, 0.022 \mathrm{mmol}, 5.0 \mathrm{~mol} \%)$, (R)-BINAP $(0.021 \mathrm{~g}, 0.033 \mathrm{mmol}, 7.5$ $\mathrm{mol} \%$ ) and degassed 2,2,6,6-tetramethylpiperidine $(0.300 \mathrm{~mL}, 0.249 \mathrm{~g}, 1.76 \mathrm{mmol})$ in toluene $(4.5 \mathrm{~mL})$ at $50^{\circ} \mathrm{C}$ for $36 \mathrm{~h}$ afforded after purification by flash column chromatography on silica gel (cyclohexane/t-butyl methyl ether 98:2) desired $(R, E, E)-15(0.160 \mathrm{~g}, 98 \%)$ as a colorless oil. The enantiomeric excess was determined by HPLC analysis ( $97 \%$ ee). As determined from the ${ }^{1} \mathrm{H}$ NMR spectra, $(R, E, E)-15$ was isomerically pure. $R_{\mathrm{f}}=0.55$ (cyclohexane/t-butyl methyl ether 9:1); HPLC (Daicel Chiracel AD-H, $n$-heptane/i-PrOH 95:5, flow rate $=1 \mathrm{~mL} / \mathrm{min}, \lambda=295 \mathrm{~nm}$ ): $t_{\mathrm{R}}=9.21 \mathrm{~min}(S)$, $t_{\mathrm{R}}=10.17 \mathrm{~min}(R) ;[\alpha]_{\mathrm{D}}{ }^{20}=-60.9\left(c=0.960, \mathrm{CHCl}_{3}, 97 \%\right.$ ee $) ;{ }^{1} \mathrm{H}$ NMR $\left(400 \mathrm{MHz}, \mathrm{CDCl}_{3}\right): \delta=7.74-7.77$ (m, 1 H), 7.33-7.40 (m, 4 H), 7.17-7.29 (m, 9 H), 7.14-7.16 (m, 1 H), 6.34 (d, J=15.9 Hz, 1 H), 6.13 (ddd, J=15.8, 7.7, $6.8 \mathrm{~Hz}, 1 \mathrm{H}$ ), $3.18(\mathrm{~s}, 3 \mathrm{H}), 3.06(\mathrm{~d}, \mathrm{~J}=16.3 \mathrm{~Hz}, 1 \mathrm{H}), 3.00(\mathrm{~m}, 2 \mathrm{H}), 2.90$ (d, J=14.5 $\mathrm{Hz}, 1 \mathrm{H}$ ), 2.48 (ddd, $J=14.3,6.5,1.4 \mathrm{~Hz}, 1 \mathrm{H}$ ), 2.41 (ddd, $J=14.7,7.8,1.2 \mathrm{~Hz}, 1 \mathrm{H}$ ) ppm; ${ }^{13} \mathrm{C}$ NMR (100 $\left.\mathrm{MHz}, \mathrm{CDCl}_{3}\right): \delta=137.9,137.5,135.3,135.0,134.8,133.0,129.8,129.4,128.4,128.3,127.9,127.1$, 126.7, 126.4, 126.2, 125.8, 125.2, 124.0, 76.2, 49.2, 39.9, 38.5, 35.9 ppm; IR $\left(\mathrm{CHCl}_{3}\right)$ : v=3694, 3027 , 2930, 2831, 1950, 1600, 1495, 1455, 1197, 1088, $969 \mathrm{~cm}^{-1} ; \mathrm{MS}\left(\mathrm{Cl}-\mathrm{NH}_{3}\right): \mathrm{m} / \mathrm{z}(\%): 384$ (70) $\left[\left(\mathrm{M}+\mathrm{NH}_{4}\right)^{+}\right]$; elemental analysis calcd (\%) for $\mathrm{C}_{27} \mathrm{H}_{26} \mathrm{O}: \mathrm{C} 88.48, \mathrm{H} 7.15$; found: $\mathrm{C}$ 88.33, H 7.31. 
(S*,E,E)-1-Benzylidene-3-(3-phenyl-2-propenyl)-1,2,3,4-tetrahydronaphthalene $\left[\left(S^{*}, E, E\right)-16\right]:$ In analogy to the typical procedure described for $(R, E, E)-13$, Heck reaction of $(E, E)-12$ (36.5 mg, 0.0750 mmol) with $\mathrm{Pd}(\mathrm{OAc})_{2}$ (0.84 mg, $0.0037 \mathrm{mmol}, 5.0 \mathrm{~mol} \%$ ), (R)-BINAP (3.5 mg, $0.0056 \mathrm{mmol}, 7.5 \mathrm{~mol} \%$ ) and $\mathrm{K}_{2} \mathrm{CO}_{3}(0.042 \mathrm{~g}, 0.30 \mathrm{mmol})$ in toluene $(0.75 \mathrm{~mL})$ at $80^{\circ} \mathrm{C}$ for $15 \mathrm{~h}$ provided after purification by flash column chromatography on silica gel (cyclohexane/t-butyl methyl ether 200:1) $\left(S^{*}, E, E\right)-16(17.9$ $\mathrm{mg}, 71 \%)$ as a colorless oil. The enantiomeric excess was determined by HPLC analysis (18\% ee). As determined from the ${ }^{1} \mathrm{H}$ NMR spectra, $\left(S^{*}, E, E\right)-16$ was isomerically pure. $R_{\mathrm{f}}=0.25$ (cyclohexane); HPLC (Daicel Chiracel AD-H, $n$-heptane/i-PrOH 200:1, flow-rate $=0.8 \mathrm{~mL} / \mathrm{min}, \lambda=295 \mathrm{~nm}$ ): $t_{\mathrm{R}}=11.27$ (minor), $t_{\mathrm{R}}=15.00$ (major); ${ }^{1} \mathrm{H}$ NMR (500 MHz, $\mathrm{CDCl}_{3}$ ): $\delta=7.73(\mathrm{~m}, 1 \mathrm{H}), 7.33-7.39(\mathrm{~m}, 4 \mathrm{H}), 7.18-7.30$ (m, 8 H), 7.10-7.14 (m, 2 H), 6.37 (d, J=15.7 Hz, 1 H), 6.17 (ddd, J=15.5, 7.6, 7.6 Hz, 1 H), 3.08 (dd, J=14.5, 3.8 Hz, $1 \mathrm{H}$ ), 2.98 (dd, J=16.2, $4.5 \mathrm{~Hz}, 1 \mathrm{H}$ ), 2.63 (dd, J=16.3, $9.7 \mathrm{~Hz}, 1 \mathrm{H}$ ), 2.44 (ddd, J=14.5, 10.3, $2.0 \mathrm{~Hz}, 1 \mathrm{H}$ ), 2.31 (dddd, J=13.9, 6.8, 6.8, $1.0 \mathrm{~Hz}, 1 \mathrm{H}$ ), 2.23 (ddd, J=14.1, 7.2, 7.2 Hz, 1 H), 2.02 $\left(\mathrm{m}_{\mathrm{c}}, 1 \mathrm{H}\right) \mathrm{ppm} ;{ }^{13} \mathrm{C}$ NMR $\left(125 \mathrm{MHz}, \mathrm{CDCl}_{3}\right): \delta=138.2,137.7,137.1,136.7,136.2,131.7,129.5,129.4$, $128.5,128.3,127.6,127.0,126.5,126.5,126.3,126.1,124.4,124.2,39.3,36.3,35.2,33.9$ ppm; IR $\left(\mathrm{CHCl}_{3}\right): \mathrm{v}=3678,3015,2921,1807,1700,1521,1422,1216,967,928,801 \mathrm{~cm}^{-1} ; \mathrm{MS}(\mathrm{EI}): \mathrm{m} / \mathrm{z}(\%):$ 336 (59) $\left[\mathrm{M}^{+}\right]$; elemental analysis calcd (\%) for $\mathrm{C}_{27} \mathrm{H}_{26} \mathrm{O}: \mathrm{C} 92.81, \mathrm{H}$ 7.19; found: $\mathrm{C}$ 92.51, H 6.95.

$\left(R^{*}, E, E\right)-3-B e n z y l i d e n e-1-(3-p h e n y l-2-p r o p e n y l)-1-i n d a n o l \quad\left[\left(R^{*}, E, E\right)-17\right]: \quad$ According to the procedure described for $(R, E, E)-13$, Heck reaction of $(E, E)-8(0.101 \mathrm{~g}, 0.207 \mathrm{mmol})$ with $\mathrm{Pd}(\mathrm{OAc})_{2}$ (2.3 mg, $0.010 \mathrm{mmol}, 5.0 \mathrm{~mol} \%$ ), (R)-BINAP (9.6 mg, $0.015 \mathrm{mmol}, 7.5 \mathrm{~mol} \%$ ) and 2,2,6,6tetramethylpiperidine $(0.141 \mathrm{~mL}, 0.117 \mathrm{~g}, 0.825 \mathrm{mmol})$ in toluene $(2.7 \mathrm{~mL})$ at $80^{\circ} \mathrm{C}$ for $15 \mathrm{~h}$ gave after purification by flash column chromatography on silica gel (cyclohexane/t-butyl methyl ether 9:1) $\left(R^{*}, E, E\right)-17(0.061 \mathrm{~g}, 87 \%)$ as a colorless oil. The enantiomeric excess was determined by HPLC analysis $\left(26 \%\right.$ ee). As determined from the ${ }^{1} \mathrm{H}$ NMR spectra, $\left(R^{\star}, E, E\right)-17$ was isomerically pure. $R_{\mathrm{f}}=0.30$ (cyclohexane/t-butyl methyl ether 3:1); HPLC (Daicel Chiracel AD, $n$-heptane/i-PrOH 9:1, flowrate $=1 \mathrm{~mL} / \mathrm{min}, \lambda=295 \mathrm{~nm}$ ): $t_{\mathrm{R}}=15.59$ (major), $t_{\mathrm{R}}=22.61$ (minor); $[\alpha]_{\mathrm{D}}{ }^{20}=-12.6$ ( $c=0.198, \mathrm{CHCl}_{3}, 26 \%$ ee); ${ }^{1} \mathrm{H}$ NMR $\left(500 \mathrm{MHz}, \mathrm{CDCl}_{3}\right): \delta=7.63(\mathrm{dd}, \mathrm{J}=6.9,0.4 \mathrm{~Hz}, 1 \mathrm{H}), 7.44-7.48(\mathrm{~m}, 3 \mathrm{H}), 7.35-7.41(\mathrm{~m}, 4$ H), 7.31-7.35 (m, 3 H), 7.27-7.31 (m, $2 \mathrm{H}), 7.23(\mathrm{tt}, \mathrm{J}=7.1,1.8 \mathrm{~Hz}, 1 \mathrm{H}), 7.04$ (dd, J=2.4, 2.4 Hz, $1 \mathrm{H}$ ), $6.52(\mathrm{~d}, \mathrm{~J}=15.8 \mathrm{~Hz}, 1 \mathrm{H}$ ), 6.29 (ddd, J=15.8, 7.4, 7.4 Hz, $1 \mathrm{H}$ ), 3.42 (dd, J=16.9, $2.1 \mathrm{~Hz}, 1 \mathrm{H}$ ), 3.18 (dd, J=16.9, $2.5 \mathrm{~Hz}, 1 \mathrm{H}$ ), 2.83 (ddd, J=13.9, 7.6, 1.0 Hz, $1 \mathrm{H}$ ), 2.73 (ddd, J=13.9, 7.1, 1.3 Hz, $1 \mathrm{H}$ ), 2.29 (s, $1 \mathrm{H}) \mathrm{ppm} ;{ }^{13} \mathrm{C}$ NMR $\left(125 \mathrm{MHz}, \mathrm{CDCl}_{3}\right): \delta=148.1,141.5,139.0,137.7,137.3,134.2,129.0,128.8$, 128.7, 128.6, 128.6, 127.4, 126.8, 126.3, 124.9, 123.6, 120.8, 120.3, 81.6, 46.5, 45.2 ppm; IR $\left(\mathrm{CHCl}_{3}\right)$ : $v=3680,3590,3021,1947,1802,1647,1597,1493,1423,1223,1023,924,799 \mathrm{~cm}^{-1} ; \mathrm{MS}\left(\mathrm{Cl}-\mathrm{NH}_{3}\right)$ : $\mathrm{m} / \mathrm{z}(\%): 354(2)\left[\left(\mathrm{M}+\mathrm{NH}_{4}\right)^{+}\right], 321(100)\left[(\mathrm{M}-\mathrm{OH})^{+}\right]$; HRMS (EI): $\mathrm{m} / \mathrm{z}$ calcd for $\mathrm{C}_{25} \mathrm{H}_{20}\left[\left(\mathrm{M}-\mathrm{H}_{2} \mathrm{O}\right)^{+}\right]$: 320.15652; found: 320.15650 .

\section{(R,Z,Z)-4-Benzylidene-2-(3-phenyl-2-propenyl)-1,2,3,4-tetrahydro-2-naphthalenol}

$[(R, Z, Z)-13]:$ According to the procedure described for $(R, E, E)-13$, Heck reaction of $(Z, Z)-9(0.090 \mathrm{~g}, 0.18 \mathrm{mmol})$ with $\mathrm{Pd}(\mathrm{OAc})_{2}(2.0 \mathrm{mg}, 0.0089 \mathrm{mmol}, 5.0 \mathrm{~mol} \%),(R)$-BINAP $(8.4 \mathrm{mg}, 0.014 \mathrm{mmol}, 7.5 \mathrm{~mol} \%)$ and 2,2,6,6-tertamethylpiperidine $(0.12 \mathrm{~mL}, 0.10 \mathrm{~g}, 0.72 \mathrm{mmol})$ in toluene $(1.8 \mathrm{~mL})$ at $100^{\circ} \mathrm{C}$ for $20 \mathrm{~h}$ afforded after purification by flash column chromatography on silica gel (cyclohexane/t-butyl methyl 
ether 9:1) $(R, Z, Z)-13(0.039 \mathrm{~g}, 61 \%)$ along with the 2(2E)-isomer $(R, Z, E)-13(3.7 \mathrm{mg}, 6 \%)$ and the $4 E-$ isomer $(R, E, Z)-13(1.8 \mathrm{mg}, 3 \%)$ as a colorless oil. The enantiomeric excess was determined by HPLC analysis $\left(33 \%\right.$ ee). $R_{\mathrm{f}}=0.31$ (cyclohexane/t-butyl methyl ether 3:1); HPLC (Daicel Chiracel AD-H, $n$ heptane $/ i-\mathrm{PrOH}$ 9:1, flow-rate=1 mL/min, $\lambda=295 \mathrm{~nm}): t_{\mathrm{R}}=8.55(S), t_{\mathrm{R}}=11.63(R) ;[\alpha]_{\mathrm{D}}{ }^{20}=-24.9(c=1.19$, $\mathrm{CHCl}_{3}, 19 \%$ ee); ${ }^{1} \mathrm{H}$ NMR $\left(400 \mathrm{MHz}, \mathrm{CDCl}_{3}\right): \delta=7.20\left(\mathrm{~m}_{\mathrm{c}}, 4 \mathrm{H}\right), 7.06-7.15(\mathrm{~m}, 7 \mathrm{H}), 6.99-7.03(\mathrm{~m}, 2$ H), 6.72-6.77 (m, $1 \mathrm{H}$ ), 6.58 (ddd, J=11.8, 1.8, $1.8 \mathrm{~Hz}, 1 \mathrm{H}$ ), 6.42 (s, $1 \mathrm{H}$ ), 5.87 (ddd, J=11.8, 7.3, 7.3 $\mathrm{Hz}, 1 \mathrm{H}$ ), 2.91 (s, $2 \mathrm{H}$ ), 2.63 (ddd, J=15.0, 7.4, $1.8 \mathrm{~Hz}, 1 \mathrm{H}$ ), 2.61 (ddd, J=15.0, 7.3, 1.8 Hz, $1 \mathrm{H}$ ), 2.52 (dd, J=12.9, $1.6 \mathrm{~Hz}, 1 \mathrm{H}), 2.46(\mathrm{~d}, \mathrm{~J}=13.0 \mathrm{~Hz}, 1 \mathrm{H}), 1.91$ (br s, $1 \mathrm{H}) \mathrm{ppm} ;{ }^{13} \mathrm{C}$ NMR $\left(100 \mathrm{MHz}, \mathrm{CDCl}_{3}\right)$ : $\delta=137.9,137.4,136.1,134.2,133.5,132.2,129.5,129.0,128.8,128.7,128.4,128.3,128.3,128.1$, 126.8, 126.7, 125.0, 72.2, 47.4, 42.9, 40.1 ppm; IR $\left(\mathrm{CDCl}_{3}\right)$ : v=3575, 3062, 3025, 2910, 1952, 1763, $1600,1494,1446,1367,1251,1074 \mathrm{~cm}^{-1}$; MS (EI): $\mathrm{m} / \mathrm{z}(\%): 352(27)\left[\mathrm{M}^{+}\right]$; elemental analysis calcd (\%) for $\mathrm{C}_{26} \mathrm{H}_{24} \mathrm{O}$ : C 88.60, H 6.86; found: C 88.33, H 7.01.

\section{(R,Z,Z)-1-Benzylidene-3-methoxy-3-(3-phenyl-2-propenyl)-1,2,3,4-tetrahydronaphthalene}

[(R,Z,Z)-15]: Following the procedure described for $(R, E, E)-13$, Heck reaction of $(Z, Z)-11(0.053 \mathrm{~g}$, $0.10 \mathrm{mmol}$ ) with $\mathrm{Pd}(\mathrm{OAc})_{2}(1.1 \mathrm{mg}, 0.0050 \mathrm{mmol}, 5.0 \mathrm{~mol} \%),(R)$-BINAP $(4.7 \mathrm{mg}, 0.0075 \mathrm{mmol}, 7.5$ $\mathrm{mol} \%)$ and 2,2,6,6-tetramethylpiperidine $(0.070 \mathrm{~mL}, 0.058 \mathrm{~g}, 0.41 \mathrm{mmol})$ in toluene $(1.0 \mathrm{~mL})$ at $80^{\circ} \mathrm{C}$ for $20 \mathrm{~h}$ afforded after purification by flash column chromatography on silica gel (cyclohexane/t-butyl methyl ether 99:1) desired $(R, Z, Z)-15(0.029 \mathrm{~g}, 80 \%)$ as a colorless oil. The enantiomeric excess was determined by HPLC analysis (38\% ee). As determined from the ${ }^{1} \mathrm{H}$ NMR spectra, $(R, Z, Z)-15$ was isomerically pure. $R_{\mathrm{f}}=0.56$ (cyclohexane/t-butyl methyl ether 9:1); HPLC (Daicel Chiracel AD-H, $n$ heptane/i-PrOH 97:3, flow-rate $=0.8 \mathrm{~mL} / \mathrm{min}, \lambda=295 \mathrm{~nm}): t_{\mathrm{R}}=6.17(S), t_{\mathrm{R}}=7.00(R) ;[\alpha]_{436}{ }^{20}=-7.24$ $\left(c=1.05, \mathrm{CHCl}_{3}, 38 \%\right.$ ee $) ;{ }^{1} \mathrm{H}$ NMR $\left(400 \mathrm{MHz}, \mathrm{CDCl}_{3}\right): \delta=7.23-7.27(\mathrm{~m}, 4 \mathrm{H}), 7.08-7.22(\mathrm{~m}, 9 \mathrm{H})$, 6.79-6.84 (m, $1 \mathrm{H}), 6.63$ (ddd, J=11.8, 1.9, $1.9 \mathrm{~Hz}, 1 \mathrm{H}$ ), 6.46 (s, $1 \mathrm{H}$ ), 5.92 (ddd, J=11.8, 6.9, $6.9 \mathrm{~Hz}$, $1 \mathrm{H}$ ), 3.27 (s, $3 \mathrm{H}$ ), 3.07 (d, J=16.8 Hz, 1 H), 2.95 (d, J=16.7 Hz, 1 H), 2.67-2.79 (m, 3 H), 2.59 (ddd, $\mathrm{J}=12.9,1.0,1.0 \mathrm{~Hz}, 1 \mathrm{H}) \mathrm{ppm} ;{ }^{13} \mathrm{C}$ NMR $\left(100 \mathrm{MHz}, \mathrm{CDCl}_{3}\right): \delta=138.5,137.6,136.4,134.8,133.9$, $131.5,129.3,128.9,128.7,128.7,128.3,128.2,127.7,127.1,126.7,126.5,124.9,76.5,49.1$, 43.9, 40.2, 34.4 ppm; IR $\left(\mathrm{CDCl}_{3}\right): \mathrm{v}=3061,3024,2939,2830,1951,1764,1599,1494,1446,1252,1087$ $\mathrm{cm}^{-1}$; MS $\left(\mathrm{Cl}-\mathrm{NH}_{3}\right): \mathrm{m} / \mathrm{z}(\%): 384(13)\left[\left(\mathrm{M}+\mathrm{NH}_{4}\right)^{+}\right]$; elemental analysis calcd (\%) for $\mathrm{C}_{27} \mathrm{H}_{26} \mathrm{O}: \mathrm{C} 88.48$, H 7.15; found: C 88.19, H 7.24.

(1S,3R)-3-(2-Hydroxyethyl)-3-methoxy-1,2,3,4-tetrahydro-1-naphthalenol $\quad[(1 S, 3 R)-S \mid 21]: \quad$ At $-78^{\circ} \mathrm{C}$, a steady stream of ozone and oxygen was bubbled through a solution of $(R, E, E)-15(0.140 \mathrm{~g}$, $0.382 \mathrm{mmol})$ in dry $\mathrm{CH}_{2} \mathrm{Cl}_{2}(4 \mathrm{~mL})$ and dry $\mathrm{MeOH}(4 \mathrm{~mL})$ until the solution turned bluish. Pure oxygen ( $5 \mathrm{~min}$ ) and then nitrogen ( $20 \mathrm{~min}$ ) were bubbled through the solution to remove any traces of ozone. An excess of $\mathrm{NaBH}_{4}(0.145 \mathrm{~g}, 3.82 \mathrm{mmol})$ was added in one portion and the mixture was allowed to warm slowly to room temperature overnight; it was then diluted with $\mathrm{CH}_{2} \mathrm{Cl}_{2}(20 \mathrm{~mL})$ and carefully quenched with aq $\mathrm{HCl}(2 \mathrm{M}, 8 \mathrm{~mL})$. The aqueous layer was extracted with $\mathrm{CH}_{2} \mathrm{Cl}_{2}(20 \mathrm{~mL})$ and the combined organic phases were washed with saturated aq $\mathrm{NaHCO}_{3}(5 \mathrm{~mL})$, dried $\left(\mathrm{Na}_{2} \mathrm{SO}_{4}\right)$ and evaporated under reduced pressure. Purification of the residue by flash column chromatography on silica gel ( $t$-butyl methyl ether/MeOH 99.5:0.5) allowed partial removal of the minor diastereomer and 
afforded (1S,3R)-SI21 (0.049 g, 58\%, dr>99:1) along with (1S,3R)-SI21 (0.022 g, 26\%, dr=72:28) as colorless oils. The overall diasteromeric ratio was determined by the ${ }^{1} \mathrm{H}$ NMR spectra of crude (1S,3R)-SI21 (dr=91:9). $R_{\mathrm{f}}=0.20$ ( $t$-butyl methyl ether); ${ }^{1} \mathrm{H}$ NMR $\left(400 \mathrm{MHz}, \mathrm{CDCl}_{3}\right): \delta=7.49$ (dd, $J=7.3$, $1.8 \mathrm{~Hz}, 1 \mathrm{H}$ ), 7.27 (dddd, J=6.8, 6.8, 1.3, $0.5 \mathrm{~Hz}, 1 \mathrm{H}$ ), 7.23 (ddd, J=7.3, 7.3, 1.7 Hz, $1 \mathrm{H}$ ), 7.10 (d, $\mathrm{J}=7.4 \mathrm{~Hz}, 1 \mathrm{H}$ ), 4.73 (br s, $1 \mathrm{H}$ ), 3.92 (ddd, J=11.1, 7.4, $6.3 \mathrm{~Hz}, 1 \mathrm{H}$ ), 3.85 (ddd, J=11.0, 6.6, $5.9 \mathrm{~Hz}, 1$ H), 3.65 (br s, 1 H), 3,16 (s, 3 H), 3.15 (dd, J=17.2, $2.5 \mathrm{~Hz}, 1 \mathrm{H}$ ), 2.68 (d, J=17.2 Hz, 1 H), 2.47 (ddd, J=14.3, 2.7, $2.7 \mathrm{~Hz}, 1 \mathrm{H}$ ), 2.19 (br s, $1 \mathrm{H}$ ), 2.14 (ddd, J=14.8, 7.2, 7.2 Hz, 1 H), 1.98 (dd, J=14.3, 5.2 $\mathrm{Hz}, 1 \mathrm{H}$ ), 1.68 (ddd, $\mathrm{J}=14.8,6.0,6.0 \mathrm{~Hz}, 1 \mathrm{H}$ ) ppm; ${ }^{13} \mathrm{C}$ NMR $\left(100 \mathrm{MHz}, \mathrm{CDCl}_{3}\right): \delta=138.6,132.1$, 129.9, 128.8, 128.0, 127.0, 76.9, 67.7, 58.5, 49.3, 39.5, 37.8, 37.3 ppm; IR $\left(\mathrm{CDCl}_{3}\right): \mathrm{v}=3623,3510$, 2943, 1749, 1457, 1254, 1080, $911 \mathrm{~cm}^{-1}$; MS $\left(\mathrm{Cl}_{-} \mathrm{NH}_{3}\right): \mathrm{m} / \mathrm{z}(\%): 222(73)\left[\mathrm{M}^{+}\right]$; elemental analysis calcd (\%) for $\mathrm{C}_{13} \mathrm{H}_{18} \mathrm{O}_{3}$ : C 70.24, $\mathrm{H}$ 8.16; found: C 69.98, $\mathrm{H} 8.46$.

$(1 R, 3 R)$-SI21: $R_{\mathrm{f}}=0.17$ ( $t$-butyl methyl ether); ${ }^{1} \mathrm{H}$ NMR $\left(400 \mathrm{MHz}, \mathrm{CDCl}_{3}\right): \delta=7.53$ (ddd, $\mathrm{J}=7.7,1.5,0.6$ $\mathrm{Hz}, 1 \mathrm{H}), 7.23\left(\mathrm{~m}_{\mathrm{c}}, 2 \mathrm{H}\right), 7.10$ (dd, J=7.1, $\left.0.9 \mathrm{~Hz}, 1 \mathrm{H}\right), 4.97$ (dd, J=8.6, $\left.5.9 \mathrm{~Hz}, 1 \mathrm{H}\right), 3.88\left(\mathrm{~m}_{\mathrm{c}}, 2 \mathrm{H}\right)$, 3.23 (s, $3 \mathrm{H}$ ), 3.01 (dd, J=16.7, $1.7 \mathrm{~Hz}, 1 \mathrm{H}$ ), 2.86 (d, J=16.7 Hz, $1 \mathrm{H}$ ), 2.50 (ddd, J=13.6, 6.0, $2.0 \mathrm{~Hz}, 1$ H), 2.09 (br s, 2 H), 1.95 (ddd, J=14.8, 6.6, $6.1 \mathrm{~Hz}, 1 \mathrm{H}$ ), 1.89 (ddd, J=14.9, 6.2, 6.2 Hz, 1 H), 1.80 (dd, $\mathrm{J}=13.6,8.7 \mathrm{~Hz}, 1 \mathrm{H}) \mathrm{ppm} ;{ }^{13} \mathrm{C} \mathrm{NMR}\left(100 \mathrm{MHz}, \mathrm{CDCl}_{3}\right): \delta=138.7,133.7,129.0,127.8,126.9,126.7$, $76.4,67.3,58.8,49.4,40.7,38.9,37.7$ ppm.

(1S,3R)-4-bromobenzoic acid 3-[2-(4-bromobenzoyloxy)ethyl]-3-methoxy-1,2,3,4-tetrahydro-1naphthalenyl ester [(1S,3R)-SI22]: At ambient temperature, a solution of diol $(1 S, 3 R)-S \mid 21(47.0 \mathrm{mg}$, $0.211 \mathrm{mmol})$ and dry $\mathrm{CH}_{2} \mathrm{Cl}_{2}(5 \mathrm{~mL})$ and $\mathrm{Et}_{3} \mathrm{~N}(0.12 \mathrm{~mL}, 0.085 \mathrm{~g}, 0.85 \mathrm{mmol})$ were added successively to a solution 4-bromobenzoyl chloride $(0.186 \mathrm{~g}, 0.846 \mathrm{mmol})$ and DMAP $(9.5 \mathrm{mg}, 0.084 \mathrm{mmol}, 40$ mol\%) and dry $\mathrm{CH}_{2} \mathrm{Cl}_{2}(3 \mathrm{~mL})$. The reaction mixture was maintained at this temperature overnight, then diluted with $t$-butyl methyl ether $(40 \mathrm{~mL})$, washed with aq $\mathrm{HCl}(2 \mathrm{M}, 20 \mathrm{~mL})$ and saturated aq $\mathrm{NaHCO}_{3}(20 \mathrm{~mL})$ and dried $\left(\mathrm{MgSO}_{4}\right)$. Evaporation of the solvents in vacuo and purification of the residue by flash column chromatography on silica gel (cyclohexane/t-butyl methyl ether 4:1) afforded (1S,3R)-SI22 (0.123 g, 99\%) as a colorless solid. $R_{\mathrm{f}}=0.51$ (cyclohexane/t-butyl methyl ether 3:1); m.p. $86^{\circ} \mathrm{C} ;[\alpha]_{D}{ }^{20}=-29.7\left(c=1.00, \mathrm{CHCl}_{3}, 97 \%\right.$ ee); ${ }^{1} \mathrm{H} \mathrm{NMR}\left(400 \mathrm{MHz}, \mathrm{CDCl}_{3}\right): \delta=7.94$ (ddd, J=8.7, 2.1, 2.1 $\mathrm{Hz}, 2 \mathrm{H}$ ), 7.88 (ddd, J=8.7, 2.1, 2.1 Hz, $2 \mathrm{H}$ ), $7.57\left(\mathrm{~m}_{\mathrm{c}}, 4 \mathrm{H}\right), 7.32$ (dd, J=7.4, $\left.1.7 \mathrm{~Hz}, 1 \mathrm{H}\right), 7.25\left(\mathrm{~m}_{\mathrm{c}}, 2\right.$ H), 7.16 (dd, J=7.4, $1.2 \mathrm{~Hz}, 1 \mathrm{H}$ ), 6.31 (dd, J=6.5, $6.5 \mathrm{~Hz}, 1 \mathrm{H}$ ), 4.53 (dd, J=7.2, 7.2 Hz, $2 \mathrm{H}$ ), 3.27 (s, 3 H), 3.17 (d, J=16.2 Hz, 1 H), 2.92 (d, J=16.2 Hz, 1 H), 2.40 (ddd, J=13.8, 6.3, $1.3 \mathrm{~Hz}, 1 \mathrm{H}$ ), 2.30 (dd, J=13.8, $6.8 \mathrm{~Hz}, 1 \mathrm{H}$ ), 2.15 (ddd, J=14.8, 7.2, 7.2 Hz, $1 \mathrm{H}$ ), 2.05 (ddd, J=14.8, 7.3, 7.3 Hz, $1 \mathrm{H}$ ) ppm; ${ }^{13} \mathrm{C}$ NMR $\left(100 \mathrm{MHz}, \mathrm{CDCl}_{3}\right): \delta=134.7,133.4,131.9,131.8,131.4,131.2,129.4,129.2,129.2,128.6$, 128.4, 128.2, 128.1, 126.9, 74.0, 70.5, 60.9, 49.4, 39.4, 36.9, 33.6 ppm; IR $\left(\mathrm{CDCl}_{3}\right): \mathrm{v}=3691,2943$, 1926, 1713, 1592, 1484, 1399, 1271, 1115, 1103, 1013, $848 \mathrm{~cm}^{-1}$; MS $\left(\mathrm{Cl}-\mathrm{NH}_{3}\right): \mathrm{m} / \mathrm{z}(\%): 608(22)$ $\left[\left(\mathrm{M}+\mathrm{NH}_{4}\right)^{+}\right], 606(41)\left[\left(\mathrm{M}+\mathrm{NH}_{4}\right)^{+}\right], 604(21)\left[\left(\mathrm{M}+\mathrm{NH}_{4}\right)^{+}\right]$; elemental analysis calcd (\%) for $\mathrm{C}_{27} \mathrm{H}_{24} \mathrm{Br}_{2} \mathrm{O}_{5}: \mathrm{C}$ 55.12, H 4.11; found: C 55.06, H 3.96 . 


\section{Determination of Absolute Configuration}

$(R, E, E)-13$ was correlated with $(R, E, E)-15$ by methylation using Meerwein's salt (Scheme 8$)$. Then, two-fold ozonolysis of $(R, E, E)-15\left(97 \%\right.$ ee) under standard conditions at $-78^{\circ} \mathrm{C}$ and subsequent treatment with excess $\mathrm{NaBH}_{4}$ (10 equiv) produced $(1 S, 3 R)$-SI21 (dr = 91:9) in high yield. ${ }^{11}$ After removal of the minor diastereomer (1R,3R)-SI21 (not shown) by flash column chromatography, derivatization of $(1 S, 3 R)$-SI21 with $p$-bromobenzoyl chloride afforded $(1 S, 3 R)$-SI22, which was crystallized from $i-\mathrm{Pr}_{2} \mathrm{O}$ at room temperature. The absolute and relative configuration was unambiguously secured by $\mathrm{X}$-ray analysis (see Section 6 ).

Scheme S14. Determination of the Absolute Configuration ${ }^{a}$

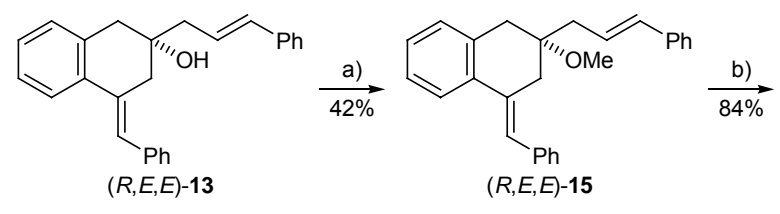<smiles>COC1(CCO)Cc2ccccc2[C@H](O)C1</smiles>

${ }^{a}$ Reaction conditions: a) $\mathrm{Me}_{3} \mathrm{O}^{+} \mathrm{BF}_{4}^{-}$, Proton-sponge ${ }^{\mathrm{TM}}, \mathrm{CH}_{2} \mathrm{Cl}_{2}, \mathrm{RT}$; b) i) $\mathrm{O}_{3}, \mathrm{CH}_{2} \mathrm{Cl}_{2}, \mathrm{MeOH},-78^{\circ} \mathrm{C}$; ii) $\mathrm{NaBH}_{4}$, $-78^{\circ} \mathrm{C} \rightarrow \mathrm{RT}$; c) 4-bromobenzoyl chloride, DMAP, $\mathrm{Et}_{3} \mathrm{~N}, \mathrm{CH}_{2} \mathrm{Cl}_{2}, \mathrm{RT}$.

\section{Molecular Structure of (1S,3R)-SI22}

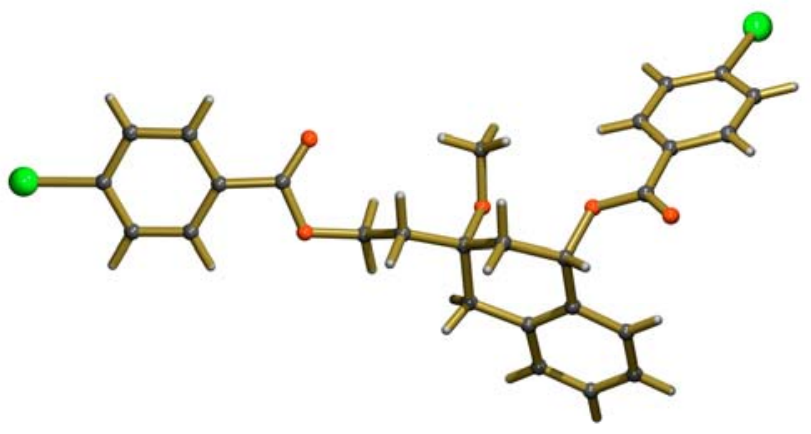

\footnotetext{
${ }^{11}$ (a) Broadhurst, M. J.; Hassal, C. H.; Thomas, G. J. J. Chem. Soc., Perkin Trans. 1 1982, 2239-2248. (b) Coogan, M. P.; Haigh, R.; Hall, A.; Harris, L. D.; Hibbs, D. E.; Jenkins, R. L.; Jones, C. L.; Tomkinson, N. C. O. Tetrahedron 2003, 59, 7389-7395.
} 


\section{Copies of NMR Spectra of Cyclization Precursors and Products}

$(E, E)-9\left({ }^{1} \mathrm{H}\right):$

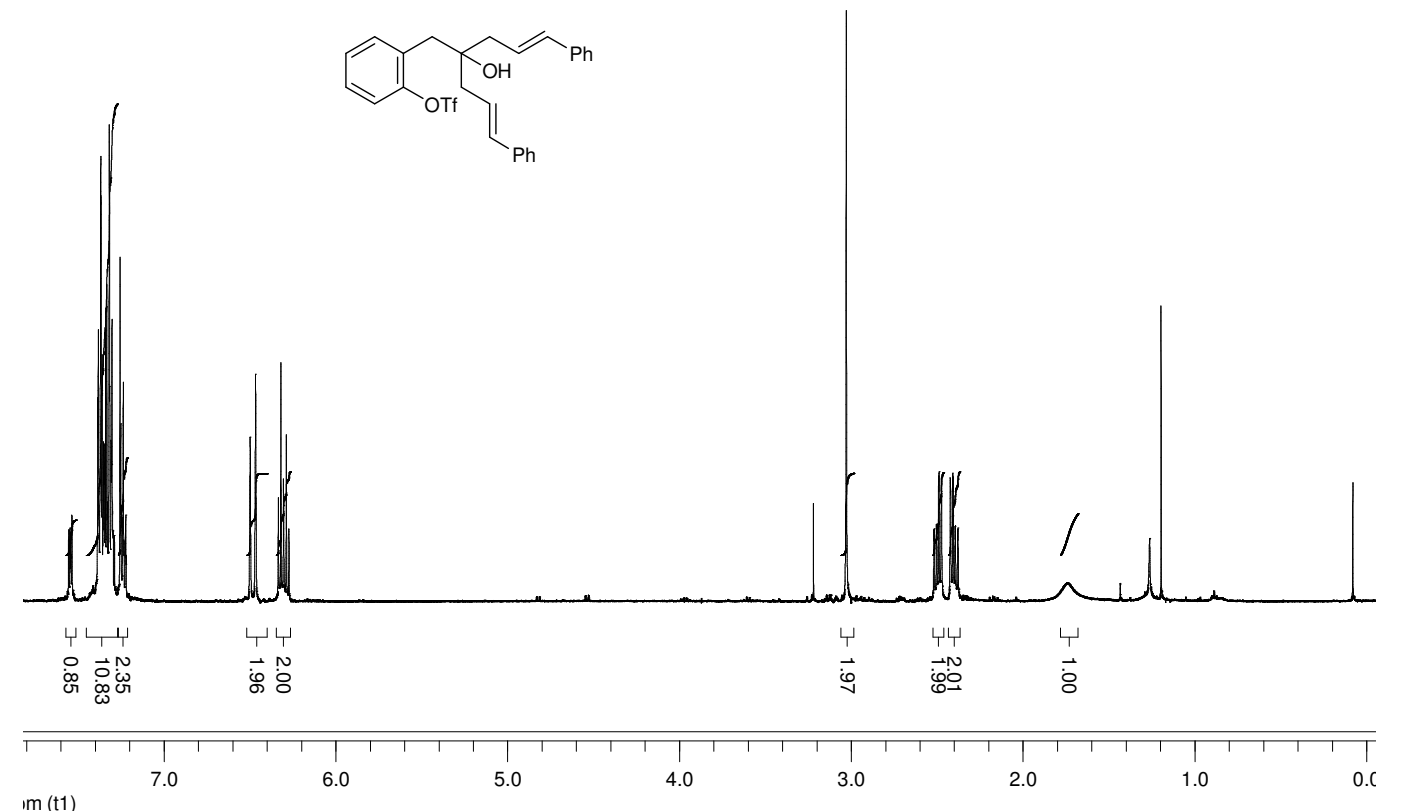

Im (t1)

$(E, E)-\mathbf{9}\left({ }^{13} \mathrm{C}\right)$ :
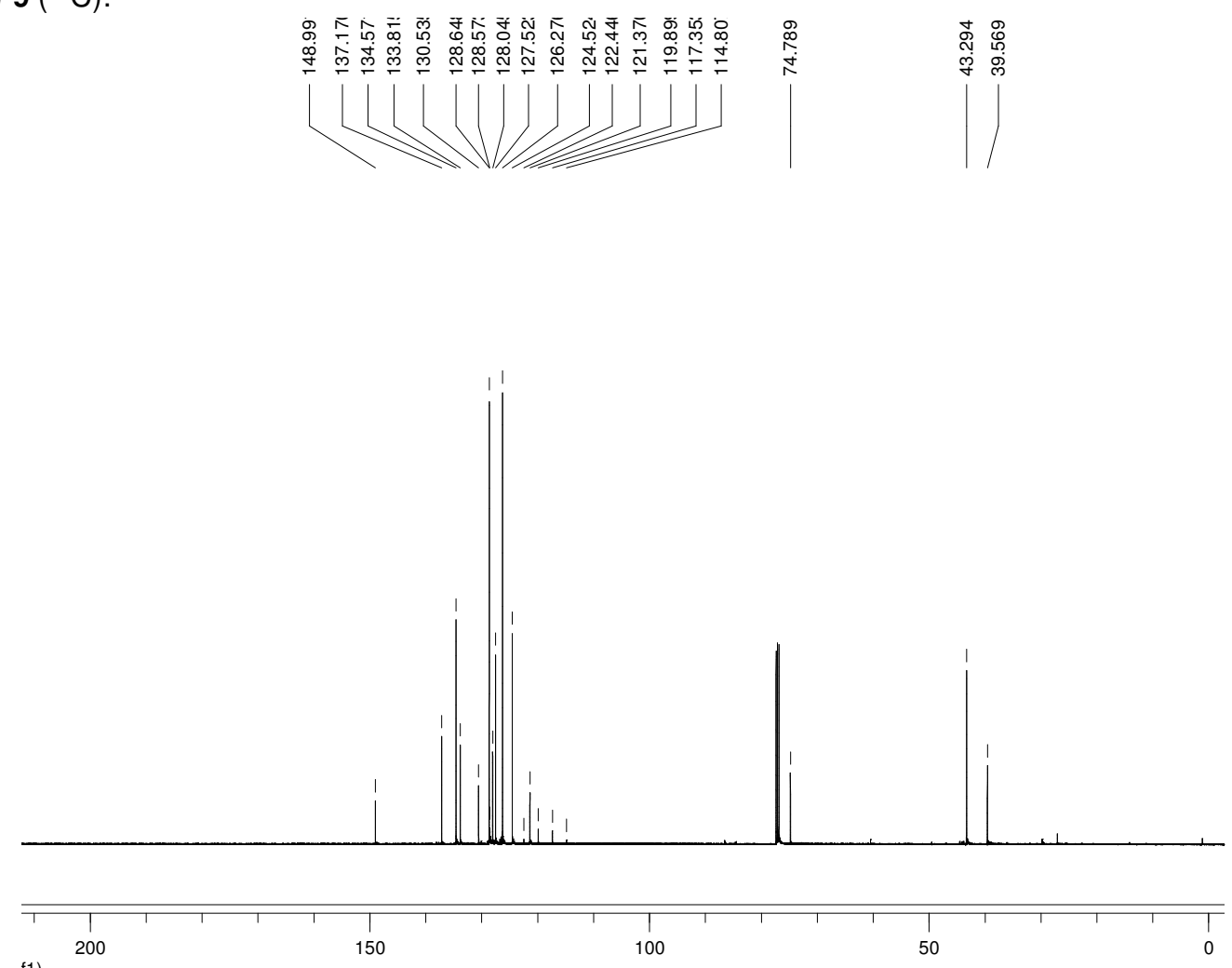

f1) 
$(R, E, E)-13\left({ }^{1} \mathrm{H}\right):$
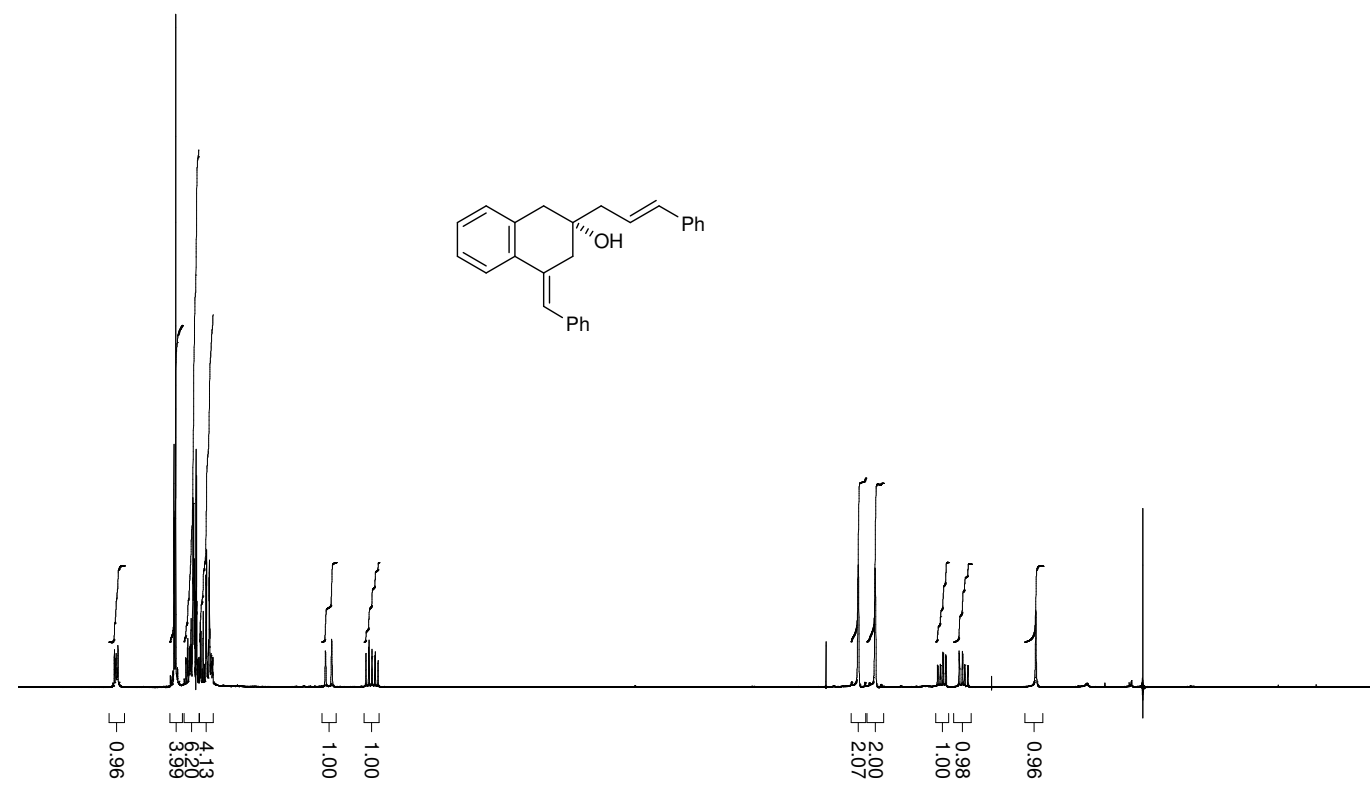

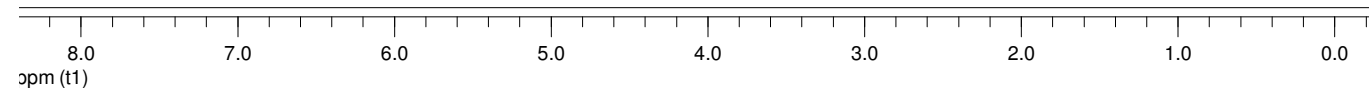

$(R, E, E)-13\left({ }^{13} \mathrm{C}\right):$

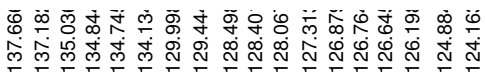
LLLLLLU
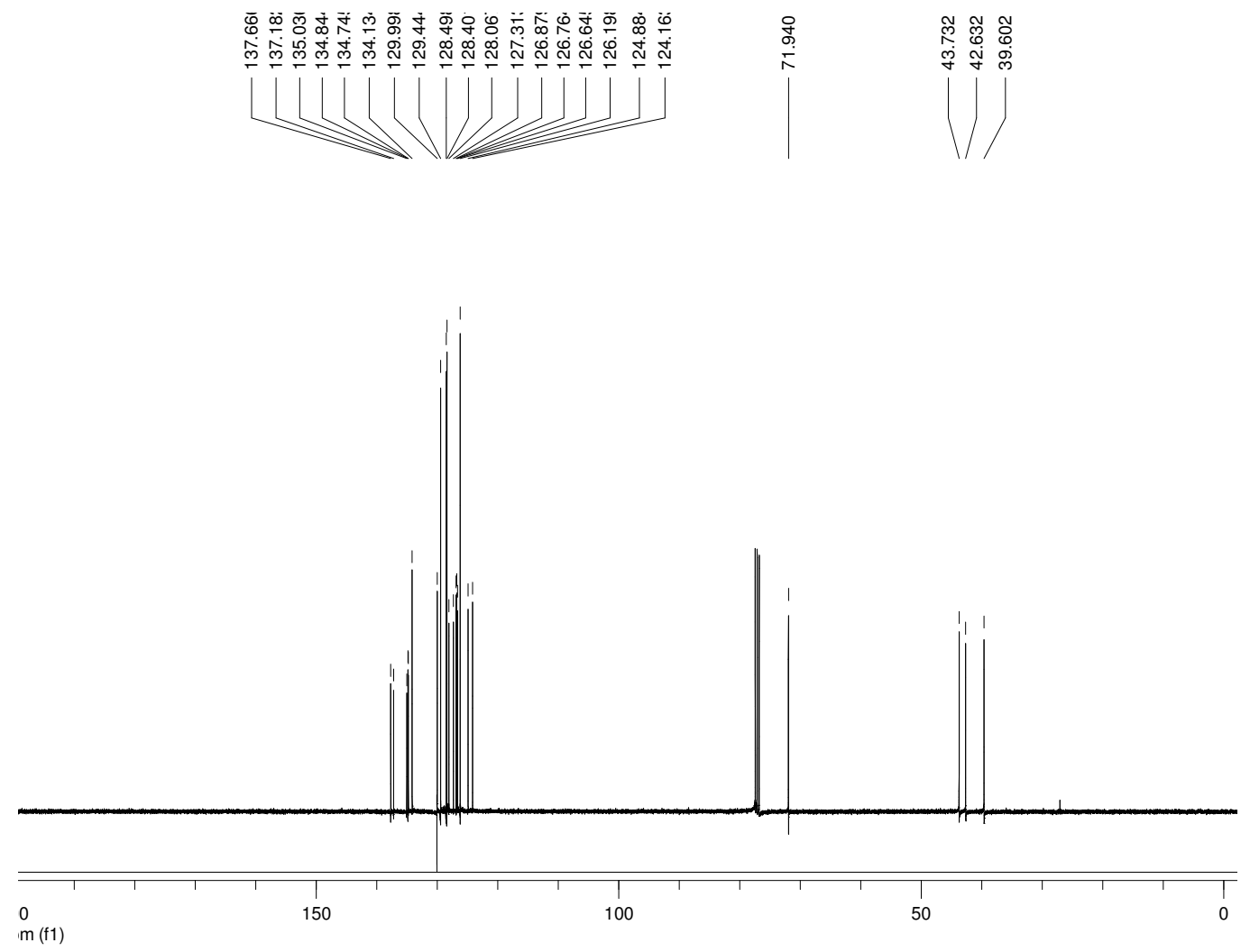


\section{$(E, E)-\mathbf{1 1}\left({ }^{1} \mathrm{H}\right):$}

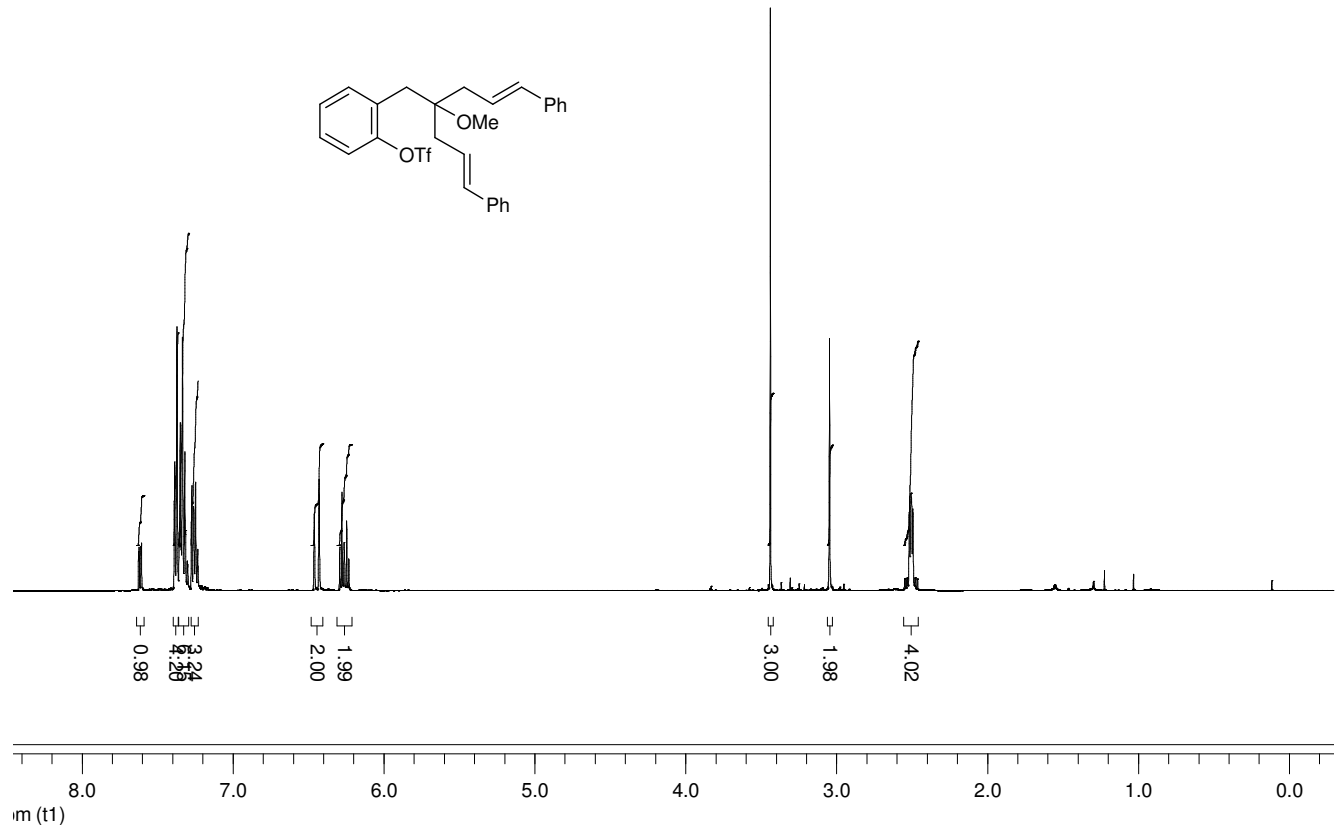

$(E, E)-11\left({ }^{13} \mathrm{C}\right):$

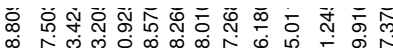

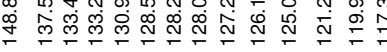
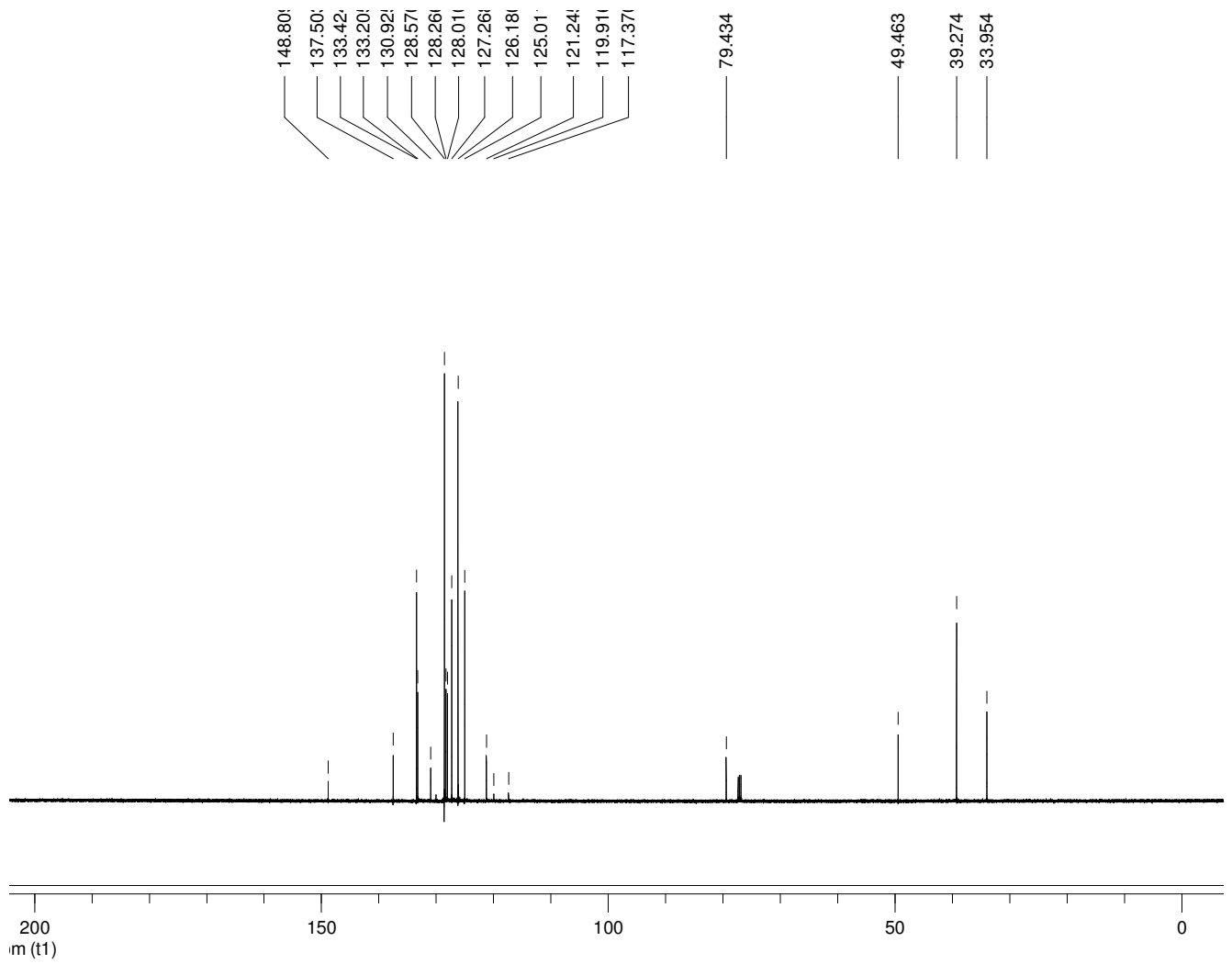
$(R, E, E)-\mathbf{1 5}\left({ }^{1} \mathrm{H}\right)$ :

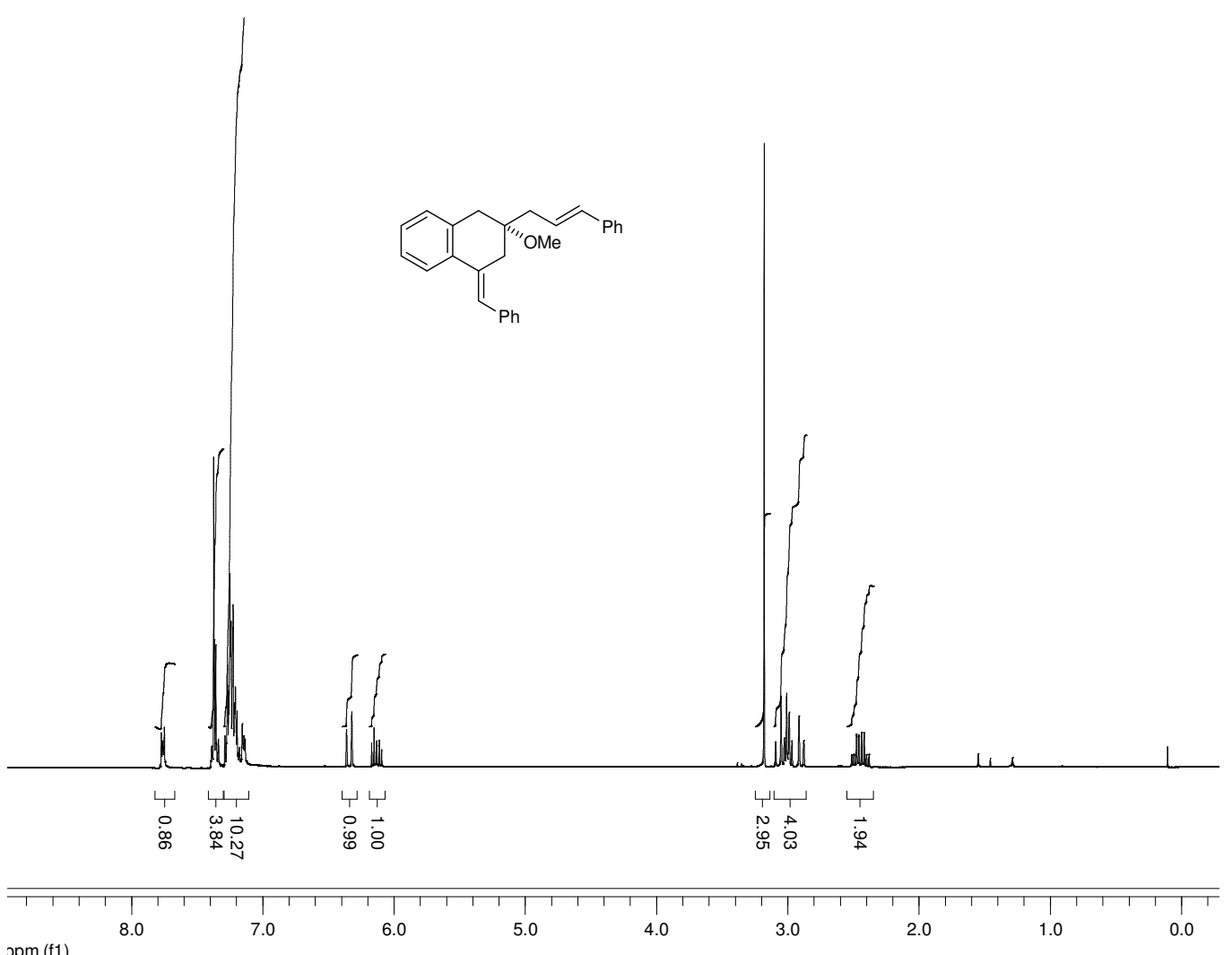

opm (f1)

$(R, E, E)-15\left({ }^{13} \mathrm{C}\right):$
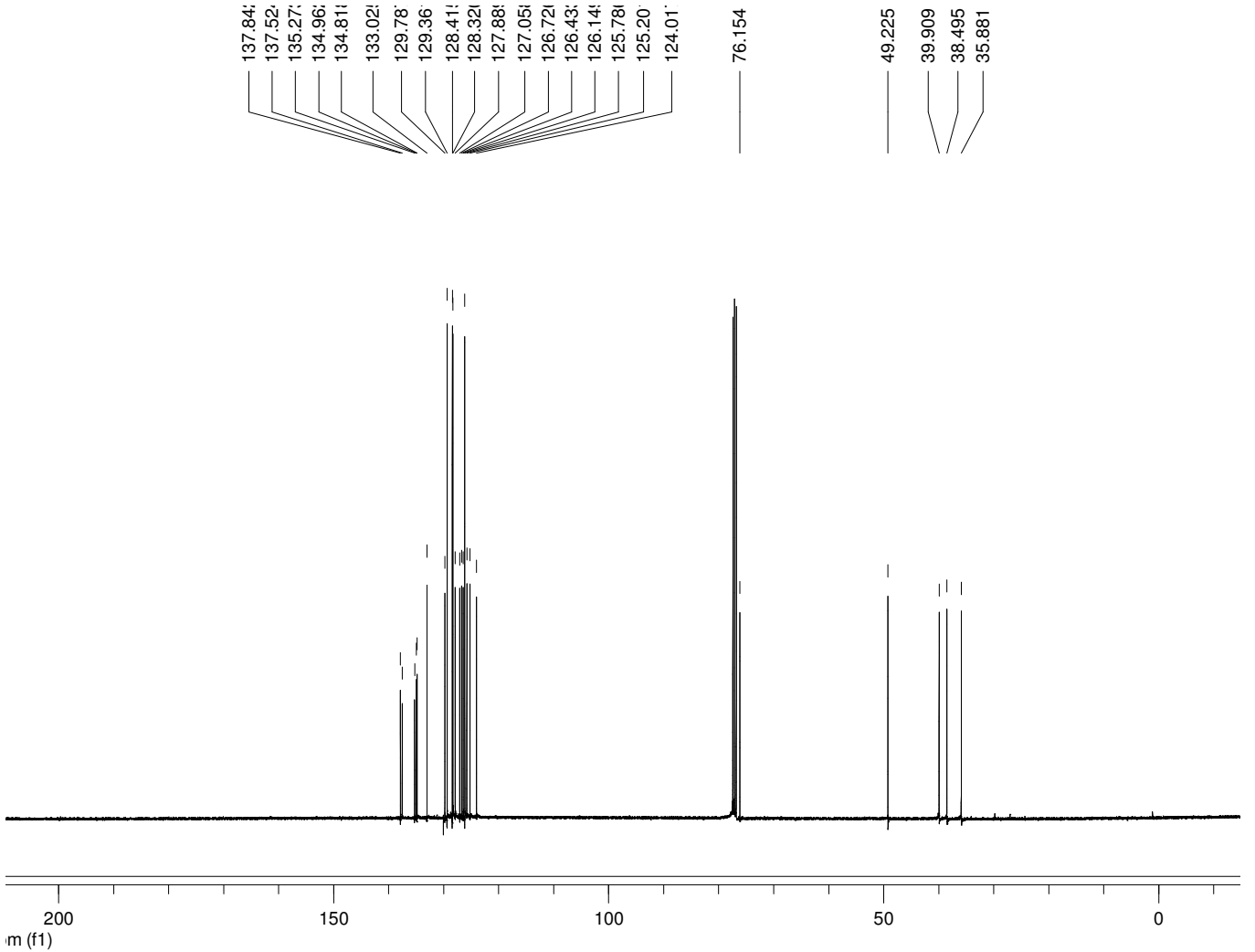


\section{$(E, E)-\mathbf{1 0}\left({ }^{1} \mathrm{H}\right)$ :}

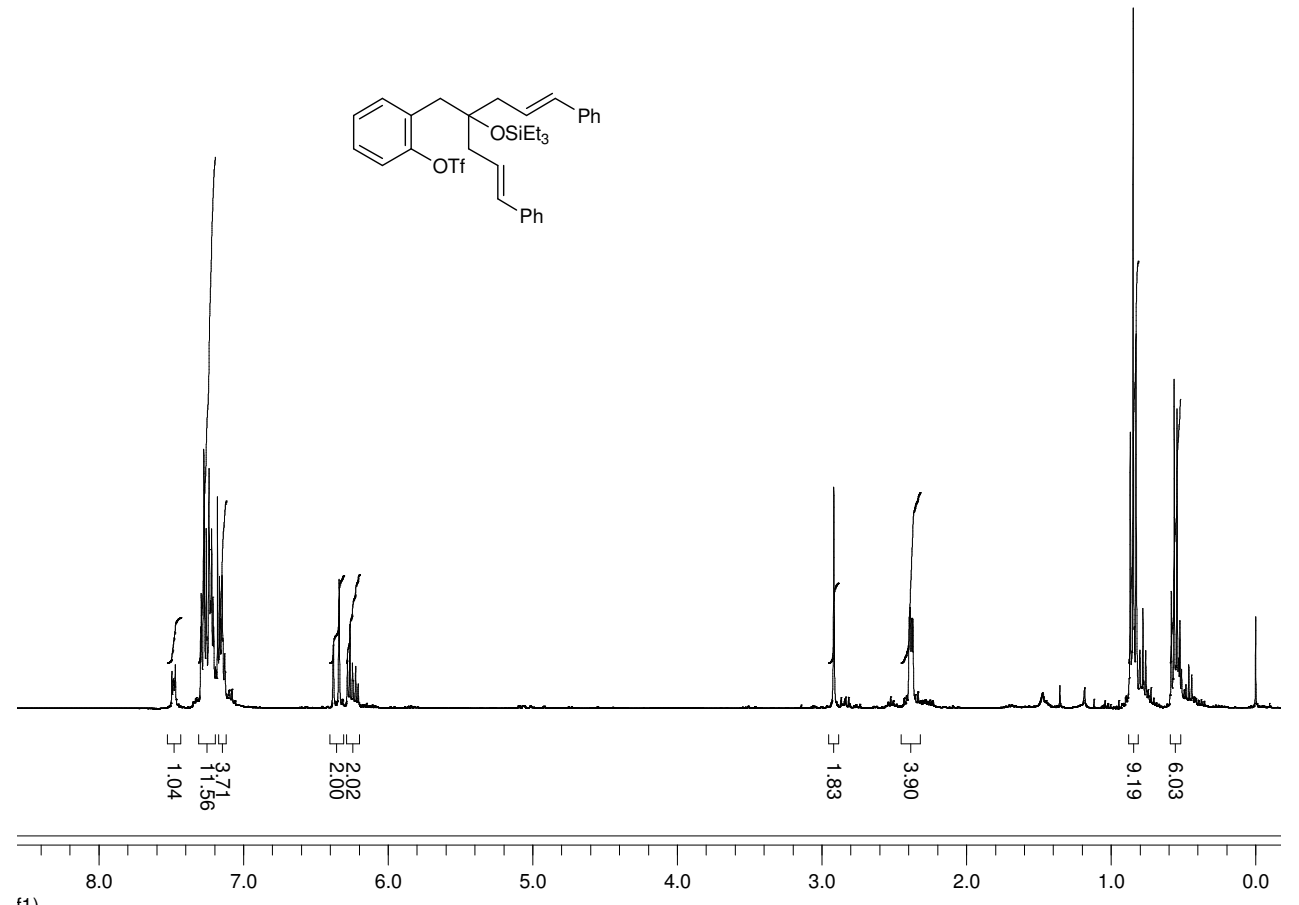

$(E, E)-10\left({ }^{13} \mathrm{C}\right):$

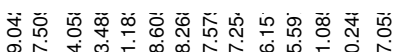

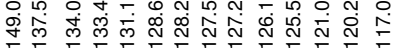
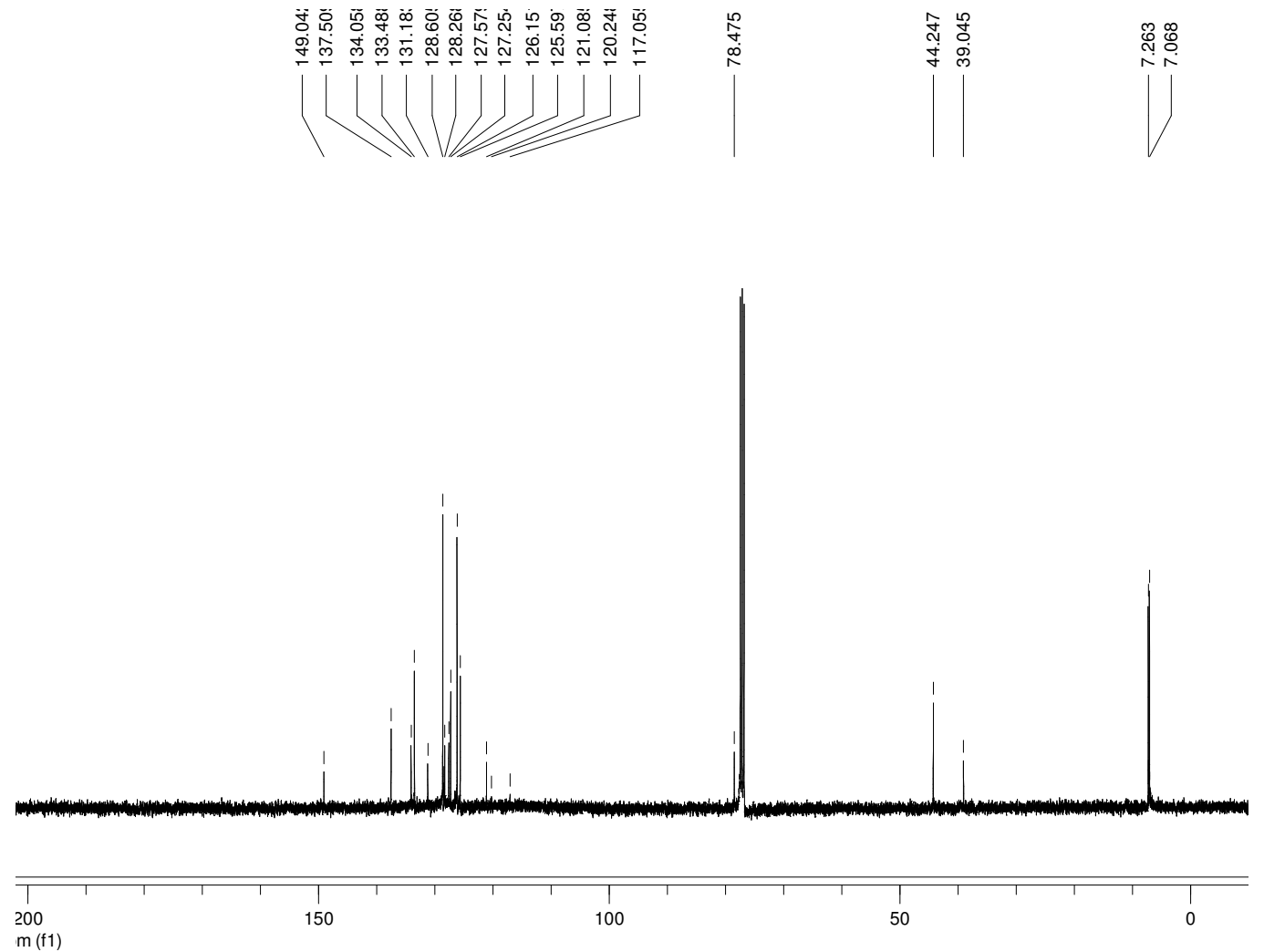
$(R, E, E)-14\left({ }^{1} H\right):$

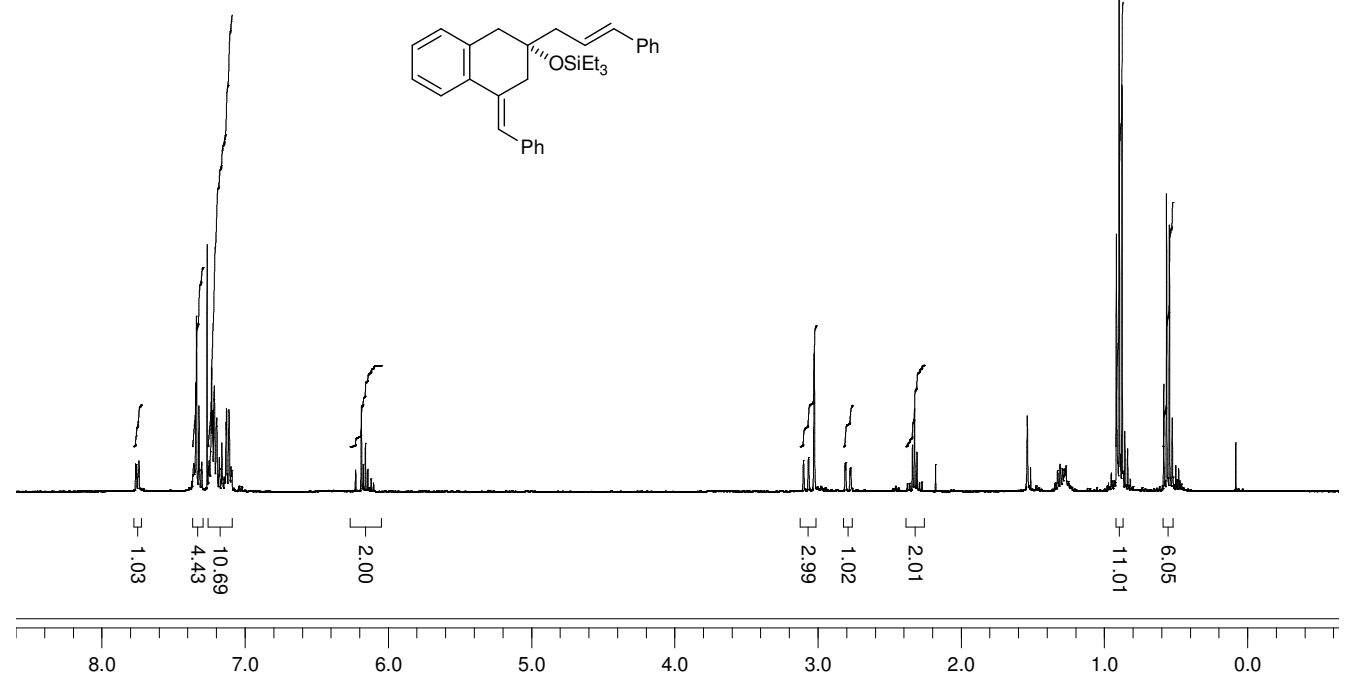

f1)

$(R, E, E)-14\left({ }^{13} \mathrm{C}\right)$ :
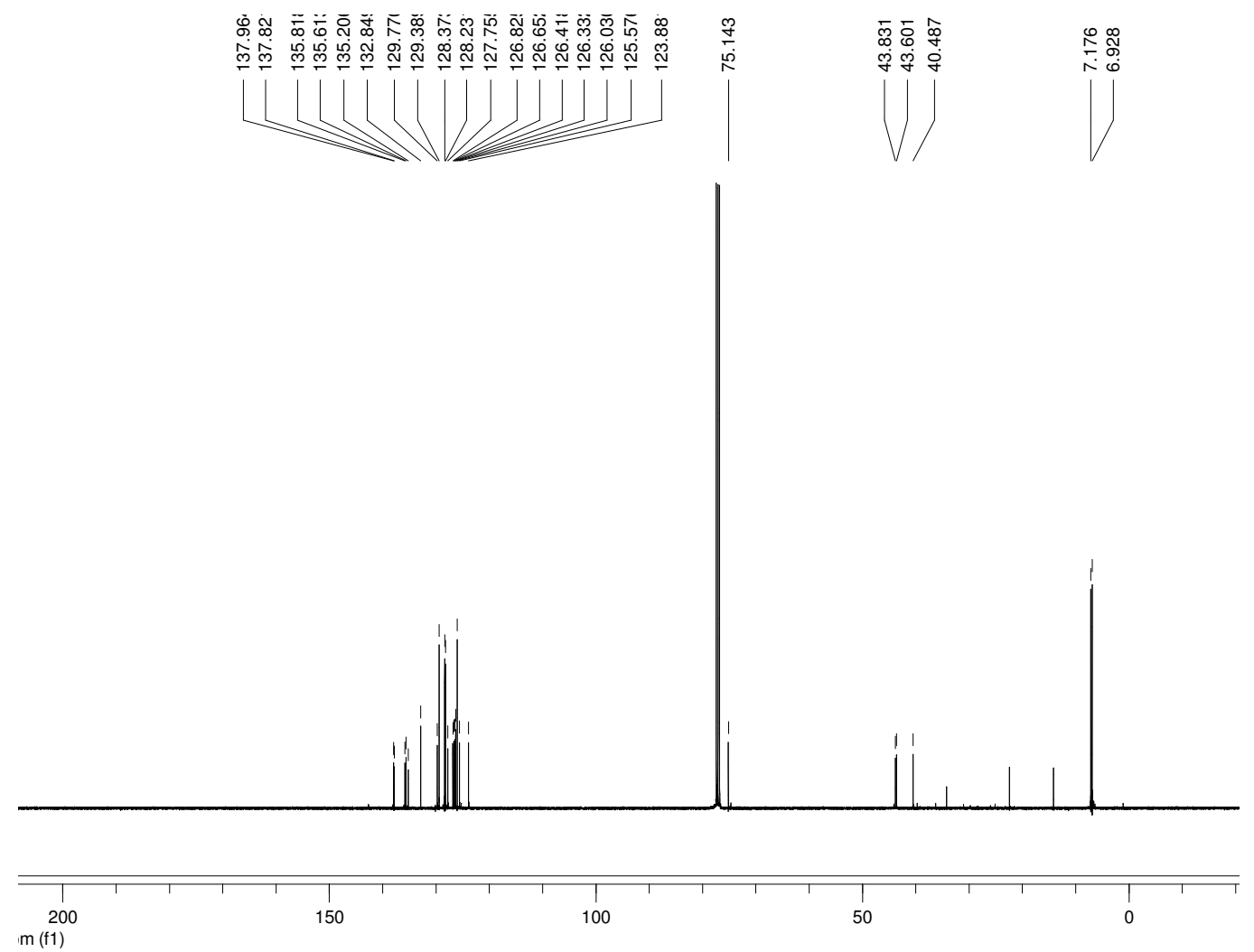
$(E, E)-12\left({ }^{1} H\right)$ :

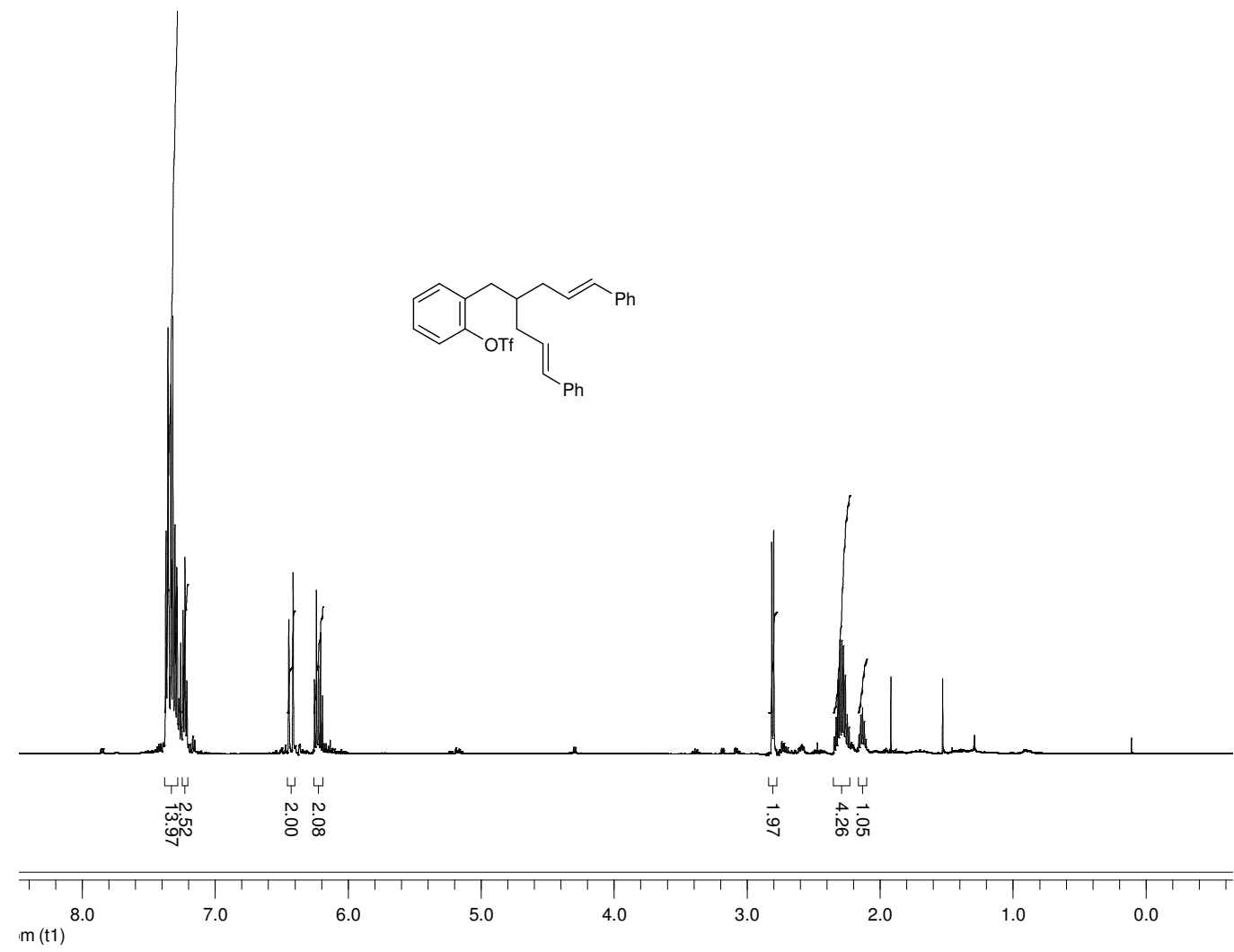

$(E, E)-12\left({ }^{13} \mathrm{C}\right):$

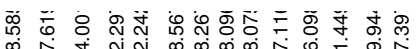

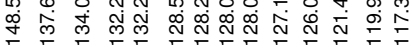

$\lfloor\lfloor\lfloor\mid\rfloor\rfloor\rfloor\rfloor\rfloor$

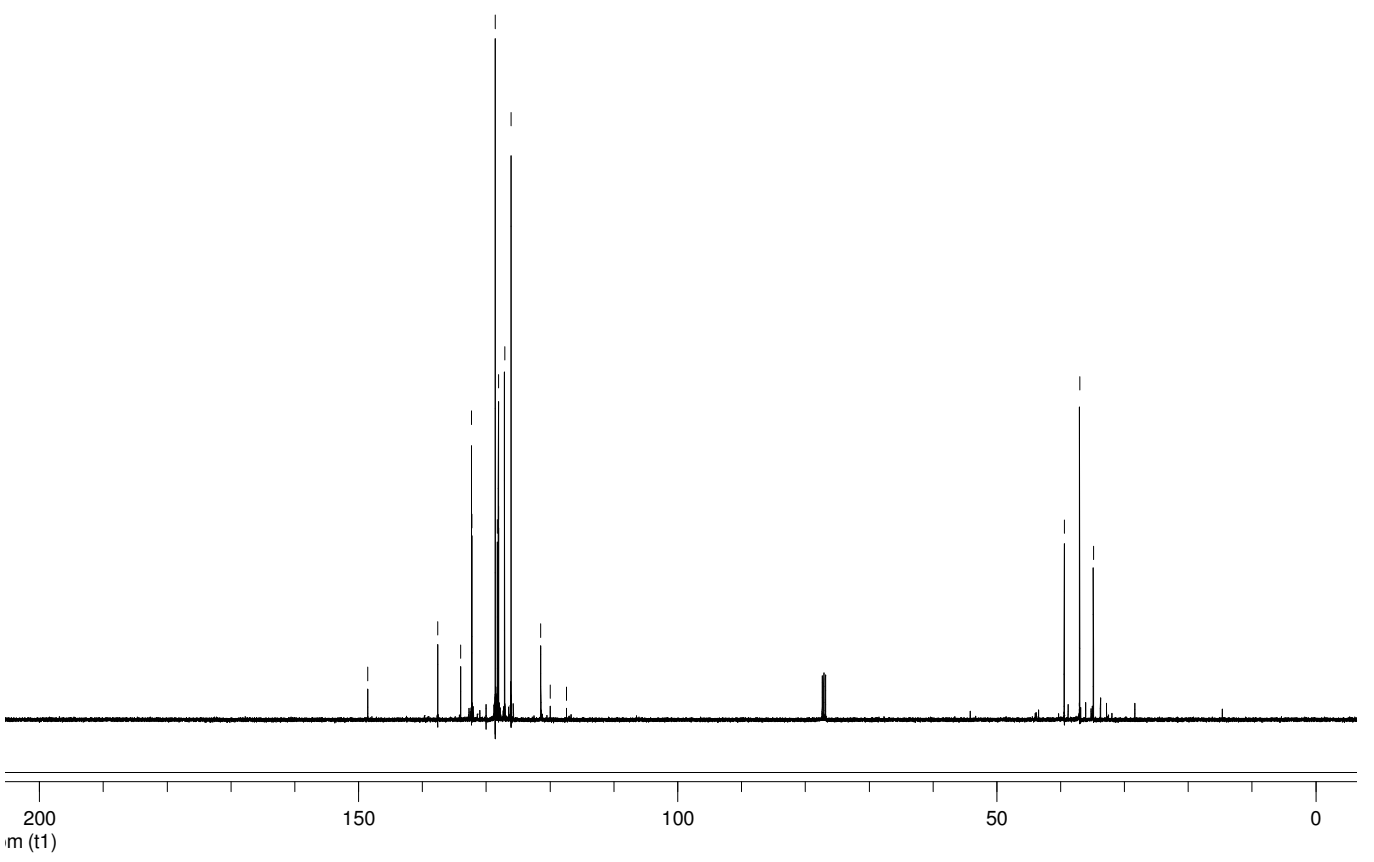


$\left(S^{\star}, E, E\right)-16\left({ }^{1} H\right):$

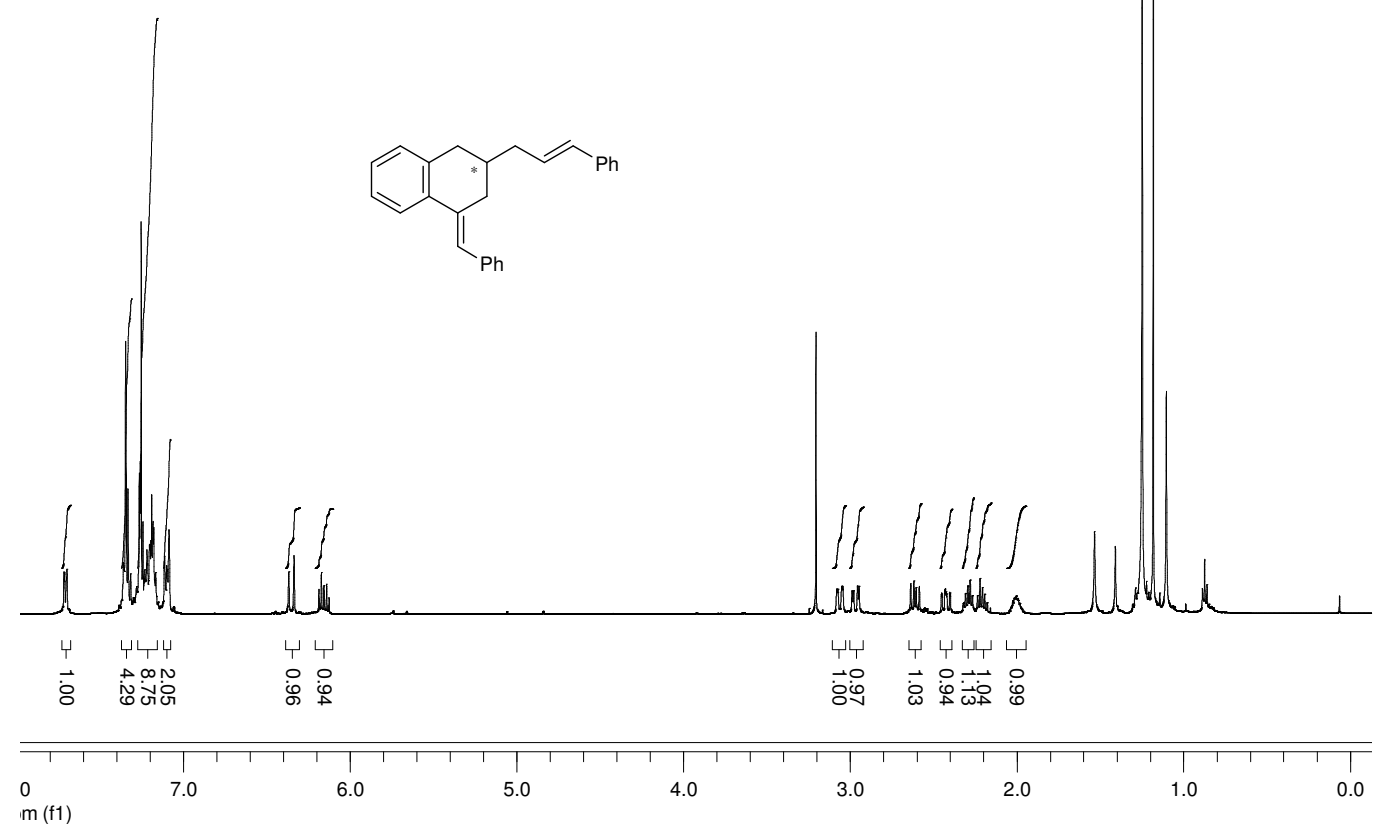

$\left(S^{\star}, E, E\right)-16\left({ }^{13} \mathrm{C}\right):$

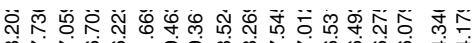

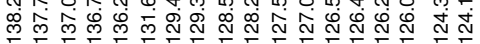

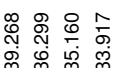
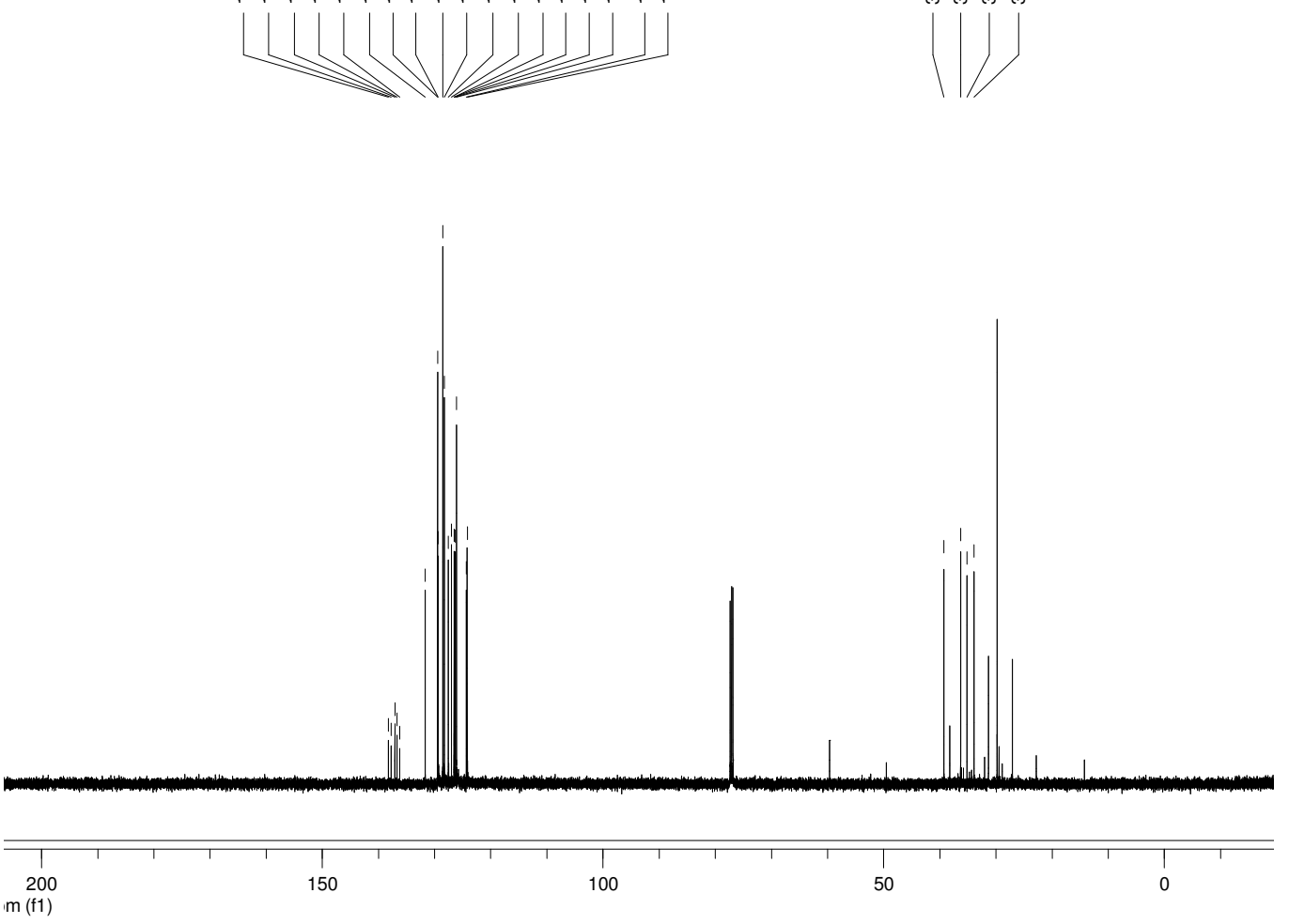
$(E, E)-7\left({ }^{1} \mathrm{H}\right)$ :

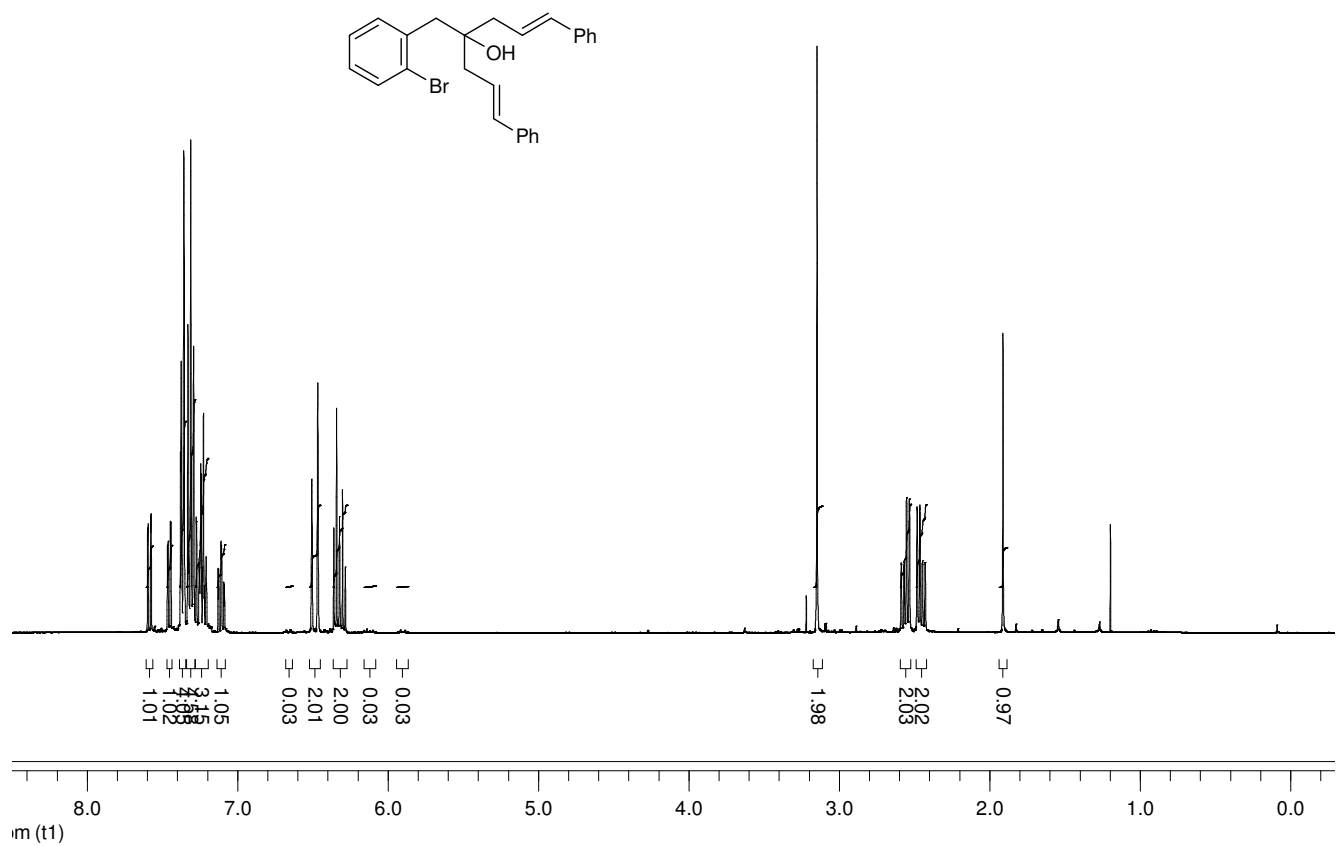

$(E, E)-7\left({ }^{13} \mathrm{C}\right)$ :

कृ

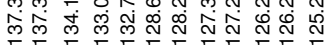

LULUJj
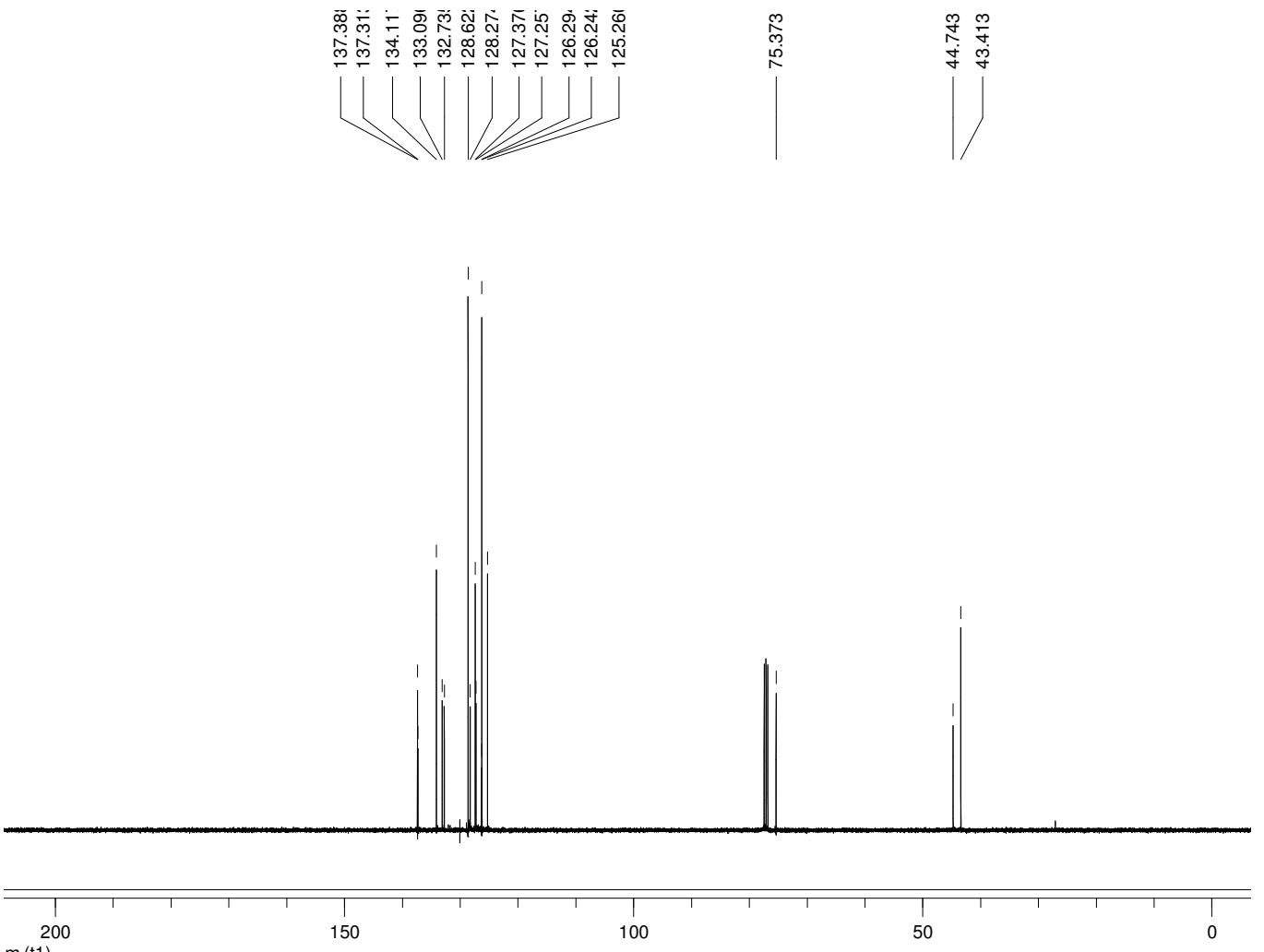

m(t1) 


\section{$(E, E)-8\left({ }^{1} \mathrm{H}\right):$}

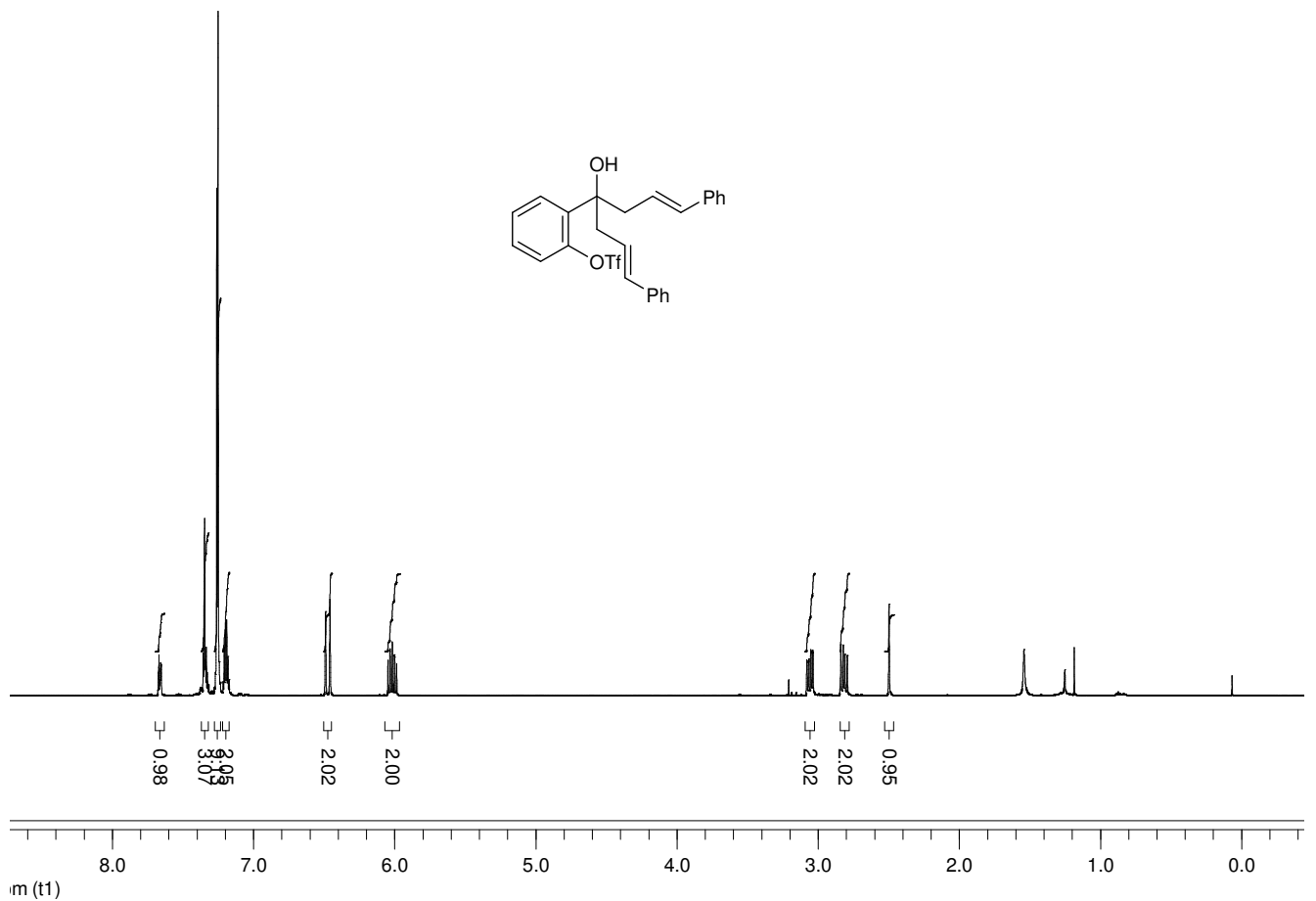

\section{$(E, E)-8\left({ }^{13} C\right):$}
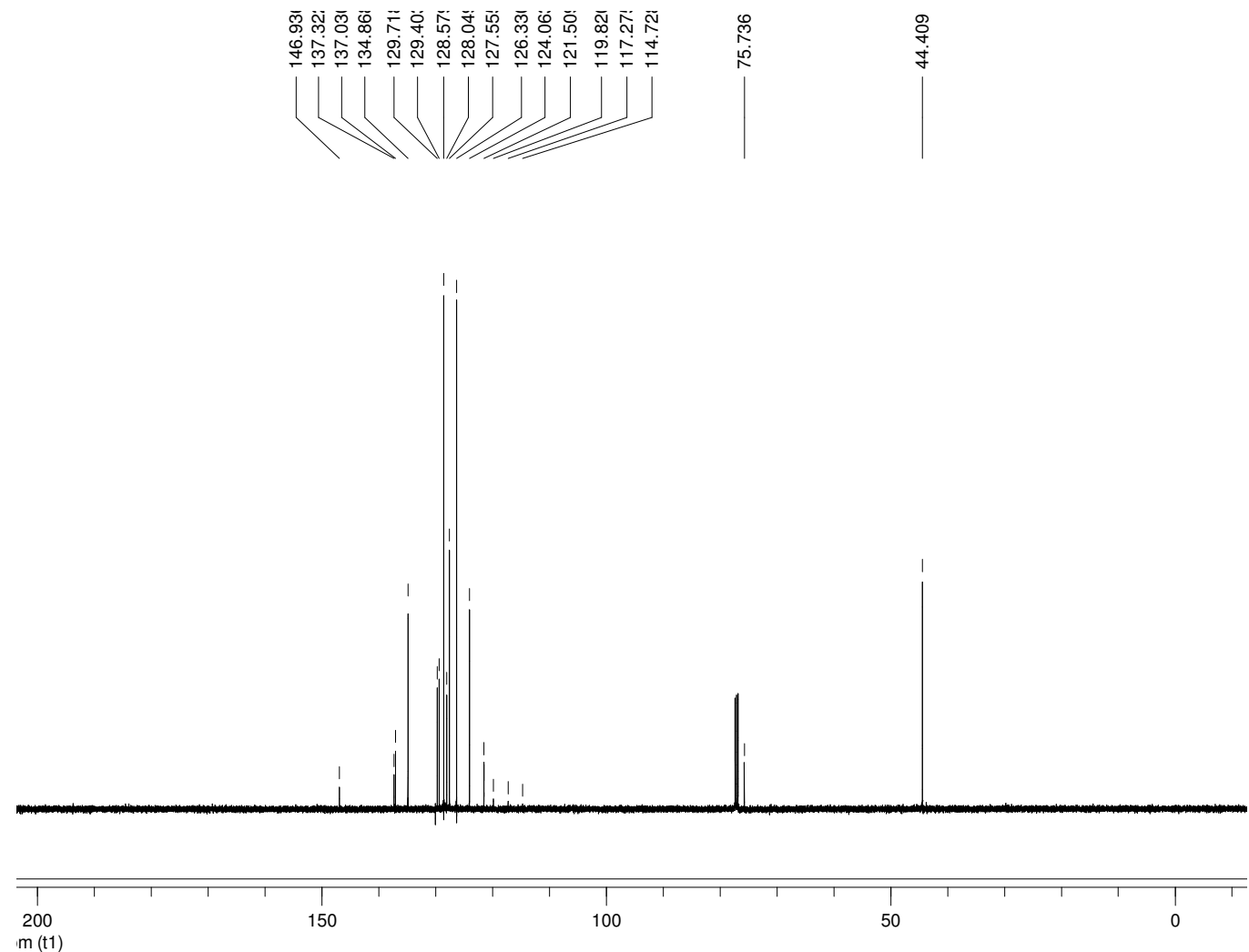
$\left(R^{\star}, E, E\right)-17\left({ }^{1} H\right)$ :

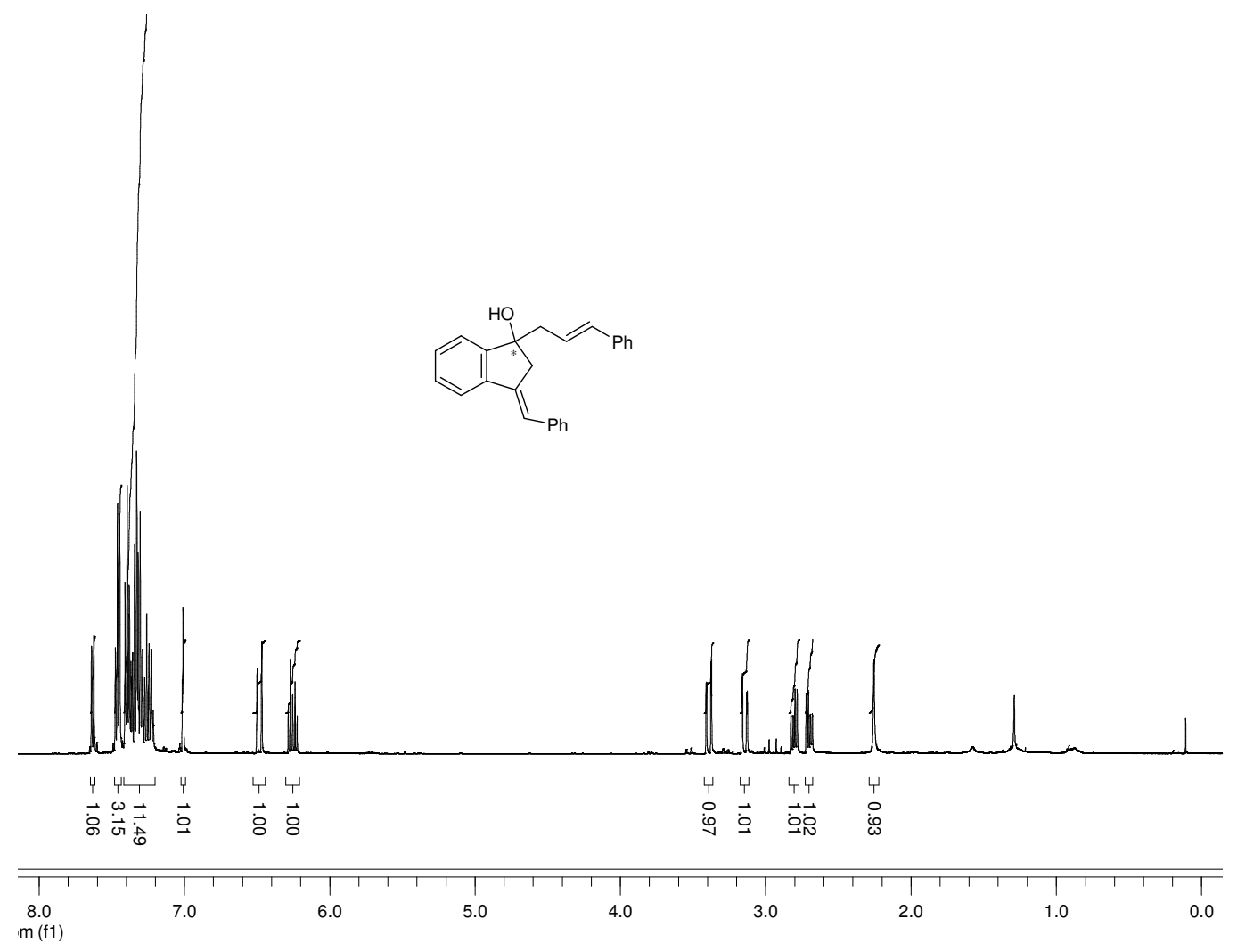

$\left(R^{\star}, E, E\right)-17\left({ }^{13} \mathrm{C}\right):$
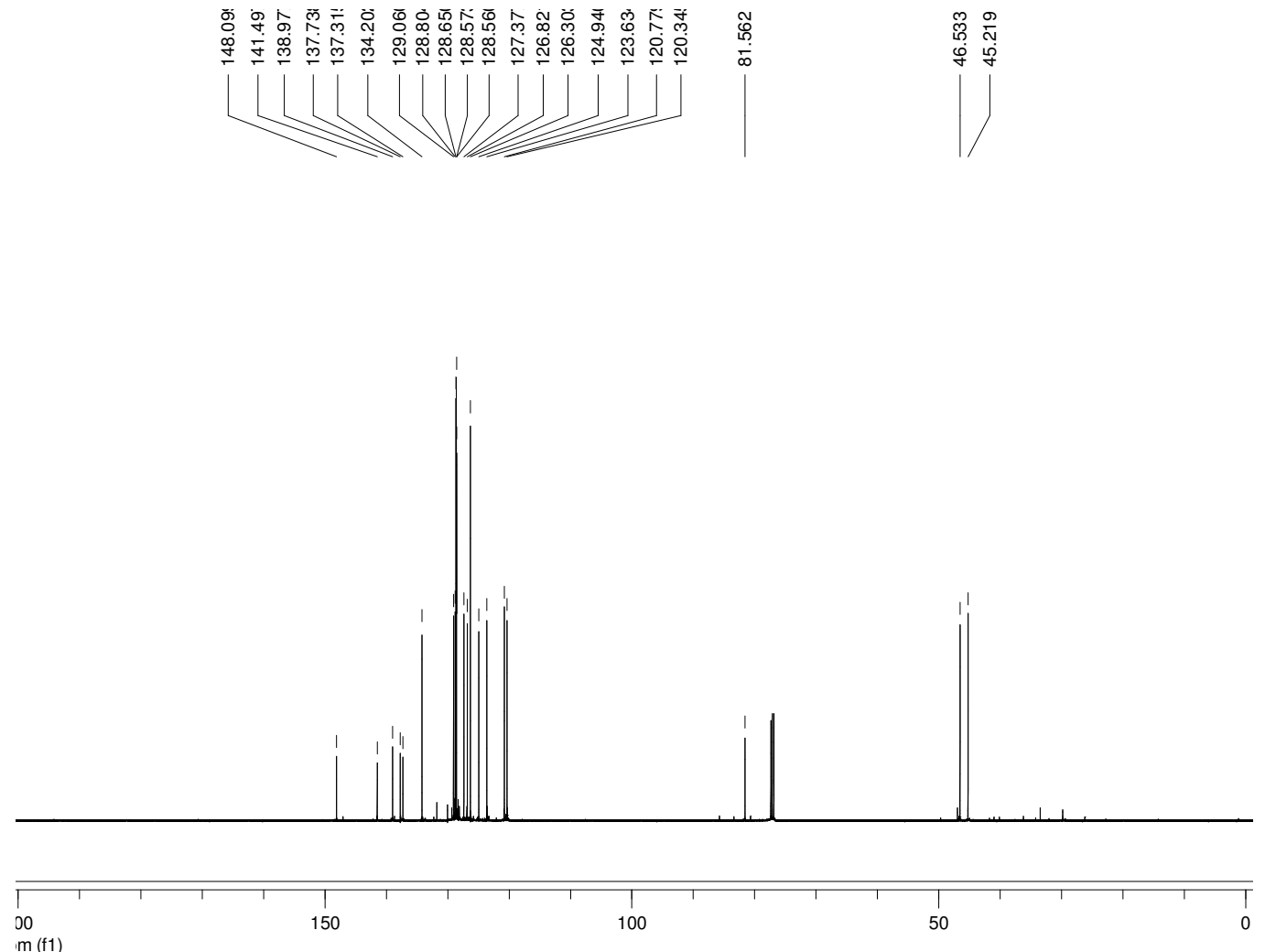
$(Z, Z)-9\left({ }^{1} H\right)$ :

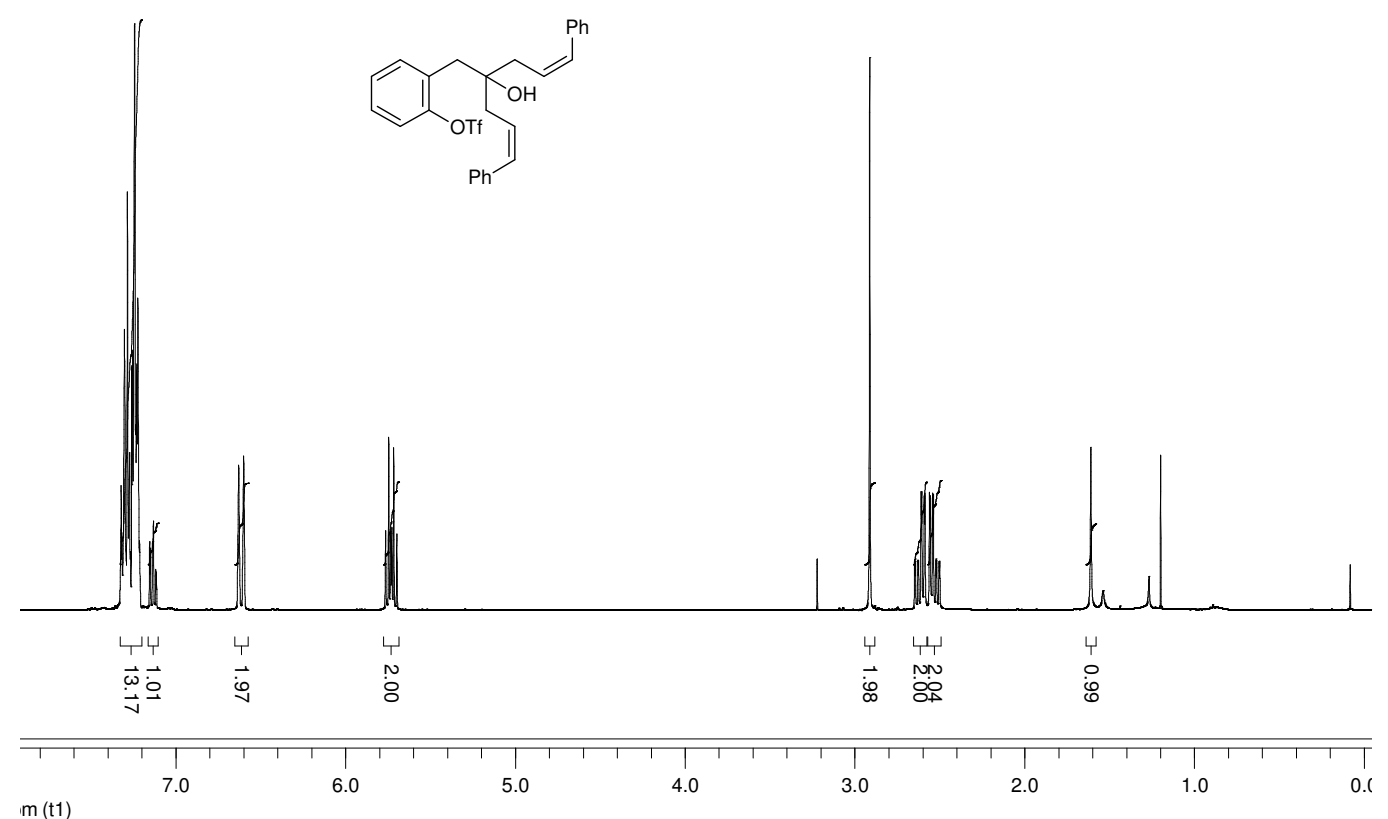

$(Z, Z)-9\left({ }^{13} \mathrm{C}\right):$
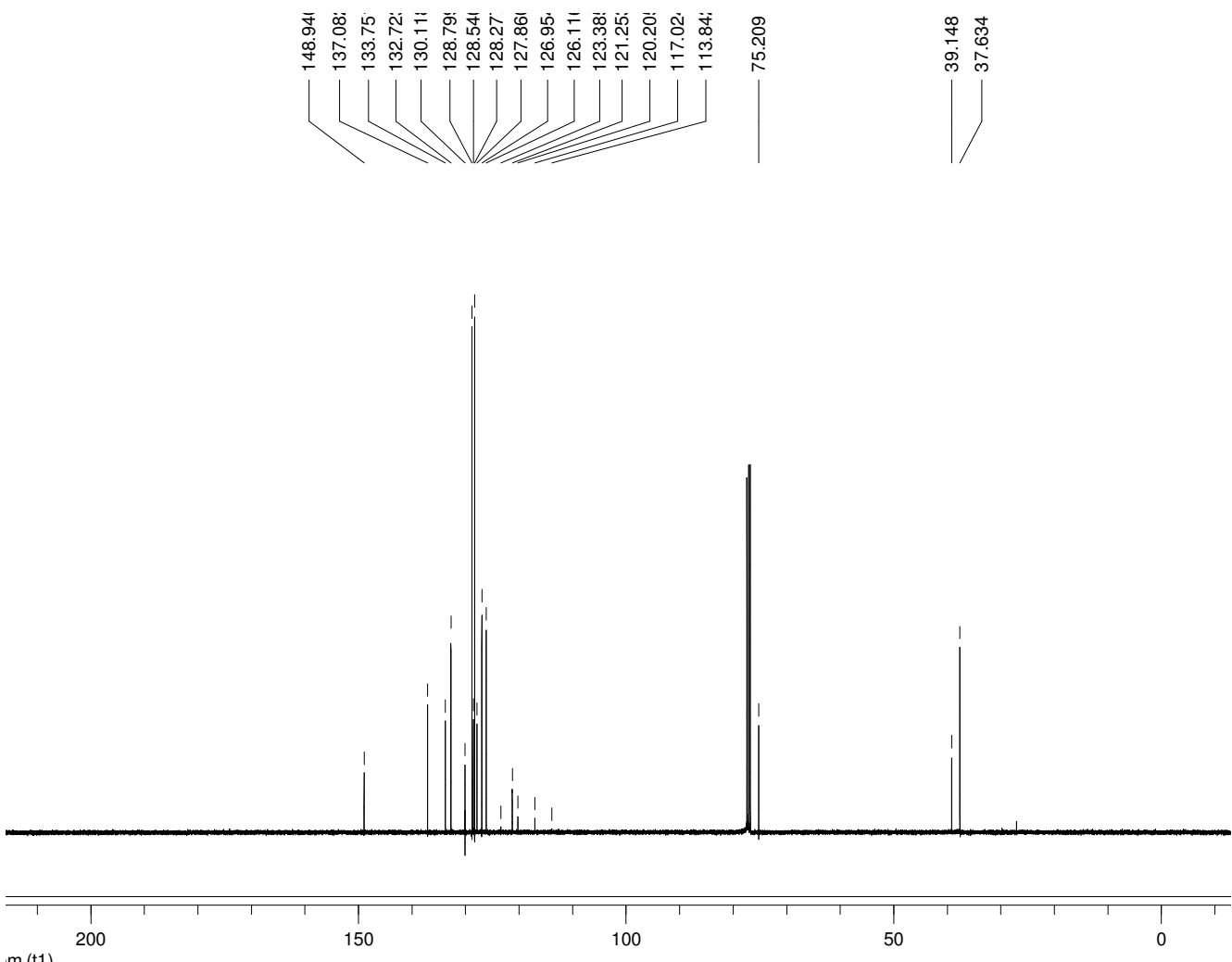

m (t1) 
$(R, Z, Z)-\mathbf{1 3}\left({ }^{1} \mathrm{H}\right):$

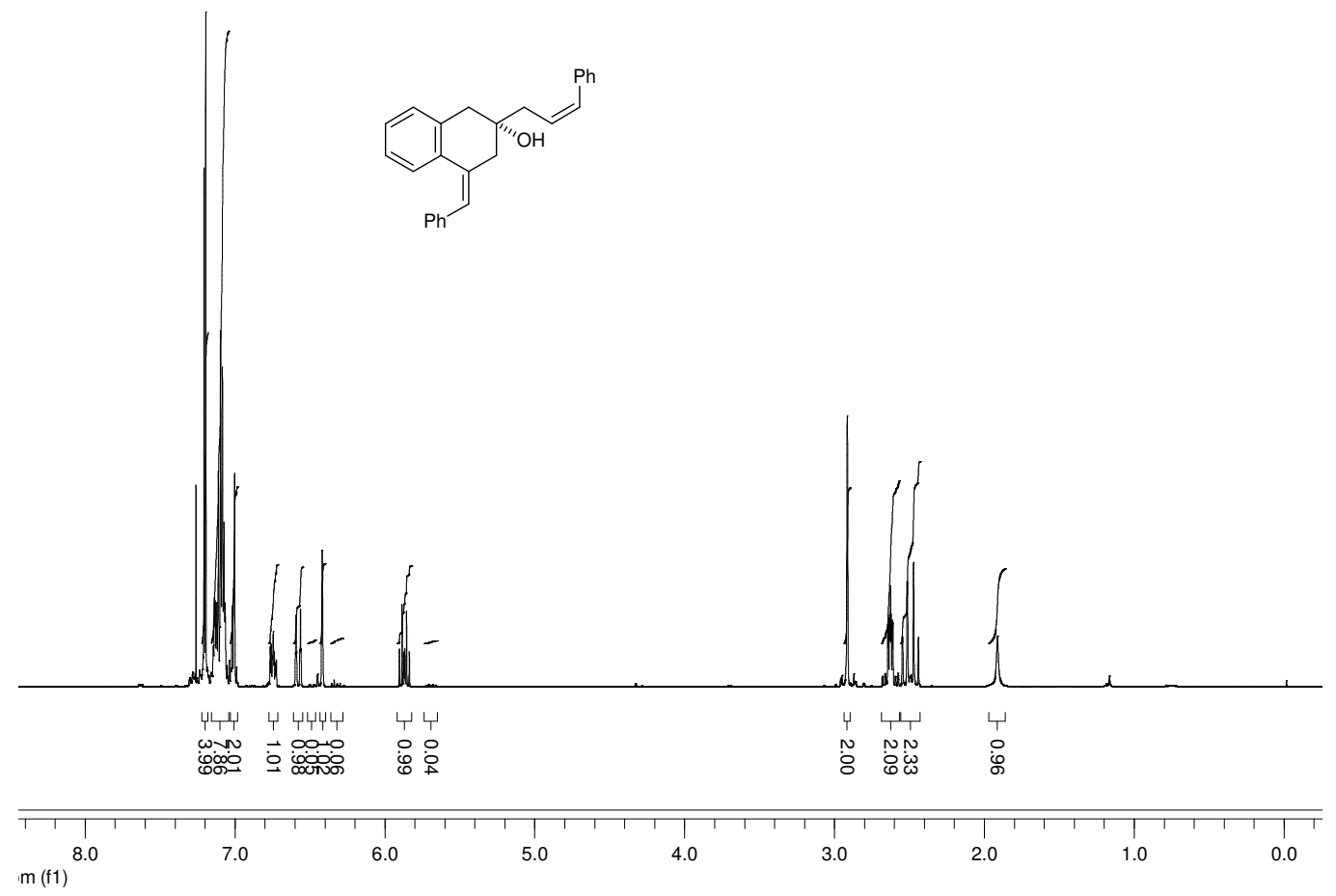

$(R, Z, Z)-13\left({ }^{13} \mathrm{C}\right):$

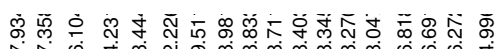

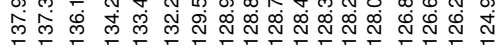
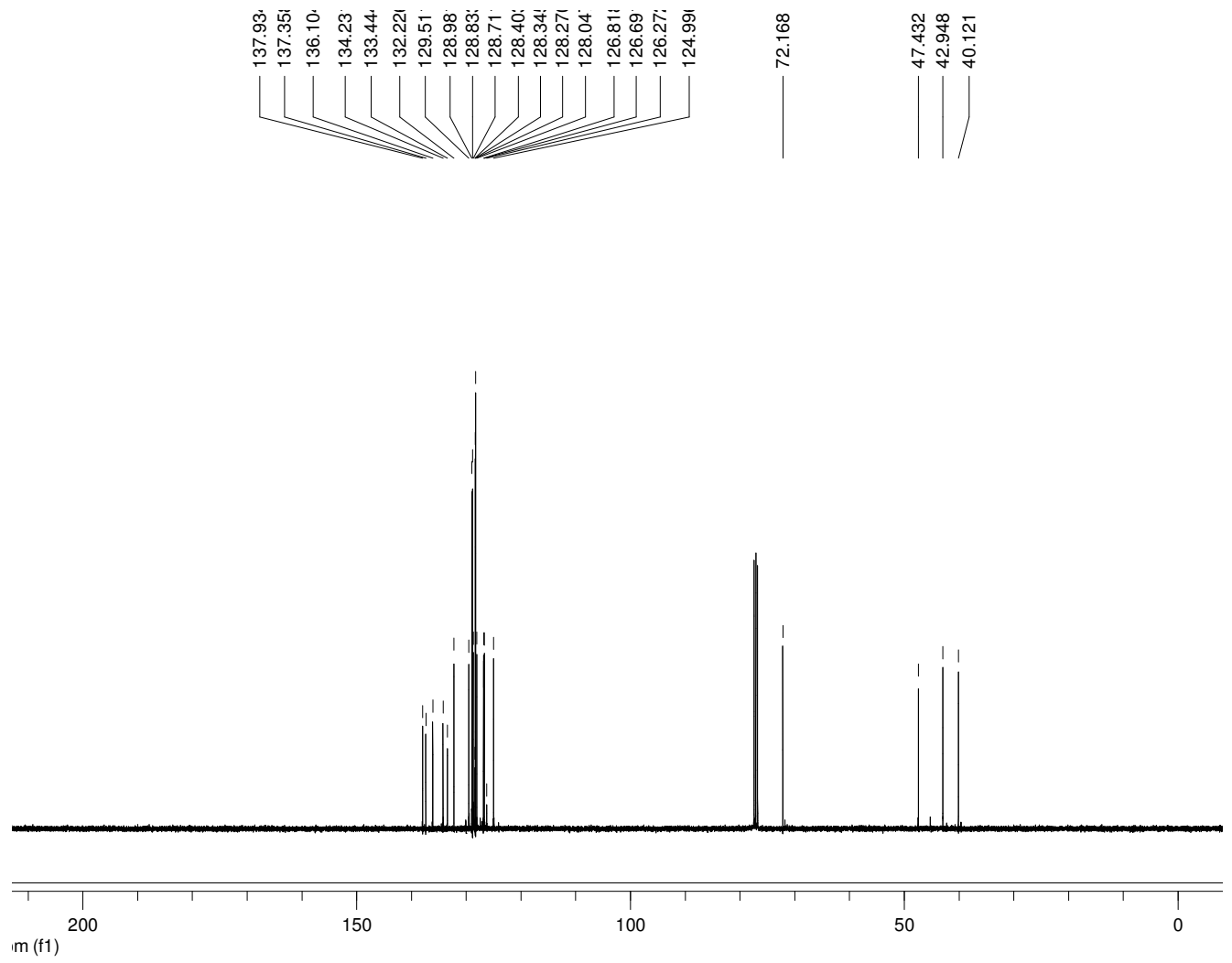
$(Z, Z)-11\left({ }^{1} H\right):$
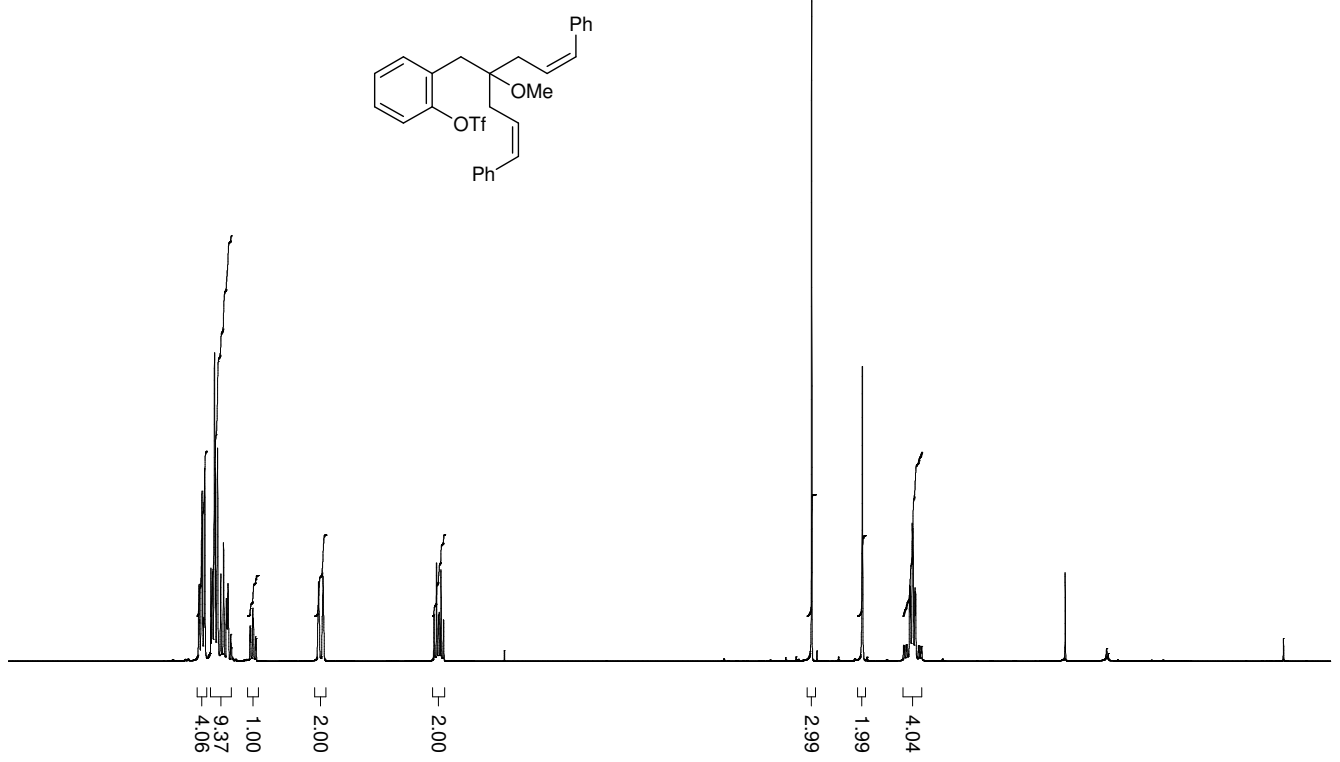

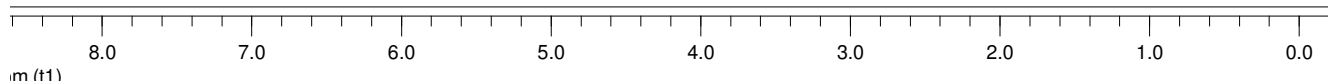

$(Z, Z)-11\left({ }^{13} \mathrm{C}\right):$

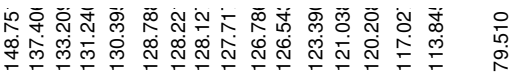
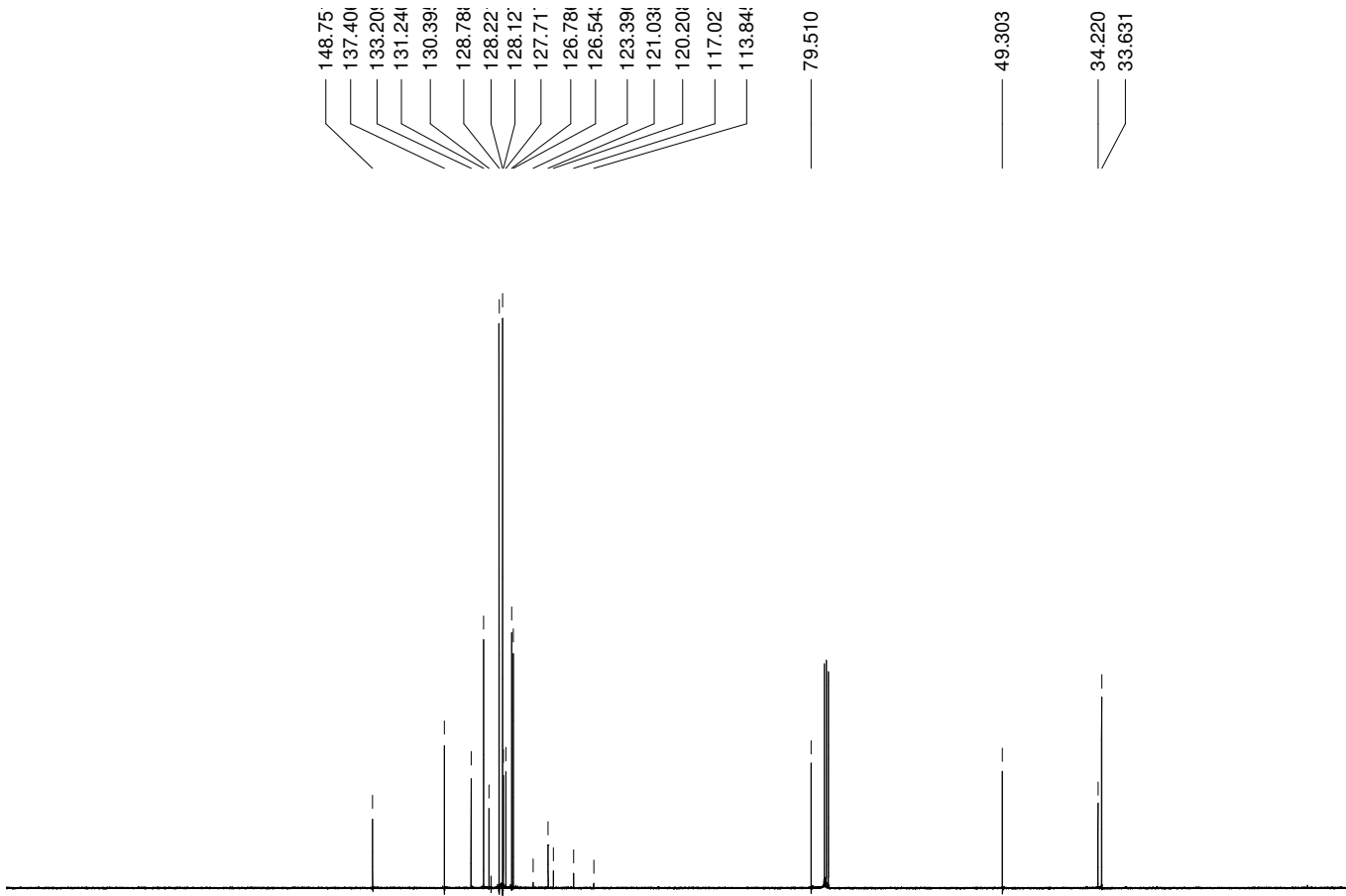

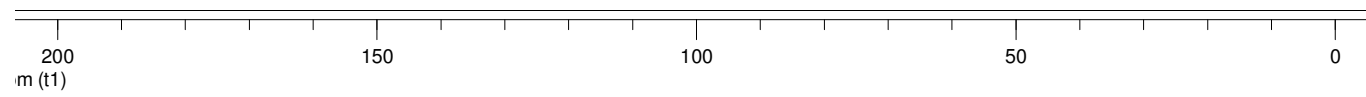


$(R, Z, Z)-15\left({ }^{1} \mathrm{H}\right):$

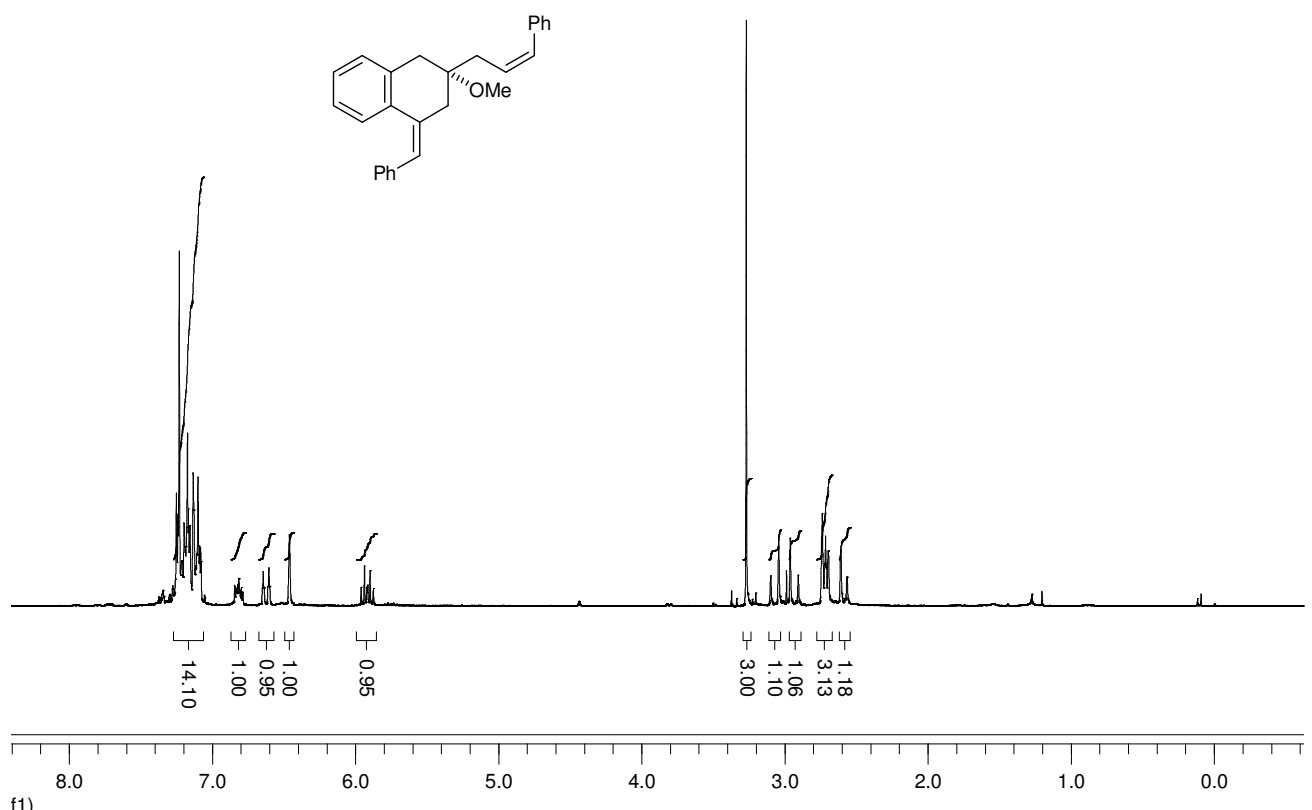

f1)

$(R, Z, Z)-15\left({ }^{13} \mathrm{C}\right):$

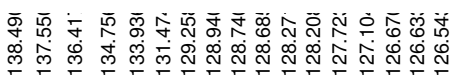
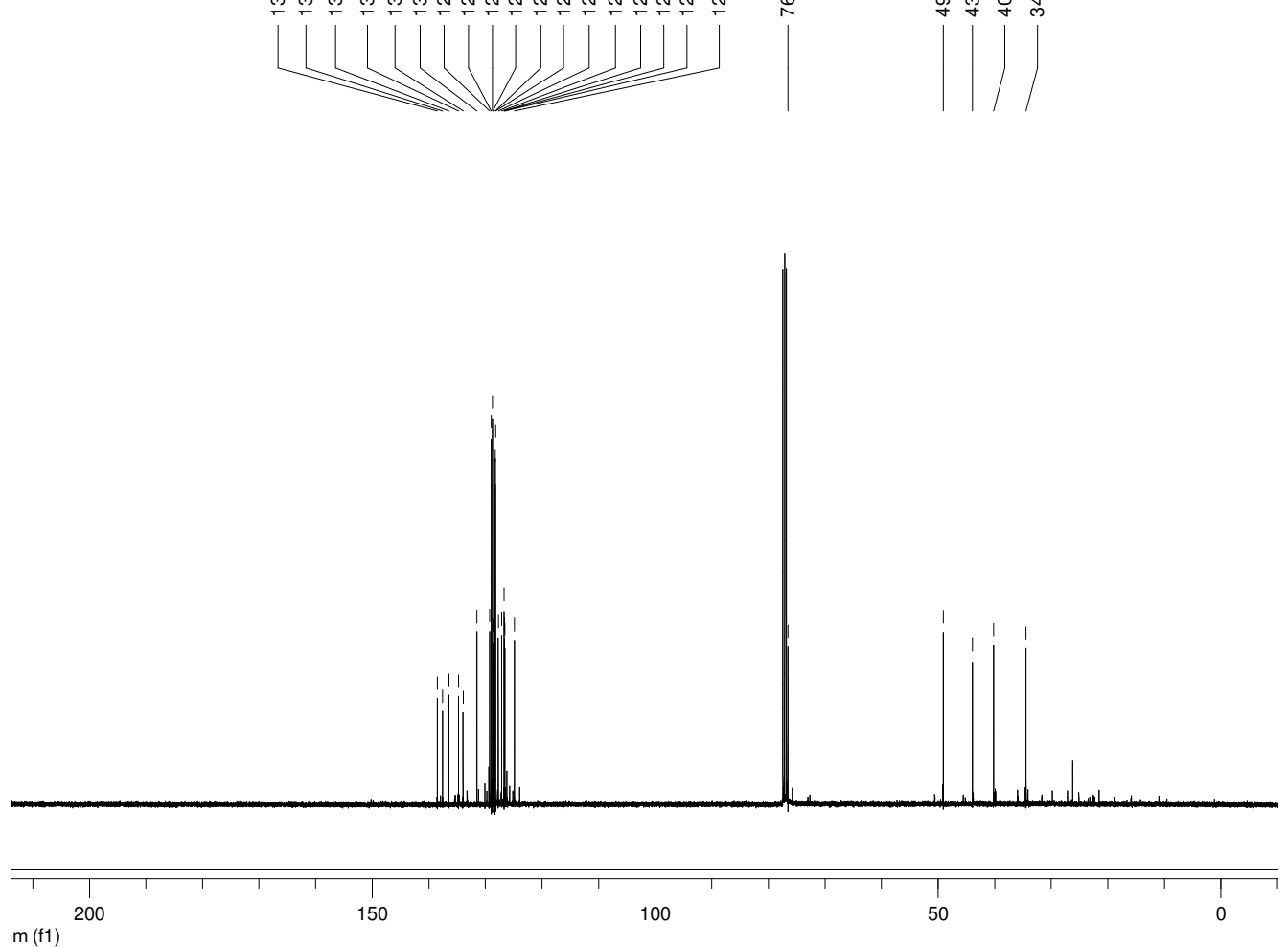


\section{Copies of NMR Spectra of Synthetic Intermediates}

SI1 $\left({ }^{1} H\right)$ :

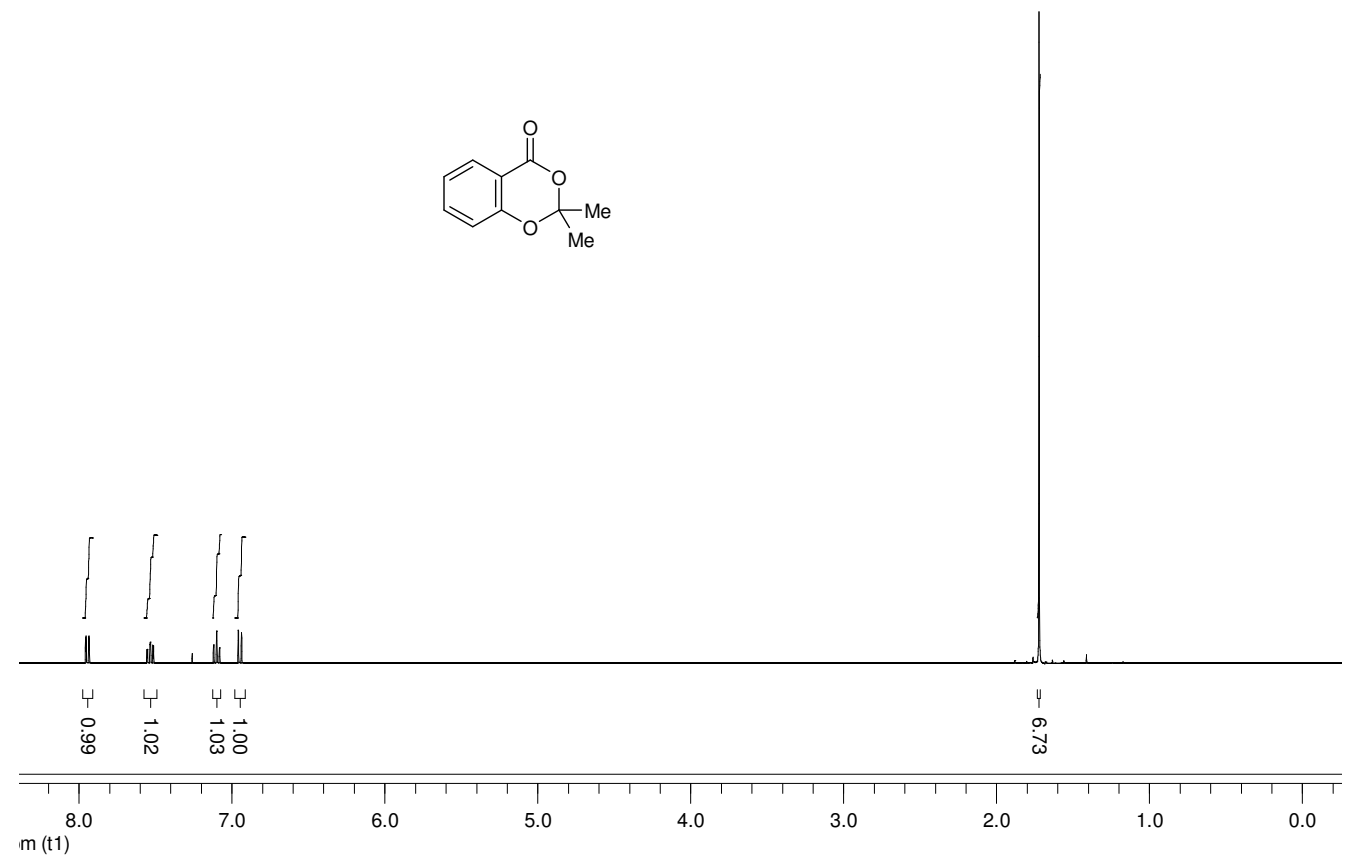

SI1 $\left({ }^{13} \mathrm{C}\right)$ :
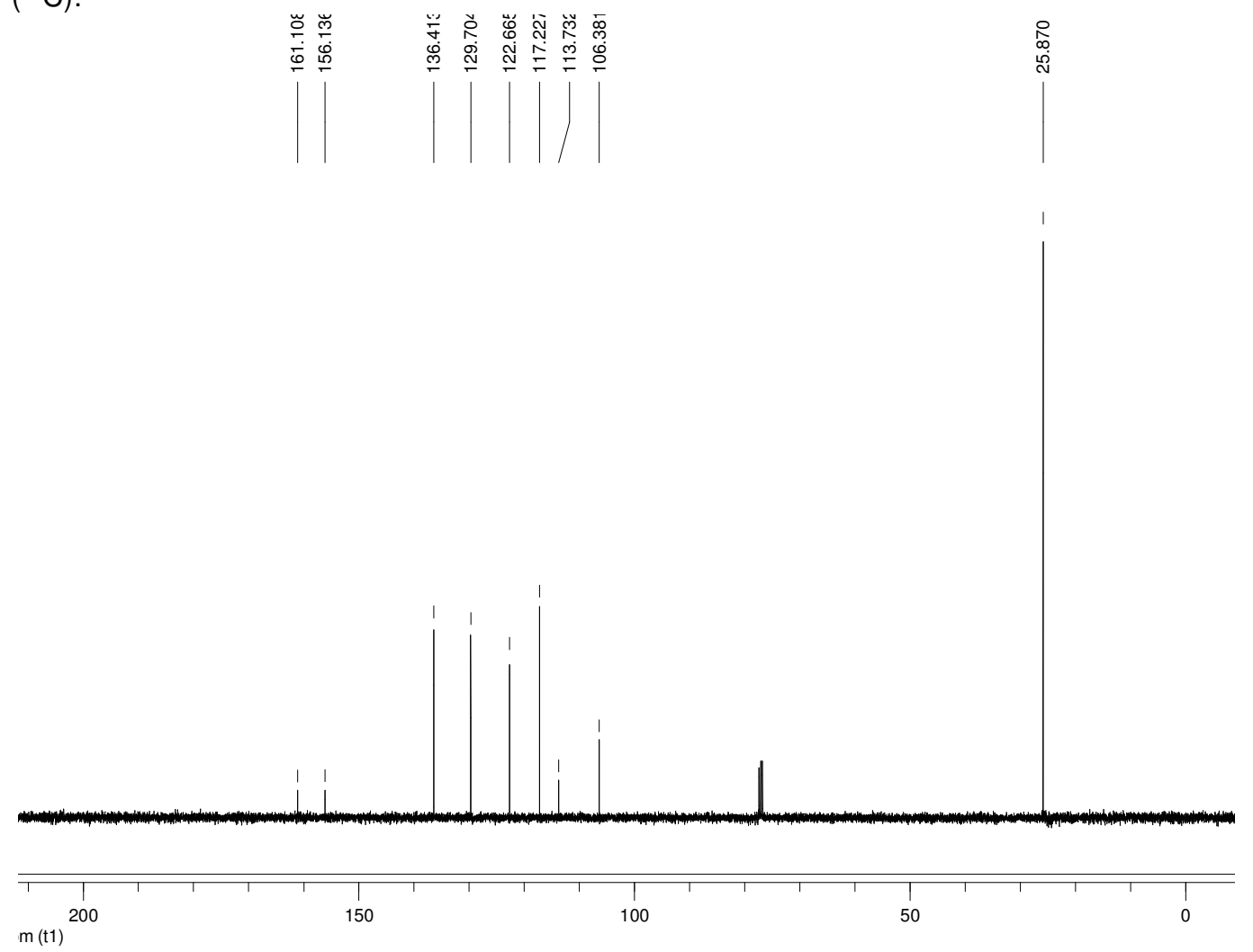
SI5 $\left({ }^{1} \mathrm{H}\right)$ :

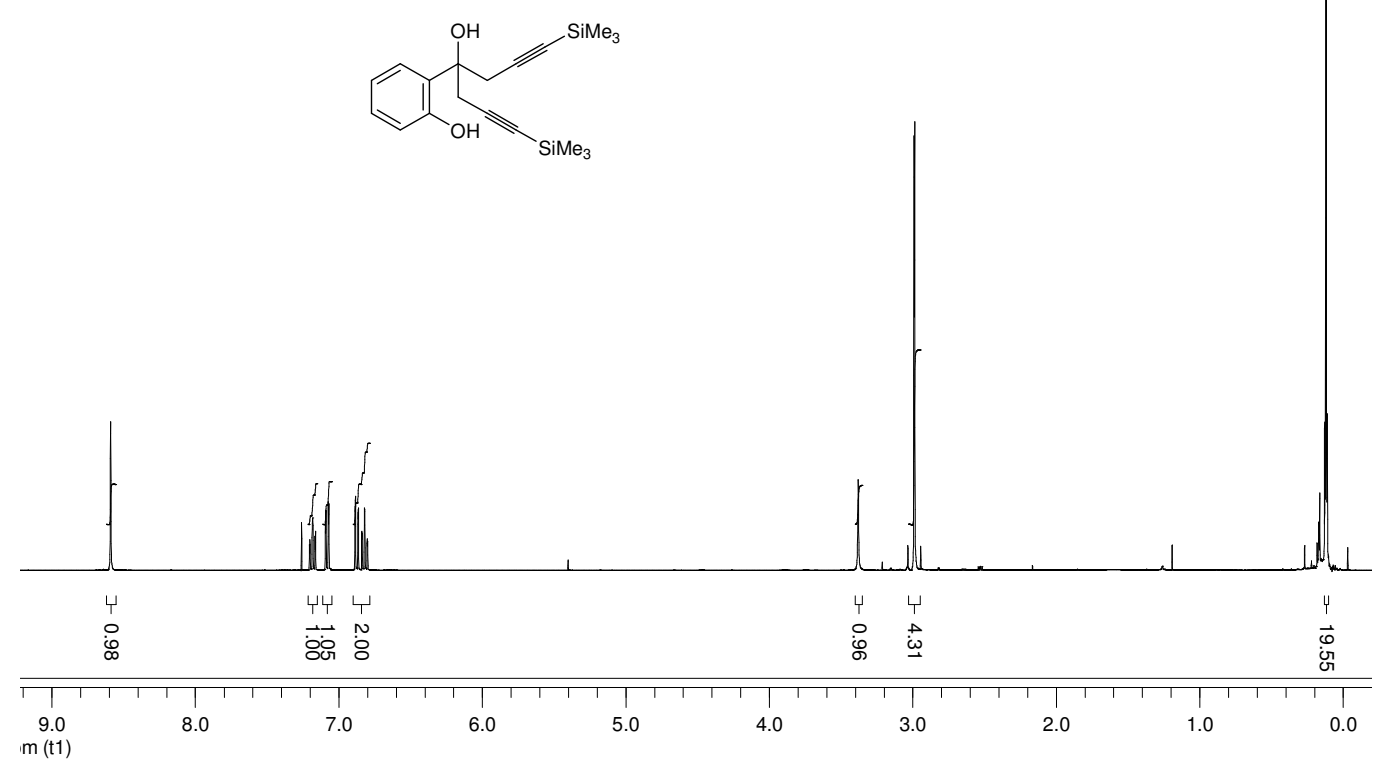

SI5 $\left({ }^{13} \mathrm{C}\right)$ :
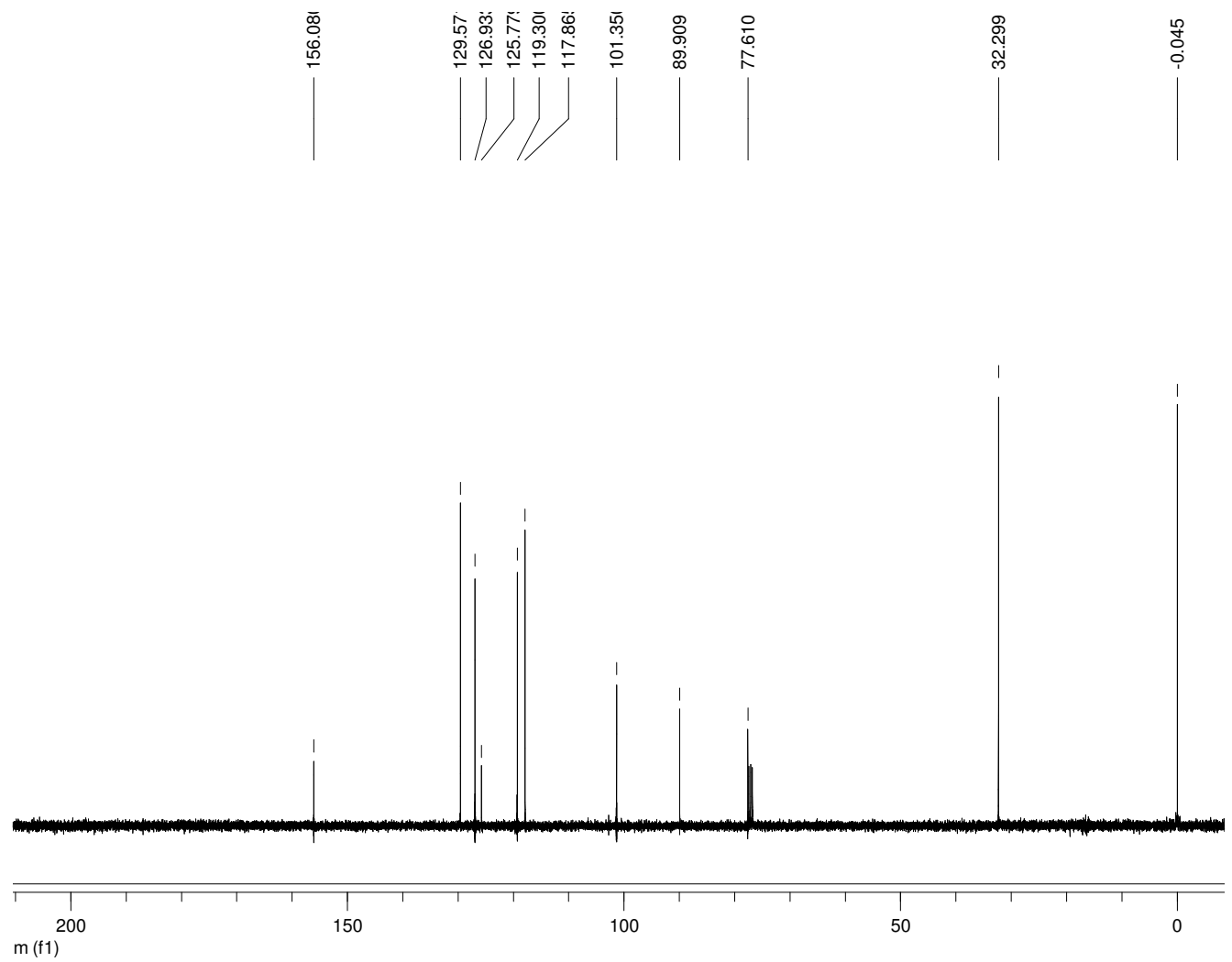
SI6 $\left({ }^{1} H\right)$ :
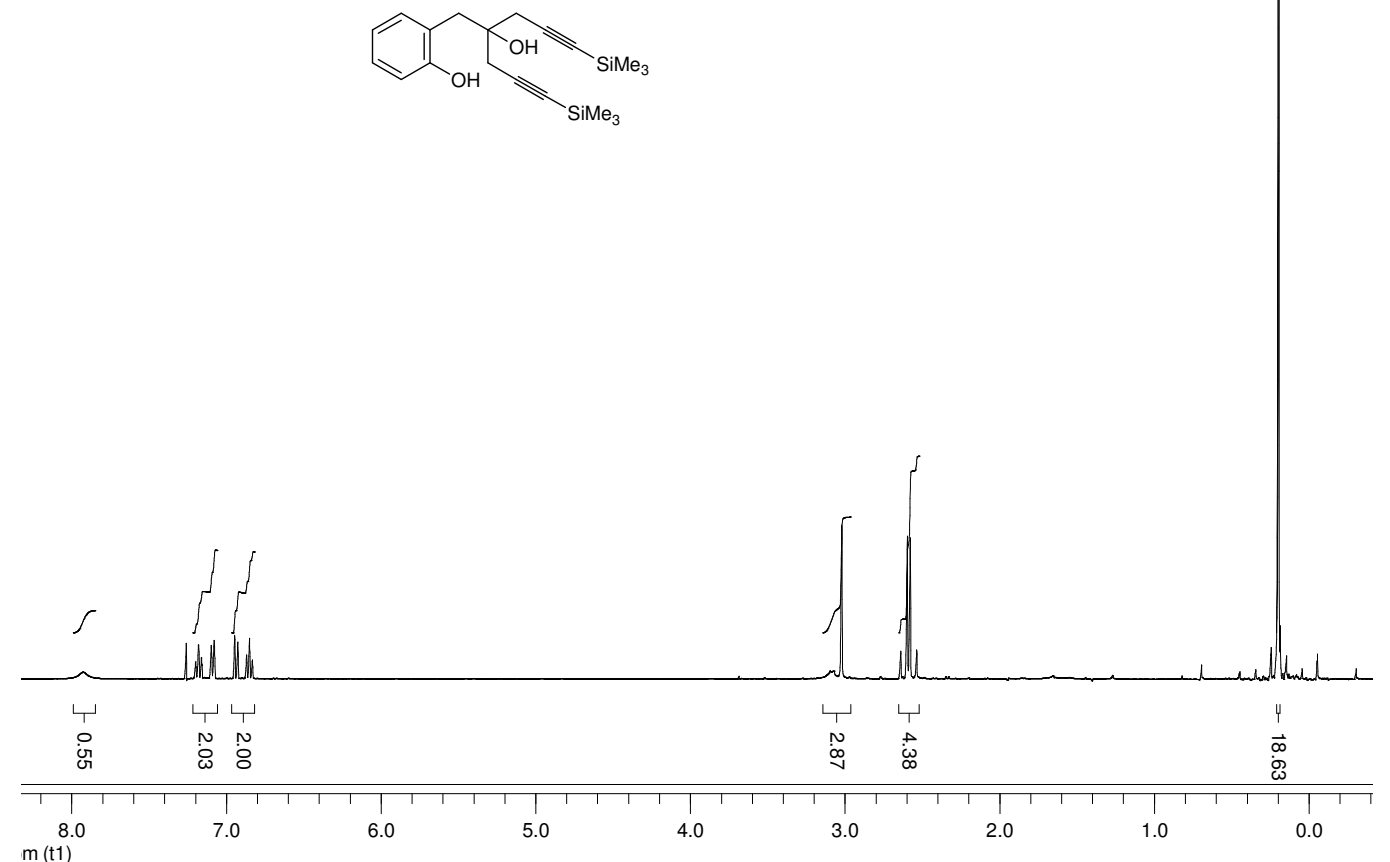

SI6 $\left({ }^{13} \mathrm{C}\right)$ :
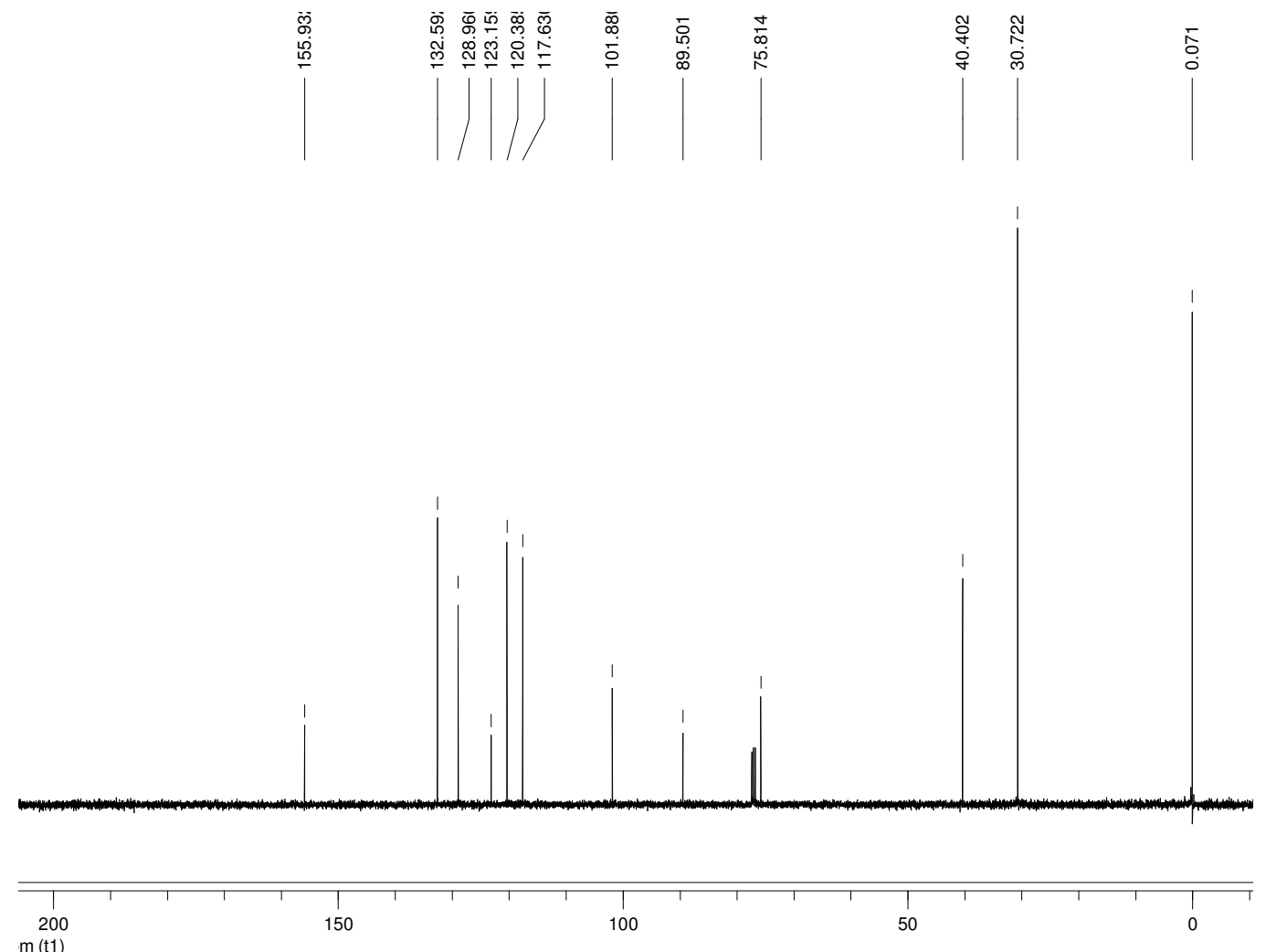
SI7 $\left({ }^{1} H\right):$
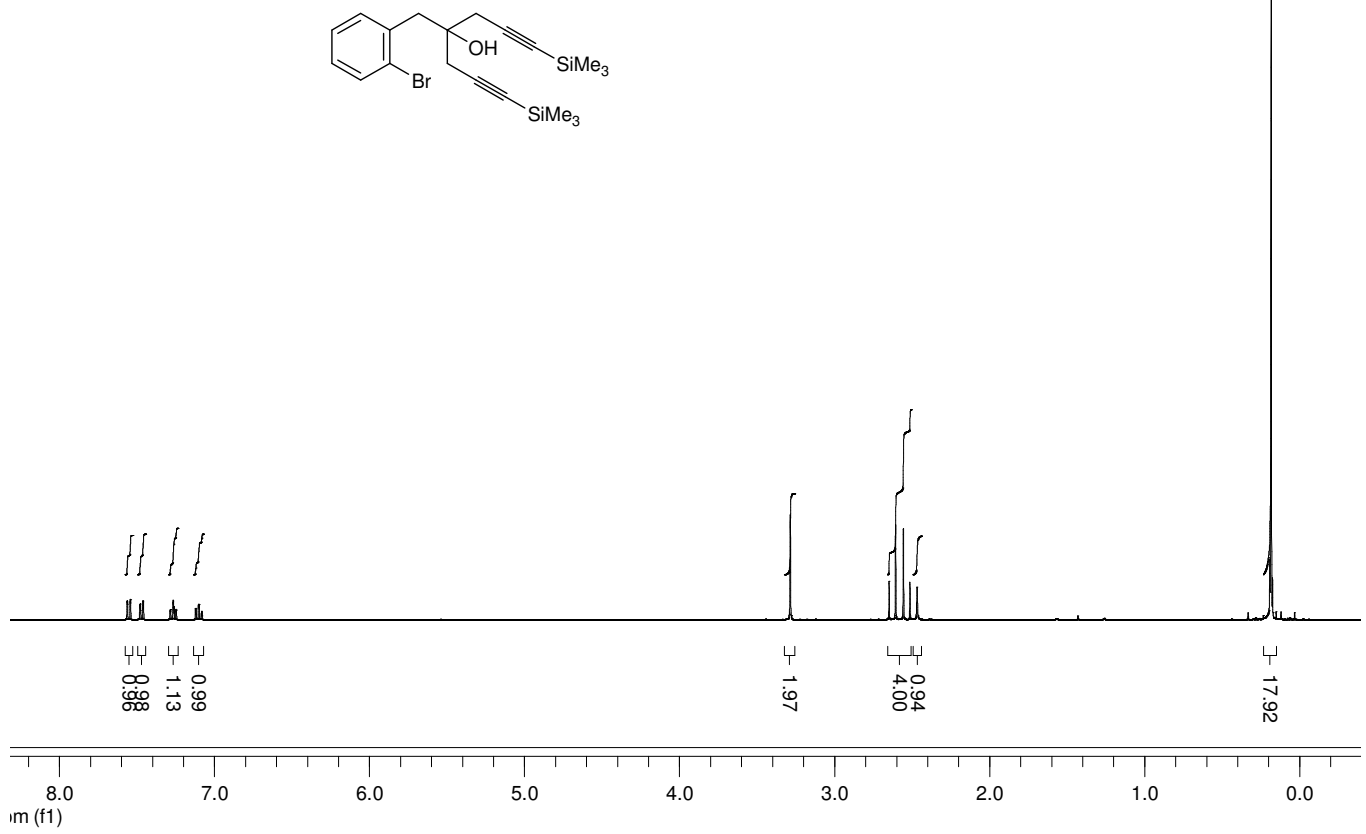

$\operatorname{SI7}\left({ }^{13} \mathrm{C}\right)$ :

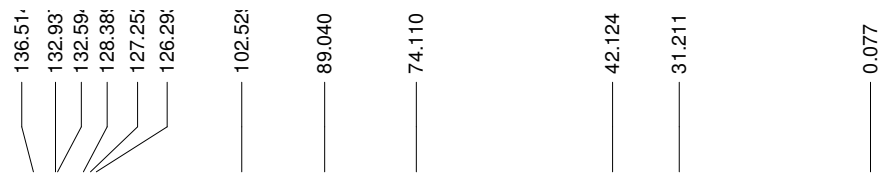

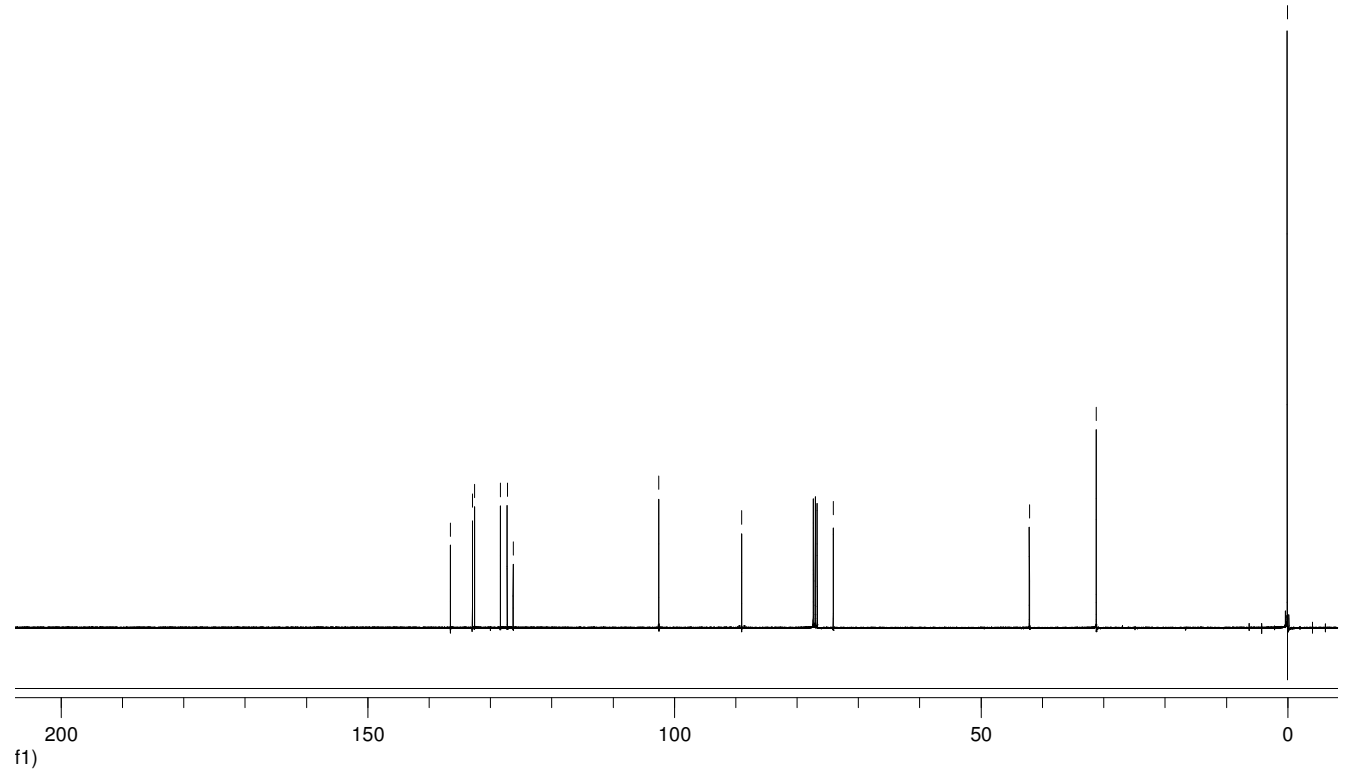


SI8 $\left({ }^{1} H\right)$ :

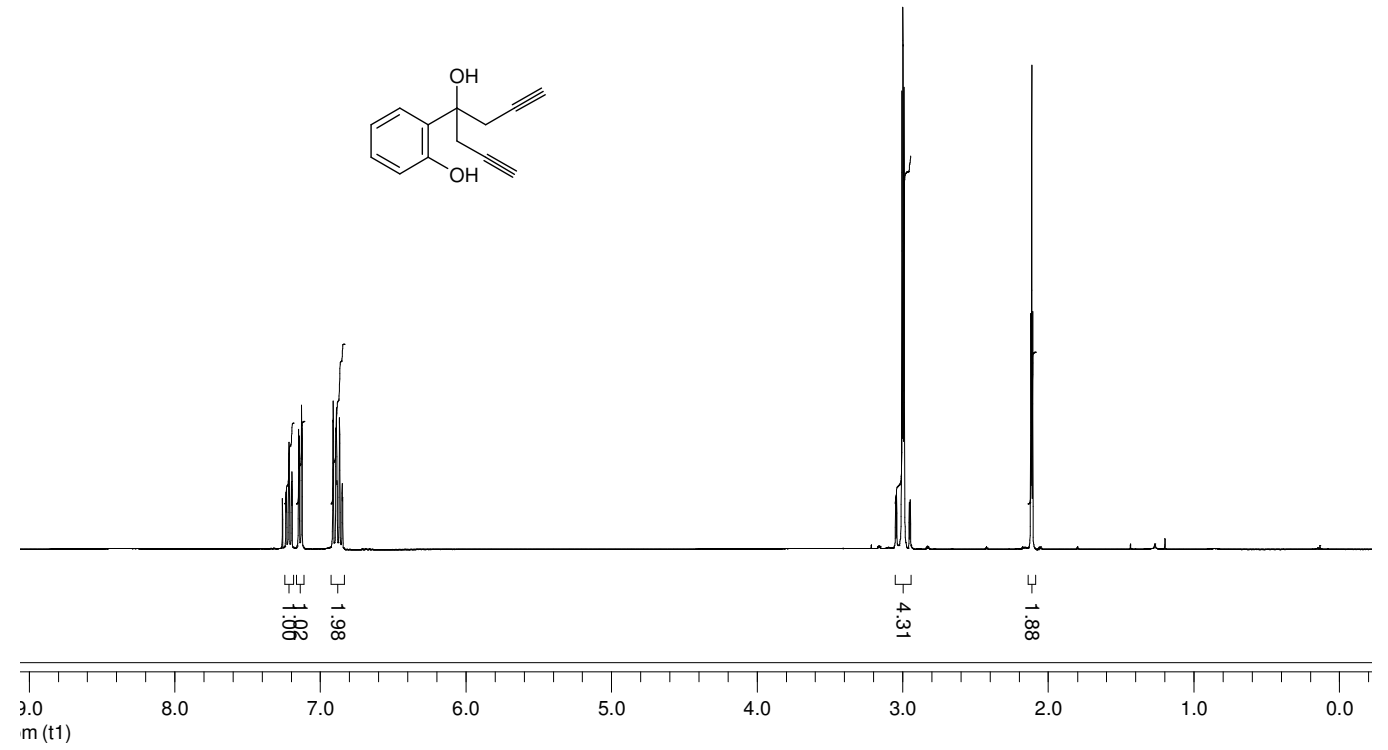

SI8 $\left({ }^{13} \mathrm{C}\right):$

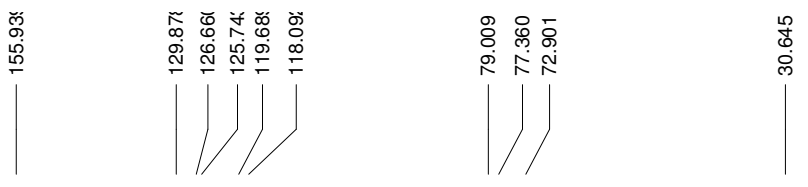
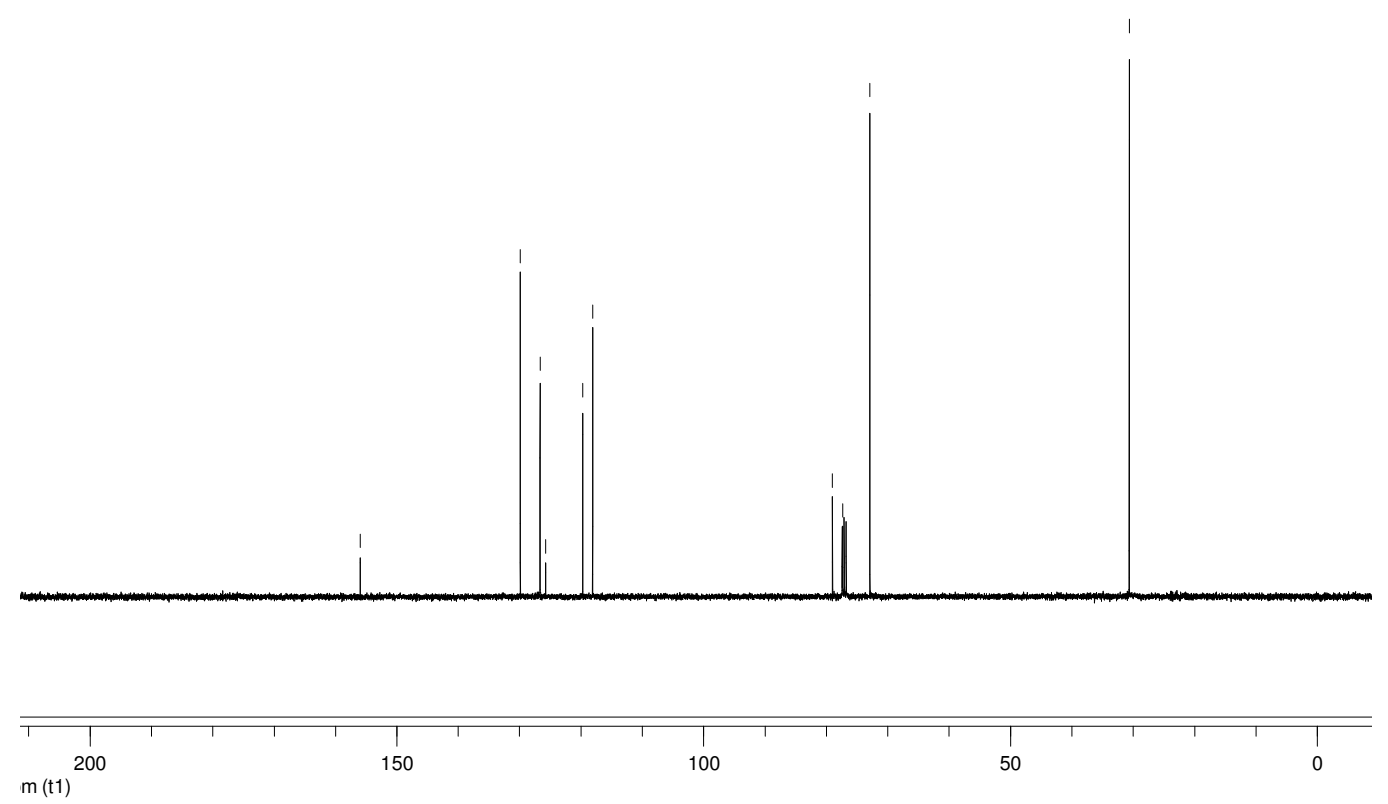
SI9 $\left({ }^{1} H\right)$ :

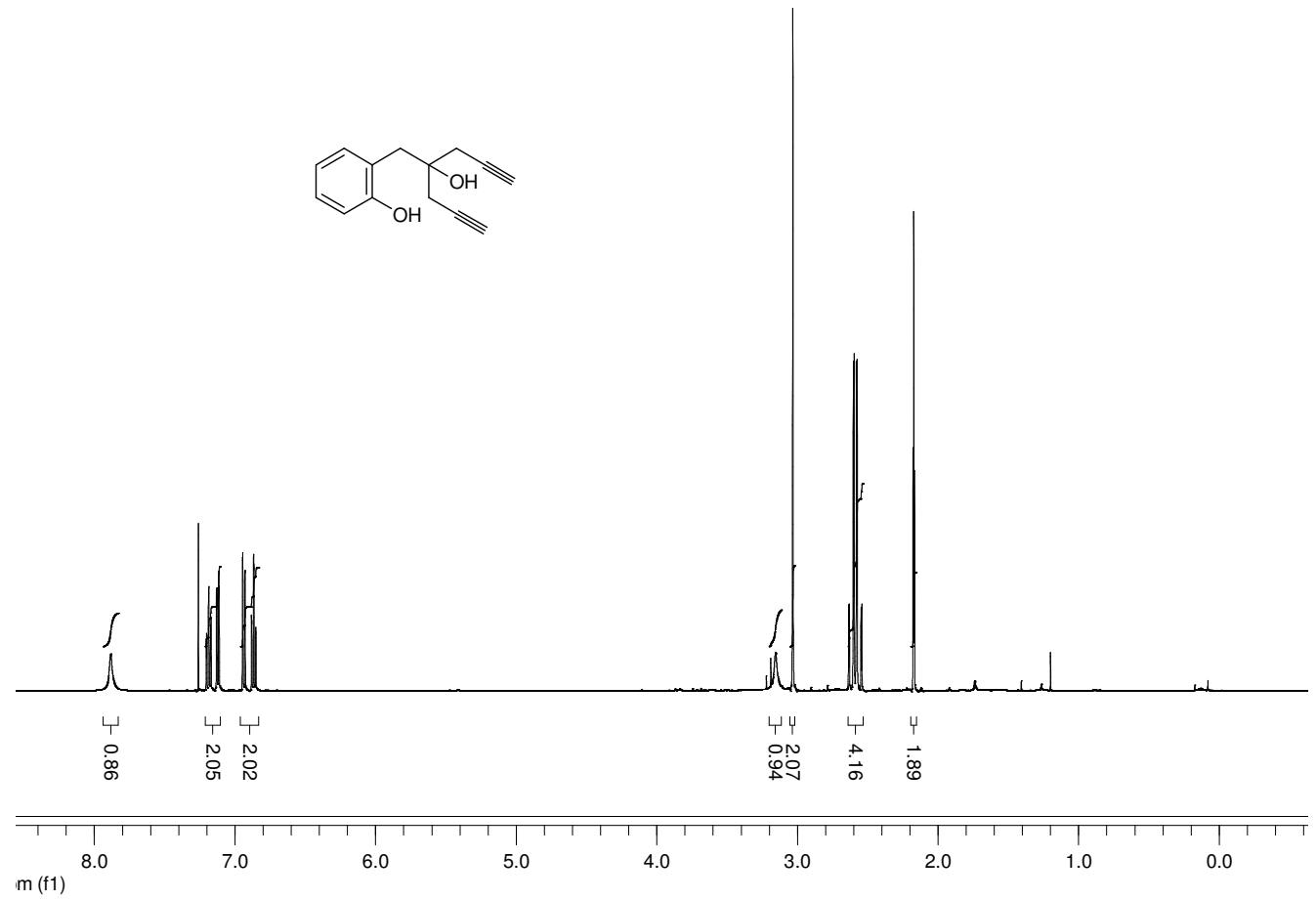

SI9 $\left({ }^{13} \mathrm{C}\right)$ :

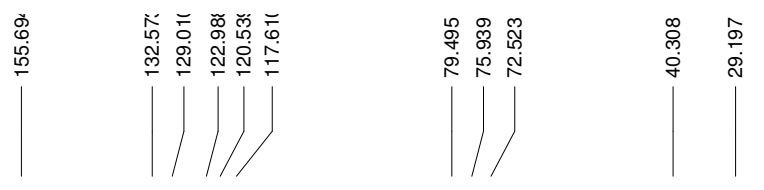
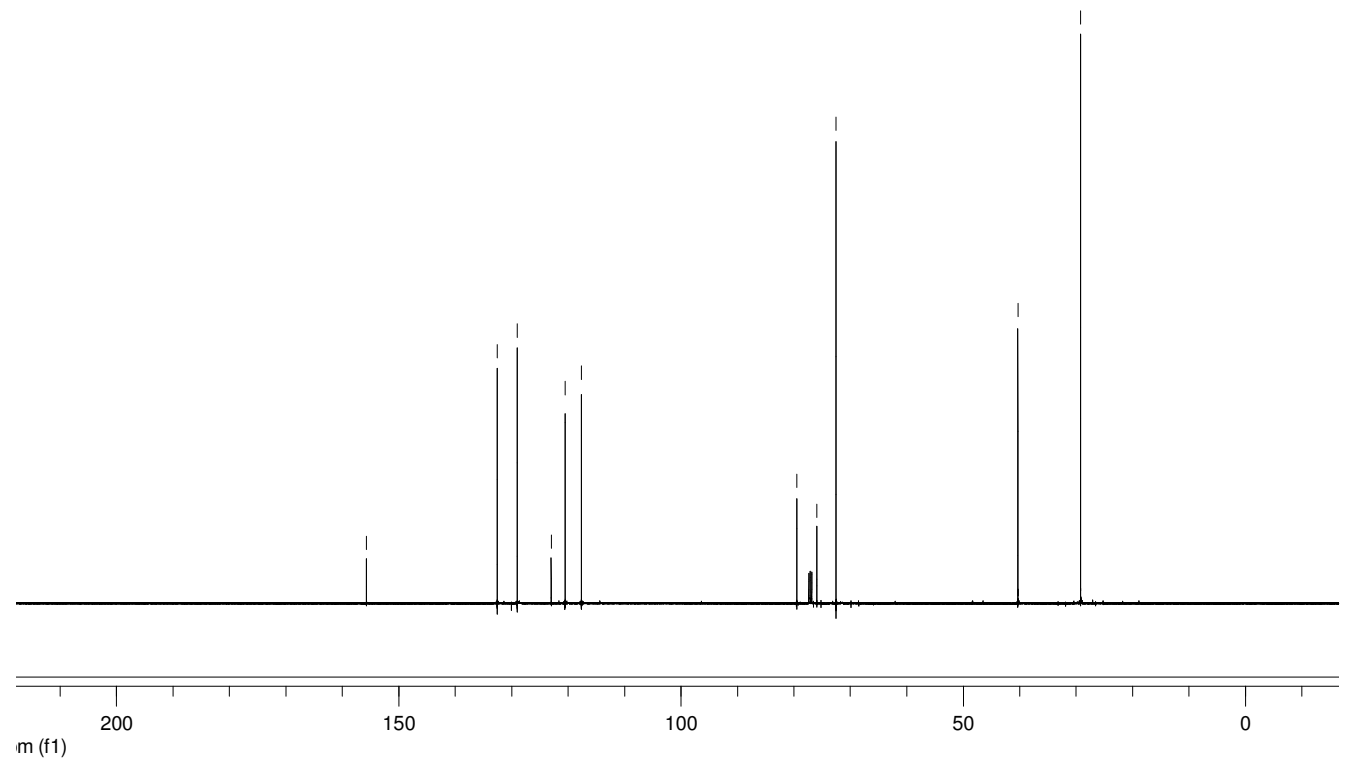


\section{SI10 $\left({ }^{1} \mathrm{H}\right):$}

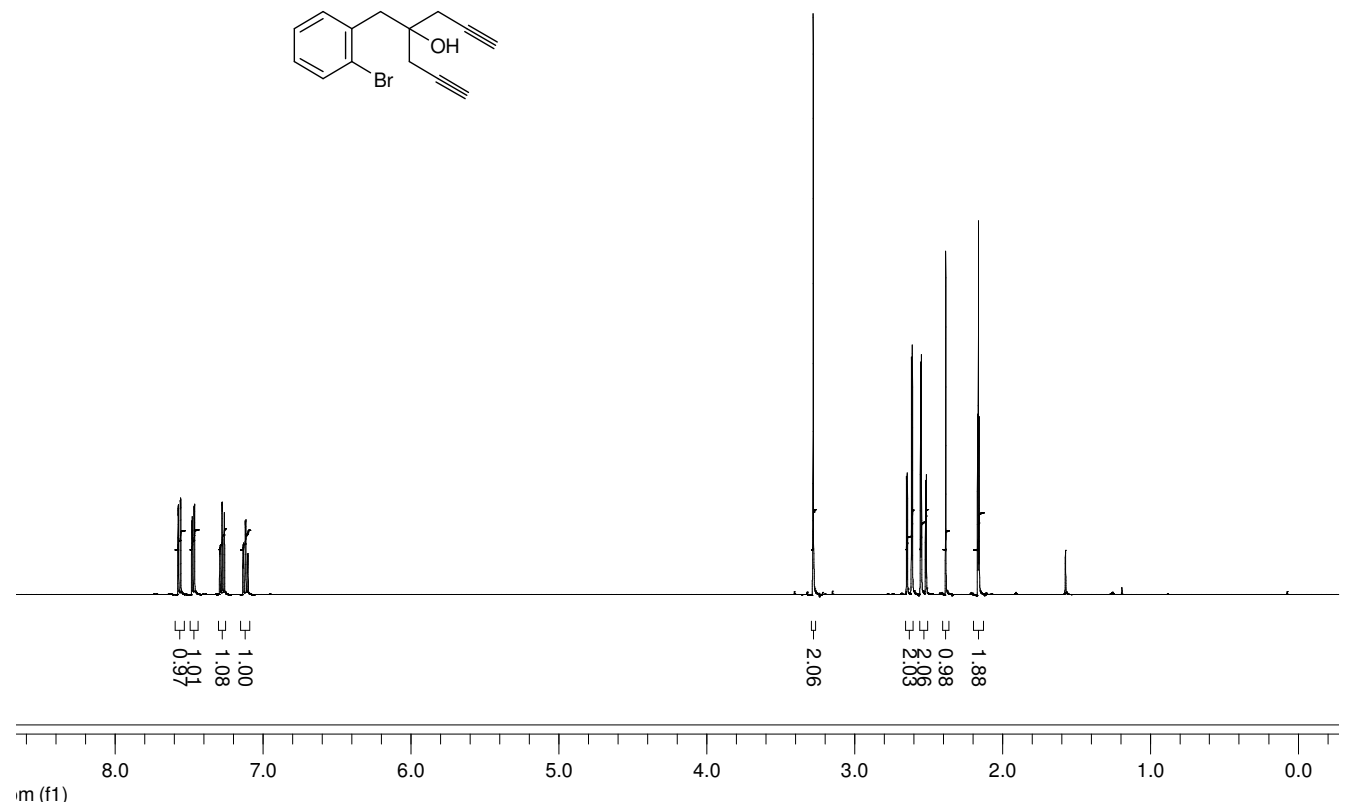

SI10 $\left({ }^{13} \mathrm{C}\right)$ :

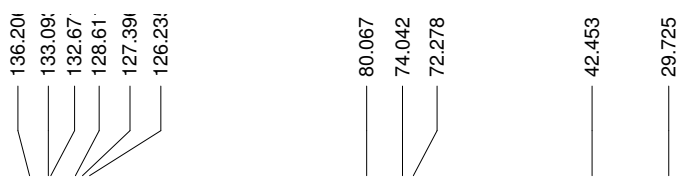

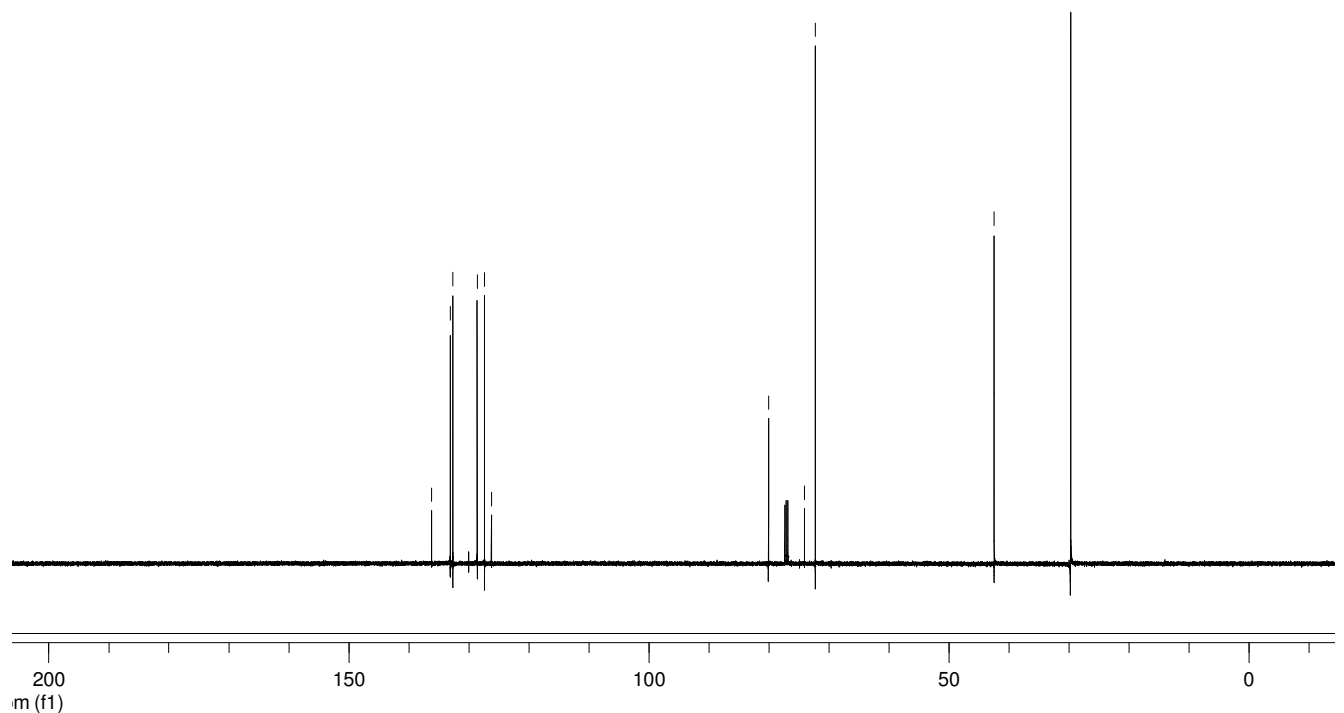




\section{Sl11 $\left({ }^{1} H\right)$ :}

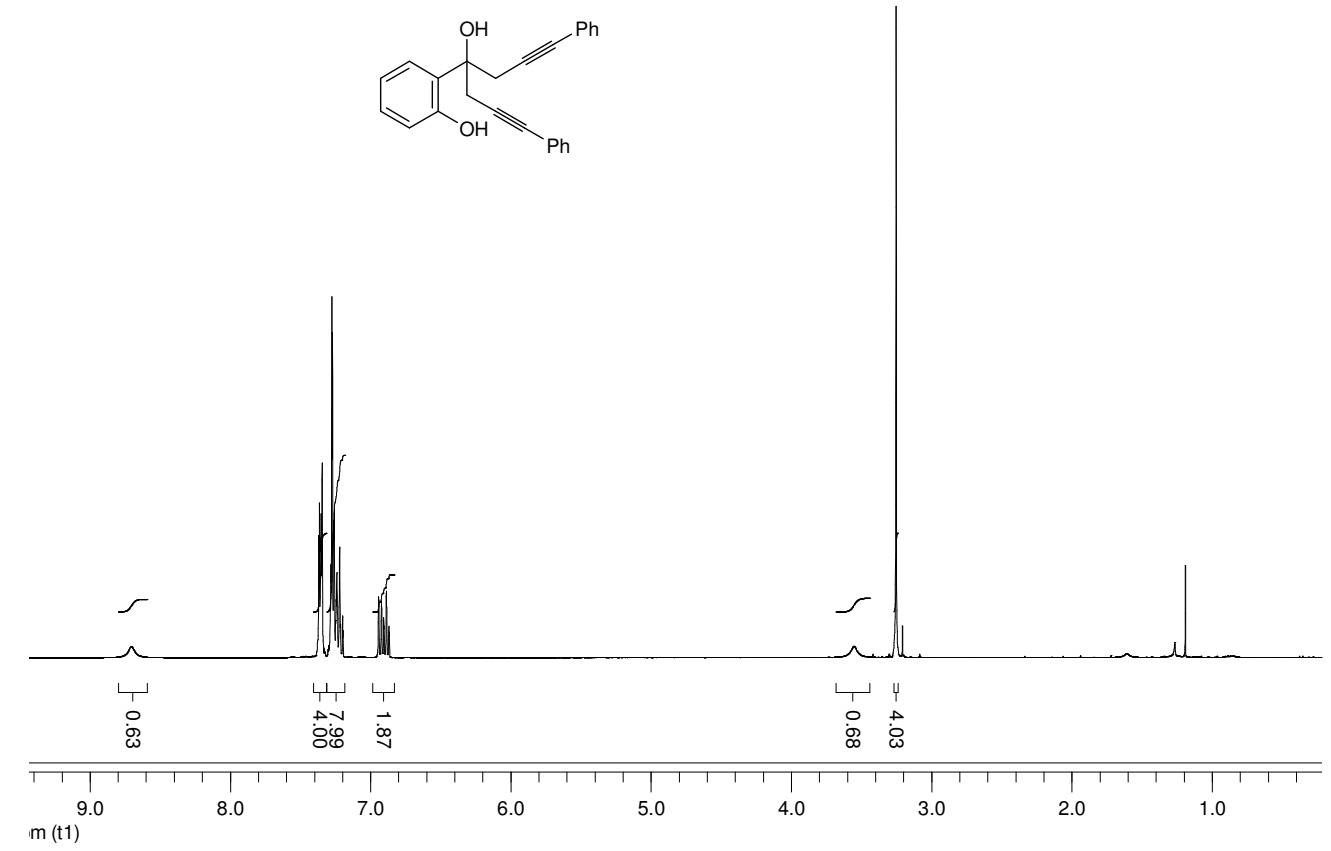

\section{SI11 $\left({ }^{13} \mathrm{C}\right)$ :}
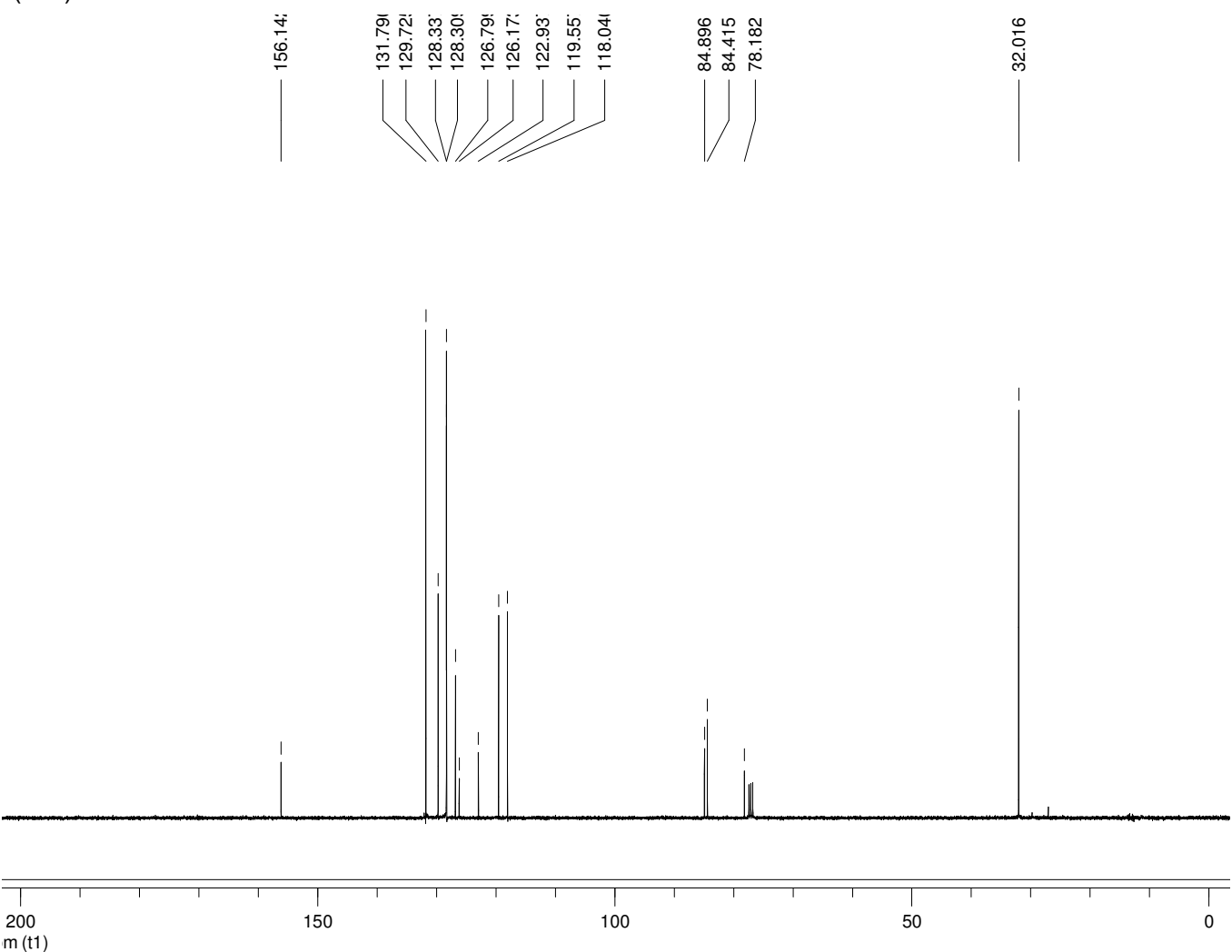


\section{SI12 $\left({ }^{1} \mathrm{H}\right)$ :}

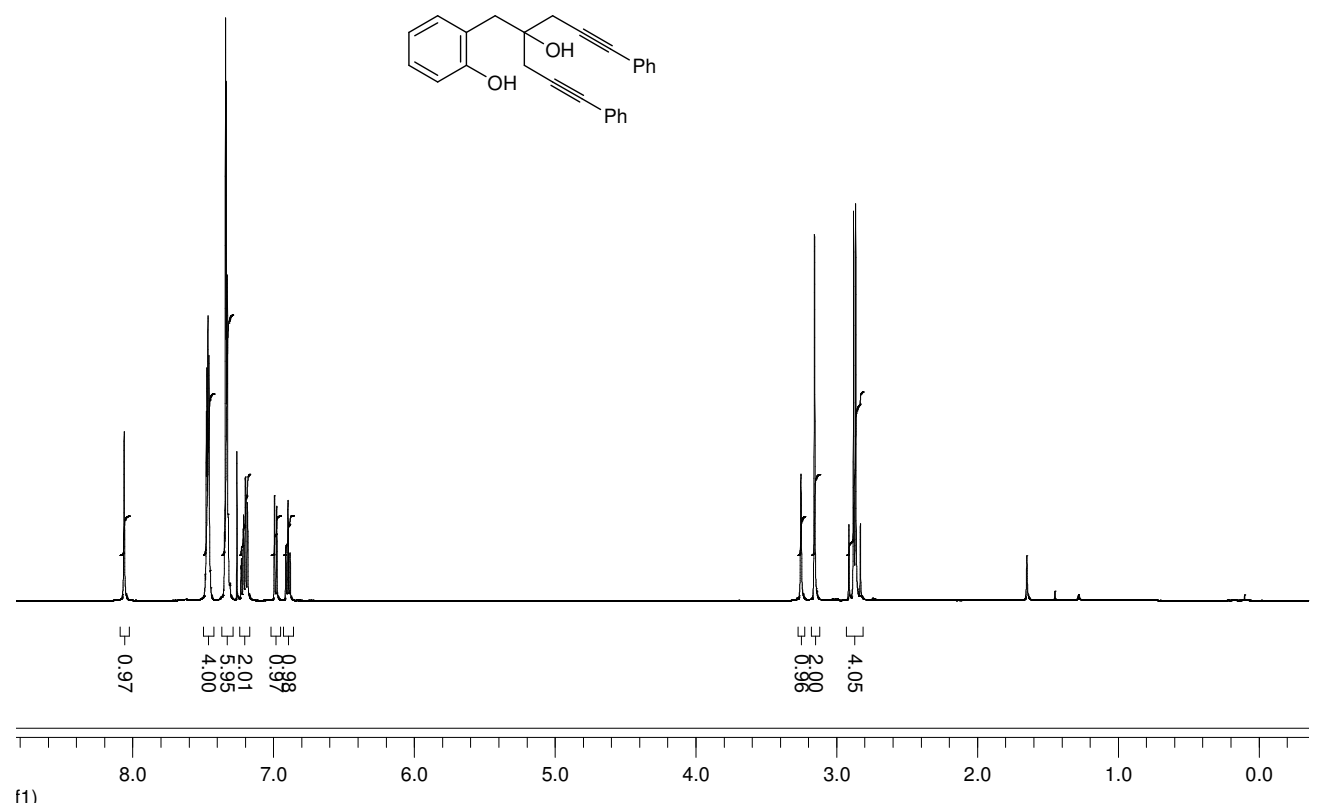

f1)

SI12 $\left({ }^{13} \mathrm{C}\right)$ :

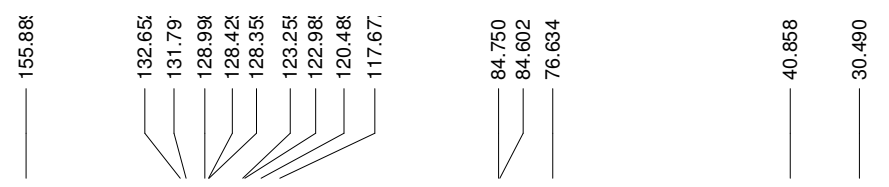

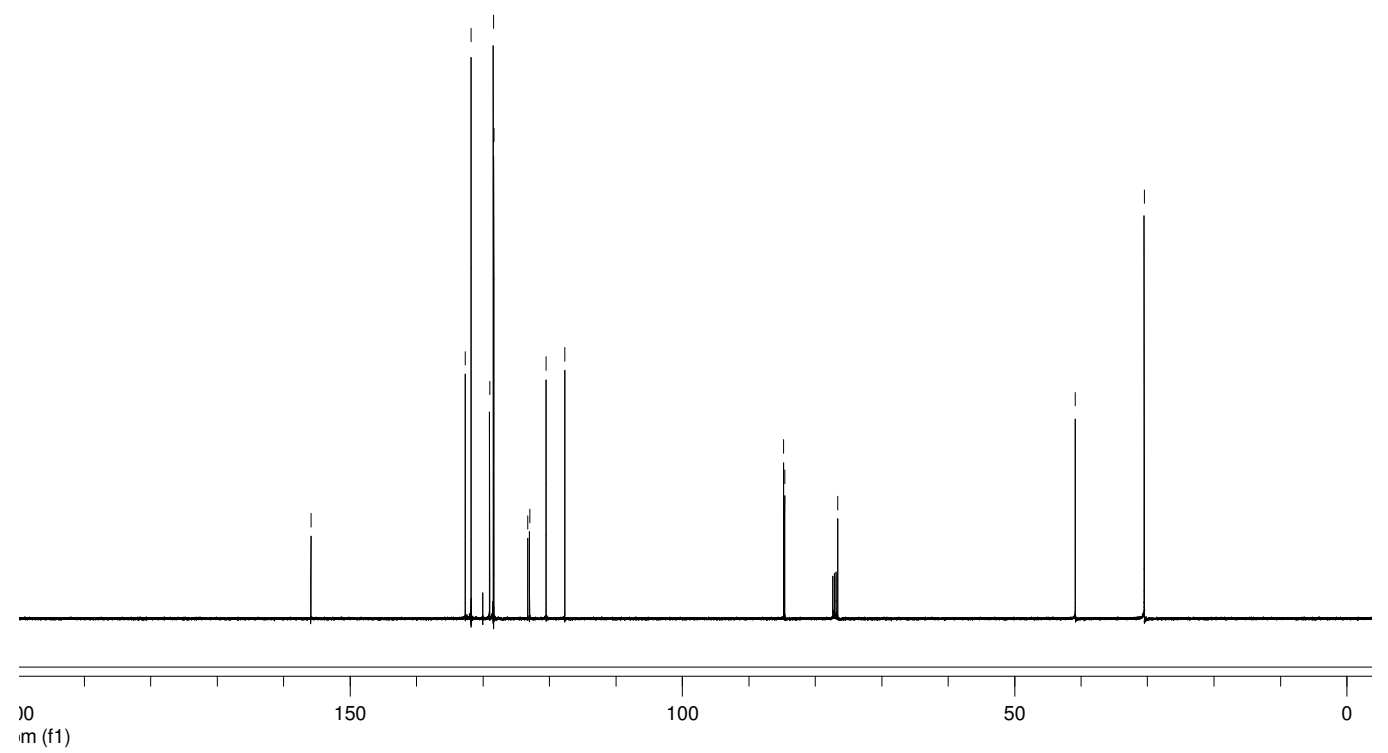


SI13 $\left({ }^{1} H\right)$ :

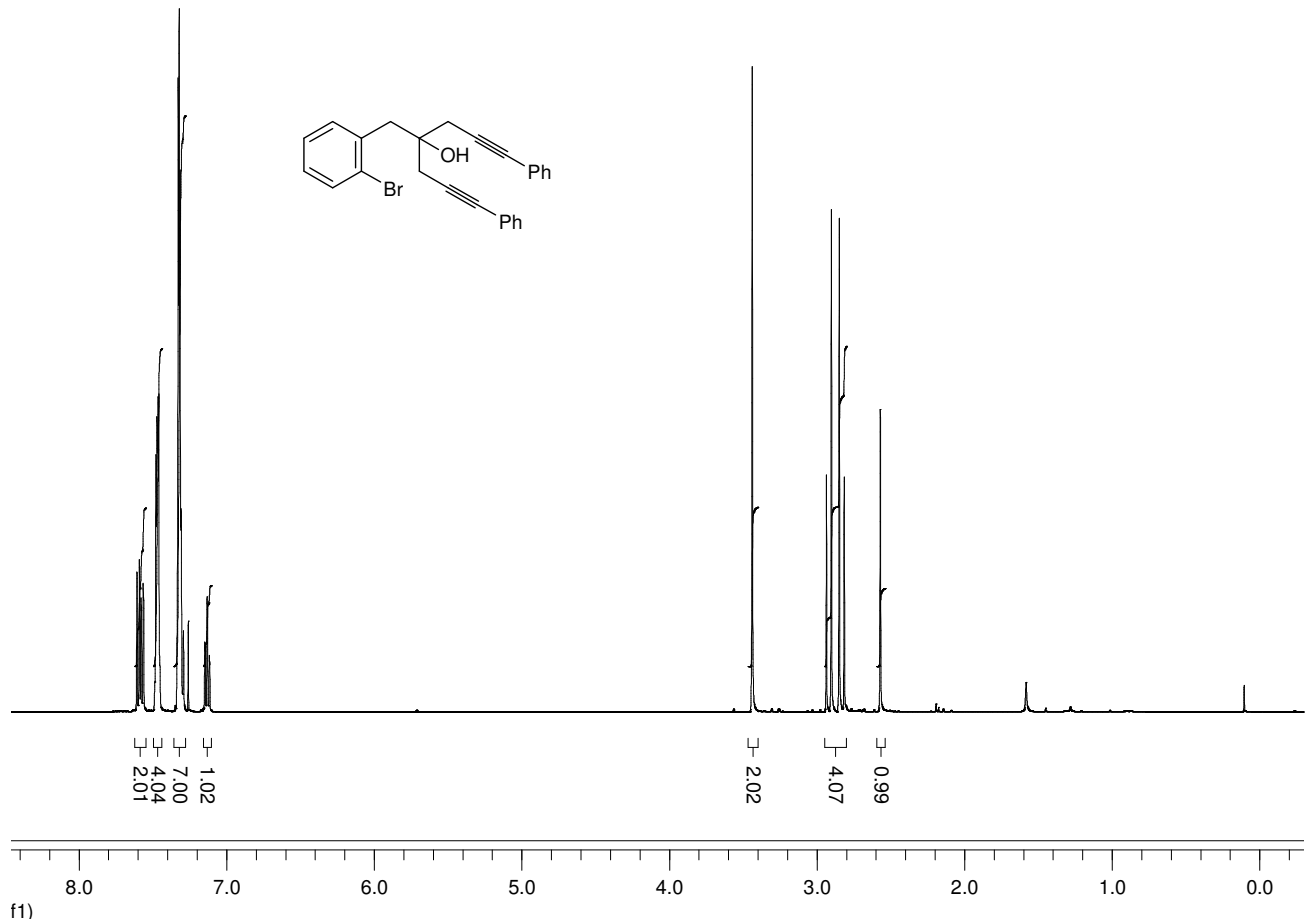

f1)

SI13 $\left({ }^{13} \mathrm{C}\right)$ :

ํํำ

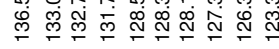

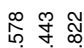

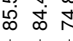

$\lfloor|\downarrow|\rfloor\rfloor$
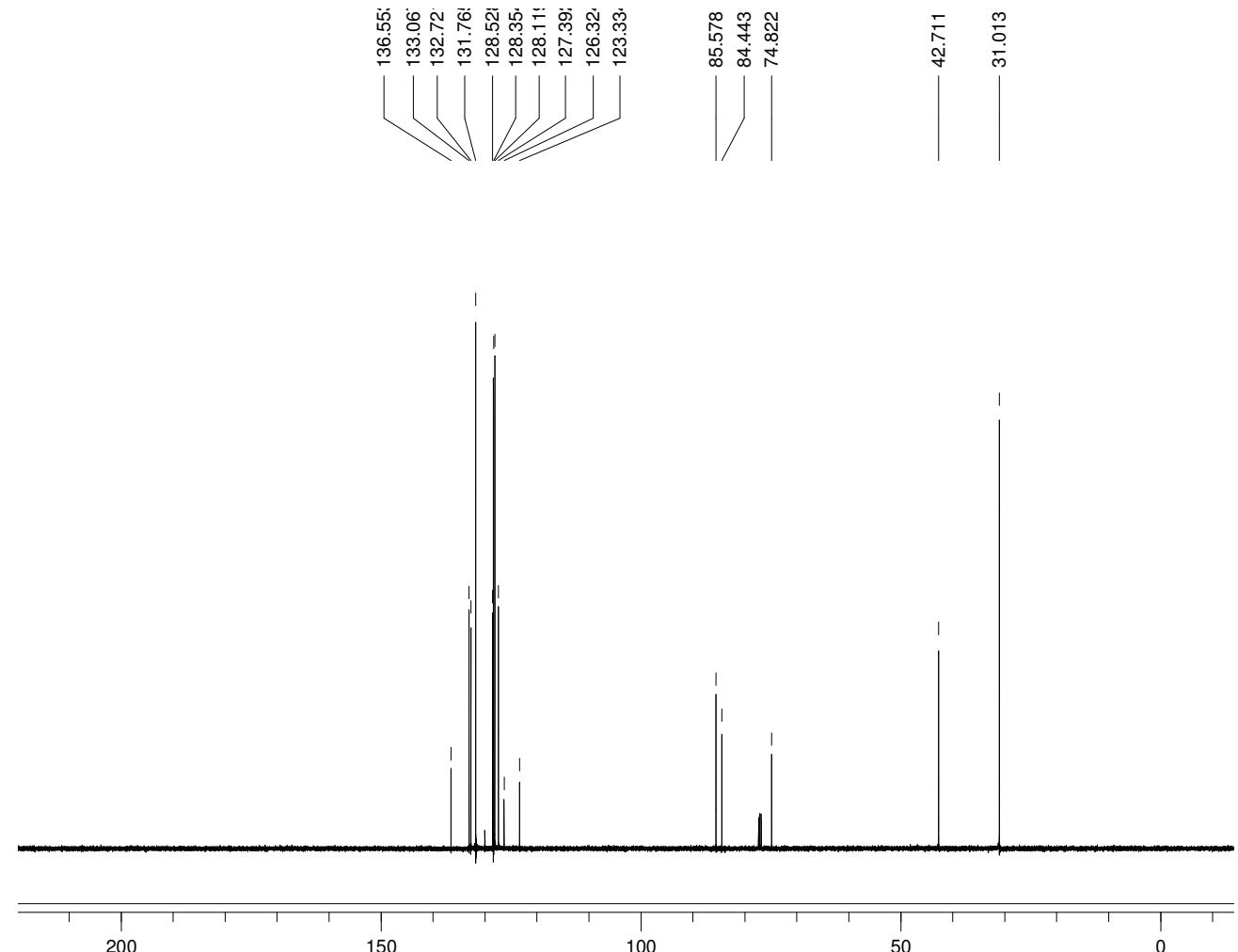

150

100

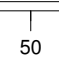

f1) 


\section{(E,E)-SI14 $\left({ }^{1} \mathrm{H}\right)$ :}

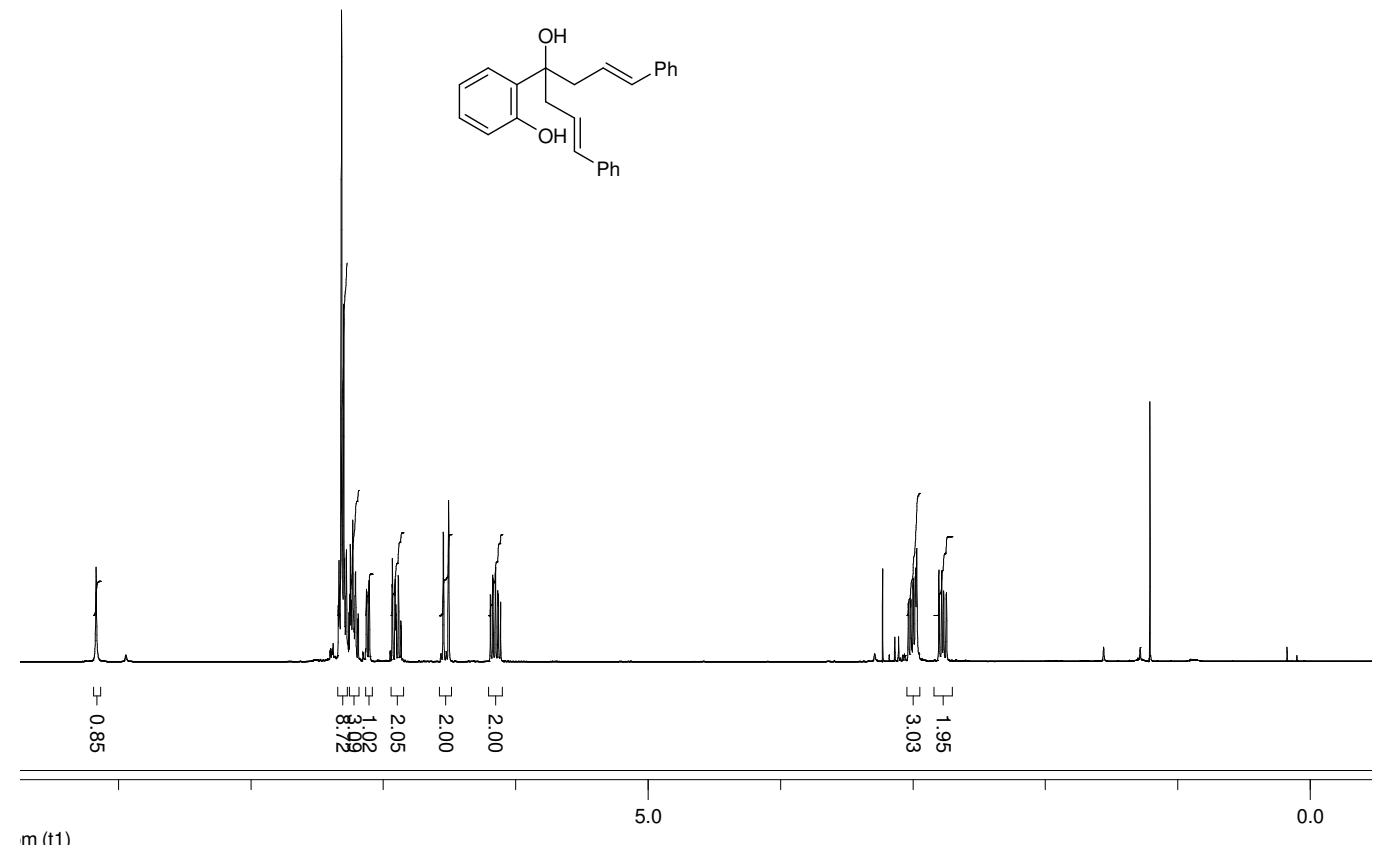

(E,E)-SI14 $\left({ }^{13} \mathrm{C}\right)$ :
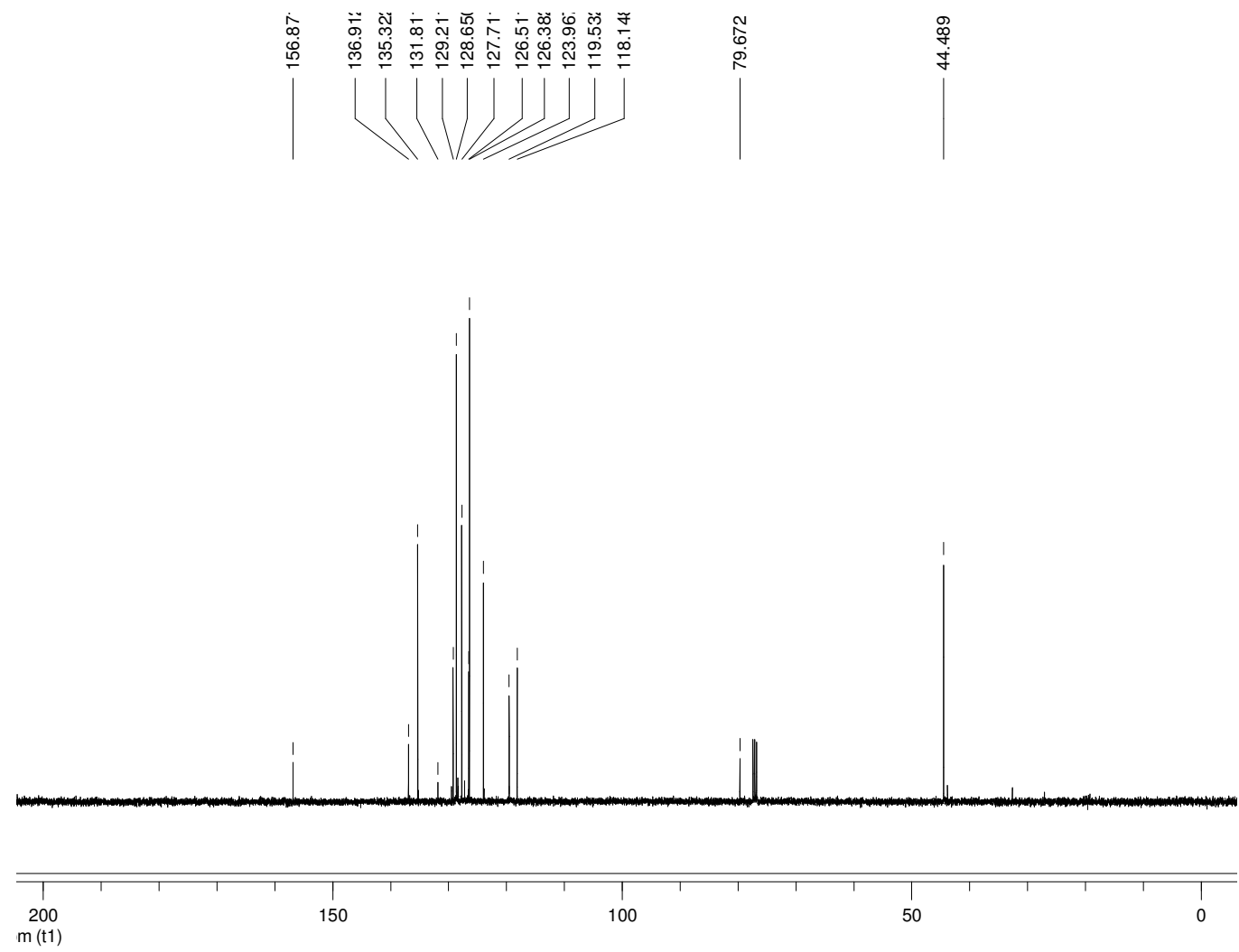


\section{$(E, E)-\mathbf{S I} 15\left({ }^{1} H\right)$ :}
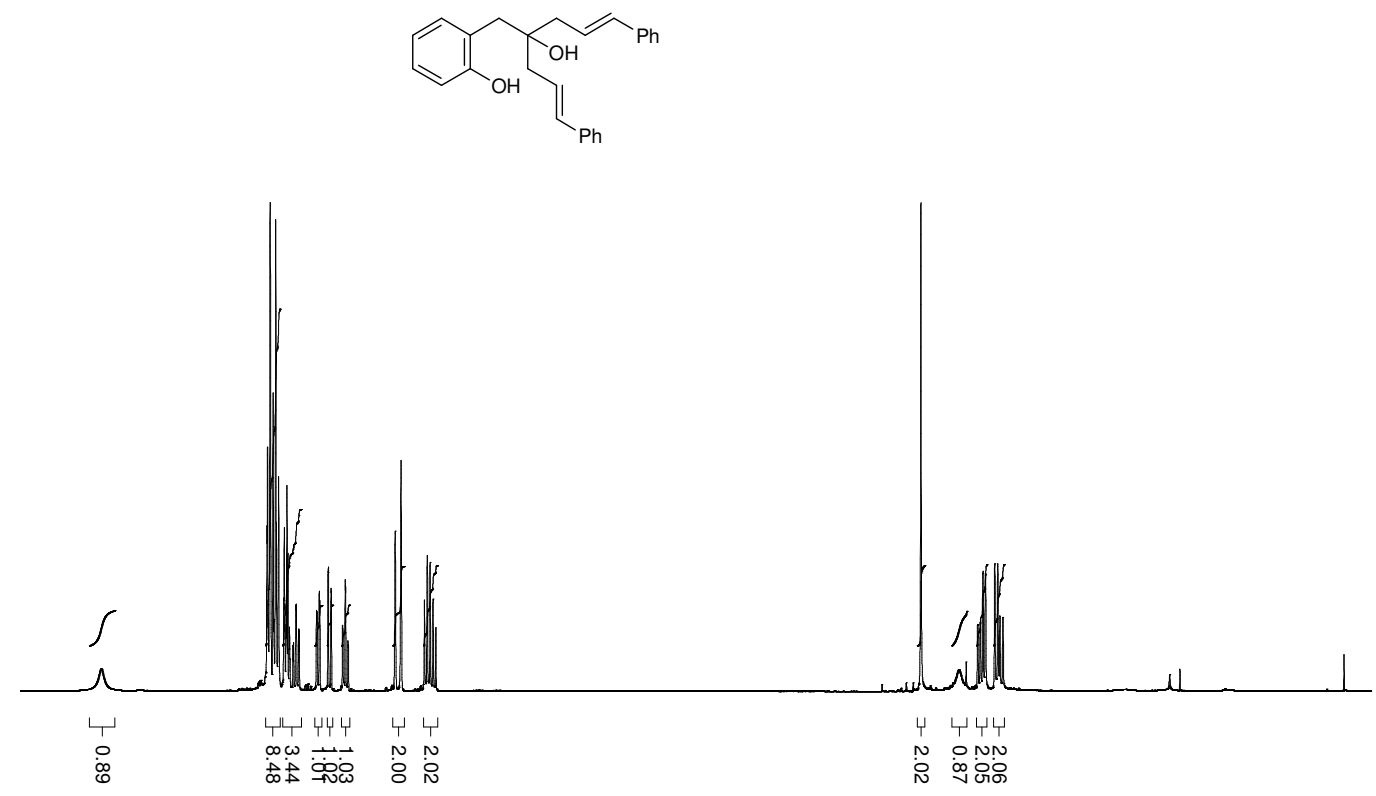

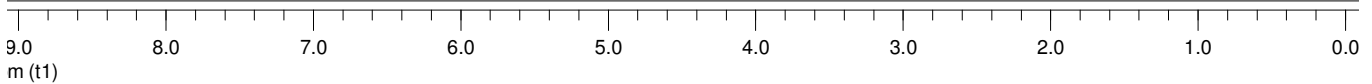

$(E, E)-\mathbf{S I 1 5}\left({ }^{13} \mathrm{C}\right)$ :
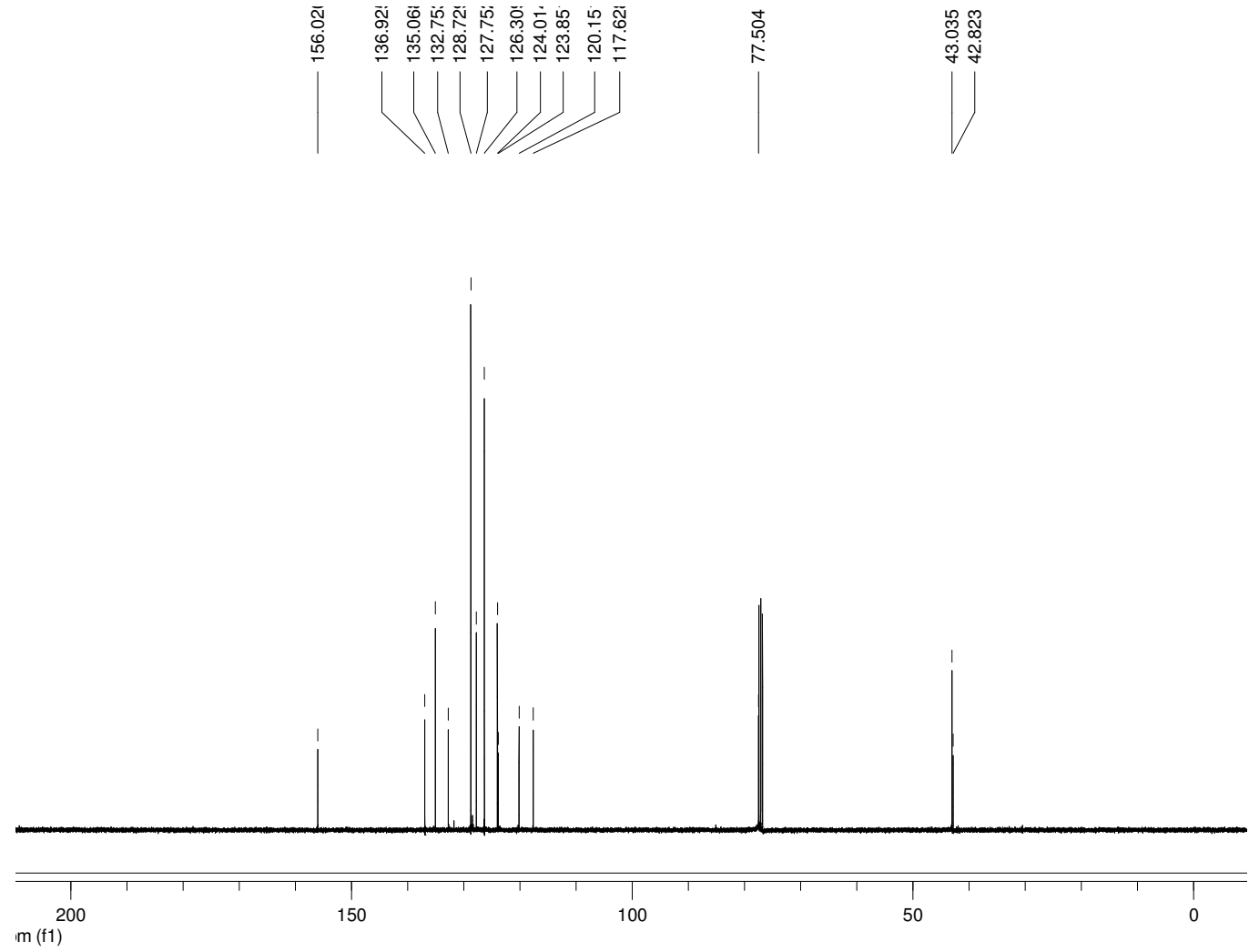


\section{$(E, E)-\operatorname{SI17}\left({ }^{1} H\right)$ :}
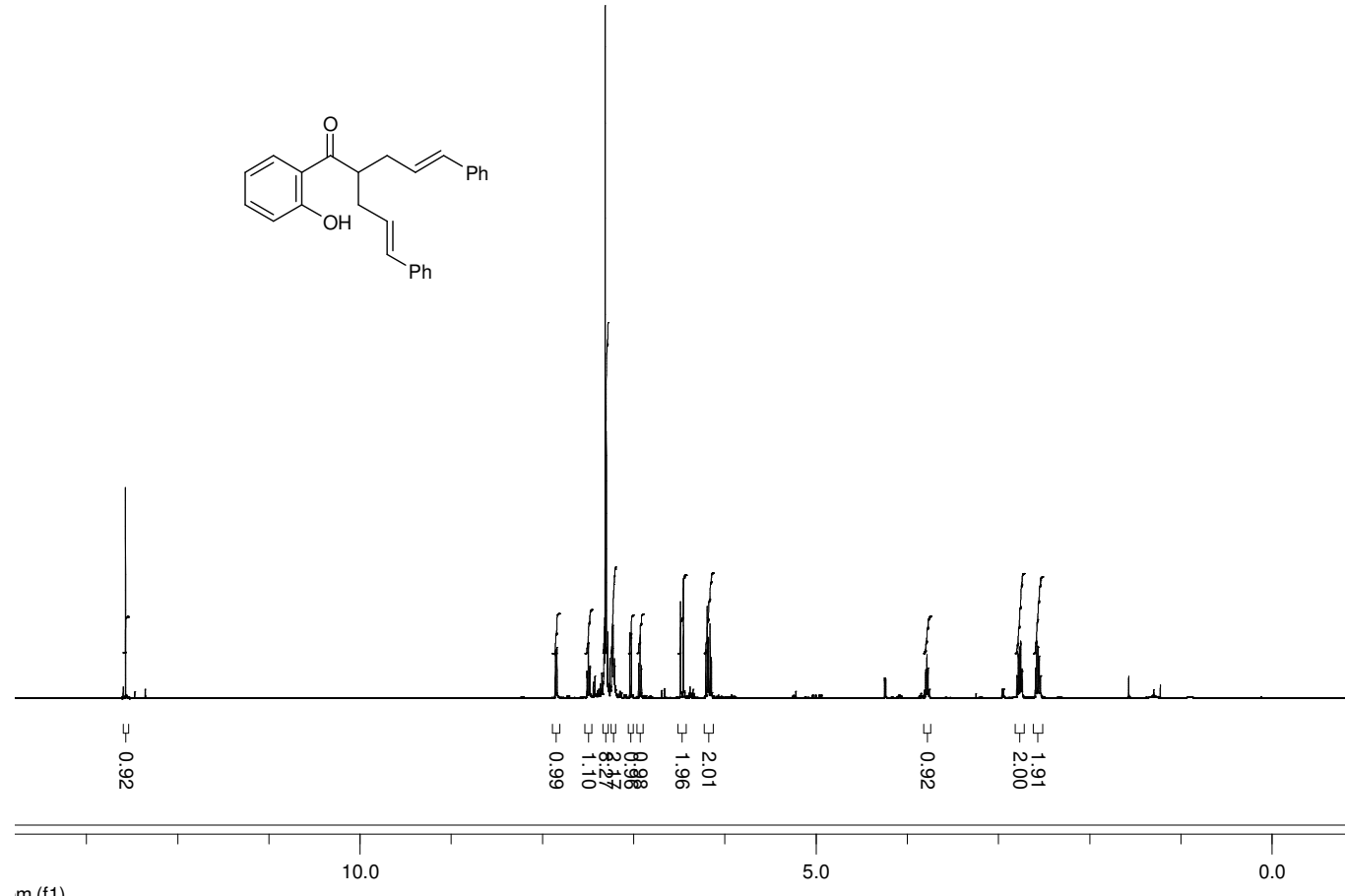

m (f1)

(E,E)-SI17 $\left({ }^{13} \mathrm{C}\right)$ :

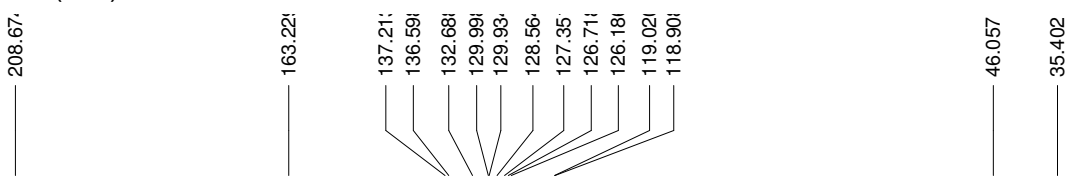
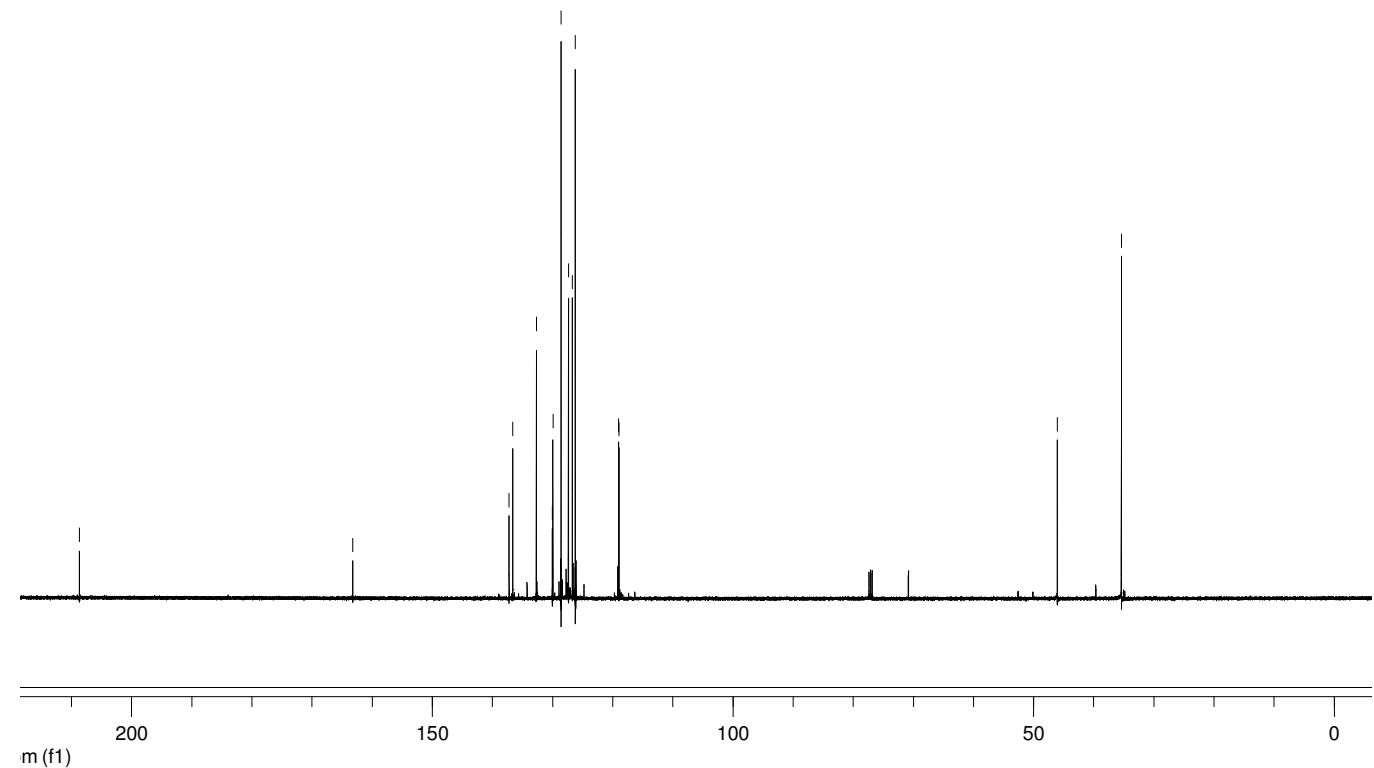


\section{$(E, E)-\mathbf{S I} 18\left({ }^{1} \mathrm{H}\right)$ :}

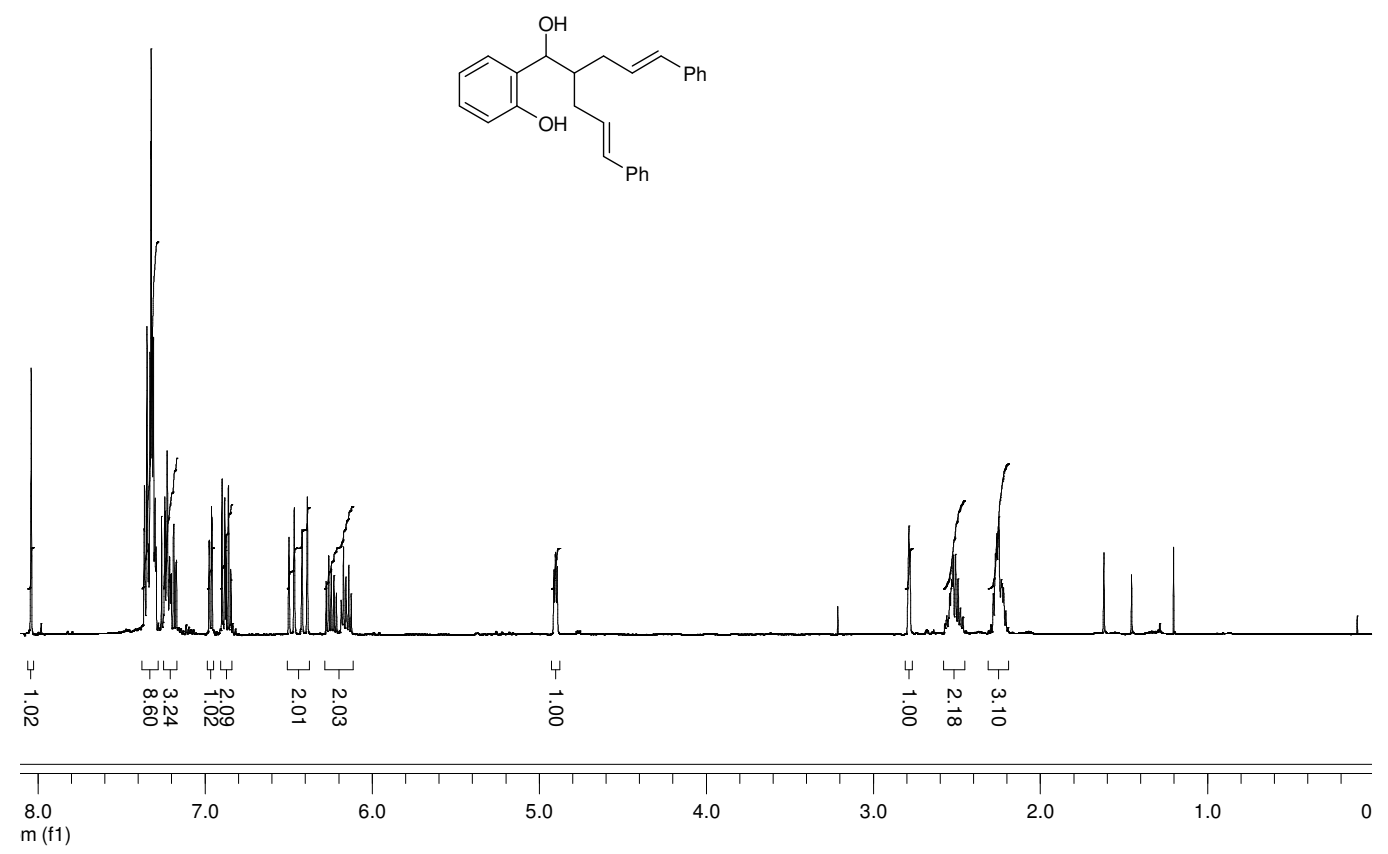

$(E, E)-\mathbf{S I 1 8}\left({ }^{13} \mathrm{C}\right)$ :

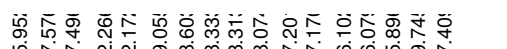

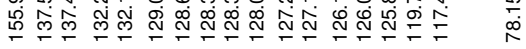

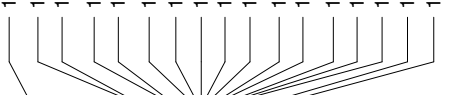

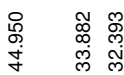
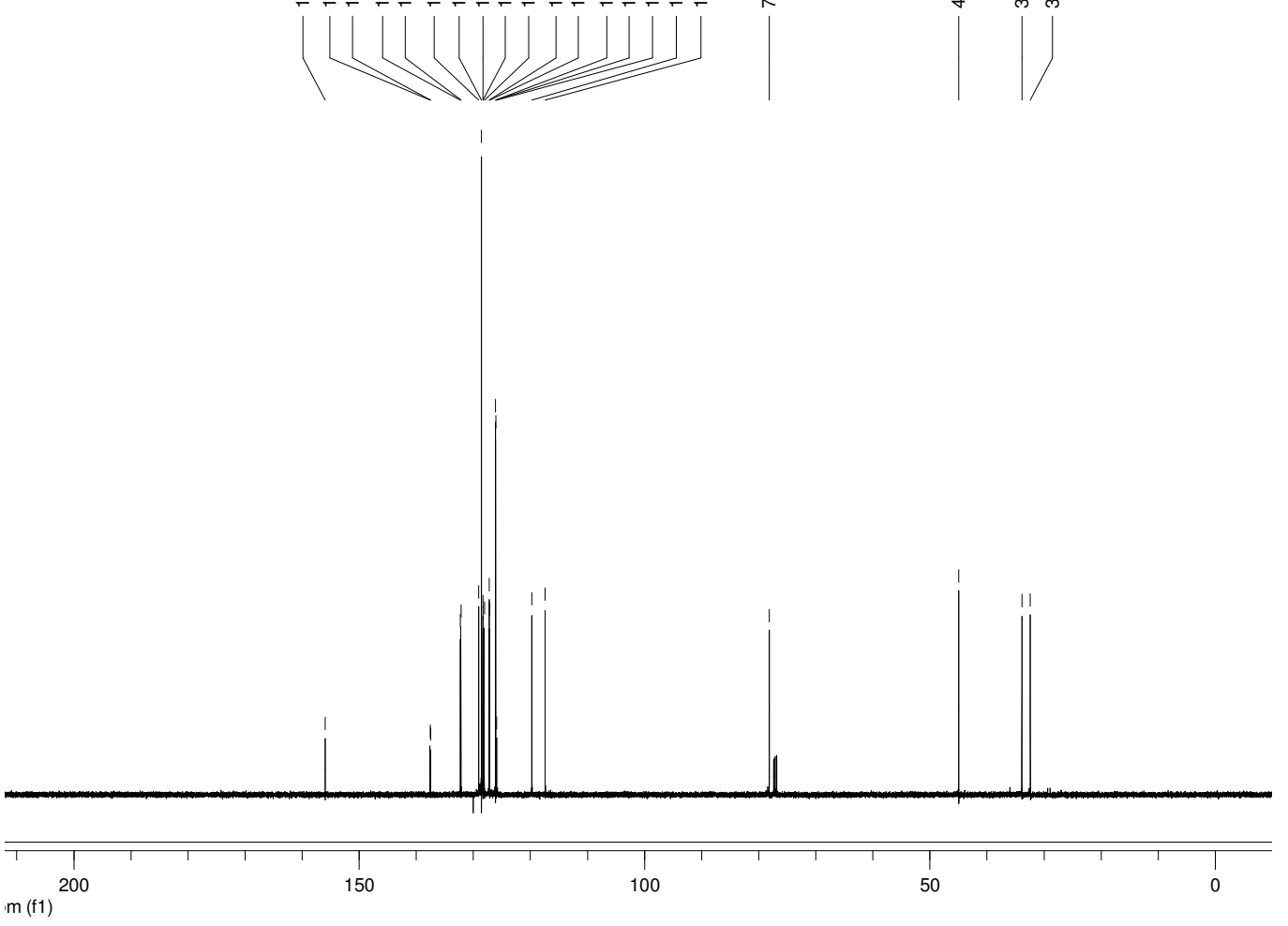


\section{$(E, E)-$ SI19 $\left({ }^{1} H\right)$ :}
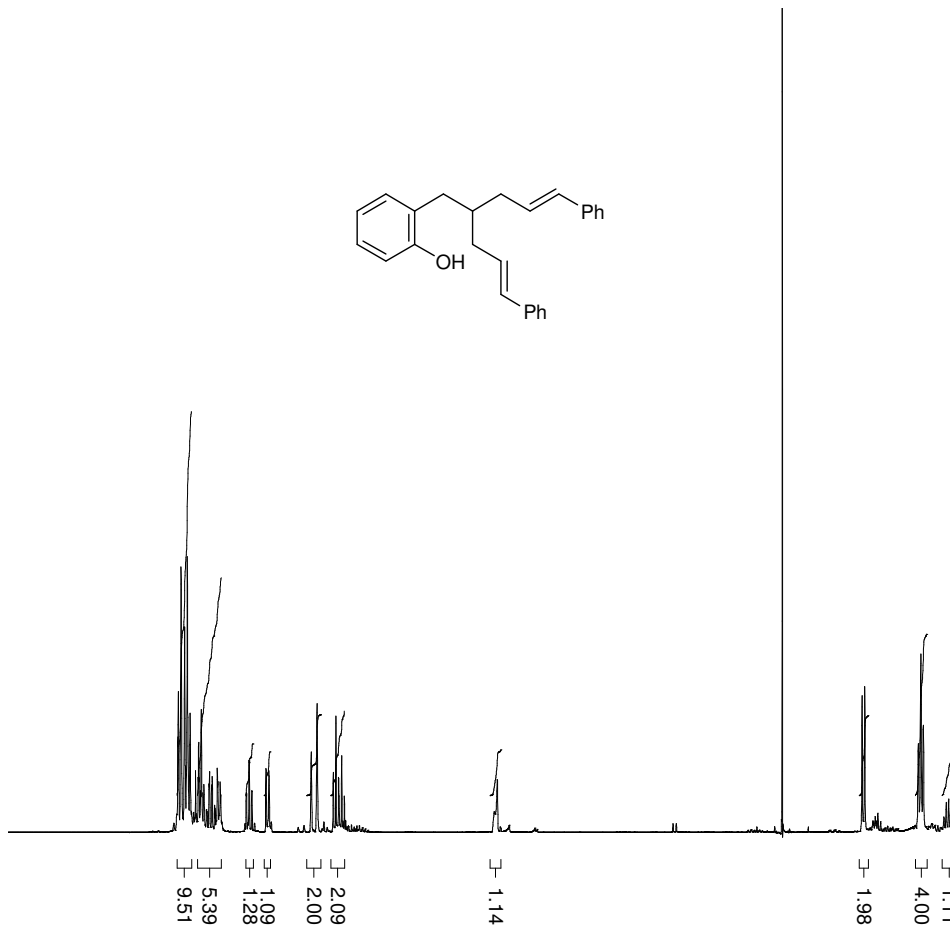

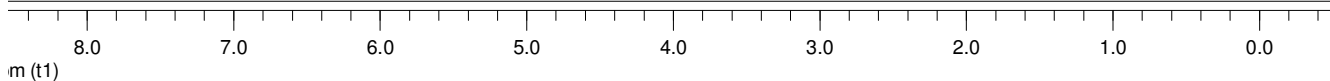

$(E, E)-\mathbf{S I 1 9}\left({ }^{13} \mathrm{C}\right)$ :

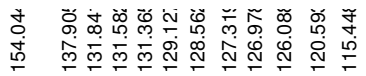

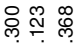

䆚

$1 /$

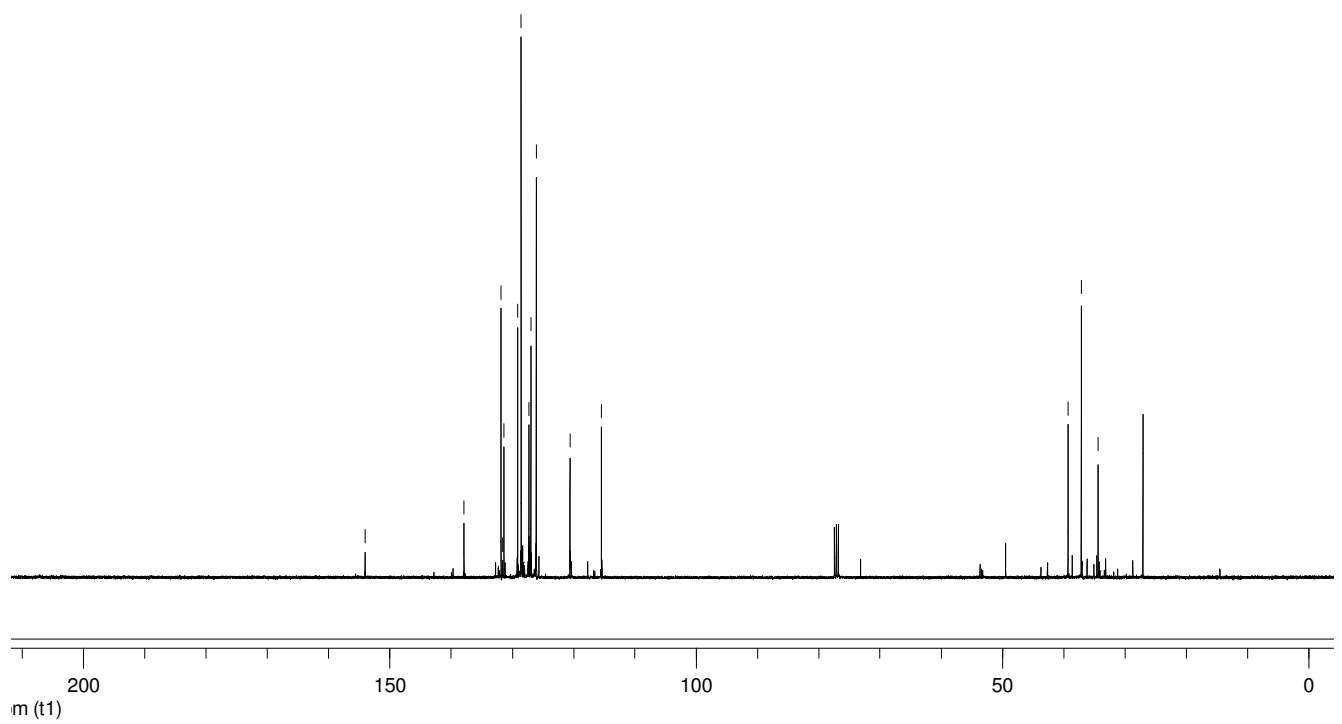




\section{SI20 $\left({ }^{1} \mathrm{H}\right):$}

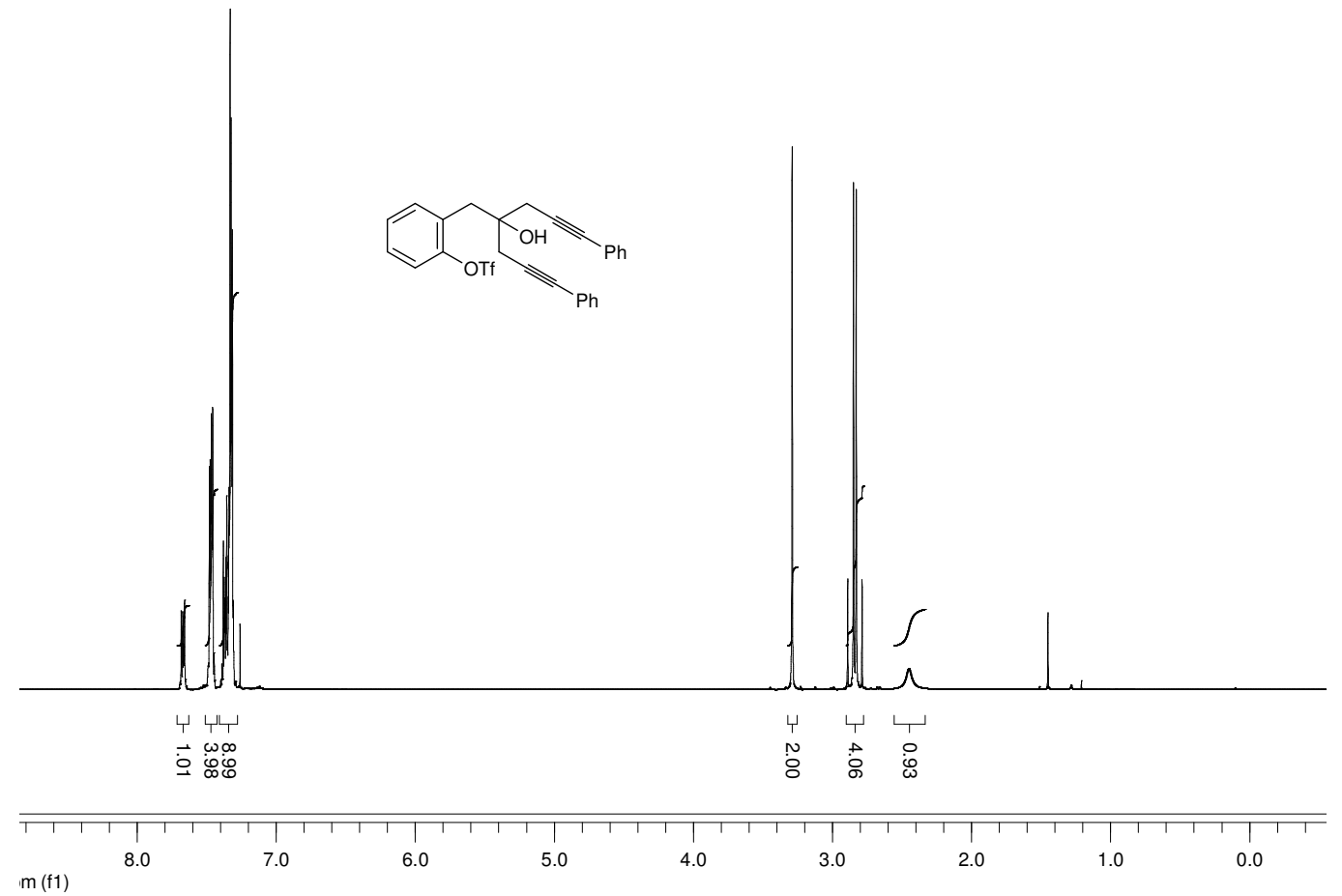

SI20 $\left({ }^{13} \mathrm{C}\right):$
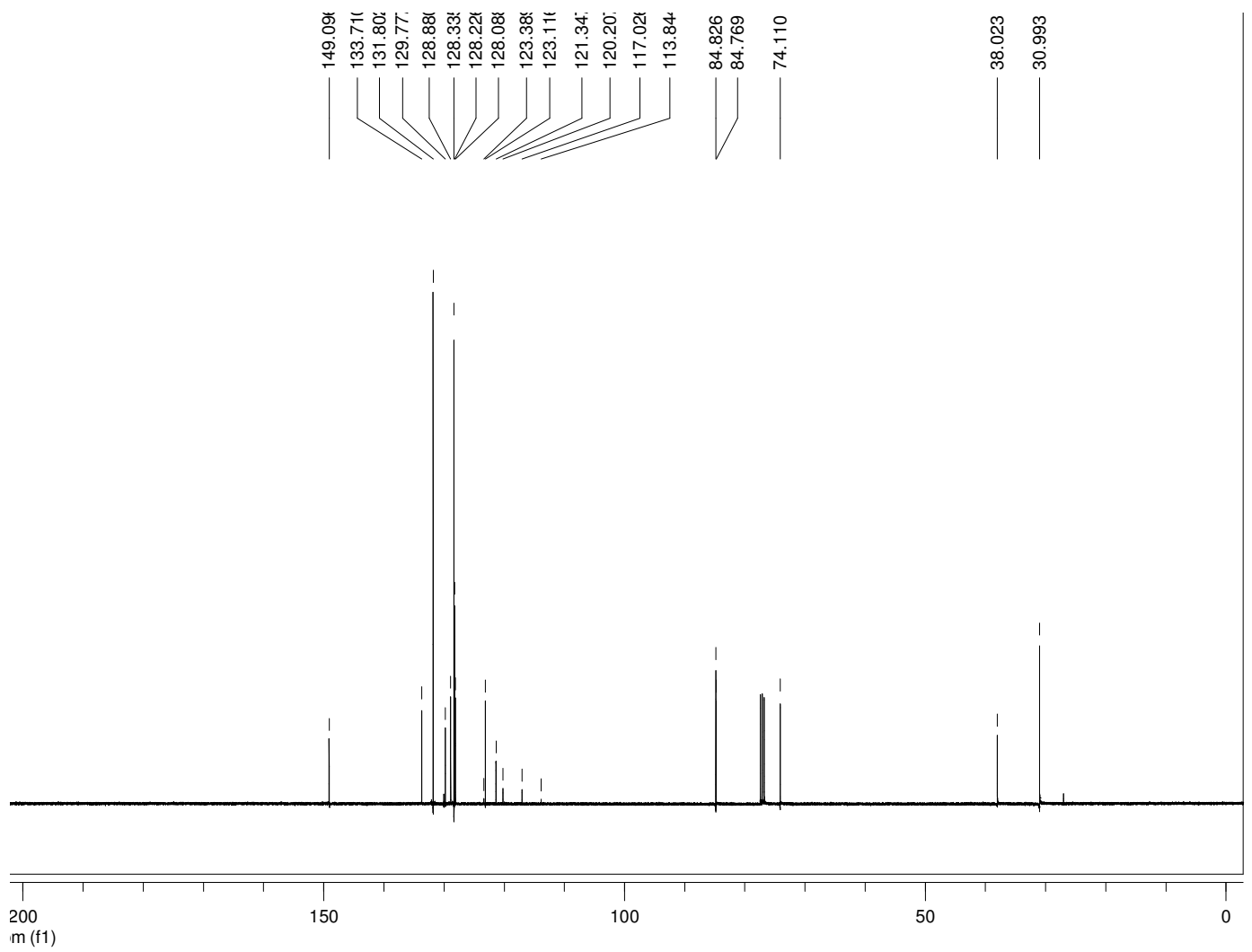
$(1 S, 3 R)-\mathrm{SI} 21\left({ }^{1} \mathrm{H}\right)$ :

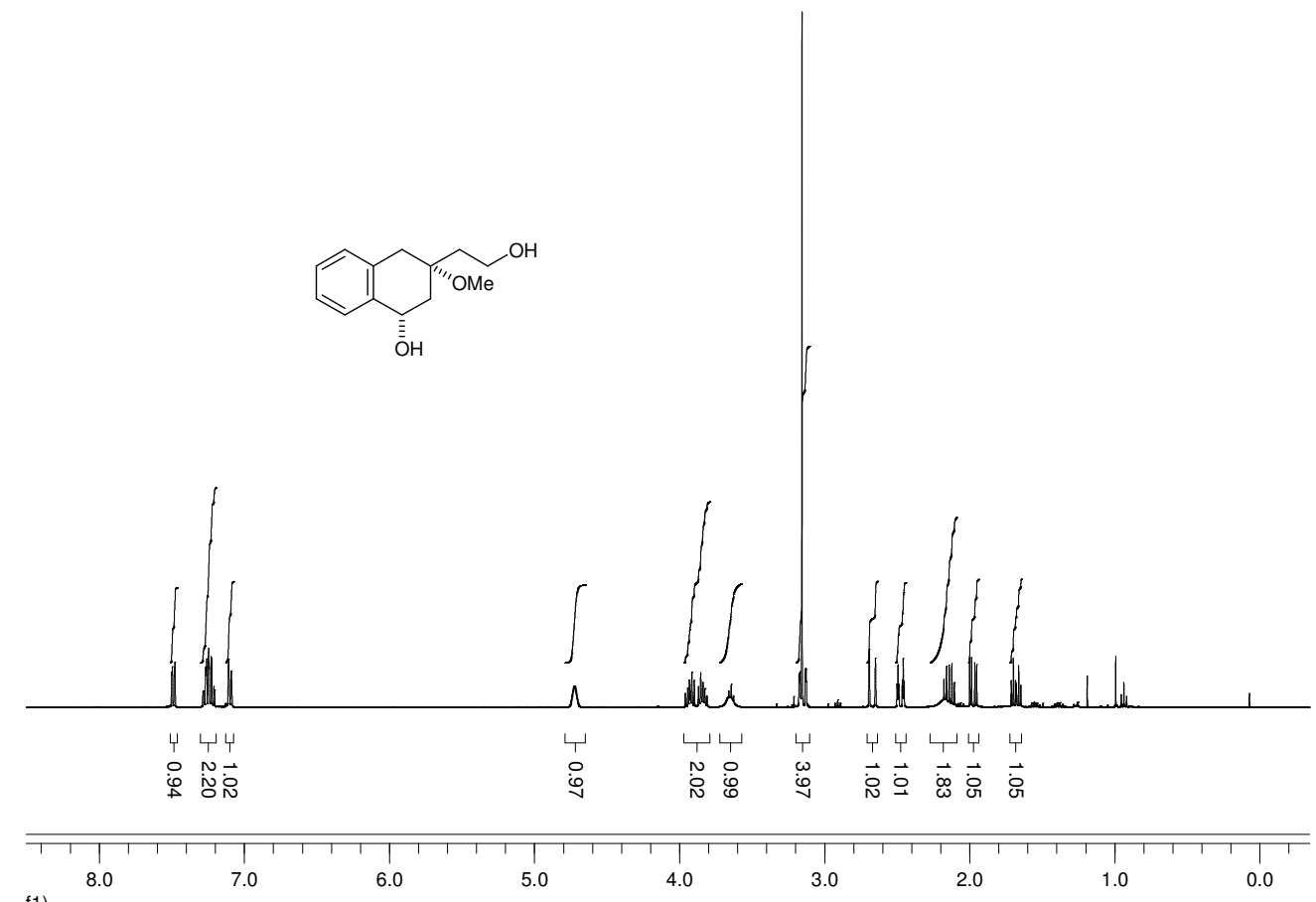

(1S,3R)-SI21 $\left({ }^{13} \mathrm{C}\right)$ :
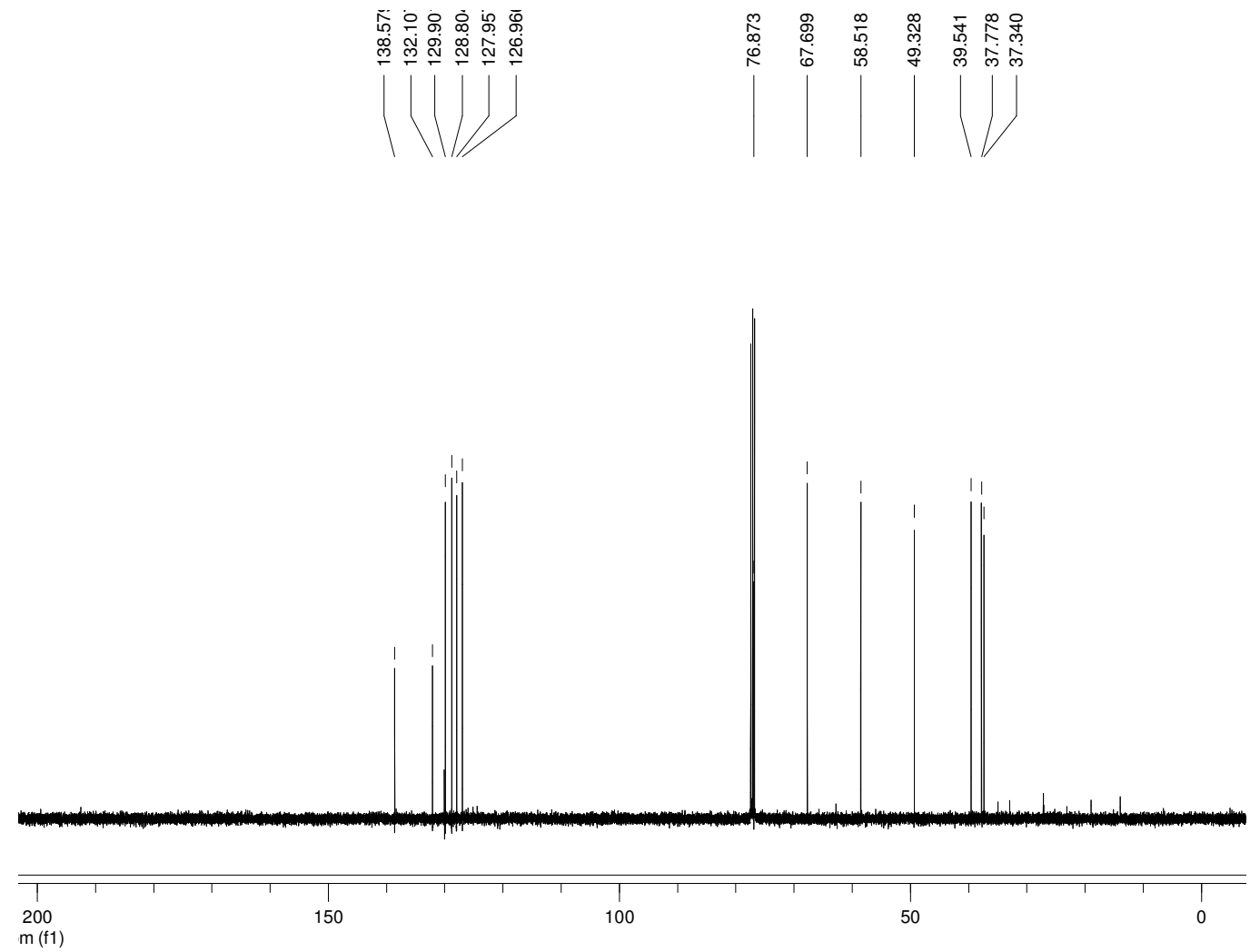
(1R,3R)-SI21 $\left({ }^{1} \mathrm{H}\right)$ :

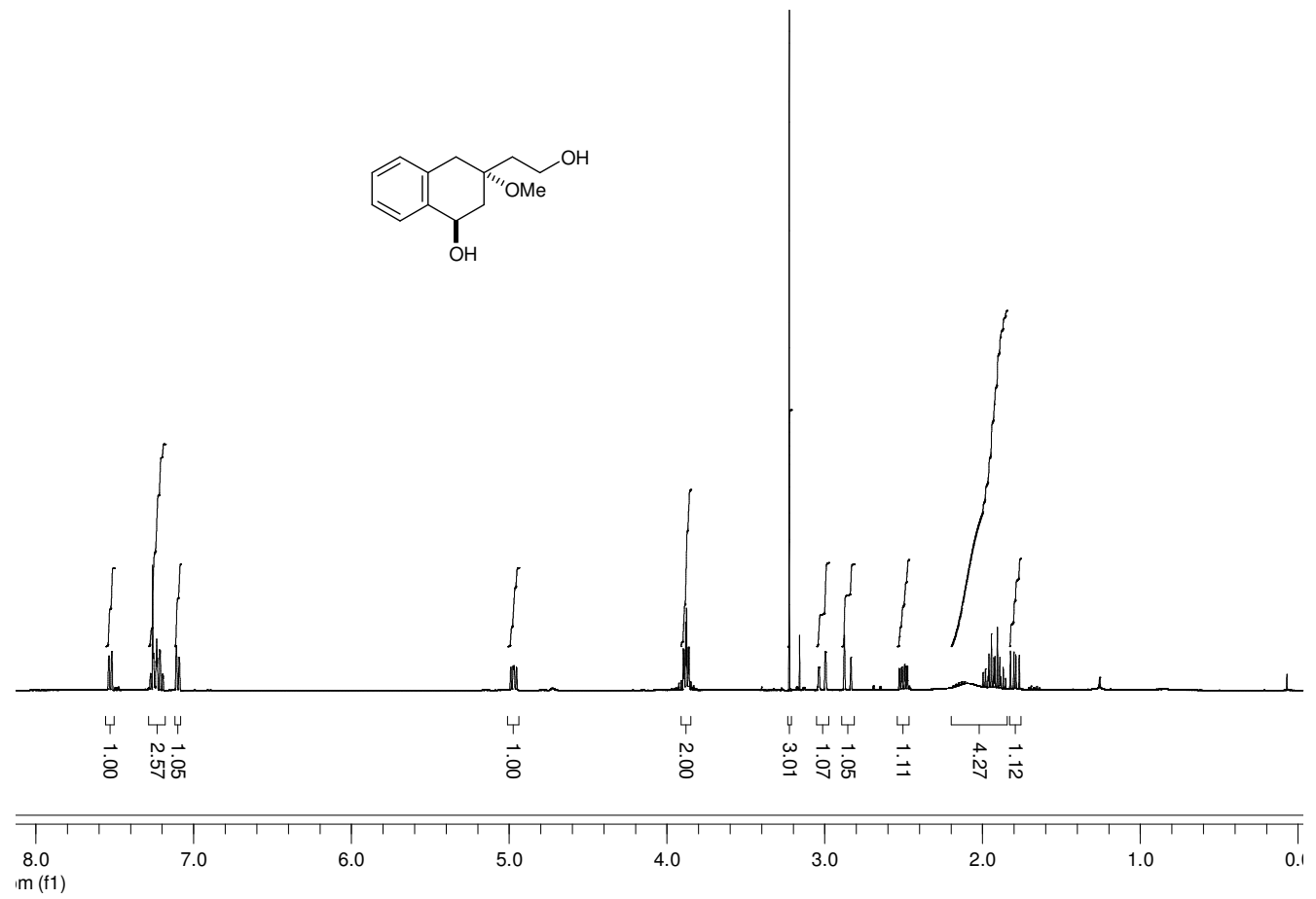

(1R,3R)-SI21 $\left({ }^{13} \mathrm{C}\right)$ :

충

悉
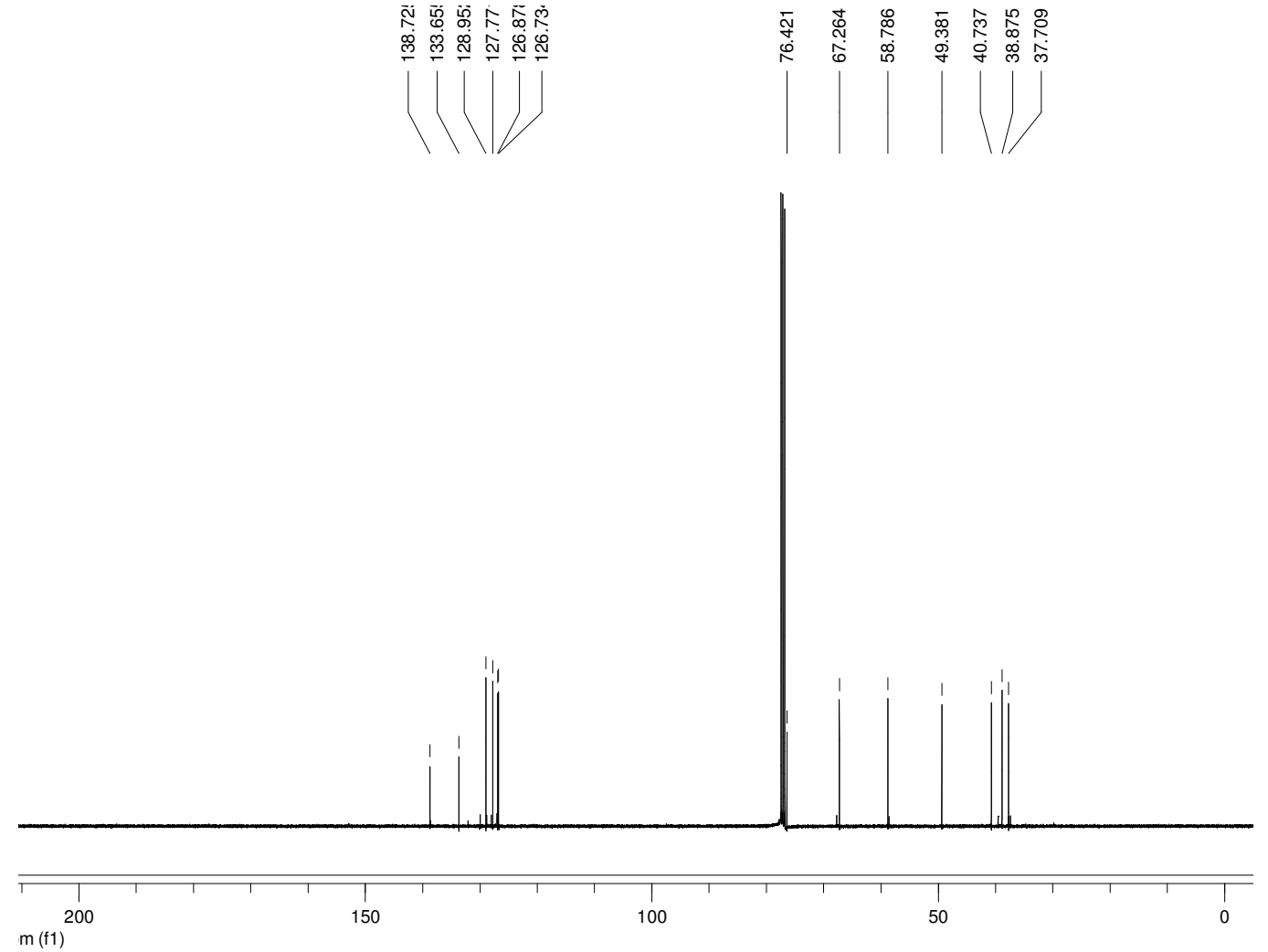
$(1 S, 3 R)-S \mid 22\left({ }^{1} \mathrm{H}\right)$ :

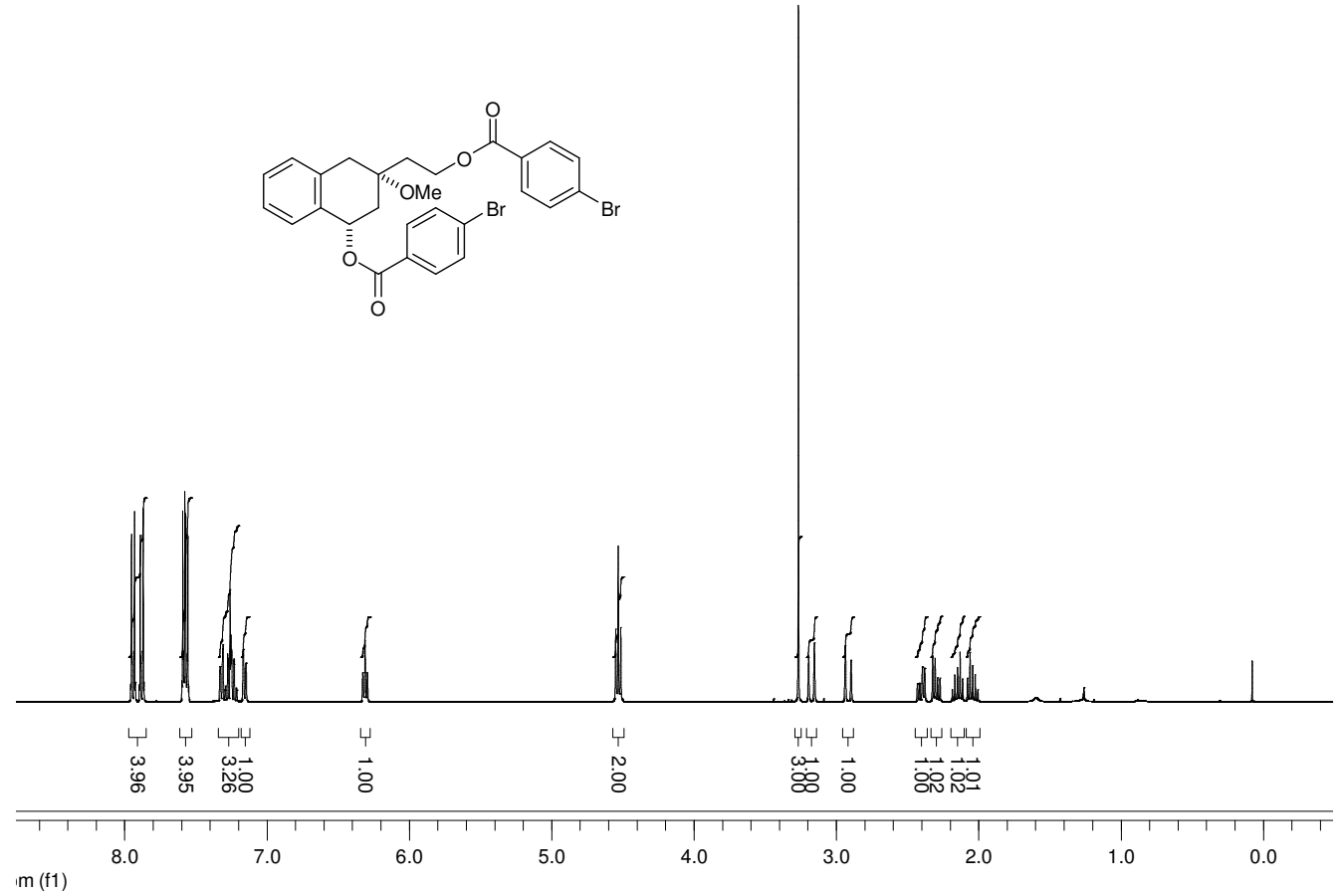

(1S,3R)-SI22 $\left({ }^{13} \mathrm{C}\right)$ :
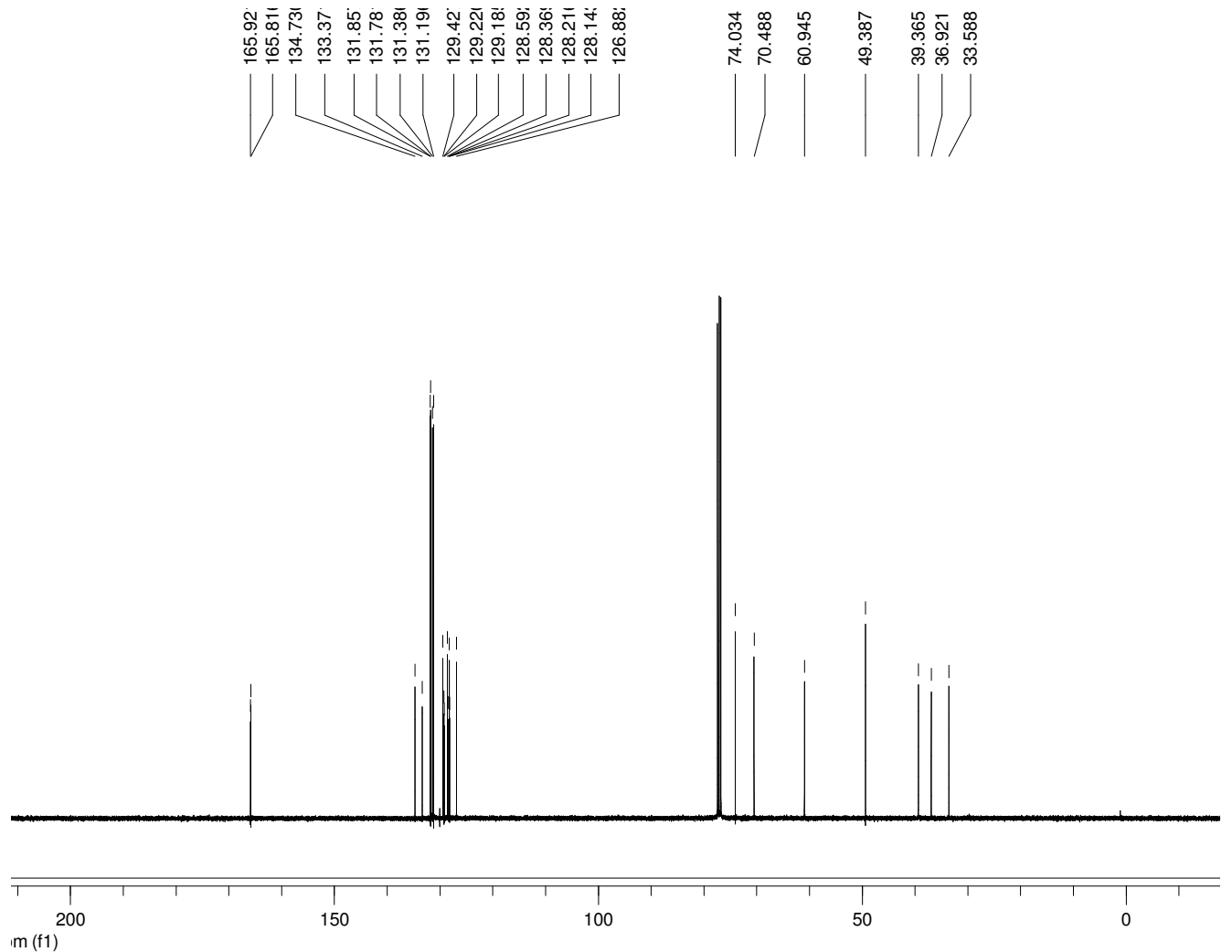


\section{Cartesian Coordinates and Energy Data of Model Complexes}

The B3LYP/LACV3P**++//B3LYP/LACVP** level of theory as implemented in the Jaguar 6.5 quantum chemistry program package has been utilized throughout this study. ${ }^{34}$ For O, C and $\mathrm{H}$, the 6-31G** basis set of Pople and coworkers was used for geometry optimizations. For palladium, a Hay-Wadt small effective core potential replaces the 28 innermost core electrons. Full structure optimizations and numerical vibrational frequency calculations have been performed for all model compounds at the B3LYP/LACVP** level. Stationary points are characterized by the absence of imaginary vibrations; transition structures are characterized by exactly one imaginary vibration. Visual inspection of imaginary vibrations was performed with the Molden program package. Single point energy calculations have been performed on the B3LYP/LACV3P**++ level of theory. The Gibbs free energies G refer to 298.15 $\mathrm{K}$ and $1 \mathrm{~atm}$ and are based on unscaled molecular vibrations and ideal gas phase conditions. 
M1<smiles>C[Pb]1PC=CC=CP1</smiles>

\section{NIMAG $\odot$}

$\begin{array}{lrrr}\text { C1 } & 4.5531320883 & -0.3451128720 & -2.4141104399 \\ \text { P2 } & 3.1707396316 & 0.7244344300 & -1.7720954066 \\ \text { C3 } & 3.0826758373 & 2.0334346885 & -3.0614561386 \\ \text { C4 } & 2.7283509526 & 1.9438948307 & -4.3592752851 \\ \text { C5 } & 2.3142809301 & 0.7979768334 & -5.1937491407 \\ \text { C6 } & 1.2946073583 & -0.0771165410 & -5.0828907362 \\ \text { P7 } & -0.0096492117 & -0.2094390425 & -3.7933180690 \\ \text { C8 } & -0.9567119153 & 1.3726626812 & -4.0530881762 \\ \text { C9 } & 4.0713861305 & 1.6782263944 & -0.4480593230 \\ \text { Pd10 } & 1.1148149716 & -0.3042391497 & -1.7892573976 \\ \text { C11 } & -1.1414964662 & -1.4213022381 & -4.6451825455 \\ \text { H12 } & 1.2096480305 & -0.8163087189 & -5.8810533815 \\ \text { H13 } & 2.9062014292 & 0.7196577082 & -6.1101100857 \\ \text { H14 } & 3.3814539312 & 3.0236510720 & -2.7139683825 \\ \text { H15 } & 2.8463526919 & 2.8597579903 & -4.9453712428 \\ \text { H16 } & 4.9916286265 & 2.1451604160 & -0.8175327178 \\ \text { H17 } & 3.4108816469 & 2.4520961291 & -0.0467636057 \\ \text { H18 } & 4.3215253201 & 0.9950983924 & 0.3684341092 \\ \text { H19 } & 5.4518999101 & 0.2393737048 & -2.6372707411 \\ \text { H20 } & 4.7917564568 & -1.1070271045 & -1.6665029825 \\ \text { H21 } & 4.2143304755 & -0.8493373456 & -3.3216765343 \\ \text { H22 } & -1.2570409466 & 1.5007763666 & -5.0983634914 \\ \text { H23 } & -1.8469947442 & 1.3654892410 & -3.4176870784 \\ \text { H24 } & -0.3266153076 & 2.2123977007 & -3.7519178687 \\ \text { H25 } & -1.4053960691 & -1.1080779233 & -5.6618886862 \\ \text { H26 } & -0.6584995631 & -2.4018217103 & -4.6844084714 \\ \text { H27 } & -2.0565809175 & -1.5223066568 & -4.0549631492\end{array}$

Energy components, in hartrees:

(A) Nuclear repulsion...........

980.64622972758

(E) Total one-electron terms..... $-3544.73121215774$

(I) Total two-electron terms..... 1440.12305968579

(J) Coulomb............... 1548.81002037278

(K) Exchange+Correlation.......

(L) Electronic energy........... $-2104.60815247195 \quad$ (E+I)

(N) Total energy............ $-1123.96192274437 \quad(\mathrm{~A}+\mathrm{L})$

SCFE: SCF energy: DFT $\quad-1123.96192274437$ hartrees

HOMO energy: $\quad-0.16069$

LUMO energy: $\quad-0.03649$

Thermodynamic properties calculated assuming an ideal gas

In the table below, the units for temperature

are kelvins, the units for $\mathrm{U}, \mathrm{H}$, and $\mathrm{G}$ are

$\mathrm{kcal} / \mathrm{mol}$ and the units for $\mathrm{CV}$ and $\mathrm{S}$ are $\mathrm{cal} /(\mathrm{mol} \mathrm{K})$

The zero point energy (ZPE): $138.052 \mathrm{kcal} / \mathrm{mol}$ is not included in $\mathrm{U}, \mathrm{H}$, or $\mathrm{G}$ in the table below

$\mathrm{T}=298.15 \mathrm{~K}$

\begin{tabular}{|c|c|c|c|c|c|}
\hline & U & CV & $\mathrm{S}$ & $\mathrm{H}$ & G \\
\hline trans. & 0.889 & 2.981 & 42.787 & 1.481 & -11.276 \\
\hline
\end{tabular}




$\begin{array}{rrrrrr}\text { rot. } & 0.889 & 2.981 & 31.942 & 0.889 & -8.635 \\ \text { vib. } & 8.373 & 51.939 & 53.637 & 8.373 & -7.619 \\ \text { elec. } & 0.000 & 0.000 & 0.000 & 0.000 & 0.000 \\ \text { total } & 10.150 & 57.901 & 128.366 & 10.743 & -27.530\end{array}$

Total internal energy, Utot (SCFE + ZPE + U): -1123.725728 hartrees Total enthalpy, Htot (Utot + pV): -1123.724784 hartrees Total Gibbs free energy, Gtot (Htot - $T^{\star} S$ ): -1123.785775 hartrees

Single point calculation at the B3LYP/LACV3P**++//B3LYP/LACVP** level

Energy components, in hartrees:

(A) Nuclear repulsion.......... 983.03068721461

(E) Total one-electron terms.... - -3549.28930547914

(I) Total two-electron terms..... 1442.16225071425

(J) Coulomb.............. 1550.85923694914

(K) Exchange+Correlation...... $\quad-108.69698623488$

(L) Electronic energy.......... -2107.12705476489 (E+I)

(N) Total energy............. $-1124.09636755028 \quad(\mathrm{~A}+\mathrm{L})$

SCFE: SCF energy: DFT $\quad-1124.09636755028$ hartrees

HOMO energy: -0.16827

LUMO energy: -0.05043

B3LYP/LACV3P**++//B3LYP/LACVP** Total Gibbs free energy, Gtot (Htot - $\left.T^{*} S\right)$ : -1123. 920219 hartrees

B3LYP/LACV3P**++//B3LYP/LACVP** Total Gibbs free energy, Gtot (Htot - $\mathrm{T}^{*} \mathrm{~S}$ ) of M1 and educt: $\quad-2406.529594$ hartrees 
M2

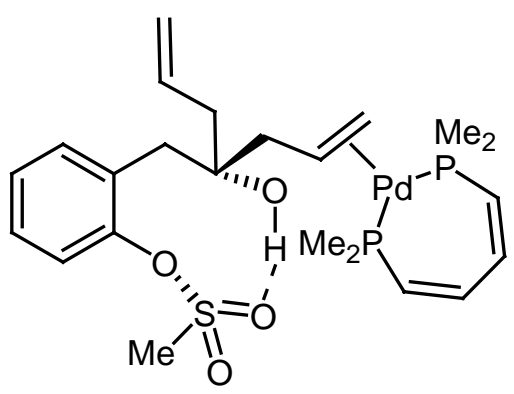

\section{NIMAG $\odot$}

\begin{tabular}{|c|c|c|c|}
\hline C1 & 2.3732852977 & -1.9369911335 & -0.2731093314 \\
\hline $\mathrm{C} 2$ & 1.4250604040 & -2.9179702458 & -0.5608938735 \\
\hline C3 & 0.0617222443 & -2.6356243673 & -0.4461064278 \\
\hline C4 & -0.3431641775 & -1.3608206807 & $-\odot .0558474924$ \\
\hline C5 & 0.6275474342 & -0.4032234807 & 0.2389346869 \\
\hline c6 & 2.0058693486 & -0.6508198880 & $\odot .1562898993$ \\
\hline 07 & ๑.1911473038 & 0.9126543770 & $\odot .5386011585$ \\
\hline c8 & 3.0337931651 & $\odot .4102967578$ & $\odot .4853963305$ \\
\hline c9 & 3.8137521404 & ๑. 2037220325 & 1.8311055117 \\
\hline C10 & 4.2346793306 & 1.6056119573 & 2.3578377607 \\
\hline C11 & 4.9988478385 & 1.5705034675 & 3.6524307086 \\
\hline C12 & 6.1465321650 & 2.2124198586 & 3.8861029340 \\
\hline 013 & 3.0238371257 & -0.4569332246 & 2.8119656936 \\
\hline C14 & 5.0424957623 & -0.7312625323 & 1.6646981644 \\
\hline C15 & 6.1287256079 & -0.2727283364 & $\odot .7120536561$ \\
\hline C16 & 6.2037734854 & $-\odot .6828647031$ & -0.6294651683 \\
\hline Pd17 & 7.6866367864 & -1.8178322032 & 0.5138355694 \\
\hline P18 & 8.6720157499 & -2.2911474377 & 2.6335379409 \\
\hline C19 & 8.9707952822 & -0.9178213312 & 3.8466556671 \\
\hline P20 & 8.8886967138 & -3.4949158653 & -0.6742921445 \\
\hline C21 & 8.2615203252 & -4.1866235108 & -2.2815833694 \\
\hline $\mathrm{C} 22$ & 10.6140898235 & -2.9941167105 & -1.1493462531 \\
\hline $\mathrm{C} 23$ & 9.1637449369 & -5.0594098961 & 0.2389646332 \\
\hline C24 & 9.8752221892 & -5.2734694461 & 1.3655820033 \\
\hline $\mathrm{C} 25$ & 10.6517730099 & -4.3324362772 & 2.1901311410 \\
\hline C26 & 10.2911989403 & -3.1493504585 & 2.7305713150 \\
\hline $\mathrm{C} 27$ & 7.5828947552 & -3.4263980938 & 3.6249486506 \\
\hline $\mathrm{S} 28$ & -0.4888262412 & 1.1850568905 & 2.0165869860 \\
\hline 029 & -1.7881605794 & ๑.5284301999 & 2.1043193778 \\
\hline 030 & 0.5136211675 & ๑.9455083799 & 3. 0611130160 \\
\hline C31 & -0.7027631670 & 2.9512523611 & 1.8033164777 \\
\hline H32 & -1.1590500306 & 3. 3203728632 & 2.7233298592 \\
\hline H33 & 0.2756658640 & 3.4056351035 & 1.6486754412 \\
\hline H34 & -1.3594708828 & 3.1222875583 & 0.9504596465 \\
\hline H35 & 3.4271324202 & -2.1689156741 & -0.3800233161 \\
\hline H36 & 1.7526197798 & -3.9030645950 & -0.8803828290 \\
\hline H37 & -0.6822034995 & -3.3938779749 & -0.6706833592 \\
\hline H38 & -1.3911305979 & -1.0959844038 & 0.0257189210 \\
\hline H39 & 3.7710312816 & 0.4674457853 & -0.3224027215 \\
\hline $\mathrm{H} 40$ & 2.5297038778 & 1.3800833414 & 0.5175494734 \\
\hline H41 & 2.1938061281 & ๑. 0301903576 & 2.9408859977 \\
\hline H42 & 4.8067666102 & 2.1415786055 & 1.5906652030 \\
\hline $\mathrm{H} 43$ & 3.3045192950 & 2.1737446355 & 2.5150879757 \\
\hline H44 & 5.4530991424 & $-\odot .8522958149$ & 2.6725207077 \\
\hline H45 & 4.6680978182 & -1.7167567639 & 1.3717016683 \\
\hline H46 & 4.5420940068 & ๑.9739448164 & 4. 4408441802 \\
\hline H47 & 6.6393577518 & 2.1673537499 & 4.8537669422 \\
\hline H48 & 6.6306143301 & 2.8153683349 & 3.1200807187 \\
\hline H49 & 6.6374284774 & 0.6489327711 & 0.9907736134 \\
\hline $\mathrm{H} 50$ & 6.7388747029 & $-\odot .0785226679$ & -1.3583668835 \\
\hline H51 & 5.4684627740 & -1.3661554424 & -1.0478768990 \\
\hline H52 & 11.0487383291 & -2.6324930538 & 3.3216212222 \\
\hline H53 & 11.6462706373 & -4.7029165963 & 2.4516460294 \\
\hline H54 & 8.6704367637 & -5.9283742717 & $-\odot .1986649296$ \\
\hline H55 & 9.9581018523 & -6.3115453900 & 1.6971791186 \\
\hline H56 & 8.9371861681 & -4.9293235282 & -2.7195432945 \\
\hline H57 & 7.2819212923 & -4.6440022556 & -2.1150927474 \\
\hline H58 & 8.1276160328 & -3.3620675866 & -2.9873416865 \\
\hline H59 & 11.1555987117 & -3.8075709686 & -1.6423631346 \\
\hline $\mathrm{H} 60$ & 10.5546438479 & -2.1336261406 & -1.8221131278 \\
\hline
\end{tabular}




$\begin{array}{rrrr}\text { H61 } & 11.1557543626 & -2.6912619809 & -0.2508715948 \\ \text { H62 } & 8.0039116234 & -3.6414391515 & 4.6121469784 \\ \text { H63 } & 6.6010032244 & -2.9585542250 & 3.7393856007 \\ \text { H64 } & 7.4549325228 & -4.3611191568 & 3.0742170876 \\ \text { H65 } & 9.2901149054 & -1.2837179846 & 4.8287670068 \\ \text { H66 } & 9.7363527139 & -0.2466071780 & 3.4461516841 \\ \text { H67 } & 8.0483977527 & -0.3407944833 & 3.9559026073\end{array}$

Energy components, in hartrees:
(A) Nuclear repulsion............
4083.11584637311
(E) Total one-electron terms..... -11481.56661775729
(I) Total two-electron terms.... 4991.83769027604
(J) Coulomb............. 5249.68033545860
(K) Exchange+Correlation....... -257.84264518256
(L) Electronic energy........... $-6489.72892748125 \quad(E+I)$
(N) Total energy............. $-2406.61308110814 \quad(\mathrm{~A}+\mathrm{L})$

SCFE: SCF energy: DFT $\quad-2406.61308110814$ hartrees

HOMO energy: -0.16812

LUMO energy: -0.04141

Thermodynamic properties calculated assuming an ideal gas

In the table below, the units for temperature

are kelvins, the units for $\mathrm{U}, \mathrm{H}$, and $\mathrm{G}$ are

$\mathrm{kcal} / \mathrm{mol}$ and the units for $\mathrm{Cv}$ and $\mathrm{S}$ are $\mathrm{cal} /(\mathrm{mol} \mathrm{K})$

The zero point energy (ZPE): $344.422 \mathrm{kcal} / \mathrm{mol}$

is not included in $U, H$, or $G$ in the table below

$T=298.15 \mathrm{~K}$

\begin{tabular}{rrrrrr} 
& \multicolumn{1}{c}{ U } & \multicolumn{1}{c}{ CV } & \multicolumn{1}{c}{ S } & \multicolumn{1}{c}{ H } & \multicolumn{1}{c}{ G } \\
trans. & 0.889 & 2.981 & 44.938 & 1.481 & -11.917 \\
rot. & 0.889 & 2.981 & 37.784 & 0.889 & -10.377 \\
vib. & 22.124 & 135.776 & 151.582 & 22.124 & -23.070 \\
elec. & 0.000 & 0.000 & 0.000 & 0.000 & 0.000 \\
total & 23.902 & 141.738 & 234.304 & 24.494 & -45.364
\end{tabular}

Total internal energy, Utot (SCFE + ZPE + U): $\quad-2406.026120$ hartrees Total enthalpy, Htot (Utot + pV): -2406.025175 hartrees Total Gibbs free energy, Gtot (Htot - $T^{\star} S$ ): $\quad-2406.136501$ hartrees

\section{Single point calculation at the B3LYP/LACV3P**++//B3LYP/LACVP** level}

Energy components, in hartrees:
(A) Nuclear repulsion............
4083.11584637311
(E) Total one-electron terms ..... -11480.77119887026
(I) Total two-electron terms.... 4990.64389513622
(J) Coulomb............. 5248.54330849964
(K) Exchange+Correlation...... $\quad-257.89941336341$
(L) Electronic energy.......... -6490.12730373404
(N) Total energy............. $-2407.01145736093 \quad(\mathrm{~A}+\mathrm{L})$

SCFE: SCF energy: DFT $\quad-2407.01145736093$ hartrees

HOMO energy: $\quad-0.17873$

LUMO energy: $\quad-0.05684$

B3LYP/LACV3P**++//B3LYP/LACVP** Total Gibbs free energy, Gtot (Htot - T*S): -2406.534877 hartrees 
M3

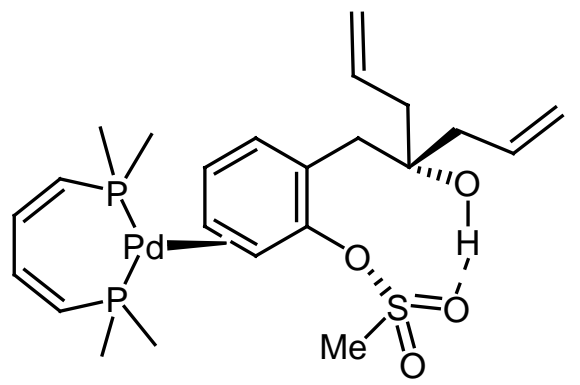

NIMAG $\odot$

\begin{tabular}{|c|c|c|c|}
\hline $\mathrm{C} 1$ & 1.5352155273 & 0.2997922326 & $\odot .1428295864$ \\
\hline C2 & 2.6609541125 & $-\odot .5425197024$ & $-\odot .0949725672$ \\
\hline C3 & 2.5219642272 & -1.8842685661 & -0.3838225983 \\
\hline C4 & 1.2347519625 & -2.4799942062 & -0.4902949640 \\
\hline C5 & ๑. 0879088933 & -1.6591440173 & -0.2977375788 \\
\hline c6 & ๑. 2871314129 & -0.2904143949 & ๑. 0369858659 \\
\hline $\mathrm{Pd} 7$ & $\odot .2731357209$ & -2.0439580551 & -2.5559751702 \\
\hline P8 & 1.1275339961 & -3.2142127067 & -4.4273697493 \\
\hline c9 & 1.2990761162 & -2.2324356368 & -5.9587534971 \\
\hline C10 & $\odot .3465334099$ & -1.6121170704 & -6.6906944316 \\
\hline C11 & -1.1079656207 & -1.5329101106 & -6.4941681226 \\
\hline C12 & -1.8267788781 & -1.1781110309 & -5.4058020963 \\
\hline P13 & -1.2399888817 & -0.6477258985 & -3.7568904615 \\
\hline C14 & $-\odot .3387738549$ & 0.9334545488 & -4.1420197742 \\
\hline 015 & $-\odot .8584581861$ & $\odot .5164931533$ & 0.1978398452 \\
\hline S16 & -1.8843467064 & $\odot .1343695469$ & 1.4523890354 \\
\hline C17 & -2.4114642380 & 1.8073630330 & 1.8161768648 \\
\hline C18 & 1.7352229421 & 1.7775547748 & 0.4125534539 \\
\hline C19 & 1.8607342929 & 2.2300197303 & 1.8987303538 \\
\hline C20 & 3.0589961780 & 1.5762803272 & 2.6379037357 \\
\hline C21 & 4.4336686809 & 1.9736350291 & 2.1706430157 \\
\hline $\mathrm{C} 22$ & 5.3965934794 & 1.1252121145 & 1.8023115971 \\
\hline C23 & $-2.827870 \odot 437$ & $-\odot .0019231363$ & -3.0434767426 \\
\hline 024 & -3.0025563478 & -0.6404583425 & 0.9227578031 \\
\hline 025 & -1.0857541432 & -0.3980858962 & 2.5606376011 \\
\hline C26 & 1.9392501603 & 3.7769480377 & 1.9161756834 \\
\hline C27 & 2.0712389825 & 4.3795513660 & 3.2887694454 \\
\hline $\mathrm{C} 28$ & 2.9959794802 & 5.2746792230 & 3.6415202244 \\
\hline 029 & ๑.6687463480 & 1.9249587355 & 2.6279080582 \\
\hline C30 & 2.7531177038 & -4.1189187509 & -4.4499943283 \\
\hline C31 & -0.0368911593 & -4.5720555498 & -4.9341085527 \\
\hline H32 & -3.1685039501 & 1.7370671480 & 2.5992628307 \\
\hline H33 & -1.5270244403 & 2.3488766439 & 2.1545897017 \\
\hline H34 & -2.8359799744 & 2.2374600919 & 0.9086592677 \\
\hline H35 & 3.6500968627 & -๑. 0988454191 & -0.0411070621 \\
\hline H36 & 3.4045559959 & -2.5018547518 & -0.5282291210 \\
\hline H37 & 1.1375287440 & -3.5607503958 & -0.4797662963 \\
\hline H38 & -0.8933636464 & -2.0946051191 & -0.1412341688 \\
\hline H39 & 2.6454792765 & 2.0968451779 & -0.1078939148 \\
\hline H4O & 0.8999844468 & 2.3389032644 & -0.0183558903 \\
\hline H41 & $\odot .5502493091$ & $\odot .9635852924$ & 2.6940535698 \\
\hline H42 & 2.7618407562 & 4.1122658882 & 1.2751231755 \\
\hline H43 & 1.0094155068 & 4.1376043745 & 1.4538164253 \\
\hline H44 & 2.9517823716 & 1.8620549384 & 3.6924490693 \\
\hline H45 & 2.9504275466 & 0.4861913147 & 2.5865494497 \\
\hline H46 & 1.3371884396 & 4.0436608580 & 4.0193717585 \\
\hline H47 & $3.03787 \odot 2144$ & 5.6889061526 & 4.6449905918 \\
\hline H48 & 3.7432248999 & 5.6330294204 & 2.9357451365 \\
\hline H49 & 4.6546459239 & 3.0404406100 & 2.1858089878 \\
\hline H50 & 6.3838659693 & 1.4741165676 & 1.5123046674 \\
\hline H51 & 5.2350995844 & 0.0496852277 & 1.7929550616 \\
\hline H52 & -2.9121819638 & -1.1964119387 & -5.5128963231 \\
\hline H53 & -1.6788464874 & -1.7248010529 & -7.4061526983 \\
\hline H54 & 2.3247914600 & -2.1417864311 & -6.3185144255 \\
\hline H55 & 0.6824012343 & -1.1495723259 & -7.6221918249 \\
\hline H56 & 2.9295855955 & -4.6560470680 & -5.3880893179 \\
\hline H57 & 3.5651345628 & -3.4050740065 & -4.2841179683 \\
\hline H58 & 2.7633705132 & -4.8337671671 & -3.6223014722 \\
\hline H59 & $\odot .2939928904$ & -5.0735872053 & -5.8489133196 \\
\hline H60 & -0.1023692868 & -5.3007042066 & -4.1207967728 \\
\hline H61 & -1.0296070010 & -4.1466723235 & -5.0935351641 \\
\hline
\end{tabular}




$\begin{array}{lrrr}\text { H62 } & -0.9560441483 & 1.6263472838 & -4.7226789158 \\ \mathrm{H} 63 & -0.0443052008 & 1.4038863810 & -3.1996505229 \\ \mathrm{H} 64 & 0.5665349415 & 0.6951386278 & -4.7045381429 \\ \mathrm{H} 65 & -3.2856652961 & 0.7740749639 & -3.6668891454 \\ \mathrm{H} 66 & -3.5321621928 & -0.8292043047 & -2.9192581161 \\ \mathrm{H} 67 & -2.6185914852 & 0.4010640927 & -2.0496612760\end{array}$

Energy components, in hartrees:
(A) Nuclear repulsion............
4234.05223977471
(E) Total one-electron terms.....
$-11783.20453109884$
(I) Total two-electron terms.....
5142.55048528835
(J) Coulomb.............. 5400.39584709679
(K) Exchange+Correlation....... -257.84536180844
(L) Electronic energy.......... -6640.65404581049
(N) Total energy............. -2406.60180603578

SCFE: SCF energy: DFT $\quad-2406.60180603578$ hartrees

HOMO energy: -0.15283

LUMO energy: $-\odot .04252$

Thermodynamic properties calculated assuming an ideal gas

In the table below, the units for temperature

are kelvins, the units for $\mathrm{U}, \mathrm{H}$, and $\mathrm{G}$ are

$\mathrm{kcal} / \mathrm{mol}$ and the units for $\mathrm{Cv}$ and $\mathrm{S}$ are $\mathrm{cal} /(\mathrm{mol} \mathrm{K})$

The zero point energy (ZPE): $343.895 \mathrm{kcal} / \mathrm{mol}$

is not included in $\mathrm{U}, \mathrm{H}$, or $\mathrm{G}$ in the table below

$\mathrm{T}=298.15 \mathrm{~K}$

\begin{tabular}{|c|c|c|c|c|c|}
\hline & U & CV & $\mathrm{S}$ & H & G \\
\hline trans. & ๑.889 & 2.981 & 44.938 & 1.481 & -11.917 \\
\hline rot. & 0.889 & 2.981 & 37.444 & 0.889 & -10.275 \\
\hline vib. & 22.459 & 136.699 & 154.187 & 22.459 & -23.512 \\
\hline elec. & 0.000 & $0.0 \odot \odot$ & 0.000 & 0.000 & $\odot .0 \odot \odot$ \\
\hline total & 24.237 & 142.661 & 236.569 & 24.829 & -45.704 \\
\hline
\end{tabular}

Total internal energy, Utot (SCFE + ZPE + U): $\quad-2406.015150$ hartrees Total enthalpy, Htot (Utot + pV): -2406.014206 hartrees Total Gibbs free energy, Gtot (Htot - $T^{\star} S$ ): $\quad-2406.126607$ hartrees

\section{Single point calculation at the B3LYP/LACV3P**++//B3LYP/LACVP** level}

Energy components, in hartrees:
(A) Nuclear repulsion............
4234.05223977471
(E) Total one-electron terms.....
$-11782.41375835384$
(I) Total two-electron terms.....
5141.36354095338

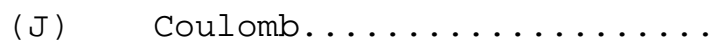
5399.26710924343
(K) Exchange+Correlation........
$-257.90356829005$
(L) Electronic energy........... -6641.05021740046
(N) Total energy............. -2406.99797762575

SCFE: SCF energy: DFT $\quad-2406.99797762575$ hartrees

HOMO energy: $-\odot .16400$

LUMO energy: -0.05803

B3LYP/LACV3P**++//B3LYP/LACVP** Total Gibbs free energy, Gtot (Htot - T*S): -2406.522778 hartrees 
M4

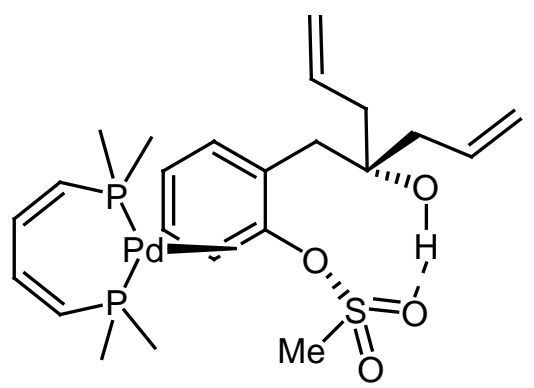

\section{NIMAG $\odot$}

\begin{tabular}{|c|c|c|c|}
\hline C1 & 1.7207577273 & -2.3763409694 & 0.9177907662 \\
\hline $\mathrm{C} 2$ & 0.6205274955 & -3.2499072196 & 1.0229746598 \\
\hline C3 & -0.6508744687 & -2.8135420959 & 0.7077535898 \\
\hline C4 & $-\odot .8563967498$ & -1.4911339760 & $\odot .2434020425$ \\
\hline C5 & 0.2677812316 & -0.6252142160 & 0.1556071944 \\
\hline $\mathrm{C} 6$ & 1.5795133470 & -1.0483005164 & $\odot .5348756066$ \\
\hline $\mathrm{Pd} 7$ & -0.2923212168 & -1.3303070573 & -2.0235011734 \\
\hline P8 & -1.9214495566 & -2.2736519663 & -3.4823184012 \\
\hline C9 & -3.1053494047 & -3.6354596719 & -3.0248537140 \\
\hline 010 & 0.0144945078 & 0.7750966273 & $-\odot .0330722255$ \\
\hline S11 & -0.6020713971 & 1.6465819057 & 1.2284802580 \\
\hline 012 & -1.9282353869 & 1.1596347157 & 1.5950642427 \\
\hline C13 & 2.7616299595 & -0.1039502414 & 0.4422987763 \\
\hline C14 & 3.5259518692 & 0.2275829112 & 1.7724004351 \\
\hline C15 & 4.1294213571 & 1.6552865634 & 1.6127933136 \\
\hline C16 & 4.9619581472 & 2.1087452078 & 2.7773465471 \\
\hline C17 & 6.1839090599 & 2.6365662436 & 2. 6915173861 \\
\hline 018 & 0.4181860798 & 1.8019317227 & 2.2748003918 \\
\hline C19 & -0.7420320714 & 3.1912829979 & 0.3316552079 \\
\hline P20 & 1.2078650183 & -0.5801041686 & -3.7126393746 \\
\hline C21 & 2.3949055359 & 0.8407809762 & -3.5178672148 \\
\hline $\mathrm{C} 22$ & 2.3762227390 & -1.9494470902 & -4.1780673247 \\
\hline $\mathrm{C} 23$ & 0.4793657495 & -0.1311088773 & -5.3283870592 \\
\hline C24 & -0.2408432380 & -0.8901834633 & -6.1823540983 \\
\hline $\mathrm{C} 25$ & -0.6767166423 & -2.2878726988 & -6.0771446639 \\
\hline C26 & -1.3232435853 & -2.9297085372 & -5.0802290774 \\
\hline 027 & 2.6866906025 & 0.1898048080 & 2.9153333614 \\
\hline C28 & 4.6220151617 & -0.8201354703 & 2.1063970289 \\
\hline C29 & 5.7094341725 & $-\odot .9856185944$ & 1.0845409225 \\
\hline C30 & 5.9533710751 & -2.1021059555 & 0.3939682496 \\
\hline C31 & -3.1128217948 & -0.9487398680 & -4.0181544251 \\
\hline H33 & -1.1487736421 & 3.9204499184 & 1.0336175845 \\
\hline H34 & 0.2496397412 & 3.4915220877 & $-\odot .0062276139$ \\
\hline H35 & -1.4198861051 & 3.0430849156 & -0.5084448941 \\
\hline H36 & 2.7108173515 & -2.7516202028 & 1.1509552869 \\
\hline H37 & 0.7785534064 & -4.2675758328 & 1.3686207388 \\
\hline H38 & -1.5088419008 & -3.4696827188 & ๑.8231681777 \\
\hline H39 & -1.8539715621 & -1.0707690019 & 0.1980454963 \\
\hline H4O & 3.4936506550 & $-\odot .5150889692$ & -0.2634514228 \\
\hline H41 & 2.4100010425 & 0.8351913552 & 0.0076993342 \\
\hline H42 & 1.9023524259 & $\odot .7396937087$ & 2.7537198448 \\
\hline H43 & 4.7079202765 & 1.7216352924 & 0.6824126970 \\
\hline H44 & 3.2730861971 & 2.3369442995 & 1.5006816404 \\
\hline H45 & 5.0618759755 & -0.5031824325 & 3.0600872848 \\
\hline H46 & 4.1280153915 & -1.7766714250 & 2.2994179626 \\
\hline H47 & 4.4938408353 & 1.9863354315 & 3.7531088475 \\
\hline H48 & 6.7304680975 & 2.9565617859 & 3.5738833501 \\
\hline H49 & 6.6833690716 & 2.7768585690 & 1.7346748321 \\
\hline H50 & 6.3518123829 & -0.1190344552 & 0.9230145297 \\
\hline H51 & 6.7691605699 & -2.1660898435 & $-\odot .3210017599$ \\
\hline H52 & 5.3544592271 & -3.0009489750 & 0.5274350641 \\
\hline H53 & -1.5371543560 & -3.9868133638 & -5.2401294517 \\
\hline H54 & -0.5022473403 & -2.8649465052 & -6.9885250628 \\
\hline H55 & 0.6768746576 & 0.8954674517 & -5.6386925685 \\
\hline H56 & -0.4882217392 & -0.4308434579 & -7.1424838185 \\
\hline H57 & 3.0551214603 & 0.9612486299 & -4.3832208358 \\
\hline H58 & 1.8295085488 & 1.7644957092 & -3.3664181405 \\
\hline H59 & 3.0024641409 & $\odot .6714459199$ & -2.6252338986 \\
\hline H60 & 3.0440593752 & -1.6541107763 & -4.9931287924 \\
\hline H61 & 2.9683880836 & -2.2216956964 & -3.3002013321 \\
\hline
\end{tabular}




$\begin{array}{llll}\text { H62 } & 1.7954927278 & -2.8211922703 & -4.4853612197 \\ \text { H63 } & -3.8373696757 & -1.3236342407 & -4.7478931200 \\ \text { H64 } & -3.6434272991 & -0.5738534302 & -3.1382392702 \\ \text { H65 } & -2.5513245107 & -0.1231944291 & -4.4595505195 \\ \text { H66 } & -3.8349819817 & -3.8451472738 & -3.8143049528 \\ \text { H67 } & -2.5409092971 & -4.5462587630 & -2.8062774432 \\ \text { H68 } & -3.6389661057 & -3.3460916015 & -2.1150237813\end{array}$

Energy components, in hartrees:

(A) Nuclear repulsion......... 4293.43296784596

(E) Total one-electron terms..... -11901.95060321609

(I) Total two-electron terms..... 5201.92843739174

(J) Coulomb.............. 5459.77000813883

(K) Exchange+Correlation...... $\quad-257.84157074709$

(L) Electronic energy........... -6700.02216582435

(N) Total energy ........... $-2406.58919797838 \quad(\mathrm{~A}+\mathrm{I})$

SCFE: SCF energy: DFT $\quad-2406.58919797838$ hartrees

HOMO energy: $-\odot .15434$

LUMO energy: $-\odot .04550$

Thermodynamic properties calculated assuming an ideal gas

In the table below, the units for temperature

are kelvins, the units for $\mathrm{U}, \mathrm{H}$, and $\mathrm{G}$ are

$\mathrm{kcal} / \mathrm{mol}$ and the units for $\mathrm{Cv}$ and $\mathrm{S}$ are $\mathrm{cal} /(\mathrm{mol} \mathrm{K})$

The zero point energy (ZPE): $\quad 343.270 \mathrm{kcal} / \mathrm{mol}$

is not included in $\mathrm{U}, \mathrm{H}$, or $\mathrm{G}$ in the table below

$\mathrm{T}=298.15 \mathrm{~K}$

\begin{tabular}{rrrrrr} 
& \multicolumn{1}{c}{ U } & \multicolumn{1}{c}{ CV } & \multicolumn{1}{c}{ S } & \multicolumn{1}{c}{ H } & \multicolumn{1}{c}{ G } \\
trans. & 0.889 & 2.981 & 44.938 & 1.481 & -11.917 \\
rot. & 0.889 & 2.981 & 37.228 & 0.889 & -10.211 \\
vib. & 22.852 & 137.336 & 160.341 & 22.852 & -24.954 \\
elec. & 0.000 & 0.000 & 0.000 & 0.000 & 0.000 \\
total & 24.629 & 143.298 & 242.507 & 25.222 & -47.082
\end{tabular}

Total internal energy, Utot (SCFE + ZPE + U): -2406.002932 hartrees Total enthalpy, Htot (Utot + pV): -2406.001988 hartrees

Total Gibbs free energy, Gtot (Htot - $T^{\star} S$ ): $\quad-2406.117211$ hartrees

\section{Single point calculation at the B3LYP/LACV3P**++//B3LYP/LACVP** level}

Energy components, in hartrees:

(A) Nuclear repulsion.......... 4293.50643672794

(E) Total one-electron terms..... -11901.22886965877

(I) Total two-electron terms..... 5200.73430302761

(J) Coulomb............. 5458.63612121342

(K) Exchange+Correlation....... -257.90181818580

(L) Electronic energy........... $-6700.49456663116 \quad(\mathrm{E}+\mathrm{I})$

(N) Total energy............. -2406.98812990322 (A+L)

SCFE: SCF energy: DFT $\quad-2406.98812990322$ hartrees

HOMO energy: -0.16551

LUMO energy: -0.06091

B3LYP/LACV3P**++//B3LYP/LACVP** Total Gibbs free energy, Gtot (Htot - T*S): -2406.516143 hartrees 
M5

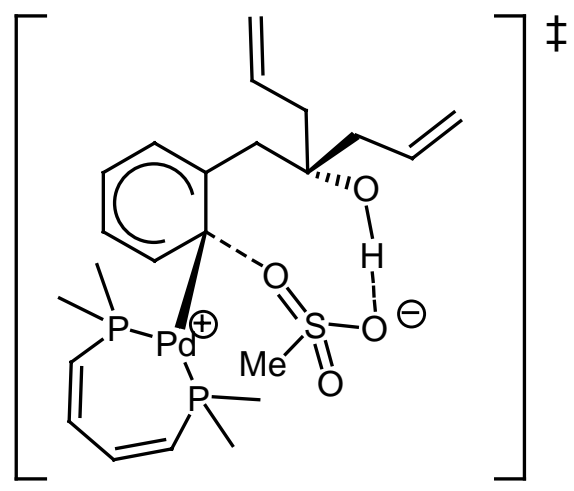

\section{NIMAG 1}

\begin{tabular}{|c|c|c|c|}
\hline C1 & 1.0716222000 & -3.2397392000 & 1.0564549000 \\
\hline $\mathrm{C} 2$ & $-\odot .0421058000$ & -2.9307936000 & $\odot .2926345000$ \\
\hline C3 & -0.0508331000 & -1.7325257000 & -0.4575693000 \\
\hline $\mathrm{C} 4$ & 1.1127418000 & -0.9344737000 & -0.4631036000 \\
\hline C5 & 2.2172888000 & -1.1619284000 & 0.3783366000 \\
\hline C6 & 2.1708942000 & -2.3627612000 & 1.0951307000 \\
\hline 07 & $\odot .4750911000$ & 1.1374791000 & $-\odot .200690000 \odot$ \\
\hline S8 & -0.5943314000 & 1.1916589000 & 0.9013797000 \\
\hline 09 & -0.0777000000 & $\odot .6134753000$ & 2.1697818000 \\
\hline Pd10 & $\odot .851555100 \odot$ & $-\odot .871806200 \odot$ & -2.4590629000 \\
\hline P11 & 1.6388957000 & 1.1981002000 & -3.3031585000 \\
\hline C12 & 2.9900986000 & 2.1371160000 & -2.4604045000 \\
\hline C13 & 3.4305946000 & $-\odot .268639700 \odot$ & ๑. 4989548000 \\
\hline C14 & 3.4937044000 & 0.6044488000 & 1.8217284000 \\
\hline 015 & 2.6289054000 & 0.1078603000 & 2.8252826000 \\
\hline C16 & -0.7800076000 & 2.9658954000 & 1.1982780000 \\
\hline 017 & -1.9047658000 & ๑. 6945173000 & $\odot .4387936000$ \\
\hline C18 & 4.9032463000 & $\odot .4945625000$ & 2.4654491000 \\
\hline C19 & 6.0520536000 & $\odot .9713050000$ & 1.6225650000 \\
\hline $\mathrm{C} 20$ & 7.0390690000 & ๑. 1984814000 & 1.1622556000 \\
\hline $\mathrm{C} 21$ & 3.1501730000 & 2.0810515000 & 1.4814375000 \\
\hline $\mathrm{C} 22$ & 3.0762440000 & 2.9879857000 & 2.6784737000 \\
\hline C23 & 3.6540169000 & 4.1888412000 & 2.7679935000 \\
\hline P24 & $\odot .1739468000$ & -1.6594594000 & -4.6261036000 \\
\hline C25 & $-\odot .9471040000$ & $-\odot .6053540000$ & -5.6574667000 \\
\hline C26 & 1.6826190000 & -1.8769039000 & -5.6223206000 \\
\hline $\mathrm{C} 27$ & 2.5398197000 & -0.9400808000 & -6.0742558000 \\
\hline C28 & 2.4996094000 & $\odot .5283652000$ & -6.0176788000 \\
\hline C29 & 2.1616286000 & 1.4053152000 & -5.0504223000 \\
\hline C30 & $-\odot .6302232000$ & -3.3179456000 & -4.7611319000 \\
\hline C31 & $\odot .261241200 \odot$ & 2.4268268000 & -3.2148035000 \\
\hline H32 & -1.5313865000 & 3. 0933390000 & 1.9797608000 \\
\hline H33 & $\odot .176127400 \odot$ & 3. 3777276000 & 1.5254983000 \\
\hline H34 & -1.1130531000 & 3.4480393000 & ๑. 2777699000 \\
\hline H35 & 3.0349898000 & -2.6210846000 & 1. 7010998000 \\
\hline H36 & $1.085352200 \odot$ & -4.1373663000 & 1.6673798000 \\
\hline H37 & -0.9427573000 & -3.5362099000 & 0.3416051000 \\
\hline H38 & $-\odot .9996314000$ & -1.3247208000 & -0.7979494000 \\
\hline H39 & 4.3200218000 & -0.9069402000 & $\odot .4722995000$ \\
\hline $\mathrm{H} 40$ & 3.4967563000 & ๑. 3824589000 & -0.3738083000 \\
\hline H41 & 1.6953113000 & $\odot .2385763000$ & 2.5554942000 \\
\hline $\mathrm{H} 42$ & 3.8790382000 & 2.4721312000 & $\odot .7603794000$ \\
\hline H43 & 2.1812980000 & 2.0656052000 & $\odot .9697549000$ \\
\hline H44 & 4.8633182000 & 1.0793595000 & 3. 3930261000 \\
\hline H45 & 5.0534428000 & -0.5525820000 & 2.7523375000 \\
\hline H46 & 2.4880959000 & 2.6079923000 & 3.5122362000 \\
\hline $\mathrm{H} 47$ & 3.5477343000 & 4.8112660000 & 3.6523854000 \\
\hline H48 & 4.2547212000 & 4.5967153000 & 1.9564624000 \\
\hline H49 & 6.0740036000 & $2.03717510 \odot \odot$ & 1. $393986700 \odot$ \\
\hline $\mathrm{H} 50$ & 7.8534167000 & 0.6021286000 & $\odot .5665342000$ \\
\hline H51 & 7.0723805000 & $-\odot .86777200 \odot \odot$ & 1.3768298000 \\
\hline H52 & 1.9466150000 & -2.9170444000 & -5.8133511000 \\
\hline H53 & 3.3854790000 & -1.3097940000 & -6.6564704000 \\
\hline H54 & 2.2698419000 & $2.460166900 \odot$ & -5.3157294000 \\
\hline H55 & 2.8760031000 & $\odot .9809872000$ & -6.9382503000 \\
\hline H56 & $\odot .5728541000$ & $3.404427000 \odot$ & -3.5986674000 \\
\hline
\end{tabular}




\begin{tabular}{|c|c|c|c|}
\hline H57 & $-\odot .6017404000$ & 2.0709094000 & -3.7824122000 \\
\hline H58 & - ๑ . $019 \odot 941 \odot \odot \odot$ & 2.4990763000 & -2.1619143000 \\
\hline H59 & 3.1600478000 & 3. 1017370000 & -2.9506026000 \\
\hline H60 & 2.6817557000 & 2.3045653000 & -1.4269087000 \\
\hline H61 & $3.91648090 \odot \odot$ & $1.55851820 \odot \odot$ & $-2.47035890 \odot \odot$ \\
\hline H62 & -1.1292471000 & -1.0572945000 & -6.6367230000 \\
\hline H63 & $-1.897577500 \odot$ & $-\odot .48651350 \odot \odot$ & $-5.12910 \odot 400 \odot$ \\
\hline H64 & $-\odot .5 \odot 23169 \odot \odot \odot$ & ๑. $381555100 \odot$ & $-5.798252300 \odot$ \\
\hline H65 & $-\odot .795018800 \odot$ & $-3.616782500 \odot$ & -5.8010994000 \\
\hline H66 & 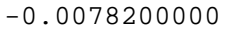 & $-4.063640100 \odot$ & $-4.259684400 \odot$ \\
\hline H67 & -1.5919059000 & -3.2799046000 & -4.2418084000 \\
\hline
\end{tabular}

Energy components, in hartrees:

(A) Nuclear repulsion.......... 4376.09430760965

(E) Total one-electron terms ..... -12067.82214208517

(I) Total two-electron terms..... 5285.17372351572

(J) Coulomb............... 5542.96826290589

(K) Exchange+Correlation...... $\quad-257.79453939017$

(L) Electronic energy........... $-6782.64841856946 \quad$ (E+I)

(N) Total energy............. $-2406.55411095981 \quad(\mathrm{~A}+\mathrm{L})$

SCFE: SCF energy: DFT $\quad-2406.55411095981$ hartrees

HOMO energy: $-\odot .19972$

LUMO energy: -0.07877

Thermodynamic properties calculated assuming an ideal gas

In the table below, the units for temperature

are kelvins, the units for $\mathrm{U}, \mathrm{H}$, and $\mathrm{G}$ are

$\mathrm{kcal} / \mathrm{mol}$ and the units for $\mathrm{CV}$ and $\mathrm{S}$ are $\mathrm{cal} /(\mathrm{mol} \mathrm{K})$

The zero point energy (ZPE): $343.187 \mathrm{kcal} / \mathrm{mol}$

is not included in $\mathrm{U}, \mathrm{H}$, or $\mathrm{G}$ in the table below

\begin{tabular}{|c|c|c|c|c|c|}
\hline$=$ & $\begin{array}{r}5 \mathrm{~K} \\
\mathrm{U}\end{array}$ & CV & $\mathrm{S}$ & $\mathrm{H}$ & G \\
\hline trans. & 0.889 & 2.981 & 44.938 & 1.481 & -11.917 \\
\hline rot. & 0.889 & 2.981 & 37.059 & 0.889 & -10.160 \\
\hline vib. & 22.106 & 135.180 & 148.966 & 22.106 & -22.308 \\
\hline elec. & $\odot .0 \odot \odot$ & $0.00 \odot$ & $\odot .000$ & 0.000 & $\odot .00 \odot$ \\
\hline total & 23.884 & 141.141 & 230.963 & 24.476 & -44.385 \\
\hline
\end{tabular}

Total internal energy, Utot (SCFE + ZPE + U): $\quad-2405.969146$ hartrees Total enthalpy, Htot (Utot + pV): -2405.968202 hartrees Total Gibbs free energy, Gtot (Htot - $T^{\star} S$ ): -2406.077940 hartrees

\section{Single point calculation at the B3LYP/LACV3P**++//B3LYP/LACVP** level}

Energy components, in hartrees:
(A) Nuclear repulsion.......... 4376.09430760965
(E) Total one-electron terms..... -12067.09505749930
(I) Total two-electron terms..... 5284.04446796767
(J) Coulomb............. 5541.89915137477
(K) Exchange+Correlation....... -257.85468340710
(L) Electronic energy.......... $-6783.05058953163 \quad(E+I)$
(N) Total energy............ $-2406.95628192198 \quad(\mathrm{~A}+\mathrm{L})$

SCFE: SCF energy: DFT $\quad-2406.95628192198$ hartrees

HOMO energy: $\quad-0.21048$

LUMO energy: $\quad-\odot .09097$

B3LYP/LACV3P**++//B3LYP/LACVP** Total Gibbs free energy, Gtot (Htot - $\left.\mathrm{T}^{*} \mathrm{~S}\right)$ : -2406.480111 hartrees 


\section{$\mathrm{MG}^{+}$OTf $^{-}$}

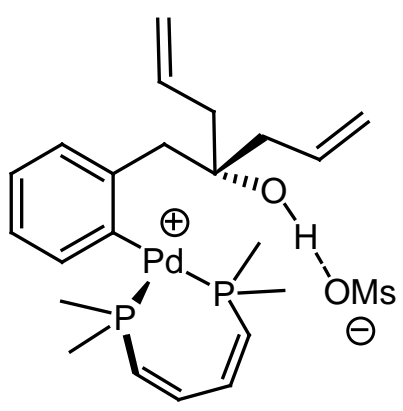

\section{NIMAG $\odot$}

\begin{tabular}{|c|c|c|c|}
\hline C1 & -0.9938712448 & -1.8817942601 & 2.1754411674 \\
\hline $\mathrm{C} 2$ & -1.8864293192 & -2.1467776357 & 1.1383817603 \\
\hline C3 & -1.6561301366 & -1.5822215811 & $-\odot .1212151916$ \\
\hline C4 & $-\odot .5071344861$ & $-\odot .8153547552$ & -0.3303307708 \\
\hline C5 & 0.4346233586 & -0.5771592565 & $\odot .6901951204$ \\
\hline C6 & ๑. 1482532378 & -1.1060048269 & 1.9579952993 \\
\hline 07 & 1. 2793563533 & 3.5473657214 & -0.1625769735 \\
\hline S8 & $\odot .5359663567$ & 3.7355226215 & 1.1283846614 \\
\hline 09 & 1.1641188350 & 3.0087965306 & 2.2700161150 \\
\hline $\mathrm{Pd} 10$ & 0.0449377548 & -0.1832907494 & -2.1605818380 \\
\hline P11 & -1.5966032395 & 1.4060461103 & -2.0891558670 \\
\hline C12 & -0.9498233773 & 3. 0949271137 & -2.3762418918 \\
\hline C13 & 1.6906168262 & ๑. 2202759542 & $\odot .3544079267$ \\
\hline C14 & 2.9001514705 & ๑. 2031100435 & 1.3250561482 \\
\hline 015 & 2.5212808841 & $\odot .6414152540$ & 2.6210466218 \\
\hline C16 & $\odot .7169296624$ & 5.4827584040 & 1.5483761455 \\
\hline 017 & -0.9290079050 & 3.5054998902 & ๑. 9785910317 \\
\hline C18 & 3.4791612377 & -1.2198904327 & 1.5455777767 \\
\hline C19 & 4.0256601750 & -1.9020865554 & ๑. 3231525436 \\
\hline $\mathrm{C} 20$ & 3.5277814191 & -3.0173460760 & -0.2209019791 \\
\hline $\mathrm{C} 21$ & 3.9674542830 & 1.1729213908 & $\odot .7291944054$ \\
\hline $\mathrm{C} 22$ & 5.2008945888 & 1.3063512904 & 1.5746764092 \\
\hline $\mathrm{C} 23$ & 6.4563325050 & 1. 2027285008 & 1.1330346640 \\
\hline P24 & 1.0053004697 & $\odot .5733150500$ & -4.2918103251 \\
\hline $\mathrm{C} 25$ & 1.8090063867 & -0.6731538664 & -5.4018823184 \\
\hline $\mathrm{C} 26$ & -0.0127275186 & 1.5001246529 & -5.4947927332 \\
\hline $\mathrm{C} 27$ & -1.3508250554 & 1.6145851985 & -5.6054333182 \\
\hline $\mathrm{C} 28$ & -2.4654108081 & 1. 0723014171 & -4.8176362900 \\
\hline C29 & -2.6659021615 & ๑. 9530756698 & -3.4897650179 \\
\hline C30 & 2.3817326098 & 1.7688712552 & -3.9768331662 \\
\hline C31 & -2.7459517548 & 1.5834232476 & -0.6790574172 \\
\hline H32 & ๑. 1928497075 & 5.6668261948 & 2.4871584054 \\
\hline H33 & 1.7793107960 & 5.7047255294 & 1.6577932229 \\
\hline H34 & ๑. 2827451276 & 6.0841494768 & ๑. 7479852869 \\
\hline H35 & $\odot .8244573620$ & - ๑. 8815163197 & 2.7748523728 \\
\hline H36 & -1.1873991676 & -2.2754835173 & 3. 1698678382 \\
\hline H37 & -2.7681018842 & -2.7595319844 & 1.3082045541 \\
\hline H38 & -2.3705816792 & -1.7452919109 & -0.9248847041 \\
\hline H39 & 2.0701092603 & $-\odot .1335092140$ & -0.6176933175 \\
\hline $\mathrm{H} 40$ & 1.4151674908 & 1.2685864535 & ๑. 2004671710 \\
\hline $\mathrm{H} 41$ & 2. 0351031348 & 1. 4920497424 & 2.5267533098 \\
\hline $\mathrm{H} 42$ & 4.2406329152 & ๑. 8612710503 & $-\odot .2882916393$ \\
\hline H43 & 3.4774191837 & 2.1520316419 & $\odot .6365521224$ \\
\hline $\mathrm{H} 44$ & 4.2806755924 & -1.1118658554 & 2. 2878035157 \\
\hline H45 & 2.7058066444 & -1.8449733484 & 2.0008046022 \\
\hline $\mathrm{H} 46$ & 5.0139597052 & 1.5115251128 & 2.6283156180 \\
\hline $\mathrm{H} 47$ & 7.3081200643 & 1.3283950474 & 1.7961856584 \\
\hline H48 & 6.6823320672 & $\odot .9994604378$ & ๑. . 0872942971 \\
\hline H49 & 4.9139369394 & -1.4530119215 & -0.1233736344 \\
\hline H50 & 3.9865803433 & -3.4856461456 & -1.0879672521 \\
\hline H51 & 2.6547526713 & -3.5145527354 & $\odot .1972649339$ \\
\hline H52 & ๑.5784902981 & 1.9953914684 & -6.2673429601 \\
\hline H53 & -1.6980256550 & 2.1589990333 & -6.4860441963 \\
\hline H54 & -3.6084337332 & $\odot .5060621502$ & -3.1750100259 \\
\hline H55 & -3.3031352633 & ๑. 7693408840 & -5.4477846897 \\
\hline H56 & -3.5600713671 & 2.2535149228 & $-\odot .9760360967$ \\
\hline H57 & -3.1437946237 & $\odot .6157363227$ & $-\odot .3734520338$ \\
\hline H58 & -2.1831762543 & 2.0446116448 & ๑.1413917999 \\
\hline H59 & -1.7865405329 & 3.7982898379 & -2.3043089665 \\
\hline
\end{tabular}




$\begin{array}{lrrr}\text { H60 } & -0.2073861097 & 3.3229675760 & -1.5994712509 \\ \text { H61 } & -0.4987895646 & 3.1694459076 & -3.3672552112 \\ \text { H62 } & 2.2637433730 & -0.1974369877 & -6.2760456148 \\ \text { H63 } & 2.5841960391 & -1.2017438723 & -4.8402074875 \\ \text { H64 } & 1.0650945654 & -1.3995825073 & -5.7354178290 \\ \text { H65 } & 2.7792824332 & 2.1820070018 & -4.9094044956 \\ \text { H66 } & 2.0302298005 & 2.5791106725 & -3.3339810206 \\ \text { H67 } & 3.1840238984 & 1.2495131893 & -3.4461910240\end{array}$

Energy components, in hartrees:

(A) Nuclear repulsion.......... 4362.30421816321

(E) Total one-electron terms..... -12040.13693637515

(I) Total two-electron terms..... 5271.24749189422

(J) Coulomb.............. 5529.09969636025

(K) Exchange+Correlation....... -257.85220446604

(L) Electronic energy........... $-6768.88944448093 \quad$ (E+I)

(N) Total energy............ $-2406.58522631772 \quad(\mathrm{~A}+\mathrm{L})$

SCFE: SCF energy: DFT $\quad-2406.58522631772$ hartrees

HOMO energy: -0.20543

LUMO energy: $-\odot .11243$

Thermodynamic properties calculated assuming an ideal gas

In the table below, the units for temperature

are kelvins, the units for $\mathrm{U}, \mathrm{H}$, and $\mathrm{G}$ are

$\mathrm{kcal} / \mathrm{mol}$ and the units for $\mathrm{Cv}$ and $\mathrm{S}$ are $\mathrm{cal} /(\mathrm{mol} \mathrm{K})$

The zero point energy (ZPE): $344.252 \mathrm{kcal} / \mathrm{mol}$

is not included in $U, H$, or $G$ in the table below

$\mathrm{T}=298.15 \mathrm{~K}$

\begin{tabular}{rrrrrr} 
& \multicolumn{1}{c}{ U } & \multicolumn{1}{c}{ CV } & \multicolumn{1}{c}{ S } & \multicolumn{1}{c}{ H } & \multicolumn{1}{c}{ G } \\
trans. & 0.889 & 2.981 & 44.938 & 1.481 & -11.917 \\
rot. & 0.889 & 2.981 & 37.047 & 0.889 & -10.157 \\
vib. & 22.434 & 135.925 & 154.629 & 22.434 & -23.669 \\
elec. & 0.000 & 0.000 & 0.000 & 0.000 & 0.000 \\
total & 24.211 & 141.887 & 236.614 & 24.804 & -45.743
\end{tabular}

Total internal energy, Utot (SCFE + ZPE + U): -2405.998033 hartrees Total enthalpy, Htot (Utot + pV): -2405.997089 hartrees

Total Gibbs free energy, Gtot (Htot - $T^{\star} S$ ): -2406.109512 hartrees

\section{Single point calculation at the B3LYP/LACV3P**++//B3LYP/LACVP** level}

Energy components, in hartrees:

(A) Nuclear repulsion......... 4362.30421816102

(E) Total one-electron terms ..... -12039.29883271783

(I) Total two-electron terms.... 5270.00527216651

(J) Coulomb.............. 5527.91200586184

(K) Exchange+Correlation...... $\quad-257.90673369534$

(L) Electronic energy.......... $-6769.29356055132 \quad$ (E+I)

(N) Total energy............ $-2406.98934239030 \quad(\mathrm{~A}+\mathrm{L})$

SCFE: SCF energy: DFT $\quad-2406.98934239030$ hartrees

HOMO energy: -0.22558

LUMO energy: -0.12192

B3LYP/LACV3P**++//B3LYP/LACVP** Total Gibbs free energy, Gtot (Htot - T*S): -2406.513628 hartrees 
$\mathrm{MG}^{+}$<smiles>C=CCC(O)(CC=C)Cc1ccccc1[Pb]1[PH](C)(C)C=CC=CP1(C)(C)C</smiles>

\section{NIMAG $\odot$}

\begin{tabular}{|c|c|c|c|}
\hline C1 & ๑. 2749828611 & -1.4097384402 & 1. 9051569706 \\
\hline $\mathrm{C} 2$ & $-\odot .7515813890$ & -2.3421179590 & 2.0537215461 \\
\hline C3 & -1.4545882827 & -2.8035114390 & ๑.9390813698 \\
\hline C4 & -1.1218163406 & -2.3213233430 & $-\odot .3289082030$ \\
\hline $\mathrm{C} 5$ & -0.0672821385 & -1.4117900343 & -0.4753015122 \\
\hline C6 & $\odot .6367399388$ & -0.9083647724 & $\odot .6416282596$ \\
\hline $\mathrm{Pd} 7$ & $\odot .7589917876$ & -1.0242477317 & -2.2721454557 \\
\hline P8 & 2.0969897632 & -0.7118785714 & -4.3375286870 \\
\hline $\mathrm{Cg}$ & 3.4719337138 & 0.4974757254 & -4.0797240295 \\
\hline C10 & 1.7567060449 & 0.1124165203 & $\odot .4617077361$ \\
\hline C11 & 1.8781575620 & 1.2722873044 & 1.5127766687 \\
\hline C12 & 2. 2597044045 & 2.5729109393 & $\odot .7556479362$ \\
\hline C13 & 2.4594360744 & 3.7710648289 & 1.6441405132 \\
\hline C14 & 3.5224534185 & 4.5765803811 & 1.6065717565 \\
\hline P15 & -1.0630644580 & $\odot .1175417767$ & -3.0134543005 \\
\hline C16 & -2.5637338362 & ๑. 2883871555 & -1.9729651650 \\
\hline C17 & -0.6312197575 & 1.8623774306 & -3.4146639573 \\
\hline C18 & -1.6614281471 & -0.6951226165 & -4.5175238740 \\
\hline C19 & -1.1320697027 & -0.7237479329 & -5.7565353981 \\
\hline $\mathrm{C} 20$ & 0.0771875196 & -0.1046158061 & -6.3177135784 \\
\hline C21 & 1.3430643048 & $-\odot .0326388904$ & -5.8606911623 \\
\hline 022 & $\odot .6607170516$ & 1.4840217682 & 2. 2274194651 \\
\hline $\mathrm{C} 23$ & 2.9129874532 & ๑. 9365496692 & 2.6206035785 \\
\hline C24 & 4.3371344673 & ๑. 8312372687 & 2.1472005654 \\
\hline $\mathrm{C} 25$ & 5.0466306292 & -0.3019155819 & 2.1200323602 \\
\hline $\mathrm{C} 26$ & 2.9748952711 & -2.2202630973 & -4.9473816512 \\
\hline $\mathrm{H} 27$ & $\odot .7882524727$ & -1.0421235935 & 2.7853741530 \\
\hline $\mathrm{H} 28$ & -1.0112725390 & -2.6966619630 & 3. 0466301924 \\
\hline $\mathrm{H} 29$ & -2.2508430995 & -3.5336744894 & 1.0497471022 \\
\hline H3० & -1.6589811709 & -2.6910830391 & -1.1978828634 \\
\hline H31 & 2.7267145713 & -0.4006683750 & ๑. 4290383709 \\
\hline H32 & 1.6269670435 & $\odot .5724447948$ & -0.5308734829 \\
\hline H33 & -0.0453262117 & 1.6697523220 & 1.5936867373 \\
\hline H34 & 3.1532714852 & 2.3976934593 & ๑. 1438914328 \\
\hline H35 & 1.4355526078 & 2.7758689785 & $\odot .0491794230$ \\
\hline H36 & 2.8300934305 & 1.7300450527 & 3.3715408365 \\
\hline H37 & 2.6152522499 & $\odot .0035541052$ & 3.1083179107 \\
\hline H38 & 1.6579270898 & 3.9664256540 & 2.3540924416 \\
\hline H39 & 3.6096582479 & 5.4360898478 & 2.2642199777 \\
\hline $\mathrm{H} 40$ & 4.3436993584 & 4.4100684758 & ๑. 9129641812 \\
\hline H41 & 4.8097973881 & 1.7599280942 & 1.8306198376 \\
\hline $\mathrm{H} 42$ & 6.0821323127 & $-\odot .3206058585$ & 1. 7921647041 \\
\hline $\mathrm{H} 43$ & 4.6251763106 & -1.2475067160 & 2.4541686161 \\
\hline H44 & 2.0629994891 & ๑. 4678506271 & -6.5097806508 \\
\hline H45 & $-\odot .0865117942$ & ๑. 3022771246 & -7.3164626629 \\
\hline H46 & -2.5855248375 & -1.2487907225 & -4.3565899061 \\
\hline H47 & -1.7265598812 & -1.2455949243 & -6.5079739907 \\
\hline H48 & -3.2645365583 & 0.9752942498 & -2.4529095634 \\
\hline H49 & -3.0444170805 & -0.6793116570 & -1.8249470929 \\
\hline $\mathrm{H} 50$ & -2.2816512787 & $\odot .6773077336$ & $-\odot .9948399763$ \\
\hline H51 & -1.4958974200 & 2.3603639711 & -3.8648952364 \\
\hline H52 & -0.3569462581 & 2.3747304510 & -2.4892362159 \\
\hline H53 & ๑. 2090842012 & 1.9078495270 & -4.1078854030 \\
\hline H54 & 3.6056351720 & -1.9934157131 & -5.8121079489 \\
\hline H55 & 3.6004105558 & -2.6167548475 & -4.1422020081 \\
\hline H56 & 2.2427947593 & -2.9830515147 & -5.2259336796 \\
\hline H57 & 4.1084079438 & $\odot .5718968992$ & -4.9670666176 \\
\hline H58 & 3.0631308939 & 1.4839964520 & -3.8472454354 \\
\hline
\end{tabular}


H59 $4.0851642283 \quad 0.1740686004 \quad-3.2338715742$

Energy components, in hartrees:
(A) Nuclear repulsion...........
3010.59991322568
(E) Total one-electron terms.... -8400.00341551117
(I) Total two-electron terms.... 3646.74189788738
(J) Coulomb................ 3846.12564236422
(K) Exchange+Correlation. . . . . $\quad-199.38374447684$
(L) Electronic energy. . . . . . . . $\quad-4753.26151762379$
(N) Total energy............... -1742.66160439810
$(E+I)$
$(A+L)$

SCFE: SCF energy: DFT $\quad-1742.66160439810$ hartrees

HOMO energy: $\quad-0.32590$

LUMO energy: $\quad-0.21877$

Thermodynamic properties calculated assuming an ideal gas

In the table below, the units for temperature are kelvins, the units for $U, H$, and $G$ are $\mathrm{kcal} / \mathrm{mol}$ and the units for $\mathrm{Cv}$ and $\mathrm{S}$ are $\mathrm{cal} /(\mathrm{mol} \mathrm{K})$

The zero point energy (ZPE): $310.747 \mathrm{kcal} / \mathrm{mol}$ is not included in $U, H$, or $G$ in the table below

$\mathrm{T}=298.15 \mathrm{~K}$

\begin{tabular}{|c|c|c|c|c|c|}
\hline & U & CV & $\mathrm{S}$ & $\mathrm{H}$ & G \\
\hline & - - - - - - & $-----\cdot-$ & 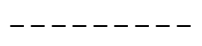 & -------- & ------- \\
\hline trans. & 0.889 & 2.981 & 44.401 & 1.481 & -11.757 \\
\hline rot & $\odot .889$ & 2.981 & 36.291 & 0.889 & -9.931 \\
\hline vib. & 18.152 & 112.687 & 121.545 & 18.152 & -18.087 \\
\hline elec. & 0.000 & $\odot . \odot \odot \odot$ & $\odot .0 \odot \odot$ & 0.000 & $0.00 \odot$ \\
\hline total & 19.929 & 118.649 & 202.236 & 20.522 & -39.775 \\
\hline
\end{tabular}

Total internal energy, Utot (SCFE + ZPE + U): -1742.134639 hartrees Total enthalpy, Htot (Utot + pV): -1742.133695 hartrees Total Gibbs free energy, Gtot (Htot - $T^{*} \mathrm{~S}$ ): -1742.229784 hartrees

\section{Single point calculation at the B3LYP/LACV3P**++//B3LYP/LACVP** level}

Energy components, in hartrees:
(A) Nuclear repulsion. . . . . . . . . 3010.59991322568
(E) Total one-electron terms .... -8399.80595572896
(I) Total two-electron terms.... 3646.26009999655
(J) Coulomb............... 3845.68841185077
(K) Exchange+Correlation...... $\quad-199.42831185422$

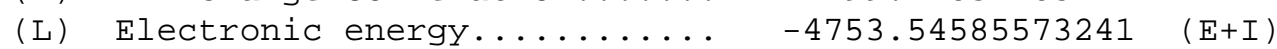
(N) Total energy............. -1742.94594250672 (A+L)

SCFE: SCF energy: DFT $\quad-1742.94594250672$ hartrees

HOMO energy: $\quad-0.33747$

LUMO energy: $\quad-0.22598$

B3LYP/LACV3P**++//B3LYP/LACVP** Total Gibbs free energy, Gtot (Htot - $T^{*} S$ ): -1742.514123 hartrees 


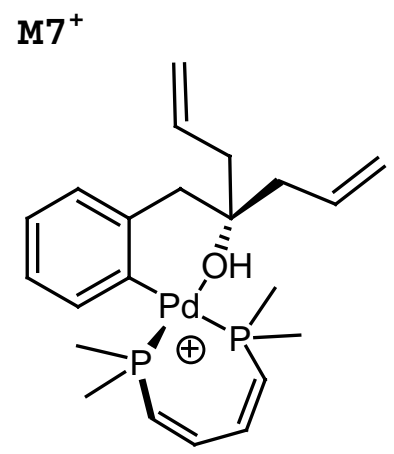

\section{NIMAG $\odot$}

\begin{tabular}{|c|c|c|c|}
\hline C1 & -1.6359290025 & ๑. 9218902052 & -2.2683759691 \\
\hline C2 & -1.4129306928 & ๑. 5114293598 & -0.9480379507 \\
\hline C3 & $-\odot .0972226150$ & ๑. 3179614342 & $-\odot .4859651037$ \\
\hline $\mathrm{C} 4$ & ๑. 9673467327 & ๑. 4916397158 & -1.3848171793 \\
\hline C5 & ๑. 7408263121 & ๑. 8704103660 & -2.7087353804 \\
\hline C6 & -0.5621620155 & 1.1070979471 & -3.1487851015 \\
\hline $\mathrm{Pd} 7$ & -2.9033592070 & ๑. 4243980082 & ๑. 4568501963 \\
\hline P8 & -3.9479451285 & -1.2211929021 & -0.7514793923 \\
\hline C9 & -5.1458928817 & $-\odot .5585176334$ & -1.9878894526 \\
\hline C10 & ๑.1606003915 & $-\odot .0533508718$ & ๑. 9615609375 \\
\hline C11 & $-\odot .2318046958$ & 1.0292180635 & 2. 0130213453 \\
\hline C12 & ๑. 8704984759 & 2.0834707322 & 2.2635248895 \\
\hline C13 & 1.3808570259 & 2.8508898969 & 1.0725242222 \\
\hline C14 & 2.6693579095 & 2.9543371616 & 0.7433592793 \\
\hline 015 & -1.4579822250 & 1.7361208756 & 1.5797038504 \\
\hline C16 & -0.6232529925 & ๑. 3344855517 & 3.3330333460 \\
\hline C17 & -0.8881017993 & 1.2544503023 & 4.4962234737 \\
\hline C18 & $-\odot .3999522397$ & 1.0808819191 & 5.7256718234 \\
\hline P19 & -4.6760210896 & ๑. 4856202289 & 2.1554542816 \\
\hline C20 & -6.4432466514 & ๑.5716721704 & 1.6188116060 \\
\hline $\mathrm{C} 21$ & -4.5860705974 & 1.7954703178 & 3.4477966311 \\
\hline C22 & -4.5251329791 & -1.0495239161 & 3.1200105877 \\
\hline C23 & -4.7088982048 & -2.3125292332 & 2.6889116310 \\
\hline $\mathrm{C} 24$ & -5.1688931467 & -2.8314106884 & 1.3944798370 \\
\hline $\mathrm{C} 25$ & -4.9692222008 & -2.4689270359 & 0.1102588553 \\
\hline C26 & -2.8515094175 & -2.3192100204 & -1.7436831763 \\
\hline $\mathrm{H} 27$ & 1.9839647515 & $\odot .3385839930$ & -1.0339346897 \\
\hline H28 & 1.5791659430 & ๑. 9929543925 & -3.3877148794 \\
\hline $\mathrm{H} 29$ & -0.7492018678 & 1.4276929317 & -4.1697353597 \\
\hline H30 & -2.6444261839 & 1.1109297789 & -2.6263526093 \\
\hline H31 & $-\odot .4079761780$ & $-\odot .9619526213$ & 1.1991394591 \\
\hline H32 & 1.2179067404 & -0.2961470155 & 1.1072996315 \\
\hline H33 & -1.1972288661 & 2.3776340181 & $\odot .9005608951$ \\
\hline H34 & -3.4454263085 & -3.0773078024 & -2.2628733119 \\
\hline H35 & -2.2967276019 & -1.7188950036 & -2.4663871725 \\
\hline H36 & -2.1386635919 & -2.8109504321 & -1.0780324122 \\
\hline H37 & -4.6179550721 & 0.0725571183 & -2.7058252887 \\
\hline H38 & -5.9074998029 & 0.0425773722 & -1.4869080189 \\
\hline H39 & -5.6294649622 & -1.3793979047 & -2.5263278688 \\
\hline $\mathrm{H} 40$ & -5.4044961481 & 1.6901311151 & 4.1661835027 \\
\hline H41 & -3.6332112502 & 1. 7295997700 & 3.9757100697 \\
\hline $\mathrm{H} 42$ & -4.6502561953 & 2.7783196760 & 2.9735673507 \\
\hline H43 & -7.1067728056 & $\odot .5406612680$ & 2.4872775861 \\
\hline H44 & -6.6036774802 & 1.5088266452 & 1.0779238669 \\
\hline H45 & -6.6853388557 & -0.2641737842 & 0.9603107265 \\
\hline H46 & -4.2040734088 & -0.9151204729 & 4.1525037614 \\
\hline $\mathrm{H} 47$ & -4.5792340054 & -3.0987479350 & 3.4329581843 \\
\hline H48 & -5.4493291906 & -3.1112781588 & -0.6311483930 \\
\hline H49 & -5.7708416871 & -3.7341970126 & 1.5133948712 \\
\hline H50 & $\odot .4763955174$ & 2.7888287835 & 3. 0066173732 \\
\hline H51 & 1.7079489944 & 1.5688039845 & 2.7483223689 \\
\hline H52 & -1.5163085761 & $-\odot .2708813970$ & 3.1159368836 \\
\hline H53 & ๑.1708123870 & -0.3753196454 & 3.5929972810 \\
\hline H54 & -1.5292605270 & 2.1118735552 & 4.2955923350 \\
\hline H55 & -0.6380657165 & 1.7650253769 & 6.5346094907 \\
\hline H56 & ๑. 2555261618 & ๑. 2474819790 & 5.9687672869 \\
\hline H57 & $\odot .6525296359$ & 3. 4058447595 & 0.4770554083 \\
\hline H58 & 2.9954731331 & 3.5573829179 & -0.0982165550 \\
\hline H59 & 3. 4448799552 & 2.4473157385 & 1.3132003246 \\
\hline
\end{tabular}


Energy components, in hartrees:

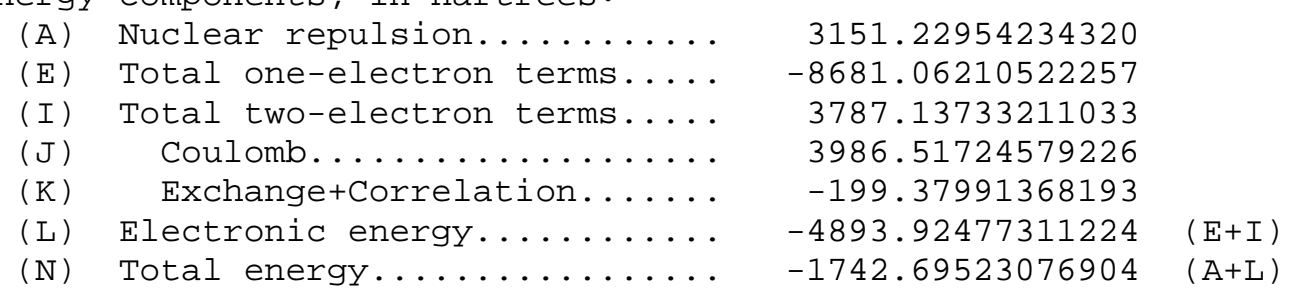

SCFE: SCF energy: DFT $\quad-1742.69523076904$ hartrees

HOMO energy: $-\odot .32834$

LUMO energy: $\quad-\odot .18114$

Thermodynamic properties calculated assuming an ideal gas

In the table below, the units for temperature

are kelvins, the units for $\mathrm{U}, \mathrm{H}$, and $\mathrm{G}$ are

$\mathrm{kcal} / \mathrm{mol}$ and the units for $\mathrm{Cv}$ and $\mathrm{S}$ are $\mathrm{cal} /(\mathrm{mol} \mathrm{K})$

The zero point energy (ZPE): $\quad 312.188 \mathrm{kcal} / \mathrm{mol}$

is not included in $U, H$, or $G$ in the table below

$\mathrm{T}=298.15 \mathrm{~K}$

\begin{tabular}{rrrrrr} 
& \multicolumn{1}{c}{ U } & \multicolumn{1}{c}{ CV } & \multicolumn{1}{c}{ S } & \multicolumn{1}{c}{ H } & \multicolumn{1}{c}{ G } \\
trans. & 0.889 & 2.981 & 44.401 & 1.481 & -11.757 \\
rot. & 0.889 & 2.981 & 35.827 & 0.889 & -9.793 \\
vib. & 18.025 & 113.303 & 117.604 & 18.025 & -17.039 \\
elec. & 0.000 & 0.000 & 0.000 & 0.000 & 0.000 \\
total & 19.803 & 119.264 & 197.832 & 20.395 & -38.589
\end{tabular}

Total internal energy, Utot (SCFE + ZPE + U): $\quad-1742.166170$ hartrees Total enthalpy, Htot (Utot + pV): -1742.165226 hartrees Total Gibbs free energy, Gtot (Htot - $T^{\star} S$ ): $\quad-1742.259222$ hartrees

\section{Single point calculation at the B3LYP/LACV3P**++//B3LYP/LACVP** level}

Energy components, in hartrees:
(A) Nuclear repulsion.......... 3151.22954234320
(E) Total one-electron terms.... -8681.01800425622
(I) Total two-electron terms.... 3786.81182032443
(J) Coulomb............ 3986.25333387315
(K) Exchange+Correlation....... -199.44151354872
(L) Electronic energy......... -4894.20618393179
(N) Total energy............ $-1742.97664158860 \quad(\mathrm{~A}+\mathrm{L})$

SCFE: SCF energy: DFT $\quad-1742.97664158860$ hartrees

HOMO energy: $\quad-0.33712$

LUMO energy: $\quad-0.19017$

B3LYP/LACV3P**++//B3LYP/LACVP** Total Gibbs free energy, Gtot (Htot - $\left.\mathrm{T}^{*} \mathrm{~S}\right)$ : -1742.540633 hartrees 


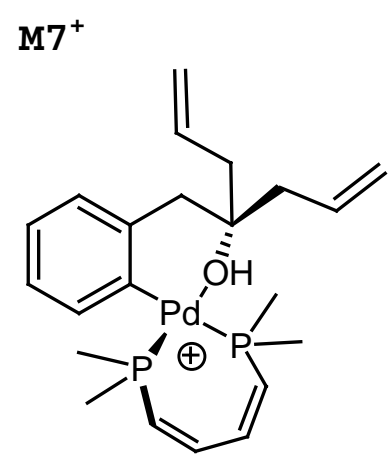

more stable conformer

\section{NIMAG $\odot$}

\begin{tabular}{|c|c|c|c|}
\hline C1 & -0.1677369304 & ๑. 2036555807 & $-\odot .0841791886$ \\
\hline C2 & 1.0852210285 & $\odot .3436375748$ & -0.7021092671 \\
\hline C3 & 1. 2612617013 & 1.2058905810 & -1.7868525104 \\
\hline C4 & ๑.1815301344 & 1.9557267823 & -2.2553128051 \\
\hline C5 & -1.0792393688 & 1.8036061577 & -1.6631350955 \\
\hline C6 & -1.2653830293 & ๑. 9185487941 & - ๑. 5951919379 \\
\hline $\mathrm{Pd} 7$ & -3.0143423439 & ๑. 7496006395 & ๑. 4592576958 \\
\hline P8 & -5.0462076928 & ๑. 6109929851 & 1.8387621727 \\
\hline C9 & -5.4080455456 & -1.1634770853 & 2.0144396986 \\
\hline C10 & -5.7800725649 & -2.0235036748 & 1.0475192647 \\
\hline C11 & -6.0926700423 & -1.7961003046 & -0.3689768511 \\
\hline C12 & -5.5609976983 & -1.0129823406 & -1.3299478427 \\
\hline P13 & -4.1163466408 & ๑. 1019587193 & -1.4458493841 \\
\hline C14 & -4.8374387425 & 1.5320571821 & -2.3613368810 \\
\hline C15 & -0.3266036394 & $-\odot .6471915022$ & 1.1561099077 \\
\hline C16 & -0.5999319491 & $\odot .1542040803$ & 2.4587288851 \\
\hline C17 & -1.0972145985 & $-\odot .8208351818$ & 3.5498475850 \\
\hline C18 & -1.3953477991 & $-\odot .1885002548$ & 4.8839768239 \\
\hline C19 & $-\odot .9661027171$ & $-\odot .6519086264$ & 6.0589894511 \\
\hline C20 & ๑. 4912370050 & 3.4652877449 & 2.7622202873 \\
\hline $\mathrm{C} 21$ & $\odot .8446163776$ & 2.2827305781 & 2.2420757373 \\
\hline $\mathrm{C} 22$ & 0.6306289644 & 0.9709768715 & 2.9568607547 \\
\hline $\mathrm{C} 23$ & -3.1198553489 & $-\odot .7886143258$ & -2.7128684031 \\
\hline 024 & -1.7070364011 & 1.0988939968 & 2.2336357955 \\
\hline C25 & -6.6267555825 & 1.3936661513 & 1.2836269409 \\
\hline C26 & -4.9114510716 & 1.1749850285 & 3.5870796988 \\
\hline $\mathrm{H} 27$ & 1.2839251411 & 2.2489608060 & 1. 2476877094 \\
\hline H28 & 1.9307164858 & -0.2255526170 & -0.3217962185 \\
\hline H29 & 2.2368604849 & 1.2982302074 & -2.2541695288 \\
\hline H30 & ๑. 3105769139 & 2.6495502619 & -3.0812740897 \\
\hline H31 & -1.9083326568 & 2.3938295770 & -2.0438151543 \\
\hline H32 & -1.1653907954 & -1.3424983934 & 1.0182723904 \\
\hline H33 & $\odot .5666172541$ & -1.2592862069 & 1.3199460918 \\
\hline H34 & -3.7472892436 & -1.0533377080 & -3.5690332471 \\
\hline H35 & -2.2924485556 & -0.1556262873 & -3.0369642172 \\
\hline H36 & -2.7099547406 & -1.6984148876 & -2.2686442810 \\
\hline H37 & -4.0402164441 & 2.2146272000 & -2.6628715719 \\
\hline H38 & -5.5341296000 & 2.0729075985 & -1.7168147743 \\
\hline H39 & -5.3657307887 & 1.1862502636 & -3.2549620872 \\
\hline $\mathrm{H} 40$ & -5.8357810960 & ๑. 9710504850 & 4.1358763665 \\
\hline H41 & -4.0788333557 & ๑. 6668589956 & 4.0766959788 \\
\hline $\mathrm{H} 42$ & -4.7154859420 & 2.2501569430 & 3.6058612962 \\
\hline $\mathrm{H} 43$ & -7.4311697201 & 1.1742405406 & 1.9913393095 \\
\hline H44 & -6.4839423693 & 2.4768084335 & 1. 2264490232 \\
\hline H45 & -6.9119643469 & 1.0211944368 & ๑. 2982048445 \\
\hline H46 & -5.2644292113 & -1.5670480758 & 3. 0161962682 \\
\hline $\mathrm{H} 47$ & -5.9675617134 & -3.0509791984 & 1.3599298748 \\
\hline H48 & -6.0223620530 & -1.1087048887 & -2.3150589241 \\
\hline H49 & -6.9052316392 & -2.4385145433 & -0.7131693888 \\
\hline H50 & $\odot .4918356506$ & 1.1691966514 & 4.0241655382 \\
\hline H51 & 1.5150150619 & ๑. 3308846849 & 2.8630502453 \\
\hline H52 & -2.0048781345 & -1.3005124754 & 3.1546609779 \\
\hline H53 & $-\odot .3532879541$ & -1.6172437222 & 3.6698420209 \\
\hline H54 & -2.0154740321 & $\odot .7072511180$ & 4.8602497669 \\
\hline H55 & -1.2330974266 & -0.1662052087 & 6.9928830181 \\
\hline H56 & -0.3372921501 & -1.5367598955 & 6.1315355666 \\
\hline H57 & -1.2978191284 & 1.9786742739 & 2.1063123502 \\
\hline H58 & $\odot .6492471554$ & 4.3928536309 & 2.2211757885 \\
\hline H59 & ๑.0689807930 & 3.5476925139 & 3.7619234636 \\
\hline
\end{tabular}


Energy components, in hartrees:

(A) Nuclear repulsion........... 3157.15875672951

(E) Total one-electron terms..... -8693.02191181557

(I) Total two-electron terms..... 3793.16072637582

(J) Coulomb................ 3992.54256357035

(K) Exchange+Correlation . . . . . . $\quad-199.38183719453$

(L) Electronic energy........... -4899.86118543975

(N) Total energy............ $-1742.70242871024 \quad(\mathrm{~A}+\mathrm{L})$

SCFE: SCF energy: DFT $\quad-1742.70242871024$ hartrees

HOMO energy: $\quad-0.32862$

LUMO energy: -0.17925

Thermodynamic properties calculated assuming an ideal gas

In the table below, the units for temperature

are kelvins, the units for $U, H$, and $G$ are

$\mathrm{kcal} / \mathrm{mol}$ and the units for $\mathrm{Cv}$ and $\mathrm{S}$ are $\mathrm{cal} /(\mathrm{mol} \mathrm{K})$

The zero point energy (ZPE): $312.432 \mathrm{kcal} / \mathrm{mol}$

is not included in $U, H$, or $G$ in the table below

$\mathrm{T}=298.15 \mathrm{~K}$

\begin{tabular}{rrrrrr} 
& \multicolumn{1}{c}{ U } & \multicolumn{1}{c}{ CV } & \multicolumn{1}{c}{ S } & \multicolumn{1}{c}{ H } & \multicolumn{1}{c}{ G } \\
trans. & 0.889 & 2.981 & 44.401 & 1.481 & -11.757 \\
rot. & 0.889 & 2.981 & 35.796 & 0.889 & -9.784 \\
vib. & 17.911 & 112.814 & 116.970 & 17.911 & -16.963 \\
elec. & $0.00 \odot$ & $0.00 \odot$ & 0.000 & 0.000 & 0.000 \\
total & 19.689 & 118.776 & 197.167 & 20.281 & -38.504
\end{tabular}

Total internal energy, Utot (SCFE + ZPE + U): -1742.173161 hartrees Total enthalpy, Htot (Utot + pV): -1742.172217 hartrees Total Gibbs free energy, Gtot (Htot - $\mathrm{T}^{\star} \mathrm{S}$ ): $\quad-1742.265897$ hartrees

\section{Single point calculation at the B3LYP/LACV3P**++//B3LYP/LACVP** level}

Energy components, in hartrees:
(A) Nuclear repulsion........... 3157.15875672951
(E) Total one-electron terms.... -8692.98278284109
(I) Total two-electron terms.... 3792.84121270640
(J) Coulomb............... 3992.28656405275
(K) Exchange+Correlation...... $\quad-199.44535134635$
(L) Electronic energy.......... -4900.14157013469 (E+I)
(N) Total energy............ -1742.98281340518 (A+L)

SCFE: SCF energy: DFT $\quad-1742.98281340518$ hartrees

HOMO energy: $\quad-0.33683$

LUMO energy: -0.18851

B3LYP/LACV3P**++//B3LYP/LACVP** Total Gibbs free energy, Gtot (Htot - $T^{*} S$ ): -1742.546281 hartrees 


\section{$\mathrm{MB}^{+}$}

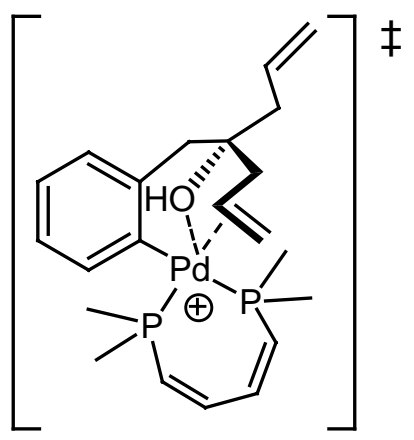

\section{NIMAG 1}

\begin{tabular}{|c|c|c|c|}
\hline C1 & -1.5121040950 & $\odot .5070793487$ & $-\odot .8489024650$ \\
\hline $\mathrm{C} 2$ & $-\odot .2200524032$ & ๑.1798428689 & $-\odot .4026251513$ \\
\hline C3 & ๑. 8185119355 & 0.1027983583 & -1.3482778021 \\
\hline $\mathrm{C} 4$ & $\odot .5988217050$ & $\odot .3566702673$ & -2.7026230366 \\
\hline C5 & -0.6768158720 & $\odot .7221895944$ & -3.1313323917 \\
\hline C6 & -1.7220275660 & ๑. 7999399988 & -2.2019769552 \\
\hline C7 & ๑.1283523392 & -0.0423572732 & 1.0589310862 \\
\hline $\mathrm{C} 8$ & -0.3143743720 & 1.0346986350 & 2. 0822107069 \\
\hline 09 & -1.7258210000 & $\odot .8591360000$ & 2.3818090000 \\
\hline $\operatorname{Pd} 10$ & -3.0544430000 & $\odot .800711000 \odot$ & $\odot .493040000 \odot$ \\
\hline P11 & -4.1108546268 & -0.9857834486 & $-\odot .5427856020$ \\
\hline C12 & -3.0512730560 & -2.2624378740 & -1.3514390205 \\
\hline P13 & -4.9127075298 & 1.0980627650 & 2.0737034701 \\
\hline C14 & -4.8718561047 & 2.5163555720 & 3.2527642682 \\
\hline C15 & -4.7679909301 & -0.3152795284 & 3.2150858784 \\
\hline C16 & -4.9691660392 & -1.6216032060 & 2.9539822743 \\
\hline C17 & -5.4418058209 & -2.2912129147 & 1.7359659186 \\
\hline C18 & -5.2109554139 & -2.0919074628 & $\odot .4232735245$ \\
\hline C19 & -6.6691043587 & 1.1045699674 & 1.4984429381 \\
\hline $\mathrm{C} 20$ & -5.2521103782 & $-\odot .4763597870$ & -1.9032953908 \\
\hline $\mathrm{C} 21$ & 0.5022980695 & $\odot .8407544036$ & 3.3881628708 \\
\hline $\mathrm{C} 22$ & ๑. 0359534336 & 1.6565535143 & 4.5639229669 \\
\hline C23 & ๑. 7925972869 & 2.5407807056 & 5.2163681052 \\
\hline C24 & -0.1883578239 & 2.5010535838 & 1.5883001797 \\
\hline $\mathrm{C} 25$ & -1.1941656913 & 2.9413706077 & 0.5488302103 \\
\hline $\mathrm{C} 26$ & -2.4457194296 & 3. 3683735974 & ๑. 8258374235 \\
\hline $\mathrm{H} 27$ & -0.8622336211 & 2.9560302720 & $-\odot .4832208970$ \\
\hline $\mathrm{H} 28$ & 1.8202016771 & $-\odot .1518736440$ & -1.0084022223 \\
\hline H29 & 1.4197301200 & ๑. 2858215716 & -3.4095877568 \\
\hline H30 & $-\odot .8649641840$ & ๑. 9491352679 & -4.1771457780 \\
\hline H31 & -2.7047852712 & 1.1092452684 & -2.5476733878 \\
\hline H32 & $-\odot .2869964451$ & -1.0001876452 & 1.4092650476 \\
\hline H33 & 1.2155959294 & -0.1495729958 & 1.1373333082 \\
\hline H34 & -3.6755140530 & -3.0602035684 & -1.7649183699 \\
\hline H35 & -2.4648958548 & -1.8007693013 & -2.1465602166 \\
\hline H36 & -2.3663042590 & -2.6857534811 & -0.6133025098 \\
\hline H37 & -4.6795147406 & ๑. 0324070208 & -2.6815130815 \\
\hline H38 & -6.0082333460 & $\odot .2123755587$ & -1.5204899620 \\
\hline H39 & -5.7454284861 & -1.3496690694 & -2.3413136792 \\
\hline $\mathrm{H} 40$ & -5.6397780240 & 2.4030245671 & 4.0236427024 \\
\hline H41 & -3.8882621352 & 2.5658482004 & 3.7266622617 \\
\hline H42 & -5.0480336771 & 3. 4511528224 & 2.7146088904 \\
\hline H43 & -7.3535513443 & 1.1560242902 & 2.3494757751 \\
\hline H44 & -6.8254656296 & 1.9769388426 & 0.8572531638 \\
\hline H45 & -6.8859894904 & ๑. 2019010970 & ๑. 9248832245 \\
\hline H46 & -4.4512284935 & $-\odot .0523038050$ & 4.2245001648 \\
\hline $\mathrm{H} 47$ & -4.8504877091 & -2.3065165037 & 3.7944253119 \\
\hline H48 & -5.7038699177 & -2.7995742785 & $-\odot .2467311290$ \\
\hline H49 & -6.0763649374 & -3.1501413058 & 1.9616700285 \\
\hline H50 & -0.2828149277 & 3.1401784392 & 2.4720776051 \\
\hline H51 & ๑. 8252369625 & 2.6327592849 & 1. 1963195162 \\
\hline H52 & $\odot .4621898455$ & -0.2300520914 & 3.6439572624 \\
\hline H53 & 1.5534712926 & 1.0643550146 & 3.1727886592 \\
\hline H54 & $-\odot .9871838180$ & 1.4844237740 & 4.8954978850 \\
\hline H55 & ๑ . 4160910141 & 3. 0897996439 & 6.0742418852 \\
\hline H56 & 1.8215505484 & 2.7381535843 & 4.9241393525 \\
\hline H57 & -1.8404018543 & $-\odot .0227540546$ & 2.7646586042 \\
\hline H58 & -3.0899149693 & 3. 7564618091 & $\odot .0419504775$ \\
\hline H59 & -2.7867353429 & 3. 4707948874 & 1.8494555828 \\
\hline
\end{tabular}


Energy components, in hartrees:
(A) Nuclear repulsion...........
3219.23213919147
(E) Total one-electron terms.....
$-8817.24691977536$
(I) Total two-electron terms.....
3855.33035921364

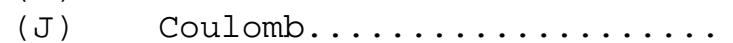
4054.73171535105
(K) Exchange+Correlation...... $\quad-199.40135613741$
(L) Electronic energy........... -4961.91656056172
(N) Total energy............ -1742.68442137025 (A+L)

SCFE: SCF energy: DFT $\quad-1742.68442137025$ hartrees

HOMO energy: $\quad-0.32207$

LUMO energy: -0.17883

Thermodynamic properties calculated assuming an ideal gas

In the table below, the units for temperature

are kelvins, the units for $U, H$, and $G$ are

$\mathrm{kcal} / \mathrm{mol}$ and the units for $\mathrm{Cv}$ and $\mathrm{S}$ are $\mathrm{cal} /(\mathrm{mol} \mathrm{K})$

The zero point energy (ZPE): $312.602 \mathrm{kcal} / \mathrm{mol}$

is not included in $U, H$, or $G$ in the table below

$\mathrm{T}=298.15 \mathrm{~K}$

\begin{tabular}{rrrrrr} 
& \multicolumn{1}{c}{ U } & \multicolumn{1}{c}{ CV } & \multicolumn{1}{c}{ S } & \multicolumn{1}{c}{ H } & \multicolumn{1}{c}{ G } \\
trans. & 0.889 & 2.981 & 44.401 & 1.481 & -11.757 \\
rot. & 0.889 & 2.981 & 35.646 & 0.889 & -9.739 \\
vib. & 17.263 & 110.959 & 109.307 & 17.263 & -15.327 \\
elec. & 0.000 & 0.000 & 0.000 & 0.000 & 0.000 \\
total & 19.040 & 116.920 & 189.354 & 19.633 & -36.823
\end{tabular}

Total internal energy, Utot (SCFE + ZPE + U): -1742.155915 hartrees Total enthalpy, Htot (Utot + pV): -1742.154971 hartrees Total Gibbs free energy, Gtot (Htot - $\mathrm{T}^{\star} \mathrm{S}$ ): -1742.244939 hartrees

\section{Single point calculation at the B3LYP/LACV3P**++//B3LYP/LACVP** level}

Energy components, in hartrees:

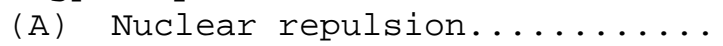
3219.23213919147
(E) Total one-electron terms.....
$-8817.15130816912$
(I) Total two-electron terms.....
3854.95488810320
(J) Coulomb.............. 4054.42027596617
(K) Exchange+Correlation...... $\quad-199.46538786298$
(L) Electronic energy.......... -4962.19642006593 (E+I)
(N) Total energy............ $-1742.96428087446 \quad(\mathrm{~A}+\mathrm{L})$

SCFE: SCF energy: DFT $\quad-1742.96428087446$ hartrees

HOMO energy: $\quad-0.33085$

LUMO energy: $\quad-0.18873$

B3LYP/LACV3P**++//B3LYP/LACVP** Total Gibbs free energy, Gtot (Htot - $\mathrm{T}^{*} \mathrm{~S}$ ): -1742.524799 hartrees 


\section{$\mathrm{M9}^{+}$}

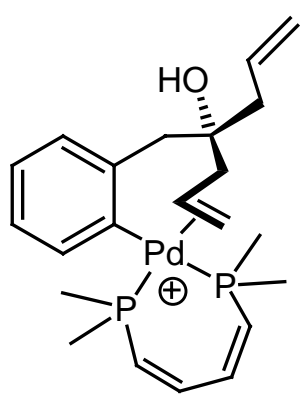

\section{NIMAG $\odot$}

\begin{tabular}{|c|c|c|c|}
\hline C1 & 1.0213584723 & $-\odot .1930276982$ & - 2.1730198918 \\
\hline C2 & -0.0660703919 & 0.4512791974 & -2.7626089003 \\
\hline C3 & -1.2142714555 & ๑. 7156958036 & -2.0038512137 \\
\hline $\mathrm{C} 4$ & -1.2695424991 & ๑. 3408135813 & $-\odot .6596379445$ \\
\hline C5 & -0.1543587244 & -0.2472401812 & $-\odot .0398270378$ \\
\hline C6 & ๑. 9781048563 & $-\odot .5282905979$ & -0.8179546614 \\
\hline $\mathrm{Pd} 7$ & -3.0370400540 & ๑.5143815596 & ๑. 4493311071 \\
\hline C8 & -2.5778339784 & 2. 3920917631 & 1.7936872923 \\
\hline C9 & -1.4486237831 & 2.4690743283 & 1.0251443661 \\
\hline C10 & - ๑. . 0369665956 & 2.1534261674 & 1. 4508957752 \\
\hline C11 & $\odot .2871479673$ & ๑. 8615808543 & 2.2294638309 \\
\hline C12 & 1.8168873041 & ๑. 8080589050 & 2.4934366505 \\
\hline C13 & 2.3817183407 & 2.0135973235 & 3.1939564295 \\
\hline C14 & 3. 3829280988 & 2.7613025448 & 2.7264599487 \\
\hline C15 & $-\odot .1500926572$ & $-\odot .4321873669$ & 1. 4602389181 \\
\hline P16 & -5.1739868786 & $\odot .4574410180$ & 1.6570330247 \\
\hline C17 & -5.6442011969 & 1.7892126755 & 2.8462513634 \\
\hline P18 & -3.6252469349 & -1.4354822307 & $-\odot .7579865361$ \\
\hline C19 & -4.3813580384 & $-\odot .9964240449$ & -2.3839392264 \\
\hline $\mathrm{C} 20$ & -5.1178693706 & $-\odot .9986643949$ & 2.7454059102 \\
\hline C21 & -5.1634899374 & -2.2999400590 & 2.4075638168 \\
\hline $\mathrm{C} 22$ & -5.3328004208 & -2.9358855752 & 1.0980317926 \\
\hline C23 & -4.8439132325 & -2.6475771447 & -0.1230778776 \\
\hline C24 & -6.7195191788 & ๑. 3063869970 & ๑. 6522595982 \\
\hline C25 & -2.2781555884 & -2.6071908264 & -1.2285973832 \\
\hline 026 & $-\odot .4226226818$ & ๑. 9516278638 & 3.4737763033 \\
\hline $\mathrm{H} 27$ & -1.5214627841 & 3.0266145414 & ๑. . 0932524396 \\
\hline H28 & 1.8381232974 & -1.0072622062 & -0.3544812188 \\
\hline H29 & 1.9060320436 & -0.4203288574 & -2.7604127358 \\
\hline H30 & -0.0310999449 & $\odot .7408781863$ & -3.8094399317 \\
\hline H31 & -2.0590574516 & 1.2125193245 & -2.4755937759 \\
\hline H32 & -1.1595043586 & $-\odot .6848620350$ & 1.8032521038 \\
\hline H33 & $\odot .5082617927$ & -1.2586961176 & 1.7540618725 \\
\hline H34 & -2.7097751299 & -3.4641813977 & -1.7543421440 \\
\hline H35 & -1.5545369933 & -2.1070411759 & -1.8731904745 \\
\hline H36 & -1.7689007633 & -2.9594061759 & $-\odot .3293088369$ \\
\hline H37 & -3.6528396704 & $-\odot .4402523474$ & -2.9768908243 \\
\hline H38 & -5.2663762644 & $-\odot .3733180936$ & -2.2366786035 \\
\hline H39 & -4.6653283875 & -1.9016648987 & -2.9294261801 \\
\hline $\mathrm{H} 40$ & -6.5725351134 & 1.5240995498 & 3.3605337512 \\
\hline H41 & -4.8569525289 & 1.9385872589 & 3.5884030517 \\
\hline $\mathrm{H} 42$ & -5.7982080467 & 2.7293861431 & 2.3098305051 \\
\hline H43 & -7.5878437011 & ๑. 1990143824 & 1.3079359504 \\
\hline H44 & -6.8352765032 & 1.2119620541 & ๑. 0497497736 \\
\hline H45 & -6.6720947882 & $-\odot .5570221356$ & -0.0120071507 \\
\hline H46 & -4.9998354432 & $-\odot .7597999453$ & 3.8017935314 \\
\hline $\mathrm{H} 47$ & -5.1427924473 & -3.0153148250 & 3.2302536482 \\
\hline H48 & -5.1261042745 & -3.3407792009 & - ๑. 9180187491 \\
\hline H49 & -5.9378192998 & -3.8428707123 & 1.1493707975 \\
\hline H50 & ๑. 2760421981 & 2.9949454662 & 2.0841474999 \\
\hline H51 & $\odot .6061056956$ & 2.1871554633 & $\odot .5692618674$ \\
\hline H52 & $2.0 \odot 11985610$ & $-\odot .0922390 \odot 61$ & 3.1015406171 \\
\hline H53 & 2.3378057500 & $\odot .6469073018$ & 1.5421269348 \\
\hline H54 & 1.9232950798 & 2.2652918082 & 4.1496211371 \\
\hline H55 & $3.7630 \odot \odot 5244$ & 3.6150730481 & 3. 2795441260 \\
\hline H56 & 3.8693819727 & 2.5419591097 & 1.7782919467 \\
\hline H57 & -0.1483843750 & 0. 2101442359 & 4.0313519884 \\
\hline H58 & -3.4451438841 & 2.9833145621 & 1.5183541565 \\
\hline H59 & -2.5213479136 & 1.9951619283 & 2.8018688313 \\
\hline
\end{tabular}


Energy components, in hartrees:
(A) Nuclear repulsion...........
3218.95527290339
(E) Total one-electron terms.....
$-8816.70957610810$
(I) Total two-electron terms..... 3855.06774671156
(J) Coulomb............... 4054.46726942402
(K) Exchange+Correlation . . . . . . $\quad$-199.39952271246
(L) Electronic energy. . . . . . . . . . -4961.64182939654
(N) Total energy.............. -1742.68655649315
$(E+I)$
$(A+L)$

SCFE: SCF energy: DFT $\quad-1742.68655649315$ hartrees

HOMO energy: $\quad-0.32503$

LUMO energy: $\quad-0.17967$

Thermodynamic properties calculated assuming an ideal gas

In the table below, the units for temperature

are kelvins, the units for $U, H$, and $G$ are

$\mathrm{kcal} / \mathrm{mol}$ and the units for $\mathrm{Cv}$ and $\mathrm{S}$ are $\mathrm{cal} /(\mathrm{mol} \mathrm{K})$

The zero point energy (ZPE): $312.583 \mathrm{kcal} / \mathrm{mol}$

is not included in $U, H$, or $G$ in the table below

$\mathrm{T}=298.15 \mathrm{~K}$

\begin{tabular}{|c|c|c|c|c|c|}
\hline & U & CV & S & $\mathrm{H}$ & G \\
\hline & -------- & $-----\cdot--$ & -------- & -------- & -------- \\
\hline trans. & 0.889 & 2.981 & 44.401 & 1.481 & -11.757 \\
\hline rot. & $\odot .889$ & 2.981 & 35.822 & $\odot .889$ & -9.792 \\
\hline vib. & 17.770 & 113.087 & 114.080 & 17.770 & -16.243 \\
\hline elec. & 0.000 & 0.000 & 0.000 & 0.000 & 0.000 \\
\hline total & 19.547 & 119.049 & 194.303 & 20.140 & -37.791 \\
\hline
\end{tabular}

Total internal energy, Utot (SCFE + ZPE + U): -1742.158412 hartrees Total enthalpy, Htot (Utot + pV): -1742.157468 hartrees Total Gibbs free energy, Gtot (Htot - $\mathrm{T}^{\star} \mathrm{S}$ ): $\quad-1742.249788$ hartrees

\section{Single point calculation at the B3LYP/LACV3P**++//B3LYP/LACVP** level}

Energy components, in hartrees:
(A) Nuclear repulsion.......... 3162.79302816308
(E) Total one-electron terms.... -8704.46503520727
(I) Total two-electron terms..... 3798.7013053888
(J) Coulomb.............. 3998.15811241920
(K) Exchange+Correlation . . . . . $\quad-199.45680703032$
(L) Electronic energy.......... -4905.76372981839 (E+I)
(N) Total energy............. -1742.97070165531 (A+L)

SCFE: SCF energy: DFT $\quad-1742.97070165531$ hartrees

$\begin{array}{ll}\text { HOMO energy: } & -0.33517 \\ \text { LUMO energy: } & -0.18916\end{array}$

B3LYP/LACV3P**++//B3LYP/LACVP** Total Gibbs free energy, Gtot (Htot - $\left.T^{*} \mathrm{~S}\right)$ : -1742.533934 hartrees 
<smiles>C=CC[C@]1(O)Cc2ccccc2[P+]2(c3ccccc3)[PH](C)(C)C=CC=CP2(C)(C)C1</smiles>

NIMAG $\odot$

\begin{tabular}{|c|c|c|c|}
\hline C1 & -0.3694814404 & -0.3032103090 & 2. 3467134388 \\
\hline $\mathrm{C} 2$ & -1.3882091277 & -1.2499544739 & 2. 4689358099 \\
\hline C3 & -1.9013880423 & -1.8714031579 & 1.3286269107 \\
\hline $\mathrm{C} 4$ & -1.4007350717 & -1.5293296255 & 0.0650858520 \\
\hline C5 & -0.3841595019 & $-\odot .5762694239$ & -0.0522457105 \\
\hline C6 & 0.1479766631 & 0.0474404978 & 1.0882970304 \\
\hline $\mathrm{Pd} 7$ & 0.4396806118 & $-\odot .1573190541$ & -1.8845831976 \\
\hline P8 & 1.4724543999 & $\odot .4996992053$ & -4.0602340668 \\
\hline c9 & 2.7131389869 & 1.8504144864 & -3.8063039350 \\
\hline C10 & 1.3266442858 & ๑.9912255930 & 0.9745048465 \\
\hline C11 & 2.7104087729 & ๑.2961170451 & 1.1409561356 \\
\hline $\mathrm{C} 12$ & 3.8301565981 & 1.2484125672 & 0.6439154032 \\
\hline C13 & 5.2216833127 & $\odot .6950829829$ & 0.7963032434 \\
\hline C14 & 6.1324556995 & 0.6544842752 & -0.1778320101 \\
\hline P15 & -1.3642517549 & 1.2750178442 & -2.2623237846 \\
\hline C16 & -2.6639316978 & 1.4740685142 & -0.9831466941 \\
\hline C17 & -0.9097902756 & 3.0182985925 & -2.6547951672 \\
\hline C18 & -2.2776864741 & $\odot .6025428798$ & -3.6813388453 \\
\hline C19 & -1.9604419539 & $\odot .5946327127$ & -4.9909183091 \\
\hline $\mathrm{C} 20$ & -0.8156847633 & 1.1508336974 & -5.7236611264 \\
\hline $\mathrm{C} 21$ & 0.5051420941 & 1.1826291198 & -5.4547168605 \\
\hline 022 & 2.9247619008 & $-\odot .0356087466$ & 2.5138489757 \\
\hline C23 & 2.7609907536 & -1.0926882742 & 0.4604569300 \\
\hline C24 & 2.5680627305 & -1.1702426314 & -1.0258106661 \\
\hline C25 & 1.7713280938 & -2.0945292963 & -1.6406934476 \\
\hline C26 & 2.4839321391 & -0.7776352378 & -4.9400230816 \\
\hline H27 & 0.0482693587 & 0.1609411972 & 3.2357953475 \\
\hline H28 & -1.7735602099 & -1.5061599299 & 3.4511362631 \\
\hline H29 & -2.6878342199 & -2.6159658420 & 1.4144337250 \\
\hline H3० & -1.8099891544 & -2.0135796178 & -0.8189548737 \\
\hline H31 & 1.2994344073 & 1.4913699628 & $-\odot .0023774682$ \\
\hline H32 & 1.2653312154 & 1.7836851991 & 1.7318649668 \\
\hline H33 & 2.9687290812 & $\odot .7853520715$ & 3.0235519480 \\
\hline H34 & 3.6496349365 & 1.5274762222 & -0.4021219436 \\
\hline H35 & 3.7372323408 & 2.1814785234 & 1.2226827385 \\
\hline H36 & 3.7635124570 & -1.4954603825 & $\odot .6597340693$ \\
\hline H37 & 2.0491066518 & -1.7452881674 & $\odot .9697819034$ \\
\hline H38 & 5.4698625507 & 0.3093176223 & 1.7836827665 \\
\hline H39 & 7.1282529308 & $\odot .2542822832$ & -0.0131252997 \\
\hline $\mathrm{H} 40$ & 5.9239005195 & 1.0404116947 & -1.1745195857 \\
\hline H41 & 3.2593889966 & -0.5893433533 & -1.6358288505 \\
\hline $\mathrm{H} 42$ & 1.8704282009 & -2.3186444786 & -2.6971349662 \\
\hline H43 & 1.1656543499 & -2.7750460358 & -1.0502262952 \\
\hline H44 & 1.1401151495 & 1.6258041553 & -6.2241468542 \\
\hline H45 & -1.1015389526 & 1.5517362657 & -6.6976847906 \\
\hline H46 & -3.2089056863 & 0.1155453472 & -3.3940128836 \\
\hline H47 & -2.7054314948 & 0.1526293698 & -5.6537888645 \\
\hline H48 & -3.4503080865 & 2.1420057925 & -1.3467885295 \\
\hline H49 & -3.0863990096 & 0.5022281489 & -0.7242518575 \\
\hline H50 & -2.2135010161 & 1.8912216567 & -0.0797263187 \\
\hline H51 & -1.8121479136 & 3.5963458641 & -2.8735947411 \\
\hline H52 & -0.4077681502 & 3.4510735385 & -1.7851761615 \\
\hline H53 & -0.2426332742 & 3.0679109126 & -3.5152339695 \\
\hline H54 & 2.9540448999 & $-\odot .3571032555$ & -5.8339414337 \\
\hline H55 & 3.2697445097 & -1.1497590483 & -4.2759802251 \\
\hline H56 & 1.8466705027 & -1.6147397595 & -5.2363027693 \\
\hline H57 & 3.1773498585 & 2.1462863878 & -4.7522356104 \\
\hline H58 & 2.2355924468 & 2.7235402402 & -3.3556795058 \\
\hline H59 & 3.4951233982 & 1.5011390667 & -3.1264733393 \\
\hline
\end{tabular}


Energy components, in hartrees:

(A) Nuclear repulsion.......... 3169.66130367412

(E) Total one-electron terms ..... -8717.75487683106

(I) Total two-electron terms.... 3805.39854827205

(J) Coulomb............. 4004.79720241299

(K) Exchange+Correlation....... -199.39865414094

(L) Electronic energy........... $-4912.35632855901 \quad(E+I)$

(N) Total energy............. -1742.69502488489 (A+L)

SCFE: SCF energy: DFT $\quad-1742.69502488489$ hartrees

HOMO energy: $-\odot .32408$

LUMO energy: $-\odot .18512$

Thermodynamic properties calculated assuming an ideal gas

In the table below, the units for temperature

are kelvins, the units for $\mathrm{U}, \mathrm{H}$, and $\mathrm{G}$ are

$\mathrm{kcal} / \mathrm{mol}$ and the units for $\mathrm{Cv}$ and $\mathrm{S}$ are $\mathrm{cal} /(\mathrm{mol} \mathrm{K})$

The zero point energy (ZPE): $312.119 \mathrm{kcal} / \mathrm{mol}$

is not included in $\mathrm{U}, \mathrm{H}$, or $\mathrm{G}$ in the table below

$\mathrm{T}=298.15 \mathrm{~K}$

\begin{tabular}{rrrrrr} 
& \multicolumn{1}{c}{ U } & \multicolumn{1}{c}{ CV } & \multicolumn{1}{c}{$\mathrm{S}$} & \multicolumn{1}{c}{$\mathrm{H}$} & \multicolumn{1}{c}{$\mathrm{G}$} \\
trans. & 0.889 & 2.981 & 44.401 & 1.481 & -11.757 \\
rot. & 0.889 & 2.981 & 35.779 & 0.889 & -9.779 \\
vib. & 17.838 & 113.334 & 114.635 & 17.838 & -16.340 \\
elec. & 0.000 & 0.000 & 0.000 & 0.000 & 0.000 \\
total & 19.616 & 119.296 & 194.814 & 20.208 & -37.876
\end{tabular}

Total internal energy, Utot (SCFE + ZPE + U): $\quad-1742.166371$ hartrees

Total enthalpy, Htot (Utot + pV): -1742.165427 hartrees

Total Gibbs free energy, Gtot (Htot - $T^{\star} S$ ): $\quad-1742.257990$ hartrees

\section{Single point calculation at the B3LYP/LACV3P**++//B3LYP/LACVP** level}

Energy components, in hartrees:

(A) Nuclear repulsion.......... 3169.66130367412

(E) Total one-electron terms..... -8717.55091920965

(I) Total two-electron terms.... 3804.91188871680

(J) Coulomb.............. 4004.36453264760

(K) Exchange+Correlation....... -199.45264393080

(L) Electronic energy............

(N) Total energy............ $-1742.97772681873 \quad(\mathrm{~A}+\mathrm{L})$

SCFE: SCF energy: DFT $\quad-1742.97772681873$ hartrees

HOMO energy: -0.33336

LUMO energy: $-\odot .19511$

B3LYP/LACV3P**++//B3LYP/LACVP** Total Gibbs free energy, Gtot (Htot - T*S): -1742.540692 hartrees 


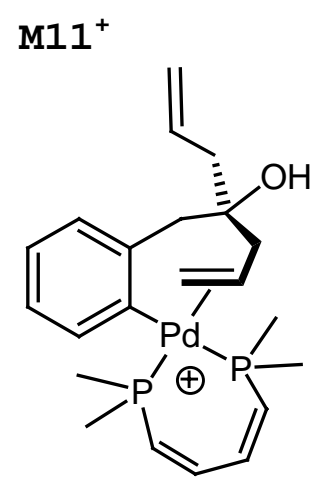

NIMAG $\odot$

\begin{tabular}{|c|c|c|c|}
\hline C1 & -1.1991212368 & ๑. 3929887122 & -2.2298357628 \\
\hline C2 & -1.2763192287 & 0.1330587171 & $-\odot .8590408178$ \\
\hline C3 & -0.1409403636 & $-\odot .3088232915$ & -0.1603288227 \\
\hline $\mathrm{C} 4$ & 1.0502005209 & -0.5308196320 & -0.8685551987 \\
\hline C5 & 1.1214663298 & -0.2994444394 & -2.2454342089 \\
\hline C6 & $-\odot .0 \odot \odot 2768909$ & 0.1759498579 & -2.9259804634 \\
\hline $\mathrm{Pd} 7$ & -2.9258608941 & ๑. 4352941135 & ๑. 3549706307 \\
\hline P8 & -3.7760701023 & -1.5324966751 & -0.6512999085 \\
\hline C9 & -4.5188980554 & -1.0339522671 & -2.2689644889 \\
\hline C10 & -0.2134971596 & -0.4761473636 & 1.3421939936 \\
\hline C11 & -0.1396424554 & ๑. 8436648717 & 2. 2003702819 \\
\hline C12 & -0.4582320825 & 2.1446580080 & 1.4132959488 \\
\hline C13 & -1.9190029971 & 2.4238597547 & 1.1505279952 \\
\hline C14 & -2.4777375548 & 2.7209892604 & -0.0614511852 \\
\hline C15 & 1.2754859373 & ๑. 9371923137 & 2.8245631742 \\
\hline C16 & 1.4742482892 & 2.0725322489 & 3. 7901773399 \\
\hline C17 & 2.5033593359 & 2.9200968011 & 3.7512545983 \\
\hline P18 & -4.8533110052 & ๑. 5540984020 & 1.8691237330 \\
\hline C19 & -6.5199204628 & $\odot .6787483186$ & 1. 0814499646 \\
\hline C20 & -4.8935817206 & 1.8801975323 & 3.1480507010 \\
\hline C21 & -4.8858962072 & $-\odot .9445972026$ & 2.9058949774 \\
\hline $\mathrm{C} 22$ & -5.1827440737 & -2.2104942144 & 2.5595102612 \\
\hline $\mathrm{C} 23$ & -5.5976221261 & -2.7674352728 & 1. 2669793412 \\
\hline C24 & -5.1478688278 & -2.5415198690 & ๑. 0189791197 \\
\hline C25 & -2.5895149091 & -2.8566957892 & -1.1457499882 \\
\hline H26 & 1.9277546134 & $-\odot .8883130491$ & -0.3343034584 \\
\hline $\mathrm{H} 27$ & 2.0496174078 & -0.4794328378 & -2.7795381441 \\
\hline H28 & ๑. . 0485091867 & ๑. 3791241942 & -3.9923135706 \\
\hline H29 & -2.0583111778 & $\odot .7787678456$ & -2.7728959944 \\
\hline H30 & -1.1639167237 & $-\odot .9617201938$ & 1.5998209511 \\
\hline H31 & $\odot .5813434915$ & -1.1534400157 & 1.6757093689 \\
\hline H32 & -3.1181101390 & -3.6455215960 & -1.6892099548 \\
\hline H33 & -1.8156743392 & -2.4248186628 & -1.7824533593 \\
\hline H34 & -2.1206651086 & -3.2860622865 & $-\odot .2577267554$ \\
\hline H35 & -3.7512330383 & -0.5753696940 & -2.8935886138 \\
\hline H36 & -5.3205210769 & $-\odot .3095398781$ & -2.1081021509 \\
\hline H37 & -4.9227792065 & -1.9077385587 & -2.7894327088 \\
\hline H38 & -5.7591244412 & 1.7627089804 & 3.8063058410 \\
\hline H39 & -3.9766462686 & 1.8341564508 & 3.7417660074 \\
\hline $\mathrm{H} 40$ & -4.9498206093 & 2.8593751947 & 2.6645569987 \\
\hline H41 & -7.3115194279 & ๑. 6360999881 & 1.8340568966 \\
\hline $\mathrm{H} 42$ & -6.5826888154 & 1.6296361659 & 0.5445769415 \\
\hline H43 & -6.6656403001 & -0.1344831171 & ๑. 3686970517 \\
\hline H44 & -4.6032814120 & -0.7663849411 & 3.9424888347 \\
\hline H45 & -5.1796589477 & -2.9490508172 & 3.3618716497 \\
\hline H46 & -5.6071960878 & -3.1359572138 & -0.7733415537 \\
\hline $\mathrm{H} 47$ & -6.3637101017 & -3.5397750559 & 1.3561565724 \\
\hline H48 & $-\odot .0917974227$ & 2.9784395582 & 2.0266376774 \\
\hline H49 & ๑.1141167704 & 2.1529678214 & ๑. 4832710828 \\
\hline $\mathrm{H} 50$ & 1.4526061363 & -0.0169513496 & 3. 3472495912 \\
\hline H51 & 2.0184397770 & ๑. 9811111405 & 2.0184753042 \\
\hline H52 & ๑. 7260707666 & 2.1636673659 & 4.5770939364 \\
\hline H53 & 2.6254054805 & 3.7032442285 & 4.4926920118 \\
\hline H54 & 3.2705129056 & 2.8498122570 & 2.9824609021 \\
\hline H55 & -2.5147132598 & 2.5784120268 & 2.0442070830 \\
\hline H56 & -3.4845931344 & 3.1238743785 & -0.1257238405 \\
\hline H57 & -1.8862713874 & 2.7340380198 & -0.9699919717 \\
\hline 058 & -1.1278752117 & $\odot .7826543430$ & 3.2469935800 \\
\hline H59 & -0.8882360279 & ๑. . 0562589299 & 3.8389481235 \\
\hline
\end{tabular}


Energy components, in hartrees:

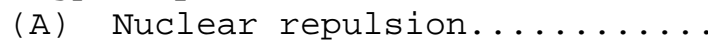
3191.92373511352
(E) Total one-electron terms.....
$-8762.68104865129$
(I) Total two-electron terms.....
3828.06526956603
(J) Coulomb............... 4. 4027.46769781930
(K) Exchange+Correlation . . . . . $\quad-199.40242825327$
(L) Electronic energy........... -4934.61577908526

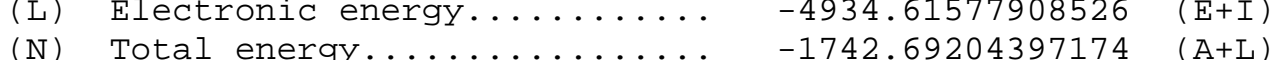

SCFE: SCF energy: DFT $\quad-1742.69204397174$ hartrees

HOMO energy: $\quad-0.32717$

LUMO energy: -0.18257

Thermodynamic properties calculated assuming an ideal gas

In the table below, the units for temperature

are kelvins, the units for $U, H$, and $G$ are

$\mathrm{kcal} / \mathrm{mol}$ and the units for $\mathrm{Cv}$ and $\mathrm{S}$ are $\mathrm{cal} /(\mathrm{mol} \mathrm{K})$

The zero point energy (ZPE): $312.417 \mathrm{kcal} / \mathrm{mol}$

is not included in $U, H$, or $G$ in the table below

$\mathrm{T}=298.15 \mathrm{~K}$

\begin{tabular}{|c|c|c|c|c|c|}
\hline & $U$ & CV & $\mathrm{S}$ & $\mathrm{H}$ & G \\
\hline & -------- & $-----\cdot--$ & ------- & 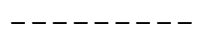 & ------ \\
\hline trans. & 0.889 & 2.981 & 44.401 & 1.481 & -11.757 \\
\hline rot. & 0.889 & 2.981 & 35.738 & 0.889 & -9.767 \\
\hline vib. & 17.817 & 113.186 & 116.523 & 17.817 & -16.924 \\
\hline elec. & 0.000 & 0.000 & 0.000 & 0.000 & $0.00 \odot$ \\
\hline total & 19.595 & 119.147 & 196.662 & 20.187 & -38.447 \\
\hline
\end{tabular}

Total internal energy, Utot (SCFE + ZPE + U): -1742.162949 hartrees Total enthalpy, Htot (Utot + pV): -1742.162005 hartrees Total Gibbs free energy, Gtot (Htot - $\mathrm{T}^{\star} \mathrm{S}$ ): $\quad-1742.255445$ hartrees

\section{Single point calculation at the B3LYP/LACV3P**++//B3LYP/LACVP** level}

Energy components, in hartrees:
(A) Nuclear repulsion.......... 3191.92373511352
(E) Total one-electron terms.... -8762.55775482067
(I) Total two-electron terms.... 3827.66007012161
(J) Coulomb.............. 4027.11949314817
(K) Exchange+Correlation...... $\quad-199.45942302656$
(L) Electronic energy.......... -4934.89768469906 (E+I)
(N) Total energy............. 1742.97394958554 (A+L)

SCFE: SCF energy: DFT $\quad-1742.97394958554$ hartrees

HOMO energy: $\quad-0.33649$

LUMO energy: $\quad-0.19163$

B3LYP/LACV3P**++//B3LYP/LACVP** Total Gibbs free energy, Gtot (Htot - $\mathrm{T}^{*} \mathrm{~S}$ ): -1742.537351 hartrees 


\section{$\mathrm{M}^{+} 2^{+}$}

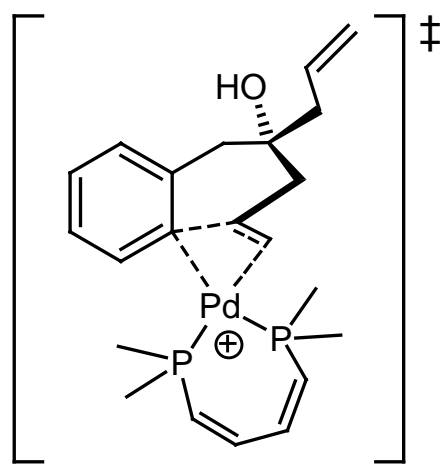

NIMAG 1

\begin{tabular}{|c|c|c|c|}
\hline C1 & $\odot .6007490000$ & -1.1434420000 & -1.6830410000 \\
\hline $\mathrm{C} 2$ & $\odot .3265770000$ & $-\odot .9203100000$ & -3.0341950000 \\
\hline C3 & -0.7426470000 & $-\odot .0945430000$ & $-3.400259000 \odot$ \\
\hline $\mathrm{C} 4$ & -1.5287280000 & ๑. 4984370000 & -2.4088940000 \\
\hline C5 & -1.2803170000 & $\odot .239689000 \odot$ & -1.0505940000 \\
\hline C6 & $-\odot .1941600000$ & $-\odot .571632000 \odot$ & $-\odot .677114000 \odot$ \\
\hline $\mathrm{Pd} 7$ & -2.9776090000 & $\odot .415370000 \odot$ & ๑. 2852280000 \\
\hline P8 & -3.8149760000 & -1.6768510000 & $-0.581997000 \odot$ \\
\hline C9 & -2.6071400000 & -2.9464840000 & -1.1654850000 \\
\hline C10 & $\odot .1216180000$ & $-\odot .814842000 \odot$ & $\odot .7831670000$ \\
\hline C11 & ๑ . $2919030 \odot \odot \odot$ & $\odot .4768660000$ & 1.6581140000 \\
\hline 012 & $-\odot .7777510000$ & ๑. 5885310000 & 2.6166660000 \\
\hline C13 & -2.2717130000 & 2.3805960000 & $\odot .814232000 \odot$ \\
\hline C14 & -1.0944400000 & 2.0509390000 & 0.1006440000 \\
\hline C15 & $\odot .2187720000$ & 1. 7530260000 & ๑. 8003310000 \\
\hline P16 & -4.7872250000 & $\odot .575784000 \odot$ & $1.814051000 \odot$ \\
\hline C17 & -6.4980970000 & 0.5030850000 & 1.1271850000 \\
\hline C18 & $1.645883000 \odot$ & $\odot .4109820000$ & 2.4106860000 \\
\hline C19 & $1.893323000 \odot$ & 1.5548580000 & 3.3568220000 \\
\hline $\mathrm{C} 20$ & 2.9745940000 & 2.3356700000 & 3.3312280000 \\
\hline $\mathrm{C} 21$ & -4.8653000000 & 2.0283640000 & $2.943971000 \odot$ \\
\hline $\mathrm{C} 22$ & -4.6381300000 & $-\odot .8021670000$ & 2.9947520000 \\
\hline $\mathrm{C} 23$ & $-4.852688000 \odot$ & $-2.11741100 \odot \odot$ & 2.8029450000 \\
\hline C24 & -5.3112990000 & -2.8563000000 & 1.6199690000 \\
\hline $\mathrm{C} 25$ & -5.0029950000 & -2.7453930000 & ๑. 3130020000 \\
\hline C26 & -4.7695550000 & -1.3129190000 & -2.1226130000 \\
\hline $\mathrm{H} 27$ & $-\odot .9862760000$ & 2.5240920000 & $-\odot .868762000 \odot$ \\
\hline H28 & $1.44573400 \odot \odot$ & $-1.7673800 \odot \odot \odot$ & $-1.40017100 \odot \odot$ \\
\hline $\mathrm{H} 29$ & $\odot .956184000 \odot$ & $-1.369602000 \odot$ & -3.7965080000 \\
\hline H30 & $-\odot .9515420000$ & $\odot .1019930000$ & -4.4481000000 \\
\hline H31 & $-2.335215000 \odot$ & $1.170 \odot \odot 20 \odot \odot \odot$ & $-2.695600000 \odot$ \\
\hline H32 & -0.6884140000 & -1.4029350000 & 1. 2324710000 \\
\hline H33 & 1.0275300000 & -1.4253200000 & $\odot .8522770000$ \\
\hline H34 & -3.1363350000 & -3.7957730000 & $-1.608260000 \odot$ \\
\hline H35 & -1.9436560000 & -2.5040010000 & -1.9108410000 \\
\hline H36 & $-2 . \odot \odot 72080 \odot \odot \odot$ & $-3.30056200 \odot \odot$ & -0.3243720000 \\
\hline H37 & -4.1017890000 & -0.8294220000 & -2.8393600000 \\
\hline H38 & -5.5962090000 & $-\odot .632882000 \odot$ & -1.9036040000 \\
\hline H39 & -5.1663550000 & -2.2308840000 & -2.5672010000 \\
\hline $\mathrm{H} 40$ & -5.7117260000 & 1.9367660000 & 3.6309480000 \\
\hline H41 & -3.9406680000 & 2. $.99869000 \odot$ & $3.522025000 \odot$ \\
\hline $\mathrm{H} 42$ & -4.9791820000 & $2.945219000 \odot$ & $2.359999000 \odot$ \\
\hline H43 & -7.2347170000 & $\odot .5160800000$ & 1.9354100000 \\
\hline H44 & $-6.654654000 \odot$ & $1.37173100 \odot \odot$ & $\odot .4813080000$ \\
\hline H45 & -6.6352310000 & -0.4044190000 & 0.5376790000 \\
\hline H46 & -4.3063010000 & $-\odot .4928360000$ & 3.9854410000 \\
\hline $\mathrm{H} 47$ & $-4.7347950 \odot \odot \odot$ & $-2.7546770 \odot \odot \odot$ & 3.6804860000 \\
\hline H48 & -5.4730410000 & -3.4734140000 & -0.3510310000 \\
\hline H49 & $-5.979672000 \odot$ & $-3.680874000 \odot$ & 1.8745200000 \\
\hline H50 & 0.4378050000 & 2.6076540000 & 1.4516080000 \\
\hline H51 & 1.0146310000 & 1.7012260000 & $\odot .0520250000$ \\
\hline H52 & 1.6532160000 & $-\odot .5391950 \odot \odot \odot$ & $2.969849000 \odot$ \\
\hline H53 & 2.4597220000 & $\odot .3393300000$ & 1.6788480000 \\
\hline H54 & 1.1220370000 & 1.7277410000 & 4.1056530000 \\
\hline H55 & 3.1136260000 & 3.1388370000 & 4.0483330000 \\
\hline H56 & 3.7665620000 & 2.1928200000 & 2.5991050000 \\
\hline H57 & -0.7170630000 & -0.1678800000 & 3. 2163150000 \\
\hline
\end{tabular}




$\begin{array}{llll}\text { H58 } & -2.9700130000 & 3.0737430000 & 0.3497410000 \\ \text { H59 } & -2.225011000 \odot & 2.38543000 \odot \odot & 1.896259000 \odot\end{array}$

Energy components, in hartrees:
(A) Nuclear repulsion............
3196.68326606573
(E) Total one-electron terms.....
$-8772.49970075533$
(I) Total two-electron terms.....
3833.13445820554
(J) Coulomb............. 4032.53969691400
(K) Exchange+Correlation....... $\quad-199.40523870846$
(L) Electronic energy........... -4939.36524254979
(N) Total energy............ -1742.68197648406

SCFE: SCF energy: DFT $\quad-1742.68197648406$ hartrees

HOMO energy: $\quad-\odot .32456$

LUMO energy: -0.17311

Thermodynamic properties calculated assuming an ideal gas

In the table below, the units for temperature

are kelvins, the units for $\mathrm{U}, \mathrm{H}$, and $\mathrm{G}$ are

$\mathrm{kcal} / \mathrm{mol}$ and the units for $\mathrm{Cv}$ and $\mathrm{S}$ are $\mathrm{cal} /(\mathrm{mol} \mathrm{K})$

The zero point energy (ZPE): $\quad 312.175 \mathrm{kcal} / \mathrm{mol}$ is not included in $U, H$, or $G$ in the table below

$\mathrm{T}=298.15 \mathrm{~K}$

\begin{tabular}{rrrrrr} 
& \multicolumn{1}{c}{ U } & \multicolumn{1}{c}{ CV } & \multicolumn{1}{c}{ S } & \multicolumn{1}{c}{ H } & \multicolumn{1}{c}{ G } \\
trans. & 0.889 & 2.981 & 44.401 & 1.481 & -11.757 \\
rot. & 0.889 & 2.981 & 35.750 & 0.889 & -9.770 \\
vib. & 16.724 & 108.947 & 104.734 & 16.724 & -14.503 \\
elec. & 0.000 & 0.000 & 0.000 & 0.000 & 0.000 \\
total & 18.501 & 114.909 & 184.885 & 19.094 & -36.030
\end{tabular}

Total internal energy, Utot (SCFE + ZPE + U): $\quad-1742.155010$ hartrees Total enthalpy, Htot (Utot + pV): -1742.154066 hartrees Total Gibbs free energy, Gtot (Htot - $T^{\star} S$ ): -1742.241911 hartrees

\section{Single point calculation at the B3LYP/LACV3P**++//B3LYP/LACVP** level}

Energy components, in hartrees:
(A) Nuclear repulsion.......... 3196.68326606573
(E) Total one-electron terms..... -8772.40506380738
(I) Total two-electron terms .... 3832.75884338640
(J) Coulomb............ 4032.22292515788
(K) Exchange+Correlation...... $\quad-199.46408177148$
(L) Electronic energy........... $-4939.64622042098 \quad(\mathrm{E}+\mathrm{I})$
(N) Total energy............. $-1742.96295435526 \quad(\mathrm{~A}+\mathrm{L})$

SCFE: SCF energy: DFT $\quad-1742.96295435526$ hartrees

HOMO energy: $\quad-0.33297$

LUMO energy: $-\odot .18315$

B3LYP/LACV3P**++//B3LYP/LACVP** Total Gibbs free energy, Gtot (Htot - T*S): -1742.522889 hartrees 


\section{$\mathrm{M}^{+} 3^{+}$}<smiles>C=CC[C@]1(O)Cc2ccccc2[C@@]2(I)C[C@@](I)(P3(C)(C)C=CC=CP3(C)(C)C)[P+]2(C)C1</smiles>

\section{NIMAG $\odot$}

\begin{tabular}{|c|c|c|c|}
\hline $\mathrm{C} 1$ & ๑. 3272897059 & -1.3175548235 & $-\odot .9913858114$ \\
\hline $\mathrm{C} 2$ & -0.0592673302 & -1.2283859154 & -2.3324422163 \\
\hline C3 & $-\odot .8657121226$ & $-\odot .1754975925$ & -2.7631653488 \\
\hline $\mathrm{C} 4$ & -1.2649714298 & $\odot .8051541984$ & -1.8510115232 \\
\hline C5 & $-\odot .8592384273$ & $\odot .7425583661$ & -0.5030284658 \\
\hline C6 & $-\odot .0681041743$ & $-\odot .3511511074$ & $-\odot .0641020035$ \\
\hline $\mathrm{Pd} 7$ & -3.1480231532 & ๑.1328834775 & ๑. 3211328363 \\
\hline P8 & -4.0930586751 & -1.7913770360 & -0.9911296535 \\
\hline C9 & -3.0180002992 & -3.2969038043 & -1.0908977521 \\
\hline C10 & ๑. 3582292298 & -0.4037722836 & 1.3838253432 \\
\hline C11 & $\odot .8285365270$ & $\odot .9640264247$ & 1.9867465275 \\
\hline 012 & ๑. 2711181518 & 1.1125651766 & 3.3042781276 \\
\hline C13 & -2.2390106302 & 1.6886563004 & 1.3596207990 \\
\hline C14 & -1.0374848156 & 1.9363024092 & $\odot .4516375308$ \\
\hline C15 & ๑. 3051599476 & 2.1680124063 & 1.1763951926 \\
\hline P16 & -4.9701662504 & ๑. 2571978011 & 1.7114910558 \\
\hline $\mathrm{C} 17$ & -6.5335698658 & 0.7253074590 & $\odot .8545613934$ \\
\hline C18 & 2.3769237582 & $\odot .9822201970$ & 2.0663306770 \\
\hline C19 & 2.9619365296 & 2.2688311163 & 2.5788006890 \\
\hline $\mathrm{C} 2 \mathrm{O}$ & 3.8801354843 & 2.9915739803 & 1.9349722526 \\
\hline $\mathrm{C} 21$ & -4.9250546934 & 1.4004219950 & 3.1503926252 \\
\hline $\mathrm{C} 22$ & -5.2471112881 & -1.3441846836 & 2.5223119003 \\
\hline $\mathrm{C} 23$ & -5.7518938773 & -2.4827980141 & 2.0145027631 \\
\hline C24 & -6.2424998791 & -2.7950354726 & ๑.6665183803 \\
\hline $\mathrm{C} 25$ & -5.7029700716 & -2.5515065061 & $-\odot .5417063721$ \\
\hline $\mathrm{C} 26$ & -4.4227774973 & -1.4060429363 & -2.7686626403 \\
\hline $\mathrm{H} 27$ & -1.2435095307 & 2.8145794861 & -0.1688354710 \\
\hline $\mathrm{H} 28$ & $\odot .9663855335$ & -2.1353404453 & $-\odot .6686227382$ \\
\hline $\mathrm{H} 29$ & $\odot .2861278676$ & -1.9738255406 & -3.0432535952 \\
\hline H3O & -1.1489048809 & $-\odot .0930756647$ & -3.8084726550 \\
\hline H31 & -1.8330617338 & 1.6672478785 & -2.1925688489 \\
\hline H32 & $-\odot .4989879525$ & $-\odot .7242943407$ & 1.9842328280 \\
\hline H33 & 1.1311393776 & -1.1662899998 & 1.5169090009 \\
\hline H34 & -3.4743604623 & -4.0654756373 & -1.7211906073 \\
\hline H35 & -2.0468549139 & -3.0156102178 & -1.5041872188 \\
\hline H36 & -2.8681022004 & -3.7009656427 & $-\odot .0868770385$ \\
\hline H37 & -3.4956049753 & -1.0783956430 & -3.2421050282 \\
\hline H38 & -5.1543175650 & $-\odot .5967476476$ & -2.8431616672 \\
\hline H39 & -4.8051939758 & -2.2830178631 & -3.2998580330 \\
\hline $\mathrm{H} 40$ & -5.8335472930 & 1.2781425853 & 3.7471305778 \\
\hline H41 & -4.0526623476 & 1.1930662707 & 3.7742452651 \\
\hline $\mathrm{H} 42$ & -4.8653388572 & 2.4347259163 & 2.8054204009 \\
\hline $\mathrm{H} 43$ & -7.3665135954 & 0.7076081965 & 1.5636565081 \\
\hline $\mathrm{H} 44$ & -6.4237691942 & 1.7345396930 & $\odot .4491253022$ \\
\hline H45 & -6.7457773434 & ๑. . 0355690399 & ๑. . 0372129518 \\
\hline H46 & -4.9488226164 & $-1.3510 \odot 44365$ & 3.5698438379 \\
\hline $\mathrm{H} 47$ & -5.8831473238 & -3.3048380885 & 2.7199791704 \\
\hline H48 & -6.2534250451 & -2.9333747858 & -1.4025562895 \\
\hline H49 & -7.1610767250 & -3.3847428888 & $\odot .6726207640$ \\
\hline H50 & ๑.2337924863 & 3.0227435439 & 1.8548953575 \\
\hline H51 & 1.0455222985 & 2.4307559957 & 0.4119983103 \\
\hline H52 & 2.6769624947 & 0.1493335573 & 2.7238734107 \\
\hline H53 & 2.7861184537 & ๑. 7513193879 & 1.0750648017 \\
\hline H54 & 2.5902094862 & 2.6061357808 & 3.5453941469 \\
\hline H55 & 4.2785487281 & 3.9107093462 & 2.3539186829 \\
\hline H56 & 4.2779499213 & 2.6870795555 & ๑. 9688281866 \\
\hline H57 & 0.6635671584 & 0.4373843679 & 3.8765870318 \\
\hline H58 & -2.8978704268 & 2.5519880543 & 1.4467877956 \\
\hline
\end{tabular}


$\begin{array}{llll}\text { H59 } & -1.9715065666 & 1.3034702112 & 2.3443872831\end{array}$

Energy components, in hartrees:
(A) Nuclear repulsion...........
3119.61526029298
(E) Total one-electron terms....
$-8618.54545744907$
(I) Total two-electron terms..... 3756.21063124693
(J) Coulomb................ 3955.67889765900
(K) Exchange+Correlation...... $\quad-199.46826641207$
(L) Electronic energy. . . . . . . . . . -4862.33482620215
(N) Total energy............... -1742.71956590917
$(E+I)$
$(A+L)$

SCFE: SCF energy: DFT

-1742.71956590917 hartrees

$\begin{array}{ll}\text { HOMO energy: } & -0.32562 \\ \text { LUMO energy: } & -0.18650\end{array}$

Thermodynamic properties calculated assuming an ideal gas

In the table below, the units for temperature are kelvins, the units for $U, H$, and $G$ are

$\mathrm{kcal} / \mathrm{mol}$ and the units for $\mathrm{Cv}$ and $\mathrm{S}$ are cal/(mol K)

The zero point energy (ZPE): $313.480 \mathrm{kcal} / \mathrm{mol}$

is not included in $U, H$, or $G$ in the table below

$\mathrm{T}=298.15 \mathrm{~K}$

\begin{tabular}{rrrrrr} 
& \multicolumn{1}{c}{ U } & \multicolumn{1}{c}{ CV } & \multicolumn{1}{c}{ S } & \multicolumn{1}{c}{ H } & \multicolumn{1}{c}{ G } \\
trans. & 0.889 & 2.981 & 44.401 & 1.481 & -11.757 \\
rot. & 0.889 & 2.981 & 35.955 & 0.889 & -9.831 \\
vib. & 17.546 & 111.216 & 116.331 & 17.546 & -17.138 \\
elec. & 0.000 & 0.000 & 0.000 & 0.000 & 0.000 \\
total & 19.324 & 117.178 & 196.687 & 19.916 & -38.726
\end{tabular}

Total internal energy, Utot (SCFE + ZPE + U): -1742.189209 hartrees Total enthalpy, Htot (Utot + pV): -1742.188265 hartrees Total Gibbs free energy, Gtot (Htot - $\mathrm{T}^{\star} \mathrm{S}$ ): -1742.281718 hartrees

\section{Single point calculation at the B3LYP/LACV3P**++//B3LYP/LACVP** level}

Energy components, in hartrees:

(A) Nuclear repulsion.......... 3119.61526029298

(E) Total one-electron terms.... -8618.43708273615

(I) Total two-electron terms..... 3755.82315533868

(J) Coulomb.............. 3955.34457476432

(K) Exchange+Correlation...... $\quad-199.52141942564$

(L) Electronic energy.......... -4862.61392739747 (E+I)

(N) Total energy............. $-1742.99866710450 \quad(\mathrm{~A}+\mathrm{L})$

SCFE: SCF energy: DFT $\quad-1742.99866710450$ hartrees

HOMO energy: $\quad-0.33717$

LUMO energy: -0.19432

B3LYP/LACV3P**++//B3LYP/LACVP** Total Gibbs free energy, Gtot (Htot - $T^{*} S$ ): -1742.560819 hartrees 


\section{M14}

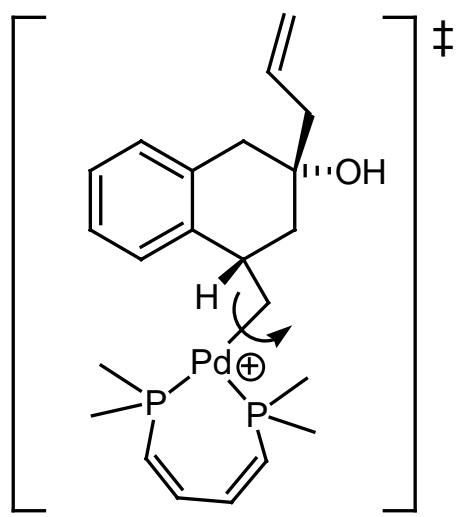

NIMAG 1

\begin{tabular}{|c|c|c|c|}
\hline Pd1 & -3.1172760000 & $\odot .1695480000$ & ๑. 2709390000 \\
\hline P2 & -4.0627040000 & -1.8277780000 & $-0.916217000 \odot$ \\
\hline C3 & -2.9601640000 & -3.3110080000 & -1.0351760000 \\
\hline C4 & -4.4802780000 & -1.4836530000 & -2.6836680000 \\
\hline C5 & -5.6306560000 & -2.6083140000 & -0.3625920000 \\
\hline C6 & $-6.107074000 \odot$ & $-2.815857000 \odot$ & ๑.87991600०๑ \\
\hline $\mathrm{H} 7$ & -6.7070020000 & -0.0120650000 & $\odot .1423490000$ \\
\hline $\mathrm{C} 8$ & -5.5591110000 & -2.4368600000 & 2.1903700000 \\
\hline $\mathrm{C9}$ & -5.0685110000 & -1.2619280000 & 2.6275760000 \\
\hline P10 & -4.8829290000 & 0.3130610000 & $1.740358000 \odot$ \\
\hline C11 & -6.4886110000 & $\odot .7085680000$ & $\odot .9306110000$ \\
\hline C12 & -4.7943440000 & 1.5253530000 & 3.1190230000 \\
\hline C13 & -2.2360900000 & 1.8188870000 & 1.1697990000 \\
\hline C14 & -1.0852900000 & 1.7497740000 & $\odot .16844400 \odot \odot$ \\
\hline C15 & $-\odot .75263400 \odot \odot$ & 3. $.834500 \odot \odot \odot$ & -0.5294930000 \\
\hline C16 & $-\odot .220567000 \odot$ & 4.1933380000 & ๑. 3959070000 \\
\hline 017 & -1.3821480000 & 4.7967370000 & $\odot .997505000 \odot$ \\
\hline C18 & $\odot .5407940000$ & 5.2659280000 & -0.4235030000 \\
\hline C19 & $-\odot .268099000 \odot$ & 5.8818230000 & -1.5331370000 \\
\hline $\mathrm{C} 20$ & 0.1087200000 & 5.9191850000 & -2.8117420000 \\
\hline C21 & ๑.7144180000 & 3.6299170000 & 1.5216570000 \\
\hline $\mathrm{C} 22$ & 1.1102720000 & 2.1739890000 & 1.3943360000 \\
\hline C23 & 2.3866240000 & 1.7363970000 & 1.7663080000 \\
\hline C24 & 2.7531380000 & $\odot .391737000 \odot$ & 1.6470440000 \\
\hline $\mathrm{C} 25$ & 1.8345550000 & $-\odot .547688000 \odot$ & $1.171573000 \odot$ \\
\hline C26 & $\odot .5603190000$ & -0.1260130000 & 0.7826300000 \\
\hline $\mathrm{C} 27$ & $\odot .1898940000$ & 1.2334110000 & $\odot .863661000 \odot$ \\
\hline $\mathrm{H} 28$ & $-4.7283030 \odot \odot \odot$ & $2.539203000 \odot$ & $2.71827700 \odot \odot$ \\
\hline H29 & -5.6890240000 & 1.4456060000 & $3.743293000 \odot$ \\
\hline H30 & -3.9082370000 & 1. 3383940000 & 3.7297990000 \\
\hline H31 & -7.2967700000 & ๑. 6920040000 & 1.6675430000 \\
\hline H32 & -6.4178600000 & 1.7066490000 & 0.4903360000 \\
\hline H33 & -4.7156030000 & -1.2168960000 & 3.6572480000 \\
\hline H34 & -5.6250990000 & -3.2345950000 & 2.9320640000 \\
\hline H35 & $-7.008791000 \odot$ & -3.4266860000 & ๑. $953101000 \odot$ \\
\hline H36 & -6.2102100000 & -3.0381640000 & -1.1808590000 \\
\hline H37 & -2.7535200000 & -3.6863000000 & $-\odot .029740000 \odot$ \\
\hline H38 & -3.4271090000 & -4.1048260000 & $-1.625420000 \odot$ \\
\hline H39 & -2.0167470000 & -3.0150390000 & -1.4996880000 \\
\hline $\mathrm{H} 40$ & -5.2227660000 & $-\odot .6829030000$ & -2.7392850000 \\
\hline H41 & -3.5784010000 & -1.1517010000 & -3.2022620000 \\
\hline $\mathrm{H} 42$ & $-4.8748850 \odot \odot \odot$ & $-2.37457600 \odot \odot$ & $-3.18220600 \odot \odot$ \\
\hline H43 & $-\odot .5089490000$ & 6.3845600000 & -3.5740670000 \\
\hline H44 & 1.0543410000 & 5.4945360000 & -3.1423120000 \\
\hline H45 & -1.2218670000 & 6.3169510000 & -1.2386090000 \\
\hline H46 & 0.8570700000 & 6.0502240000 & 0.2845680000 \\
\hline $\mathrm{H} 47$ & 1.4617780000 & 4.8250670000 & -0.8240730000 \\
\hline H48 & -1.0944240000 & 5.5891060000 & 1.4720760000 \\
\hline H49 & 1.6188800000 & 4. 2418040000 & 1.6094300000 \\
\hline H50 & $\odot .1658100000$ & 3.7525940000 & 2.4632970000 \\
\hline H51 & $\odot .0 \odot 7270000 \odot$ & $2.875587000 \odot$ & $-1.292443000 \odot$ \\
\hline H52 & $-1.6325910 \odot \odot \odot$ & 3. $47014300 \odot \odot$ & $-1.050551000 \odot$ \\
\hline H53 & -1.3363660000 & 1. 0170540000 & -0.6066760000 \\
\hline H54 & -2.6036400000 & 2.7361110000 & ๑. 7064090000 \\
\hline H55 & -2.4761760000 & 1.7813600000 & 2.2308370000 \\
\hline
\end{tabular}




$\begin{array}{rrrr}\text { H56 } & -0.136342000 \odot & -0.83494800 \odot \odot & 0.341461000 \odot \\ \text { H57 } & 2.119775000 \odot & -1.589766000 \odot & 1.0618360000 \\ \text { H58 } & 3.759964000 \odot & 0.0824690 \odot \odot \odot & 1.91308600 \odot \odot \\ \text { H59 } & 3.110839000 \odot & 2.460778000 \odot & 2.1307480000\end{array}$

Energy components, in hartrees:
(A) Nuclear repulsion...........
3009.92188606311
(E) Total one-electron terms.....
$-8399.79010680112$
(I) Total two-electron terms.....
3647.15603366214
(J) Coulomb............. 3846.61700424491
(K) Exchange+Correlation....... $\quad-199.46097058277$
(L) Electronic energy.......... -4752.63407313898
(N) Total energy............. -1742.71218707587

SCFE: SCF energy: DFT

-1742.71218707587 hartrees

HOMO energy: $-\odot .32493$

LUMO energy: -0.19471

Thermodynamic properties calculated assuming an ideal gas

In the table below, the units for temperature are kelvins, the units for $\mathrm{U}, \mathrm{H}$, and $\mathrm{G}$ are

$\mathrm{kcal} / \mathrm{mol}$ and the units for $\mathrm{CV}$ and $\mathrm{S}$ are $\mathrm{cal} /(\mathrm{mol} \mathrm{K})$

The zero point energy (ZPE): $312.405 \mathrm{kcal} / \mathrm{mol}$

is not included in $U, H$, or $G$ in the table below

$\mathrm{T}=298.15 \mathrm{~K}$

\begin{tabular}{rrrrrr} 
& \multicolumn{1}{c}{ U } & \multicolumn{1}{c}{ CV } & \multicolumn{1}{c}{ S } & \multicolumn{1}{c}{ H } & \multicolumn{1}{c}{ G } \\
trans. & 0.889 & 2.981 & 44.401 & 1.481 & -11.757 \\
rot. & 0.889 & 2.981 & 36.336 & 0.889 & -9.945 \\
vib. & 17.134 & 109.522 & 111.607 & 17.134 & -16.141 \\
elec. & 0.000 & 0.000 & 0.000 & 0.000 & 0.000 \\
total & 18.912 & 115.483 & 192.344 & 19.504 & -37.843
\end{tabular}

Total internal energy, Utot (SCFE + ZPE + U): $\quad-1742.184200$ hartrees Total enthalpy, Htot (Utot + pV): -1742.183256 hartrees Total Gibbs free energy, Gtot (Htot - $T^{\star} S$ ): $\quad-1742.274645$ hartrees

\section{Single point calculation at the B3LYP/LACV3P**++//B3LYP/LACVP** level}

Energy components, in hartrees:
(A) Nuclear repulsion.......... 3009.92188606311
(E) Total one-electron terms.... -8399.66036148607
(I) Total two-electron terms..... 3646.74509443736
(J) Coulomb............. 3846.25386951659
(K) Exchange+Correlation....... -199.50877507922
(L) Electronic energy.......... -4752.91526704871
(N) Total energy ............ $-1742.99338098560 \quad(\mathrm{~A}+\mathrm{L})$

SCFE: SCF energy: DFT $\quad-1742.99338098560$ hartrees

$\begin{array}{ll}\text { HOMO energy: } & -0.33515 \\ \text { LUMO energy: } & -0.20127\end{array}$

B3LYP/LACV3P**++//B3LYP/LACVP** Total Gibbs free energy, Gtot (Htot - T*S): -1742.555839 hartrees 


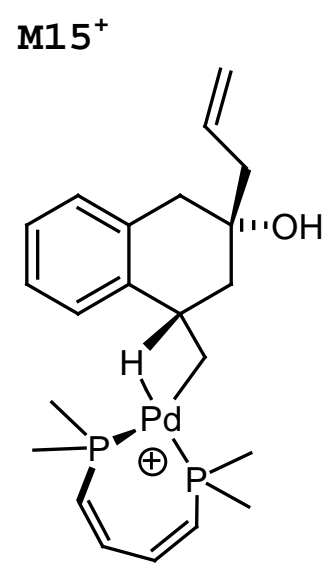

\section{NIMAG $\odot$}

\begin{tabular}{|c|c|c|c|}
\hline C1 & -1.8901549696 & 0.1952082879 & $-\odot .7545651871$ \\
\hline $\mathrm{C} 2$ & -1.4311850485 & -1.1303396816 & -1.3457069865 \\
\hline C3 & $-\odot .2219858236$ & -1.7083995835 & $-\odot .9256038609$ \\
\hline C4 & ๑. 7093915426 & -1.0164722013 & ๑. . 0522873116 \\
\hline C5 & ๑. . 0727094094 & ๑. 1325888295 & ๑. 8423105417 \\
\hline $\mathrm{C} 6$ & -0.7507260563 & 1.0161040384 & -0.1231599437 \\
\hline C7 & $\odot .1442855616$ & -2.9549997180 & -1.4555479829 \\
\hline C8 & $-\odot .6561760356$ & -3.6137876314 & -2.3860650633 \\
\hline C9 & -1.8534918328 & -3.0286273911 & -2.8089722766 \\
\hline C10 & -2.2301962643 & -1.7936880930 & -2.2866312412 \\
\hline 011 & -0.7879952309 & $-\odot .4842831854$ & 1.8029370949 \\
\hline C12 & 1.1755651714 & ๑. 9688114327 & 1.5485941457 \\
\hline C13 & ๑. 6507187939 & 1.9335567681 & 2.5810209198 \\
\hline C14 & ๑.6080296261 & 3. 2607156629 & 2.4379519392 \\
\hline C15 & -3.1395953309 & 0.1078003552 & ๑. 0625591555 \\
\hline Pd16 & -4.0324259164 & 1.0771281783 & -1.5545480349 \\
\hline P17 & -6.1906338872 & 1.1271021063 & $-\odot .7012005902$ \\
\hline C18 & -6.6155990211 & - ๑. 0913825299 & 0.6085464904 \\
\hline P19 & -4.5432936863 & 2.2065870882 & -3.6546500226 \\
\hline $\mathrm{C} 20$ & -3.2632973552 & 3. 3873931152 & -4.2658424614 \\
\hline C21 & -6.0465744767 & 3. 2543787018 & -3.6930212772 \\
\hline $\mathrm{C} 22$ & -7.3137297121 & 2.8960961066 & -3.4169412359 \\
\hline C23 & -7.8361329083 & 1.5823252917 & -2.9979064457 \\
\hline C24 & -7.4674861372 & ๑. 8059474931 & -1.9621265499 \\
\hline $\mathrm{C} 25$ & -4.7760568777 & 1.0508457578 & -5.0790593923 \\
\hline C26 & -6.5948861115 & 2.7591867864 & ๑. 0498085242 \\
\hline $\mathrm{H} 27$ & 1.0750578969 & -3.4113282440 & -1.1274380950 \\
\hline H28 & -0.3478404646 & -4.5768224107 & -2.7815488910 \\
\hline H29 & -2.4833325288 & -3.5292191828 & -3.5382203304 \\
\hline H30 & -3.1610831343 & -1.3281022757 & -2.6118344512 \\
\hline H31 & 1.0996880988 & -1.7450427000 & $\odot .7709826570$ \\
\hline H32 & 1.5753761854 & $-\odot .6229170505$ & $-\odot .4975014853$ \\
\hline H33 & -7.6278480462 & 2.7697062574 & $\odot .4093470643$ \\
\hline H34 & -5.9147508611 & 2.9409713660 & ๑.8860543744 \\
\hline H35 & -6.4601021893 & 3.5490991971 & $-\odot .6904895649$ \\
\hline H36 & -5.9462362418 & ๑. . 0455643638 & 1. 4612423731 \\
\hline H37 & -6.4817980570 & -1.1067627105 & ๑. 2265988227 \\
\hline H38 & -7.6501476739 & ๑. 0385736773 & ๑. 9399167844 \\
\hline H39 & -3.5595890533 & 3.8410069831 & -5.2165701545 \\
\hline $\mathrm{H} 40$ & -3.1025152333 & 4.1754777787 & -3.5252571208 \\
\hline H41 & -2.3203360816 & 2.8522150122 & -4.4058876095 \\
\hline H42 & -4.9995349195 & 1.5988749654 & -5.9985013162 \\
\hline H43 & -3.8620309046 & ๑. 4662586712 & -5.2165749619 \\
\hline H44 & -5.5975322253 & ๑. 3654741792 & -4.8581591022 \\
\hline H45 & -5.8762956949 & 4.2788944481 & -4.0229550242 \\
\hline H46 & -8.0884673677 & 3.6447312561 & -3.5927480698 \\
\hline H47 & -7.9943385889 & -0.1373853834 & -1.8237448141 \\
\hline H48 & -8.6840497296 & 1.2344128713 & -3.5910054876 \\
\hline H49 & -1.1572348366 & 1.8830569469 & ๑. 4084519232 \\
\hline H50 & $-\odot .0843136991$ & 1.3965013885 & $-\odot .9061554997$ \\
\hline H51 & 1.8626426533 & ๑. 2622001038 & 2.0313930803 \\
\hline H52 & 1.7484535309 & 1.5117107023 & ๑. 7880153037 \\
\hline H53 & $\odot .3051271030$ & 1.4980282463 & 3.5199938354 \\
\hline H54 & ๑. 2302842093 & 3. 9084905412 & 3.2236390878 \\
\hline H55 & ๑.9770045133 & 3.7489566831 & 1.5383401675 \\
\hline H56 & -1.1005025875 & ๑. 2025889710 & 2.4076927269 \\
\hline H57 & -3.1962463836 & ๑. 7560319494 & ๑. 9361186068 \\
\hline
\end{tabular}




$\begin{array}{rrrr}\text { H58 } & -3.5499127228 & -0.8852964964 & 0.2262655164 \\ \text { H59 } & -2.1593725520 & 0.8408363348 & -1.7128756604\end{array}$

Energy components, in hartrees:
(A) Nuclear repulsion............
3017.12442176212
(E) Total one-electron terms.....
$-8413.57714275109$
(I) Total two-electron terms......
3653.73517996102
(J) Coulomb............... 3853.18964030659
(K) Exchange+Correlation....... $\quad-199.45446034558$
(L) Electronic energy........... -4759.84196279008
(N) Total energy............ -1742.71754102796

SCFE: SCF energy: DFT

-1742.71754102796 hartrees

HOMO energy: -0.32381

LUMO energy: -0.18533

Thermodynamic properties calculated assuming an ideal gas

In the table below, the units for temperature

are kelvins, the units for $\mathrm{U}, \mathrm{H}$, and $\mathrm{G}$ are

$\mathrm{kcal} / \mathrm{mol}$ and the units for $\mathrm{Cv}$ and $\mathrm{S}$ are $\mathrm{cal} /(\mathrm{mol} \mathrm{K})$

The zero point energy (ZPE): $311.975 \mathrm{kcal} / \mathrm{mol}$ is not included in $U, H$, or $G$ in the table below

$\mathrm{T}=298.15 \mathrm{~K}$

\begin{tabular}{rrrrrr} 
& \multicolumn{1}{c}{ U } & \multicolumn{1}{c}{ CV } & \multicolumn{1}{c}{ S } & \multicolumn{1}{c}{ H } & \multicolumn{1}{c}{ G } \\
trans. & 0.889 & 2.981 & 44.401 & 1.481 & -11.757 \\
rot. & 0.889 & 2.981 & 36.315 & 0.889 & -9.939 \\
vib. & 17.554 & 111.249 & 116.438 & 17.554 & -17.162 \\
elec. & 0.000 & 0.000 & 0.000 & 0.000 & 0.000 \\
total & 19.331 & 117.211 & 197.155 & 19.924 & -38.858
\end{tabular}

Total internal energy, Utot (SCFE + ZPE + U): $\quad-1742.189571$ hartrees Total enthalpy, Htot (Utot + pV): -1742.188626 hartrees Total Gibbs free energy, Gtot (Htot - $\left.T^{\star} S\right)$ : -1742.282301 hartrees

\section{Single point calculation at the B3LYP/LACV3P**++//B3LYP/LACVP** level}

Energy components, in hartrees:
(A) Nuclear repulsion............
3017.12442176212
(E) Total one-electron terms.....
$-8413.45827612003$
(I) Total two-electron terms.... 3653.33330286143
(J) Coulomb............. 3852.83552305788
(K) Exchange+Correlation....... -199.50222019646
(L) Electronic energy.......... -4760.12497325860
(N) Total energy............. $-1743.00055149649 \quad(A+L)$

SCFE: SCF energy: DFT $\quad-1743.00055149649$ hartrees

HOMO energy: $\quad-0.33367$

LUMO energy: $\quad-\odot .19287$

B3LYP/LACV3P**++//B3LYP/LACVP** Total Gibbs free energy, Gtot (Htot - $T^{*} S$ ): -1742.565311 hartrees 


\section{$\mathrm{M}^{+} 6^{+}$}

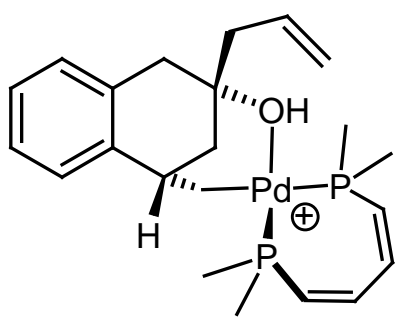

\section{NIMAG $\odot$}

\begin{tabular}{|c|c|c|c|}
\hline C1 & ๑.1926681978 & $\odot .5479731262$ & 1.1510606229 \\
\hline $\mathrm{C} 2$ & -0.7263463662 & 1.6367148888 & 0.5934616405 \\
\hline C3 & -0.0395497643 & 3. 0138710275 & ๑. 4694682736 \\
\hline C4 & ๑. 9161267587 & 3. 2971829636 & 1.6282448286 \\
\hline C5 & 2.0306903993 & 2.2482640139 & 1.6196705973 \\
\hline C6 & 1.4777239562 & 0.8330471653 & 1.6533329701 \\
\hline C7 & $-\odot .2452986883$ & $-\odot .7865234316$ & 1.1522882437 \\
\hline C8 & $\odot .5505319480$ & -1.8137767529 & 1.6554571585 \\
\hline C9 & 1.8154278574 & -1.5213282281 & 2.1746072837 \\
\hline C10 & 2.2690028634 & $-\odot .2046373417$ & 2.1699193460 \\
\hline C11 & -2.0857706019 & 1.7341595901 & 1. 3078261051 \\
\hline $\mathrm{Pd} 12$ & -2.0085658555 & 2.7177202154 & 3.1528607444 \\
\hline P13 & -4.1968986979 & 2.1274915966 & 3.5048417605 \\
\hline C14 & -5.2523125397 & 1.7358129757 & 2.0507889069 \\
\hline 015 & $\odot .1774058543$ & 3.1430579891 & 2.9069283567 \\
\hline C16 & 1.4796458913 & 4.7313014650 & 1.6114683146 \\
\hline C17 & ๑. 4421136183 & 5.8168091122 & 1.7310634583 \\
\hline C18 & ๑.2371484577 & 6.7774089346 & ๑. 8281787388 \\
\hline P19 & -1.8554082498 & 3.9098634863 & 5.3514019639 \\
\hline C20 & -2.9614603058 & 3.3855006243 & 6.7144619347 \\
\hline C21 & -4.3060630769 & 3. 3899860317 & 6.7080228283 \\
\hline C22 & -5.1971170172 & 3.8079522565 & 5.6113552549 \\
\hline C23 & -5.2143490624 & 3. 4000189870 & 4.3292923209 \\
\hline $\mathrm{C} 24$ & -2.1351172466 & 5.7354804271 & 5.2569045253 \\
\hline C25 & $-\odot .2002097288$ & 3. 7870865067 & 6.1572622987 \\
\hline C26 & -4.3004866298 & ๑. 6137423744 & 4.5475821675 \\
\hline $\mathrm{H} 27$ & -3.7865987786 & ๑. 7809590099 & 5.4954911156 \\
\hline H28 & -4.8173313363 & ๑. 9159716803 & 1.4758282378 \\
\hline $\mathrm{H} 29$ & -6.2519128221 & 1.4462208785 & 2.3873316855 \\
\hline H30 & -5.3300824721 & 2.6120056094 & 1.4011802386 \\
\hline H31 & -5.3450783573 & ๑. 3535100336 & 4.7404274154 \\
\hline H32 & -3.8082412793 & $-\odot .2080717696$ & 4.0211358424 \\
\hline H33 & -5.9552792312 & 3.8476558190 & 3.6684972110 \\
\hline H34 & -5.9704009430 & 4.5182612518 & 5.9088693783 \\
\hline H35 & -4.8165789630 & 3.1315412522 & 7.6363558748 \\
\hline H36 & -2.4575780804 & 3.0737915626 & 7.6288383245 \\
\hline H37 & -3.1334290355 & 5.9313112868 & 4.8615425374 \\
\hline H38 & -2.0374270549 & 6.2005278852 & 6.2427813090 \\
\hline H39 & -1.4012105030 & 6.1736716235 & 4.5769385419 \\
\hline $\mathrm{H} 40$ & $\odot .0056046175$ & 2.7471444276 & 6.4216378109 \\
\hline H41 & $\odot .5605205035$ & 4.1210929998 & 5.4492134617 \\
\hline $\mathrm{H} 42$ & -0.1468341338 & 4.4011092242 & 7.0611633440 \\
\hline H43 & -0.5125761005 & 7.5499247049 & ๑. 9761946208 \\
\hline H44 & $\odot .822596926 \odot$ & 6.8352926135 & $-\odot .0865767020$ \\
\hline H45 & $-\odot .1609274553$ & 5.7998287150 & 2.6374696482 \\
\hline H46 & 2.1972080920 & 4.8124751945 & 2.4402583869 \\
\hline $\mathrm{H} 47$ & 2.0482931771 & 4.8617415984 & $\odot .6829850049$ \\
\hline H48 & $\odot .5482943754$ & 2.3667045976 & 3. 3539916192 \\
\hline H49 & 2.6330299783 & 2.4009764563 & ๑. 7143536298 \\
\hline H50 & 2.7113160339 & 2.4162467172 & 2.4651512927 \\
\hline H51 & $\odot .5505501872$ & 3.0642064330 & -0.4531266435 \\
\hline H52 & $-\odot .7934713617$ & 3.8050965587 & ๑. 4035326951 \\
\hline H53 & -0.9837132997 & 1.3263099806 & -0.4325690005 \\
\hline H54 & -2.5093432014 & $\odot .7402768407$ & 1.4720317949 \\
\hline H55 & -2.7823363017 & 2.3192288152 & $\odot .6979000066$ \\
\hline H56 & -1.2213939272 & -1.0252392642 & ๑. 7361828021 \\
\hline H57 & $\odot .1890629704$ & -2.8376723602 & 1.6381950329 \\
\hline H58 & 2.4435952517 & -2.3126311751 & 2.5722001865 \\
\hline H59 & 3. 2565540309 & ๑. . 0281927155 & 2.5623826656 \\
\hline
\end{tabular}


Energy components, in hartrees:
(A) Nuclear repulsion...........
3138.00422918007
(E) Total one-electron terms.....
$-8655.13529614314$
(I) Total two-electron terms.....
3774.39679636719

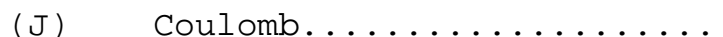
3973.85312031724
(K) Exchange+Correlation.......
$-199.45632395005$
(L) Electronic energy........... -4880.73849977595
(N) Total energy............. -1742.73427059588

SCFE: SCF energy: DFT $\quad-1742.73427059588$ hartrees

HOMO energy: $\quad-0.32604$

LUMO energy: $\quad-\odot .18367$

Thermodynamic properties calculated assuming an ideal gas

In the table below, the units for temperature

are kelvins, the units for $\mathrm{U}, \mathrm{H}$, and $\mathrm{G}$ are

$\mathrm{kcal} / \mathrm{mol}$ and the units for $\mathrm{Cv}$ and $\mathrm{S}$ are $\mathrm{cal} /(\mathrm{mol} \mathrm{K})$

The zero point energy (ZPE): $\quad 313.685 \mathrm{kcal} / \mathrm{mol}$

is not included in $U, H$, or $G$ in the table below

$\mathrm{T}=298.15 \mathrm{~K}$

\begin{tabular}{rrrrrr} 
& \multicolumn{1}{c}{ U } & \multicolumn{1}{c}{ CV } & \multicolumn{1}{c}{ S } & \multicolumn{1}{c}{$\mathrm{H}$} & \multicolumn{1}{c}{ G } \\
trans. & 0.889 & 2.981 & 44.401 & 1.481 & -11.757 \\
rot. & 0.889 & 2.981 & 35.899 & 0.889 & -9.815 \\
vib. & 17.297 & 110.672 & 113.034 & 17.297 & -16.404 \\
elec. & 0.000 & 0.000 & 0.000 & 0.000 & 0.000 \\
total & 19.074 & 116.633 & 193.334 & 19.667 & -37.976
\end{tabular}

Total internal energy, Utot (SCFE + ZPE + U): -1742.203984 hartrees Total enthalpy, Htot (Utot $+\mathrm{pV}):-1742.203040$ hartrees Total Gibbs free energy, Gtot (Htot - $\left.T^{\star} S\right)$ : -1742.294899 hartrees

\section{Single point calculation at the B3LYP/LACV3P**++//B3LYP/LACVP** level}

Energy components, in hartrees:
(A) Nuclear repulsion......... 3138.00422918007
(E) Total one-electron terms.... -8655.08940992883
(I) Total two-electron terms.... 3774.07254375621
(J) Coulomb............. 3973.58962094590
(K) Exchange+Correlation....... -199.51707718970
(L) Electronic energy.......... $-4881.01686617262 \quad$ (E+I)
(N) Total energy............ $-1743.01263699254 \quad(\mathrm{~A}+\mathrm{L})$

SCFE: SCF energy: DFT $\quad-1743.01263699254$ hartrees

HOMO energy: $\quad-\odot .33467$

LUMO energy: $-\odot .19162$

B3LYP/LACV3P**++//B3LYP/LACVP** Total Gibbs free energy, Gtot (Htot - T*S): -1742.573265 hartrees 
${\mathrm{M} 17^{+}}^{+}$

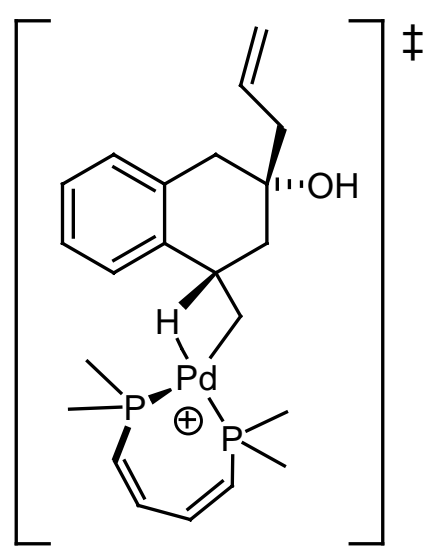

\section{NIMAG 1}

\begin{tabular}{|c|c|c|c|}
\hline C1 & -1.3222586411 & ๑. 3524295289 & -0.7881450336 \\
\hline $\mathrm{C} 2$ & -0.9256475873 & -0.9724730573 & -1.3548836692 \\
\hline C3 & -0.0515284847 & -1.7945203839 & -0.6160663045 \\
\hline C4 & $\odot .5519594276$ & -1.3497267910 & ๑. 7037061653 \\
\hline C5 & -0.1910882310 & -0.1835273512 & 1.3652703093 \\
\hline C6 & -0.4130752628 & $\odot .9145223317$ & ๑. 2919746972 \\
\hline C7 & ๑. 2501792514 & -3.0702007240 & -1.1146626027 \\
\hline C8 & $-\odot .2778569528$ & -3.5221879842 & -2.3213151045 \\
\hline C9 & -1.1413293740 & -2.7000096197 & -3.0544328254 \\
\hline C10 & -1.4575209537 & -1.4360920799 & -2.5699606364 \\
\hline 011 & -1.4413877564 & -0.7170659219 & 1.7997899845 \\
\hline C12 & 0.6178234286 & ๑. 3819348822 & 2.5617219986 \\
\hline C13 & -0.1577664605 & 1.3533815599 & 3.4133320235 \\
\hline C14 & $\odot .1029886020$ & 2.6585975999 & 3.5184917001 \\
\hline C15 & -2.6801207149 & ๑. 7549953153 & -0.8147364054 \\
\hline Pd16 & -2.0866288522 & 1.9908733097 & -2.5185814925 \\
\hline P17 & -4.2743221978 & 2.8948226457 & -2.9736257659 \\
\hline C18 & -4.6857932110 & 3. 1190612868 & -4.7435932934 \\
\hline C19 & -4.1064371477 & 3.9433167420 & -5.6350014758 \\
\hline C2O & -2.9915802624 & 4.8897041979 & -5.4349768196 \\
\hline C21 & -1.7634457920 & 4.6632402458 & -4.9341746746 \\
\hline P22 & -1.0429094039 & 3.0759326336 & -4.3756675359 \\
\hline C23 & $\odot .7322852295$ & $3.51276400 \odot 2$ & -4.1774321098 \\
\hline $\mathrm{C} 24$ & -1.0725201680 & 1.9994582589 & -5.8725556816 \\
\hline $\mathrm{C} 25$ & -4.5380081735 & 4.5584099550 & -2.2148199886 \\
\hline $\mathrm{C} 26$ & -5.7251223387 & 1.9206973709 & -2.3839146670 \\
\hline $\mathrm{H} 27$ & $\odot .9 \odot 87260342$ & -3.7157984194 & -0.5393441261 \\
\hline $\mathrm{H} 28$ & $-\odot .0221215502$ & -4.5115067261 & -2.6884523078 \\
\hline $\mathrm{H} 29$ & -1.5568599296 & -3.0420481615 & -3.9974416802 \\
\hline H30 & -2.1149787018 & $-\odot .7845142761$ & -3.1418148677 \\
\hline H31 & $\odot .5648166759$ & -2.1925287467 & 1.4028824913 \\
\hline H32 & 1.5979366716 & -1.0535828267 & 0.5452181587 \\
\hline H33 & -5.5424942952 & 4.9309330530 & -2.4343074561 \\
\hline H34 & -4.4091619590 & 4.4787963268 & -1.1318639643 \\
\hline H35 & -3.7966923532 & 5.2592211569 & -2.6031346932 \\
\hline H36 & -5.6465122476 & 1.7681627443 & -1.3045562669 \\
\hline H37 & -5.7311528343 & $\odot .9391980299$ & -2.8660333712 \\
\hline H38 & -6.6654506915 & 2.4343906504 & -2.6045059549 \\
\hline H39 & 1.1269682921 & 3. 9870670224 & -5.0809286690 \\
\hline $\mathrm{H} 40$ & ๑. 8503241636 & 4.1928472722 & -3.3299083245 \\
\hline H41 & 1.2988124264 & 2.6023064184 & -3.9684401001 \\
\hline $\mathrm{H} 42$ & -0.5776181719 & 2.4914230147 & -6.7143182975 \\
\hline $\mathrm{H} 43$ & -0.5619529717 & 1.0602919679 & -5.6442230644 \\
\hline $\mathrm{H} 44$ & -2.1079794961 & 1.7785089994 & -6.1398340340 \\
\hline H45 & -1.0774410949 & 5.5078130988 & -4.8825654443 \\
\hline H46 & -3.1946754495 & 5.8965005242 & -5.8053020284 \\
\hline $\mathrm{H} 47$ & -5.5259694726 & 2.5167099382 & -5.0878721983 \\
\hline H48 & -4.5542216896 & 3.9869060823 & -6.6295352073 \\
\hline H49 & $-\odot .8571654333$ & 1.8082415949 & 0.7411366074 \\
\hline H50 & $\odot .5627913253$ & 1.1974092805 & -0.1147690226 \\
\hline H51 & ๑.9222542792 & -0.4741924582 & 3.1780100152 \\
\hline H52 & 1.5313480030 & 0.8578606443 & 2.1869993313 \\
\hline H53 & -0.9778306052 & $\odot .9346252556$ & 3.9986403086 \\
\hline H54 & -0.4832956953 & 3. 3080062631 & 4.1621458666 \\
\hline H55 & $\odot .9294625201$ & 3. 1199139562 & 2.9819064124 \\
\hline
\end{tabular}




$\begin{array}{rrrr}\text { H56 } & -1.9229642491 & -0.0215981968 & 2.2682730828 \\ \text { H57 } & -3.0143379129 & 1.4728958845 & -0.0691395403 \\ \text { H58 } & -3.4291330241 & 0.0550624362 & -1.1727872817 \\ \text { H59 } & -0.6915561794 & 1.3300004917 & -2.0680246763\end{array}$

Energy components, in hartrees:
(A) Nuclear repulsion...........
3012.72562600915
(E) Total one-electron terms.....
$-8404.65459106548$
(I) Total two-electron terms.....
3649.22172508237

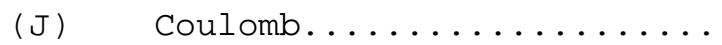
3848.63086813990
(K) Exchange+Correlation.......
$-199.40914305753$
(L) Electronic energy.......... -4755.43286598312
(N) Total energy............. -1742.70723997396

SCFE: SCF energy: DFT $\quad-1742.70723997396$ hartrees

HOMO energy: $\quad-0.32930$

LUMO energy: $\quad-\odot .17630$

Thermodynamic properties calculated assuming an ideal gas

In the table below, the units for temperature are kelvins, the units for $\mathrm{U}, \mathrm{H}$, and $\mathrm{G}$ are

$\mathrm{kcal} / \mathrm{mol}$ and the units for $\mathrm{Cv}$ and $\mathrm{S}$ are $\mathrm{cal} /(\mathrm{mol} \mathrm{K})$

The zero point energy (ZPE): $309.689 \mathrm{kcal} / \mathrm{mol}$

is not included in $U, H$, or $G$ in the table below

$T=298.15 \mathrm{~K}$

\begin{tabular}{rrrrrr} 
& \multicolumn{1}{c}{ U } & \multicolumn{1}{c}{ CV } & \multicolumn{1}{c}{ S } & \multicolumn{1}{c}{ H } & \multicolumn{1}{c}{ G } \\
trans. & 0.889 & 2.981 & 44.401 & 1.481 & -11.757 \\
rot. & 0.889 & 2.981 & 36.322 & 0.889 & -9.941 \\
vib. & 17.549 & 111.383 & 116.618 & 17.549 & -17.220 \\
elec. & 0.000 & 0.000 & 0.000 & 0.000 & 0.000 \\
total & 19.327 & 117.345 & 197.340 & 19.919 & -38.918
\end{tabular}

Total internal energy, Utot (SCFE + ZPE + U): $\quad-1742.182921$ hartrees Total enthalpy, Htot (Utot + pV): $\quad-1742.181976$ hartrees Total Gibbs free energy, Gtot (Htot - $T^{\star} \mathrm{S}$ ): $\quad-1742.275739$ hartrees

\section{Single point calculation at the B3LYP/LACV3P**++//B3LYP/LACVP** level}

Energy components, in hartrees:
(A) Nuclear repulsion.......... 3012.72562600915
(E) Total one-electron terms ....
(I) Total two-electron terms..... 3648.85732959543
(J) Coulomb............. 3848.31746903253
(K) Exchange+Correlation....... -199.46013943711
(L) Electronic energy.......... -4755.71698062422
(N) Total energy............ -1742.99135461507
$(E+I)$
$(A+L)$

SCFE: SCF energy: DFT -1742.99135461507 hartrees

$\begin{array}{ll}\text { HOMO energy: } & -0.33833 \\ \text { LUMO energy: } & -0.18552\end{array}$

B3LYP/LACV3P**++//B3LYP/LACVP** Total Gibbs free energy, Gtot (Htot - $\left.{ }^{*} \mathrm{~S}\right)$ : -1742.559854 hartrees 


\section{$\mathrm{M}^{+} 8^{+}$}

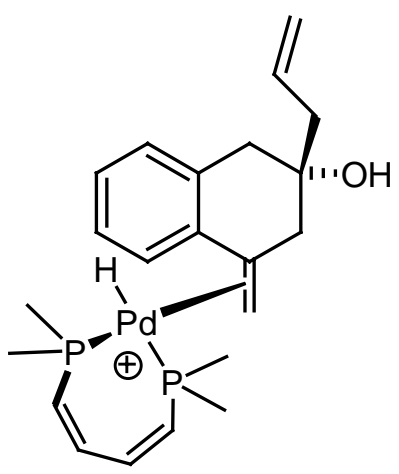

\section{NIMAG $\odot$}

$-1.4932765058$

$-0.9889120244$

$-1.7368320992$

$-2.9717107357$

$-3.4586365507$

$-2.7115893962$

$-1.2535679097$

$-0.1485787720$

0.9137088649

$\odot .2729903528$

0.7494548460

2. 0872271876

3. 2732493313

2. 2489310637

$-0.7847564588$

0.4847604033

1. 3842052534

2. 7119405446

2. 7961195298

4.3834321535

1. 6642107530

3. 0782222870

3. 9951171498

5. 0070825363

4. 8331189914

3. 8387584612

$-3.5579769462$

$-4.4159667848$

$-3.0803870883$

$-0.9012013303$

$-2.0951914148$

$-0.8799660115$

4.6889241720

4. 2472920778

5.1658838590

1. 4827121272

0.7047828037

2. 0957781648

4. 3864115790

4. 4833792749

2. 9673970139

2. 8120792424

1. 3512452193

1.9503385079

5.7182682127

6. 0226538560

2. 4270754150

4. 0632222744

1.7634370578

1.2882508452

$-0.3414588013$

1.0397424039

$\odot .8909649204$

3. 3085624733

3. 2474459650

$-0.1233498281$

1.5205618470

$\odot .1177245538$
$-2.4498307942$

$-1.2842584805$

- 0.6608526200

$-1.2139492092$

$-2.3666210072$

$-2.9926895401$

$\odot .5924760982$

1. 3457830237

$\odot .3153637575$

$-0.6586583754$

$-0.7793024382$

$-2.5594999564$

$-4.3853306277$

$-5.9144698743$

1.9405970870

2. 4283299902

3. 3927811712

3. 4488389349

$-2.8445906726$

$-1.9761950019$

$-2.2487904026$

$-4.5770710110$

$-5.4421395832$

$-5.2186169377$

- 4.7874329181

$-4.2938610432$

$-0.7246814454$

$-2.7781241457$

$-3.8968193150$

$-2.9464777674$

1. 2765849102

๑. 3231336900

$-2.1502638828$

$-0.9032291323$

$-2.3315989423$

$-1.1785720406$

$-2.7692966381$

$-2.4176384686$

$-5.1981967677$

$-3.4211285206$

$-4.1761796625$

$-6.7705224007$

$-5.7632935735$

$-6.1078800721$

$-4.6907596546$

$-5.4949417253$

$-4.9170031883$

- 6.4140411047

$\odot .8221227111$

- 0.2101100805

2. 9846872539

1. 9365230412

4. 1049588697

4.1761469334

2. 7847966695

2. 4531218775

- 0.0888673658

$-1.2097272254$ 
$\begin{array}{llll}\text { H59 } & -0.7875735929 & 1.7326389537 & -2.4384376344\end{array}$

Energy components, in hartrees:
(A) Nuclear repulsion...........
2992.44427579830
(E) Total one-electron terms.... - 8364.20597298286
(I) Total two-electron terms..... 3629.05033187552
(J) Coulomb................ 3828.45427543202
(K) Exchange+Correlation...... $\quad-199.40394355650$
(L) Electronic energy. . . . . . . . . -4735.15564110734
(N) Total energy............. -1742.71136530905
$(E+I)$
$(A+L)$

SCFE: SCF energy: DFT $\quad-1742.71136530905$ hartrees

HOMO energy: $\quad-0.32763$

LUMO energy: $-\odot .18382$

Thermodynamic properties calculated assuming an ideal gas

In the table below, the units for temperature are kelvins, the units for $U, H$, and $G$ are $\mathrm{kcal} / \mathrm{mol}$ and the units for $\mathrm{Cv}$ and $\mathrm{S}$ are $\mathrm{cal} /(\mathrm{mol} \mathrm{K})$

The zero point energy (ZPE): $310.414 \mathrm{kcal} / \mathrm{mol}$ is not included in $U, H$, or $G$ in the table below

$\mathrm{T}=298.15 \mathrm{~K}$

\begin{tabular}{rrrrrr} 
& \multicolumn{1}{c}{ U } & \multicolumn{1}{c}{ CV } & \multicolumn{1}{c}{ S } & \multicolumn{1}{c}{ H } & \multicolumn{1}{c}{ G } \\
trans. & 0.889 & 2.981 & 44.401 & 1.481 & -11.757 \\
rot. & 0.889 & 2.981 & 36.386 & 0.889 & -9.960 \\
vib. & 17.349 & 110.939 & 113.841 & 17.349 & -16.592 \\
elec. & $0.00 \odot$ & $0.00 \odot$ & $0.00 \odot$ & 0.000 & 0.000 \\
total & 19.127 & 116.901 & 194.628 & 19.719 & -38.309
\end{tabular}

Total internal energy, Utot (SCFE + ZPE + U): -1742.186208 hartrees Total enthalpy, Htot (Utot + pV): -1742.185264 hartrees Total Gibbs free energy, Gtot (Htot - $\mathrm{T}^{\star} \mathrm{S}$ ): -1742.277738 hartrees

\section{Single point calculation at the B3LYP/LACV3P**++//B3LYP/LACVP** level}

Energy components, in hartrees:

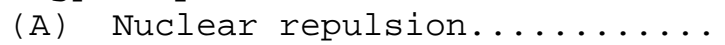
2992.44427579830
(E) Total one-electron terms.... -8364.10760705387
(I) Total two-electron terms.... 3628.66783961226
(J) Coulomb.............. 3828.12303439426
(K) Exchange+Correlation . . . . . . $\quad-199.45519478200$
(L) Electronic energy......... -4735.43976744161 (E+I)
(N) Total energy............ $-1742.99549164331 \quad(\mathrm{~A}+\mathrm{L})$

SCFE: SCF energy: DFT $\quad-1742.99549164331$ hartrees

HOMO energy: $\quad-0.33634$

LUMO energy: -0.19172

B3LYP/LACV3P**++//B3LYP/LACVP** Total Gibbs free energy, Gtot (Htot - T*S): -1742.561865 hartrees 


\section{$\mathrm{M}^{+} 9^{+}$}<smiles>[BH3-][P@]1(C)C=CC=CP1(C)(C)C</smiles>

(NIMAG $1,7 \mathrm{~cm}-1$ )

$\begin{array}{lrrr}\text { C1 } & 4.2546305225 & -0.6403675473 & -2.6140402482 \\ \text { P2 } & 3.1363895508 & 0.650050 \odot 242 & -1.9438259633 \\ \text { C3 } & 3.0392673421 & 2.0178305220 & -3.1334045722 \\ \text { C4 } & 2.7601684713 & 1.9751967603 & -4.4492461235 \\ \text { C5 } & 2.4162215256 & 0.8347709441 & -5.3171612301 \\ \text { C6 } & 1.4023448794 & -0.0407934109 & -5.2071348902 \\ \text { P7 } & 0.1096553567 & -0.1087398485 & -3.9078385833 \\ \text { C8 } & -1.0155776217 & 1.2968513231 & -4.3254068076 \\ \text { C9 } & 4.0159579865 & 1.3914391083 & -0.5191281216 \\ \text { Pd10 } & 1.0775351906 & -0.1825003468 & -1.6234856088 \\ \text { C11 } & -0.8484542625 & -1.6033494837 & -4.4095487529 \\ \text { H12 } & 1.7446165275 & -0.2722814497 & -0.2152367712 \\ \text { H13 } & 1.2926701440 & -0.7806790353 & -5.9996264063 \\ \text { H14 } & 3.0275141201 & 0.7731863086 & -6.2194209318 \\ \text { H15 } & 3.2631417627 & 2.9871003364 & -2.6902485587 \\ \text { H16 } & 2.8519749231 & 2.9202280963 & -4.9887175834 \\ \text { H17 } & 4.9658835450 & 1.8221560972 & -0.8505668040 \\ \text { H18 } & 3.3973445530 & 2.1704886664 & -0.0675080397 \\ \text { H19 } & 4.2040195743 & 0.6198391844 & 0.2305021855 \\ \text { H20 } & 5.2375517040 & -0.2022287666 & -2.8130892560 \\ \text { H21 } & 4.3526296848 & -1.4428390304 & -1.8789002971 \\ \text { H22 } & 3.8424182731 & -1.0486718194 & -3.5377543914 \\ \text { H23 } & -1.3292918547 & 1.2495713062 & -5.3718305394 \\ \text { H24 } & -1.8986150144 & 1.2515152113 & -3.6815421373 \\ \text { H25 } & -0.5026272490 & 2.2442275632 & -4.1441933392 \\ \text { H26 } & -1.1802613948 & -1.5281577188 & -5.4494903069 \\ \text { H27 } & -0.2322404794 & -2.4986337534 & -4.2916789175 \\ \text { H28 } & -1.7264547440 & -1.7047174539 & -3.7660984553\end{array}$

Energy components, in hartrees:
(A) Nuclear repulsion............
1007.82352412010
(E) Total one-electron terms.....
$-3580.51720637419$
(I) Total two-electron terms.....
1448.34634054520

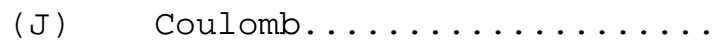
1557.09480173400
(K) Exchange+Correlation.......
$-108.74846118880$
(L) Electronic energy.......... -2132.17086582899
(N) Total energy............ -1124.34734170889

SCFE: SCF energy: DFT

-1124.34734170889 hartrees

$\begin{array}{ll}\text { HOMO energy: } & -0.37113 \\ \text { LUMO energy: } & -0.23282\end{array}$

Thermodynamic properties calculated assuming an ideal gas

In the table below, the units for temperature are kelvins, the units for $\mathrm{U}, \mathrm{H}$, and $\mathrm{G}$ are $\mathrm{kcal} / \mathrm{mol}$ and the units for $\mathrm{CV}$ and $\mathrm{S}$ are $\mathrm{cal} /(\mathrm{mol} \mathrm{K})$

The zero point energy (ZPE): $143.800 \mathrm{kcal} / \mathrm{mol}$ is not included in $\mathrm{U}, \mathrm{H}$, or $\mathrm{G}$ in the table below 
$\mathrm{T}=298.15 \mathrm{~K}$

\begin{tabular}{rrrrrr} 
& \multicolumn{1}{c}{ U } & \multicolumn{1}{c}{ CV } & \multicolumn{1}{c}{ S } & \multicolumn{1}{c}{ H } & \multicolumn{1}{c}{ G } \\
trans. & 0.889 & 2.981 & 42.798 & 1.481 & -11.279 \\
rot. & 0.889 & 2.981 & 31.958 & 0.889 & -8.640 \\
vib. & 7.878 & 51.312 & 47.143 & 7.878 & -6.178 \\
elec. & $0.00 \odot$ & $0.0 \odot \odot$ & $0.0 \odot \odot$ & $0.0 \odot \odot$ & $0.0 \odot \odot$ \\
total & 9.655 & 57.274 & 121.899 & 10.248 & -26.097
\end{tabular}

Total internal energy, Utot (SCFE + ZPE + U): -1124.102796 hartrees Total enthalpy, Htot (Utot + pV): -1124.101852 hartrees Total Gibbs free energy, Gtot (Htot - $T^{\star} \mathrm{S}$ ): -1124.159770 hartrees

\section{Single point calculation at the B3LYP/LACV3P**++//B3LYP/LACVP** level}

Energy components, in hartrees:
(A) Nuclear repulsion.......... 1007.82352412263
(E) Total one-electron terms.... - 3580.84372369125
(I) Total two-electron terms ..... 1448.53992005244
(J) Coulomb.............. 1557.32412171736
(K) Exchange+Correlation...... $\quad-108.78420166493$
(L) Electronic energy.......... -2132.30380363882 (E+I)
(N) Total energy ............ -1124.48027951618 (A+L)

SCFE: SCF energy: DFT $\quad-1124.48027951618$ hartrees

HOMO energy: $\quad-0.37557$

LUMO energy: $\quad-0.23591$

B3LYP/LACV3P**++//B3LYP/LACVP** Total Gibbs free energy, Gtot (Htot - $\mathrm{T}^{*} \mathrm{~S}$ ) : -1124.292708 hartrees

B3LYP/LACV3P**++//B3LYP/LACVP** Total Gibbs free energy, Gtot (Htot - $T^{*} S$ ) of $\mathrm{M}^{+}$and product: -1742.552790 hartrees<smiles></smiles>

(NIMAG 1, -27 cm-1)

$\begin{array}{lrrr}\text { C1 } & 4.2907762006 & -0.4690781547 & -2.5738536296 \\ \text { P2 } & 3.0312875186 & 0.7324050307 & -1.9614829817 \\ \text { C3 } & 3.8584224883 & 1.5250069894 & -0.5211957504 \\ \text { Pd4 } & 1.0199771024 & -0.2178495573 & -1.5718313248 \\ \text { P5 } & 0.2017194502 & 0.0206902959 & -3.9126601277 \\ \text { C6 } & 1.4901449263 & 0.0494466796 & -5.2243224436 \\ \text { C7 } & 2.5088634940 & 0.9167532540 & -5.3596505899 \\ \text { C8 } & 2.8530309548 & 2.0644233537 & -4.4995322698 \\ \text { C9 } & 3.0620098186 & 2.1068379719 & -3.1723105635 \\ \text { 010 } & -0.7090905362 & -1.3028737992 & -0.9209136389 \\ \text { S11 } & -2.0586057400 & -0.5774706205 & -1.0051600676 \\ \text { 012 } & -2.9996998063 & -1.2829001879 & -1.8984326857 \\ \text { 013 } & -1.8664205388 & -.8667743539 & -1.2783327364 \\ \text { C14 } & -2.7140370151 & -0.7267037181 & 0.6672943953 \\ \text { C15 } & -0.8946870235 & -1.3349697721 & -4.5080777981 \\ \text { C16 } & -0.7771304405 & 1.5533694824 & -4.2427581466 \\ \text { H17 } & 1.5669396741 & -\odot .3488845934 & -0.1160791530 \\ \text { H18 } & -2.0229323096 & -0.2348267274 & 1.3508481073 \\ \text { H19 } & -3.6900609094 & -\odot .2428825463 & 0.6901966575 \\ \text { H20 } & -2.8137215207 & -1.7832873434 & 0.9134845554 \\ \text { H21 } & 1.3867260681 & -0.7193753913 & -5.9907147521 \\ \text { H22 } & 3.1316393368 & -.8277442279 & -6.2528248354\end{array}$




$\begin{array}{lrrr}\text { H23 } & 3.30 \odot 4479816 & 3.0754495728 & -2.7325813807 \\ \text { H24 } & 2.9994174899 & 2.9997334429 & -5.0463126165 \\ \text { H25 } & 4.8150238309 & 1.9699858456 & -0.8081171926 \\ \text { H26 } & 3.2040087001 & 2.2954884511 & -0.1100617881 \\ \text { H27 } & 4.0181132639 & 0.7700778523 & -.2520438963 \\ \text { H28 } & 5.2554077411 & 0.0215230196 & -2.7313926583 \\ \text { H29 } & 4.3979205807 & -1.2676342546 & -1.8346743372 \\ \text { H30 } & 3.9477662371 & -0.9042261759 & -3.5139360415 \\ \text { H31 } & -1.1526157942 & 1.5697693436 & -5.2699188662 \\ \text { H32 } & -1.6013066304 & 1.5672404888 & -3.5233148884 \\ \text { H33 } & -0.1464895050 & 2.4280863290 & -4.0663968142 \\ \text { H34 } & -1.2282800536 & -1.1481936729 & -5.5321593513 \\ \text { H35 } & -0.3532526716 & -2.2834428870 & -4.4675562561 \\ \text { H36 } & -1.7589366700 & -1.4077163965 & -3.8391427897\end{array}$

Energy components, in hartrees:
(A) Nuclear repulsion............
1899.83003900671
(E) Total one-electron terms.....
$-6292.35573224782$
(I) Total two-electron terms.....
2604.22385373091
2771.39384690502

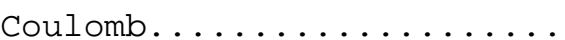
$\begin{array}{lrr}\text { (K) } & \text { Exchange+Correlation........ } & -167.16999317410 \\ \text { (L) Electronic energy........... } & -3688.13187851690\end{array}$
(N) Total energy............ -1788.30183951019

SCFE: SCF energy: DFT

-1788.30183951019 hartrees

HOMO energy: $\quad-0.21783$

LUMO energy: $\quad-0.07658$

Thermodynamic properties calculated assuming an ideal gas

In the table below, the units for temperature

are kelvins, the units for $\mathrm{U}, \mathrm{H}$, and $\mathrm{G}$ are

$\mathrm{kcal} / \mathrm{mol}$ and the units for $\mathrm{Cv}$ and $\mathrm{S}$ are $\mathrm{cal} /(\mathrm{mol} \mathrm{K})$

The zero point energy (ZPE): $176.600 \mathrm{kcal} / \mathrm{mol}$

is not included in $\mathrm{U}, \mathrm{H}$, or $\mathrm{G}$ in the table below

$\mathrm{T}=298.15 \mathrm{~K}$

\begin{tabular}{rrrrrr} 
& \multicolumn{1}{c}{ U } & \multicolumn{1}{c}{ CV } & \multicolumn{1}{c}{ S } & \multicolumn{1}{c}{ H } & \multicolumn{1}{c}{ G } \\
trans. & 0.889 & 2.981 & 43.666 & 1.481 & -11.538 \\
rot. & 0.889 & 2.981 & 33.855 & 0.889 & -9.205 \\
vib. & 11.977 & 73.975 & 77.490 & 11.977 & -11.127 \\
elec. & 0.000 & 0.000 & 0.000 & 0.000 & 0.000 \\
total & 13.754 & 79.936 & 155.011 & 14.347 & -31.870
\end{tabular}

Total internal energy, Utot (SCFE + ZPE + U): $\quad-1787.998491$ hartrees Total enthalpy, Htot (Utot + pV): $\quad-1787.997547$ hartrees

Total Gibbs free energy, Gtot (Htot - $\left.T^{\star} \mathrm{S}\right): \quad-1788.071197$ hartrees

\section{Single point calculation at the B3LYP/LACV3P**++//B3LYP/LACVP** level}

Energy components, in hartrees:
(A) Nuclear repulsion...........
1899.83003900709
(E) Total one-electron terms.....
$-6292.22993857304$
(I) Total two-electron terms.....
2603.84708562023
(J) Coulomb..............
2771.07441950392
(K) Exchange+Correlation......
$-167.22733388369$
(L) Electronic energy...........
$-3688.38285295281$
$-1788.55281394572$ 
SCFE: SCF energy: DFT -1788.55281394572 hartrees

HOMO energy: $\quad-0.22960$

LUMO energy: $\quad-0.08789$

B3LYP/LACV3P**++//B3LYP/LACVP** Total Gibbs free energy, Gtot (Htot - $\mathrm{T}^{*} \mathrm{~S}$ ): -1788.322171 hartrees

\section{Educt}<smiles></smiles>

\section{NIMAG $\odot$}

\begin{tabular}{|c|c|c|c|}
\hline C1 & $1.588600000 \odot$ & $-1.945900000 \odot$ & -0.3106000000 \\
\hline C2 & $\odot .4729000000$ & -2.7620000000 & -0.4804000000 \\
\hline $\mathrm{C} 3$ & -0.8108000000 & -2.2271000000 & $-0.366500000 \odot$ \\
\hline $\mathrm{C} 4$ & -0.9649000000 & -0.8690000000 & -0.1048000000 \\
\hline C5 & $\odot .166200 \odot \odot \odot \odot$ & $-\odot . \odot 748 \odot \odot \odot \odot \odot \odot$ & ๑. . \\
\hline C6 & 1. $4710 \odot \odot \odot \odot \odot \odot$ & $-\odot .5804 \odot \odot \odot \odot \odot \odot$ & $-\odot . \odot \odot 64 \odot \odot \odot \odot \odot \odot$ \\
\hline 07 & $-\odot .025900000 \odot$ & 1.3227000000 & $\odot .188100000 \odot$ \\
\hline S8 & $-\odot .479200000 \odot$ & 1.983600०००० & 1.6360000000 \\
\hline 09 & $-1.3750 \odot \odot \odot \odot \odot \odot$ & 1. $.8110 \odot \odot \odot \odot \odot$ & 2. $34670 \odot \odot \odot \odot \odot$ \\
\hline $\mathrm{C} 10$ & 2.6963000000 & ๑. 2831000000 & $\odot .1860000000$ \\
\hline C11 & 3. 3951000000 & $\odot .1851000000$ & 1.5835000000 \\
\hline $\mathrm{C} 12$ & 4. 4138000000 & 1.3595000000 & 1.6690000000 \\
\hline $\mathrm{C} 13$ & 5.2195000000 & 1.3890000000 & 2.9374000000 \\
\hline C14 & $6.54660 \odot \odot \odot \odot \odot$ & 1. $50870 \odot \odot \odot \odot \odot$ & 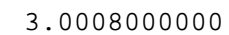 \\
\hline 015 & $\odot .7198000000$ & 2.5099000000 & 2. 3015000000 \\
\hline C16 & -1.3858000000 & 3.3715000000 & ๑. 9578000000 \\
\hline 017 & 2.4552000000 & $\odot .282700000 \odot$ & 2.6449000000 \\
\hline C18 & $4.0683000 \odot \odot \odot$ & -1.1869000000 & 1. $82860 \odot \odot \odot \odot \odot$ \\
\hline C19 & $5.1620 \odot \odot \odot \odot \odot \odot$ & -1.5796000000 & $\odot .875800 \odot \odot \odot \odot$ \\
\hline C20 & $5.26370000 \odot \odot$ & -2.7627000000 & ๑. $26480000 \odot \odot$ \\
\hline $\mathrm{H} 21$ & -1.6925000000 & 3.9872000000 & 1.8047000000 \\
\hline $\mathrm{H} 22$ & $-\odot .7231000 \odot \odot \odot$ & 3. $928800000 \odot$ & ๑. $296000 \odot \odot \odot \odot$ \\
\hline H23 & -2.2536000000 & 2.9898000000 & $\odot .4205000000$ \\
\hline $\mathrm{H} 24$ & 2.5832000000 & $-2.363400000 \odot$ & $-0.427800000 \odot$ \\
\hline H25 & ๑. 6061000000 & -3.8152000000 & -0.7105000000 \\
\hline H26 & -1.6859000000 & -2.8561000000 & $-\odot .4976000000$ \\
\hline $\mathrm{H} 27$ & -1.9475000000 & -0.4166000000 & $-0.035200000 \odot$ \\
\hline H28 & 3.4373000000 & $\odot .0234000000$ & -0.5788000000 \\
\hline H29 & 2.4237000000 & 1.3299000000 & $\odot .0217000000$ \\
\hline H30 & 2. . & 1. $1458000 \odot \odot \odot$ & 2. $61050 \odot \odot \odot \odot \odot$ \\
\hline H31 & 5.0806000000 & 1. 3518000000 & $\odot .7981000000$ \\
\hline H32 & 3. $8241000 \odot \odot \odot$ & $2.286100000 \odot$ & $1.596600 \odot \odot \odot \odot$ \\
\hline H33 & 4.4822000000 & -1.1369000000 & 2.8440000000 \\
\hline H34 & 3. $286600 \odot \odot \odot \odot$ & -1.9522000000 & 1.8546000000 \\
\hline H35 & 4.6406000000 & 1.3109000000 & 3.8567000000 \\
\hline H36 & 7.0720000000 & 1.5384000000 & 3. 9511000000 \\
\hline H37 & $7.15940 \odot \odot \odot \odot \odot$ & $1.589400 \odot \odot \odot \odot$ & $2.1052000 \odot \odot \odot$ \\
\hline H38 & 5.9512000000 & $-\odot .8449000000$ & $\odot .715400000 \odot$ \\
\hline H39 & 6.1027000000 & - 3.0011000000 & -0.3826000000 \\
\hline $\mathrm{H} 40$ & 4.5232000000 & -3.5468000000 & ๑. 4081000000 \\
\hline
\end{tabular}

Energy components, in hartrees:
(A) Nuclear repulsion...........
1816.07983302577
(E) Total one-electron terms.....
(I) Total two-electron terms.....

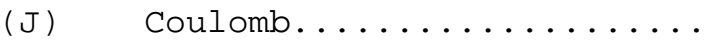
(K) Exchange+Correlation......
(L) Electronic energy.......... 


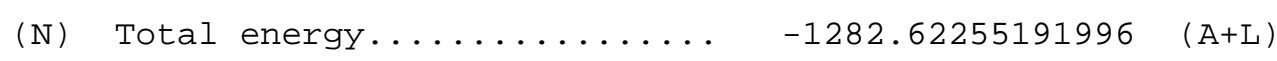

SCFE: SCF energy: DFT(b3lyp) $\quad-1282.62255191996$ hartrees

HOMO energy: $-\odot .23894$

LUMO energy: -0.01479

Thermodynamic properties calculated assuming an ideal gas

In the table below, the units for temperature

are kelvins, the units for $\mathrm{U}, \mathrm{H}$, and $\mathrm{G}$ are

$\mathrm{kcal} / \mathrm{mol}$ and the units for $\mathrm{Cv}$ and $\mathrm{S}$ are $\mathrm{cal} /(\mathrm{mol} \mathrm{K})$

The zero point energy (ZPE): $205.597 \mathrm{kcal} / \mathrm{mol}$

is not included in $\mathrm{U}, \mathrm{H}$, or $\mathrm{G}$ in the table below

$\mathrm{T}=298.15 \mathrm{~K}$

\begin{tabular}{rrrrrr} 
& \multicolumn{1}{c}{ U } & \multicolumn{1}{c}{ CV } & \multicolumn{1}{c}{ S } & \multicolumn{1}{c}{ H } & \multicolumn{1}{c}{ G } \\
trans. & 0.889 & 2.981 & 42.954 & 1.481 & -11.325 \\
rot. & 0.889 & 2.981 & 34.195 & 0.889 & -9.307 \\
vib. & 11.202 & 72.863 & 73.238 & 11.202 & -10.634 \\
elec. & 0.000 & 0.000 & 0.000 & 0.000 & 0.000 \\
total & 12.980 & 78.825 & 150.387 & 13.572 & -31.266
\end{tabular}

Total internal energy, Utot (SCFE + ZPE + U): -1282.274227 hartrees Total enthalpy, Htot (Utot + pV): -1282.273283 hartrees Total Gibbs free energy, Gtot (Htot - $\left.\mathrm{T}^{\star} \mathrm{S}\right)$ : $\quad-1282.344737$ hartrees

\section{Single point calculation at the B3LYP/LACV3P**++//B3LYP/LACVP** level}

Energy components, in hartrees:

(A) Nuclear repulsion......... 1816.08113663859

(E) Total one-electron terms .... -5362.72540077033

(I) Total two-electron terms .... 2263.75707415624

(J) Coulomb.............. 2412.94496348087

(K) Exchange+Correlation...... -149.18788932463

(L) Electronic energy.......... $-3098.96832661409 \quad(\mathrm{E}+\mathrm{I})$

(N) Total energy...........

SCFE: SCF energy: DFT(b3lyp) $\quad-1282.88718997550$ hartrees

HOMO energy: -0.25264

LUMO energy: -0.03333

B3LYP/LACV3P**++//B3LYP/LACVP** Total Gibbs free energy, Gtot (Htot - T*S): -1282.609375 hartrees

\section{Product}<smiles>C=CC[C@@]1(O)CC(=C)c2ccccc2C1</smiles>

NIMAG $\odot$ 


\begin{tabular}{|c|c|c|c|}
\hline C1 & -0.8403597483 & - ๑ . 7488229813 & -2.9680196856 \\
\hline $\mathrm{C} 2$ & -0.6819229183 & $-\odot .4398606755$ & -1.6048718416 \\
\hline C3 & ๑. 6190735005 & $-\odot .4062388460$ & -1.0580557802 \\
\hline $\mathrm{C} 4$ & 1.7105699874 & $-\odot .7142061848$ & -1.8803299742 \\
\hline C5 & 1.5389184202 & -1.0302679143 & -3.2244834962 \\
\hline $\mathrm{C} 6$ & ๑. 2536756251 & -1.0416253145 & -3.7727868756 \\
\hline C7 & $\odot .8699466279$ & $-\odot .0225884872$ & ๑. 3896860625 \\
\hline C8 & -0.3821065302 & $-\odot .0256714092$ & 1. 2894922531 \\
\hline C9 & -1.5465485058 & $\odot .6336530778$ & $\odot .5195735547$ \\
\hline C10 & -1.8489868218 & $-\odot .1652757279$ & -0.7281961908 \\
\hline C11 & -3.0776427959 & $-\odot .6569496507$ & -0.9614955809 \\
\hline 012 & $-\odot .7280469944$ & -1.3665136532 & 1.6524899623 \\
\hline C13 & $-\odot .0760865982$ & ๑. 6995928344 & 2.6176106305 \\
\hline C14 & -1.2195775637 & ๑. 6980345873 & 3.5938625826 \\
\hline C15 & -1.7484586378 & 1.7871716791 & 4.1536795488 \\
\hline H16 & 2.7108336970 & $-\odot .7026050176$ & -1.4531701718 \\
\hline $\mathrm{H} 17$ & 2.4012216744 & -1.2606699453 & -3.8438561004 \\
\hline H18 & ๑. 1084030810 & -1.2705848994 & -4.8246914149 \\
\hline H19 & -1.8358095648 & $-\odot .7358877989$ & -3.4016328871 \\
\hline $\mathrm{H} 20$ & 1.6104030096 & -0.6988084075 & $\odot .8322187317$ \\
\hline H21 & 1.3167820773 & ๑. 9820182661 & ๑. 407००58324 \\
\hline $\mathrm{H} 22$ & -2.4280676800 & $\odot .7020202275$ & 1.1624977523 \\
\hline H23 & -1.2515849014 & 1.6568105713 & ๑. 2494911817 \\
\hline $\mathrm{H} 24$ & ๑. 7842960558 & ๑. 1832892403 & 3.0676436506 \\
\hline $\mathrm{H} 25$ & ๑. 2393471729 & 1.7276046941 & 2.4044949926 \\
\hline H26 & -1.6189111636 & -0.2846127146 & 3.8374646055 \\
\hline H27 & -2.5680318834 & 1.7223694188 & 4.8637115109 \\
\hline $\mathrm{H} 28$ & -1.3776438902 & 2.7853658314 & 3.9301281306 \\
\hline $\mathrm{H} 29$ & $-1.232260 \odot \odot 29$ & -1.7538359481 & ๑. 9225008219 \\
\hline H30 & -3.3012056934 & -1.2811504932 & -1.8210570605 \\
\hline H31 & -3.9034709134 & $-\odot .4396257713$ & -0.2909681415 \\
\hline
\end{tabular}

Energy components, in hartrees:
(A) Nuclear repulsion..........
962.13421131045
(E) Total one-electron terms.....
$-2746.03815659178$
1165.57667038501
(I) Total two-electron terms.....
1256.25362508331
(J) Coulomb...............
$-90.67695469830$
(K) Exchange+Correlation......
$-1580.46148620677$
(L) Electronic energy..........
$-618.32727489632$

SCFE: SCF energy: DFT(b3lyp)

-618.32727489632 hartrees

HOMO energy: -0.22620

LUMO energy: $-\odot .03091$

Thermodynamic properties calculated assuming an ideal gas

In the table below, the units for temperature are kelvins, the units for $U, H$, and $G$ are $\mathrm{kcal} / \mathrm{mol}$ and the units for $\mathrm{Cv}$ and $\mathrm{S}$ are $\mathrm{cal} /(\mathrm{mol} \mathrm{K})$

The zero point energy (ZPE): $165.589 \mathrm{kcal} / \mathrm{mol}$ is not included in $U, H$, or $G$ in the table below

$\mathrm{T}=298.15 \mathrm{~K}$

\begin{tabular}{rrrrrr} 
& \multicolumn{1}{c}{ U } & \multicolumn{1}{c}{ CV } & \multicolumn{1}{c}{ S } & \multicolumn{1}{c}{ H } & \multicolumn{1}{c}{ G } \\
trans. & 0.889 & 2.981 & 41.786 & 1.481 & -10.977 \\
rot. & 0.889 & 2.981 & 31.967 & 0.889 & -8.642 \\
vib. & 6.763 & 48.611 & 40.798 & 6.763 & -5.401 \\
elec. & $0.00 \odot$ & $0.00 \odot$ & $0.00 \odot$ & $0.0 \odot \odot$ & $0.0 \odot \odot$ \\
total & 8.540 & 54.573 & 114.550 & 9.133 & -25.020
\end{tabular}

Total internal energy, Utot (SCFE + ZPE + U): -618.049783 hartrees Total enthalpy, Htot (Utot $+p V)$ : -618.048839 hartrees Total Gibbs free energy, Gtot (Htot - $T^{*} S$ ): -618.103265 hartrees 


\section{Single point calculation at the B3LYP/LACV3P**++//B3LYP/LACVP** level}

Energy components, in hartrees:

(A) Nuclear repulsion...........

(E) Total one-electron terms.....

(I) Total two-electron terms.....

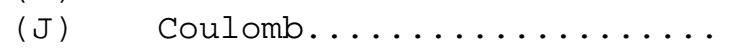

(K) Exchange+Correlation.......

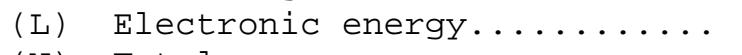

(N) Total energy.............
962.13421131379

$-2745.52076924442$

1164.90246605398

1255.57397923086

$-90.67151317688$

$-1580.61830319044 \quad(E+I)$

$-618.48409187664 \quad(A+L)$

SCFE: SCF energy: DFT(b3lyp) -618.48409187664 hartrees

HOMO energy: $\quad-0.23854$

LUMO energy: $\quad-0.04906$

B3LYP/LACV3P**++//B3LYP/LACVP** Total Gibbs free energy, Gtot (Htot - $T^{*} S$ ): -618.260082 hartrees

\section{HOMs}

NIMAG $\odot$

$\begin{array}{lrrr}\text { H1 } & 0.8291501375 & -0.1930598803 & -1.9509198420 \\ 02 & 0.0635675884 & -0.7437705789 & -1.7131968669 \\ \text { S3 } & -1.2379537152 & 0.2660019546 & -1.6929444310 \\ \text { C4 } & -2.0290531259 & -0.3714908378 & -0.2175207492 \\ 05 & -2.0821515092 & -0.0198540410 & -2.8442505856 \\ \text { 06 } & -0.7261772652 & 1.6149602409 & -1.4546447900 \\ \text { H7 } & -1.3727597920 & -0.2029994318 & 0.6358230888 \\ \text { H8 } & -2.9649602561 & -.1772004261 & -0.1011168351 \\ \text { H9 } & -2.2277029975 & -1.4338759854 & -0.3611772144\end{array}$

Energy components, in hartrees:
(A) Nuclear repulsion..........
277.05468552549
(E) Total one-electron terms..... $-1463.63018588445$
(I) Total two-electron terms.....
522.26632853591

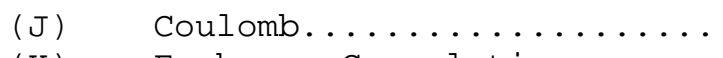
580.77385144174
(K) Exchange+Correlation......
$-58.50752290583$
(L) Electronic energy..........
$-941.36385734854$
(N) Total energy.............
$-664.30917182305$
$(\mathrm{E}+\mathrm{I})$
$(A+L)$

SCFE: SCF energy: DFT

-664.30917182305 hartrees

HOMO energy: $\quad-0.30593$

LUMO energy: $\quad 0.03161$

Thermodynamic properties calculated assuming an ideal gas

In the table below, the units for temperature are kelvins, the units for $U, H$, and $G$ are $\mathrm{kcal} / \mathrm{mol}$ and the units for $\mathrm{Cv}$ and $\mathrm{S}$ are $\mathrm{cal} /(\mathrm{mol} \mathrm{K})$

The zero point energy (ZPE): $\quad 38.690 \mathrm{kcal} / \mathrm{mol}$ is not included in $U, H$, or $G$ in the table below 


\begin{tabular}{|c|c|c|c|c|c|}
\hline & U & CV & $\mathrm{S}$ & $\mathrm{H}$ & $G$ \\
\hline & ------- & ------- & -------- & ------- & ------- \\
\hline trans. & 0.889 & 2.981 & 39.596 & 1.481 & -10.324 \\
\hline rot. & 0.889 & 2.981 & 25.691 & 0.889 & -6.771 \\
\hline vib. & 1.993 & 14.191 & 11.162 & 1.993 & -1.335 \\
\hline elec. & 0.000 & $\odot .00 \odot$ & $\odot .0 \odot \odot$ & 0.000 & $\odot . \odot \odot \odot$ \\
\hline total & 3.770 & 20.152 & 76.449 & 4.363 & $-18 \cdot 431$ \\
\hline
\end{tabular}

Total internal energy, Utot (SCFE + ZPE + U): $\quad-664.241508$ hartrees Total enthalpy, Htot (Utot + pV): -664.240563 hartrees Total Gibbs free energy, Gtot (Htot - $T^{\star} S$ ): $\quad-664.276887$ hartrees

\section{Single point calculation at the B3LYP/LACV3P**++//B3LYP/LACVP** level}

Energy components, in hartrees:
(A) Nuclear repulsion.......... 277.06128496740
(E) Total one-electron terms.... -1463.45973972565
(I) Total two-electron terms.... 521.97418187429
(J) Coulomb.............. 580.49526630838
(K) Exchange+Correlation...... $\quad-58.52108443409$
(L) Electronic energy.......... -941.48555785136 (E+I)
(N) Total energy ........... -664.42427288396 (A+L)

SCFE: SCF energy: DFT $\quad-664.42427288396$ hartrees

HOMO energy: $\quad-0.32349$

LUMO energy: $\quad-0.02880$

B3LYP/LACV3P**++//B3LYP/LACVP** Total Gibbs free energy, Gtot (Htot - $T^{*} S$ ): -664.391988 hartrees 